University of Louisville

ThinkIR: The University of Louisville's Institutional Repository

Electronic Theses and Dissertations

$12-2010$

\title{
Nanowire based materials and architectures as anodes for LI-ION batteries.
}

Praveen Meduri 1982-

University of Louisville

Follow this and additional works at: https://ir.library.louisville.edu/etd

\section{Recommended Citation}

Meduri, Praveen 1982-, "Nanowire based materials and architectures as anodes for LI-ION batteries."

(2010). Electronic Theses and Dissertations. Paper 959.

https://doi.org/10.18297/etd/959

This Doctoral Dissertation is brought to you for free and open access by ThinkIR: The University of Louisville's Institutional Repository. It has been accepted for inclusion in Electronic Theses and Dissertations by an authorized administrator of ThinkIR: The University of Louisville's Institutional Repository. This title appears here courtesy of the author, who has retained all other copyrights. For more information, please contact thinkir@louisville.edu. 


\title{
NANOWIRE BASED MATERIALS AND ARCHITECTURES AS ANODES FOR LI- ION BATTERIES
}

\author{
By \\ Praveen Meduri \\ Master of Science, University of Akron, 2005
}

\author{
A Dissertation \\ Submitted to the Faculty of the \\ Graduate School of the University of Louisville \\ In Partial Fulfillment of the Requirements \\ for the Degree of \\ Doctor of Philosophy \\ Department of Chemical Engineering \\ University of Louisville \\ Louisville, KY 40292
}

December 2010 


\title{
NANOWIRE BASED MATERIALS AND ARCHITECTURES AS ANODES FOR LI-
} ION BATTERIES

\author{
By \\ Praveen Meduri \\ Master of Science, University of Akron, 2005
}

A Dissertation Approved on

October 26, 2010

By the Following Dissertation Committee

Dr. Mahendra K. Sunkara (Dissertation Director)

Dr. Thomas L. Starr

Dr. Gamini U. Sumanasekera

Dr. Moises A. Carreon

Dr. Frank P. Zamborini 


\section{DEDICATION}

This dissertation is dedicated to my parents

Brahmeswara Rao Meduri and Vijaya Meduri, sister Anusha Meduri, beloved wife Prasanthi and all my dear friends. 


\section{ACKNOWLEDGEMENTS}

First of all, I am thankful to Dr. Mahendra K Sunkara for giving me an opportunity to be a part of the chemical vapor deposition group. I am also grateful to him for introducing me to the exciting areas of nanomaterials and energy storage, which are bound to overcome the present environmental challenges. In particular, I appreciate his constant motivation, guidance and feedback. I have always cherished the independence he gave me in working on the project of utmost interest and guiding though the major hurdles of the project.

My special thanks to Dr. Gamini Sumanasekera, Dr. Thomas Starr, Dr. Moises Carreon and Dr. Frank Zamborini for serving as my committee members. I appreciate their feedback and suggestions which led to interesting scientific studies.

I would like to thank all my lab members, both present and past for helping me with my work and making my stay enjoyable. I would also like to thank the Department of Chemical Engineering, University of Louisville for giving me this opportunity.

Last but certainly not the least; I would like to thank my family and my wife for their love and encouragement all throughout my life.

Praveen Meduri

October 2010 


\section{ABSTRACT \\ NANOWIRE BASED MATERIALS AND ARCHITECTURES AS ANODES FOR LI- ION BATTERIES \\ Praveen Meduri \\ October $26^{\text {th }} 2010$}

Energy independence requires that the nation reduce its dependence on foreign oil imports. This can be achieved through electrification of transportation vehicles if proper battery technology can be developed. In addition, the renewable energy sources such as solar and wind tend to be intermittent with time scales ranging from seconds to hours. So, a suitable energy storage technology is essential for integrating renewable sources for base load generation. Lithium ion battery technology is promising; however, the big challenge limiting its widespread implementation is with capacity, durability and safety. A dramatic advancement is needed in terms of materials used for both electrodes and electrolytes.

Several materials such as tin, tin oxide $\left(\mathrm{SnO}_{2}\right)$, cobalt oxide $\left(\mathrm{CoO}_{3}\right)$, iron oxide $\left(\mathrm{Fe}_{2} \mathrm{O}_{3}\right)$, intermetallic alloys and semiconductors like silicon $(\mathrm{Si})$ and germanium $(\mathrm{Ge})$ potentially provide much higher theoretical capacity compared to conventionally used carbon based materials for anodes. Although most of these materials have favorable characteristics, they come at the expense of enormous volume changes associated with lithium alloying as a result of which the material integrity is lost. One-dimensional 
nanowires are believed to have better charge transport and strain relaxation properties but mostly unproven.

In this dissertation, a generic hybrid architecture concept involving onedimensional nanowires covered with nanoclusters is proposed for improving the durability of anodes with high capacity retention. Specifically, this concept is demonstrated with metal-nanocluster-covered metal oxide nanowires using $\mathrm{Sn} / \mathrm{SnO}_{2}$ system. The results showed that $\mathrm{Sn}$ nanocluster covered $\mathrm{SnO}_{2}$ nanowires exhibited a capacity retention of $\sim 800 \mathrm{mAhg}^{-1}$ for up to 100 cycles, the highest reported until now. In this study, the presence of well-spaced nanoclusters provides adequate room for metal volume expansion on lithiation preventing cluster coalescence leading to stable material structure while the metal oxide base provides various channels for electron conductivity. Cyclic voltammetric studies are conducted to understand the fundamental behavior of monolayers of nanoscale and micron scale tin clusters supported on both metallic substrates and hybrid architectures. The results suggest that tin clusters with sizes less than 50nm undergo complete de-lithiation while larger clusters exhibit incomplete delithiation due to diffusion limitation.

The hybrid architecture concept can also be extended to other high capacity materials systems using unique carbon structures and molybdenum oxide nanowire arrays as base materials. In this direction, carbon microtubes (CMTs) are synthesized in large quantities and tested for their lithiation and de-lithiation characteristics. CMTs are micron sized tubes with $50 \mathrm{~nm}$ walls comprised of random nanographite domains. The results indicated that CMTs exhibited capacity retention of $\sim 440 \mathrm{mAhg}^{-1}$, higher than the theoretical capacity of graphite. More importantly, CMTs show excellent rate capability 
of $\sim 135 \mathrm{mAhg}^{-1}$ at rates as high as $5 \mathrm{C}$ which makes them ideal as base materials in hybrid architectures. Another material of interest is molybdenum oxide $\left(\mathrm{MoO}_{3}\right)$ which has excellent theoretical capacity and stability. Nanowire arrays are grown on conducting substrates providing direct charge conduction pathways eliminating the use of conducting polymer, generally used in powder based electrodes. These arrays show good capacity retention of $\sim 630 \mathrm{mAhg}^{-1}$ along with rate capability. In addition, the capacity retention below $0.7 \mathrm{~V}$ is $\sim 500 \mathrm{mAhg}^{-1}$, which is better than the performance of any other $\mathrm{MoO}_{3}$ based materials and hence, makes the material viable for practical application as electrodes.

Technologically, the proposed concept of hybrid architectured materials involving 1-D materials with nanoclusters should result in the development of new materials architectures for high capacity, high rate and durable anodes. Scientifically, for the first time, the study showed fundamental differences in the lithiation/de-lithiation behavior of tin clusters at nanoscale which could apply to several other material systems. In addition, the interesting aspects involved in high capacity retention and durability have been aptly studied and understood for further application in other material systems. 


\section{TABLE OF CONTENTS}

PAGE

ACKNOWLEDGEMENTS ......................................................

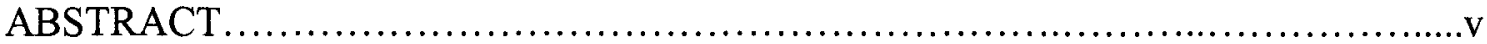

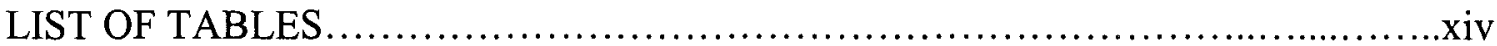

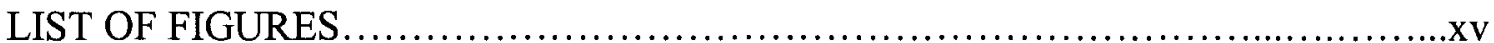

\section{CHAPTER}

1. Introduction

1.1. Global energy challenges......................................................

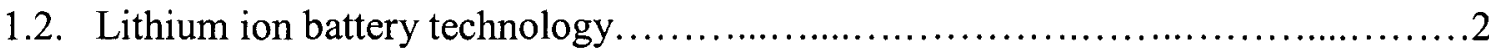

1.2.1. LIBs: Mechanism of lithium ion battery operation...........................

1.2.2. Basic principles of operation in LIBs....................................

1.2.3. Electrode materials for LIBs........................................

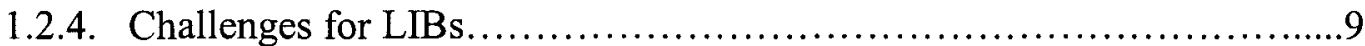

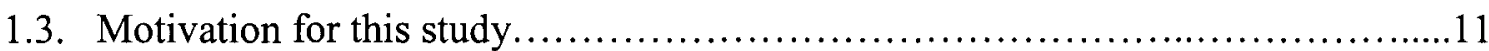

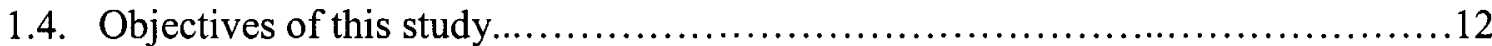

1.5. Dissertation outline............................................................. 16 
2. Background

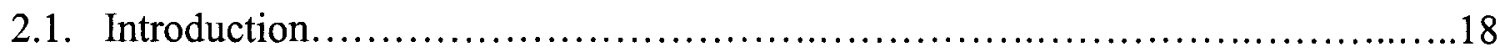

2.2. Electrochemical energy storage systems............................... 18

2.2.1. History of storage systems...................................20

2.2.2. Overview of different battery system characteristics...................21

2.3. Carbon based materials as anodes in LIBs.....................................23

2.3.1. Graphitic carbons as anodes for LIBs...........................23

2.3.1.1. Multi walled carbon nanotubes...............................24

2.3.1.2. Single walled carbon nanotube...............................25

2.3.1.3. Carbon nanofibers.......................................26

2.3.1.4. Other carbon based materials...............................27

2.3.2. Non-graphitic carbons as anodes for LIBs.........................29

2.4. Metal oxide electrodes as anode materials.................................. 31

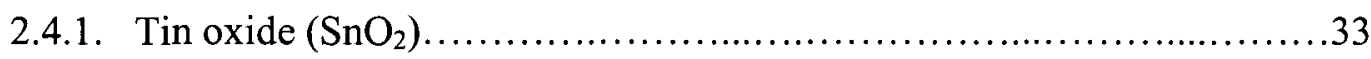

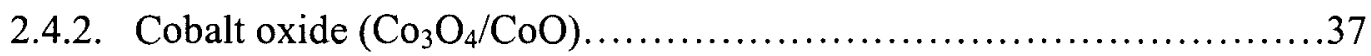

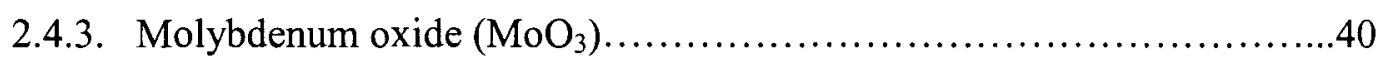

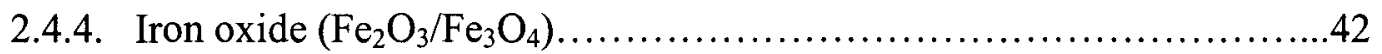

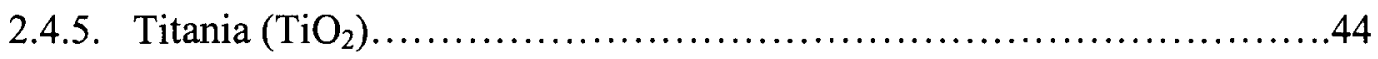

2.4.6. Other metal oxides as anodes for LIBs...........................45

2.5. Inorganic material anodes............................................ 48

2.5.1. Silicon nanomaterials........................................... 48

2.5.2. Germanium nanomaterials.......................................50

2.5.3. Alloy composites..............................................51 
2.6. Three-dimensional nanostructured materials................................52

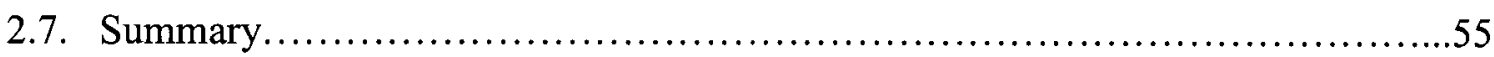

3. Experimental Techniques

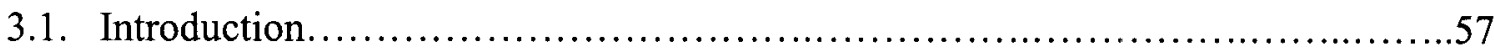

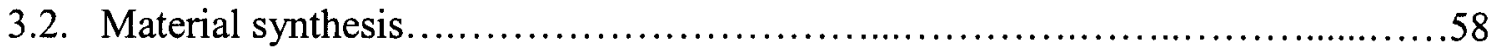

3.2.1. $\mathrm{SnO}_{2}$ nanowires...............................................58

3.2.1.1. $\mathrm{SnO}_{2}$ nanowires on substrates................................58

3.2.1.2. Gas phase production of $\mathrm{SnO}_{2}$ nanowire powders.................59

3.2.2. $\mathrm{MoO}_{3}$ and $\mathrm{WO}_{3}$ nanowire arrays..............................61

3.2.3. Carbon microtubes.............................................. 63

3.2.4. Hybrid architectured nanowires...................................64

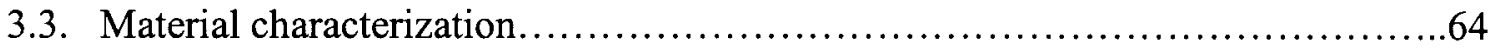

3.4. Lithium ion battery electrodes......................................... 65

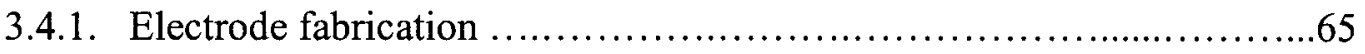

3.4.2. Experimental setup...........................................66

3.4.3. Electrochemical testing.....................................67

3.4.3.1. Constant current galvanostatic measurements...................67

3.4.3.2. Differential capacity curves................................68

3.4.3.3. Cyclic voltammetry........................................69

3.4.3.4. Post-lithiation analysis................................... 72

4. Hybrid architectured nanowires as lithium ion battery anodes

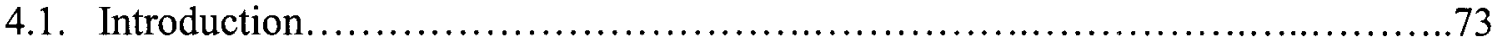

4.2. One-dimensional tin oxide nanowires as anodes in LIBs .......................74 
4.2.1. Large scale synthesis............................................... 74

4.2.2. Structural properties..............................................76

4.2.3. Electrochemical properties....................................... 78

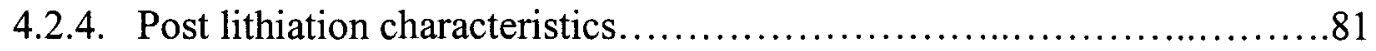

4.3. Metal nanocluster covered metal oxide nanowires (hybrid architectures) as high

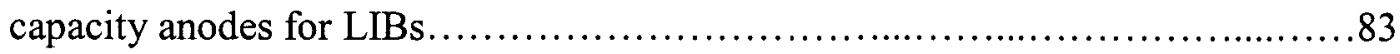

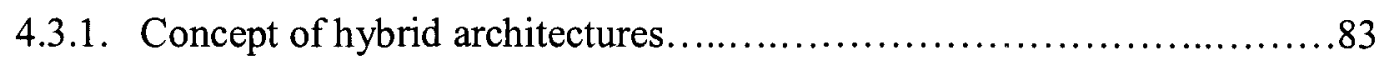

4.3.2. Synthesis and physical characteristics of $\mathrm{Sn}$ nanocluster covered $\mathrm{SnO}_{2}$

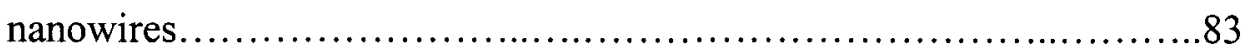

4.3.3. Enhanced electrochemical properties.............................86

4.3.4. Post lithiation characterization.......................................90

4.4. Mixed metal-metal oxide materials as anodes for LIBs.........................99

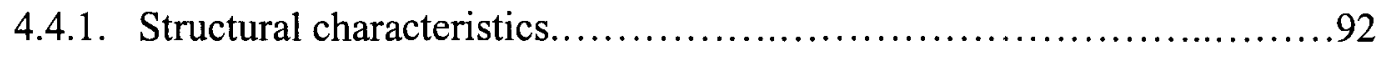

4.4.2. Electrochemical characteristics...................................93

4.5. Specific capacity performance of different $\mathrm{Sn} / \mathrm{SnO}_{2}$ architectures................95

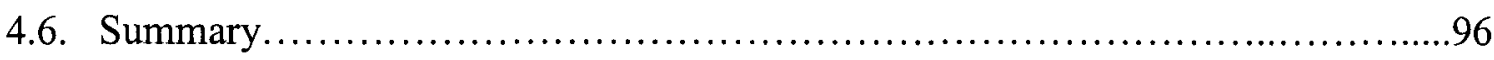

5. Lithiation/de-lithiation kinetics of hybrid architectured nanowires

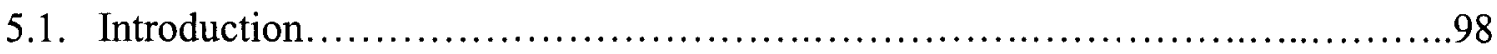

5.2. High capacity retention of hybrid architectures and capacity degradation of other

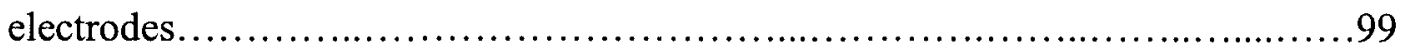

5.2.1. Mechanism of lithiation/de-lithiation of hybrid materials..................99

5.2.2. Comparison of lithiation/de-lithiation mechanisms of different

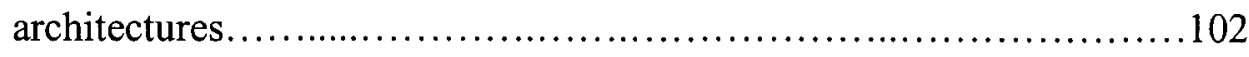


5.2.3. Lithiation/de-lithiation characteristics as a function of particle

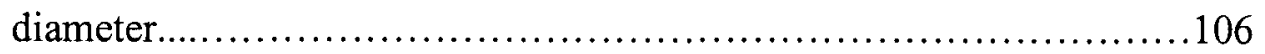

5.2.4. Li concentration profile analysis...................................... 109

5.3. Rate capability of hybrid architectured nanowires.................................113

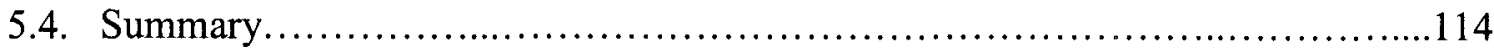

6. Thin walled carbon microtubes as high rate base material for hybrid architecture anodes

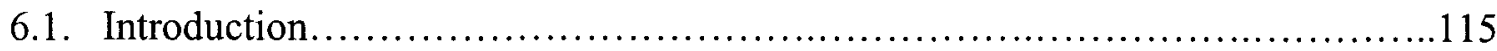

6.2. One-dimensional carbon based materials ....................................116

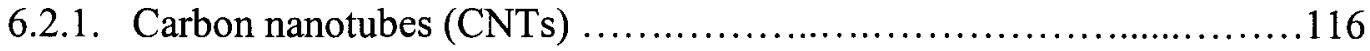

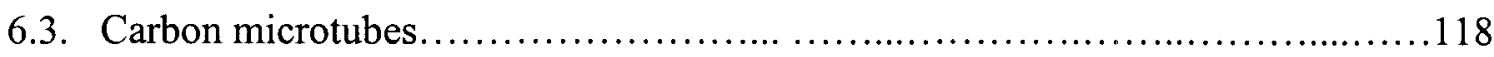

6.3.1. Large area synthesis of CMTs ....................................... 118

6.3.2. Physical characteristics of thin walled CMTs........................119

6.3.3. Superior electrochemical characteristics of CMTs.....................125

6.3.3.1. High capacity performance ................................... 125

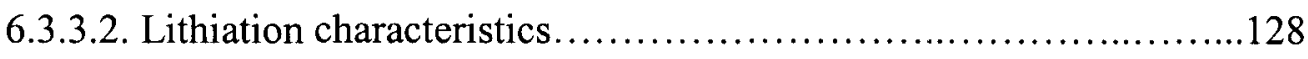

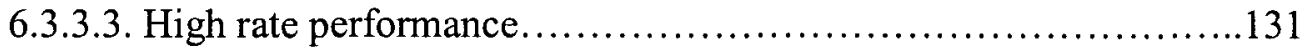

6.3.4. Lithiation mechanism in CMTs......................................132

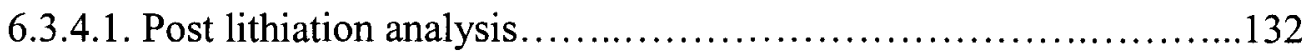

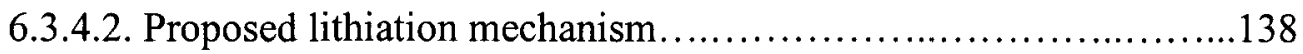

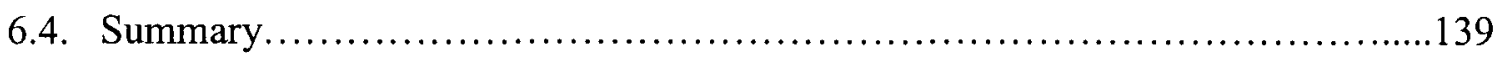

7. Nanowire arrays as anodes and base materials for hybrid architectures

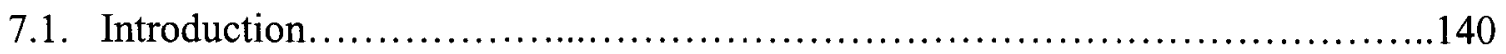


7.2. Synthesis of nanowire arrays directly on conducting substrates.

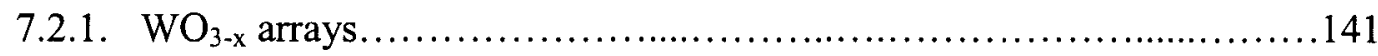

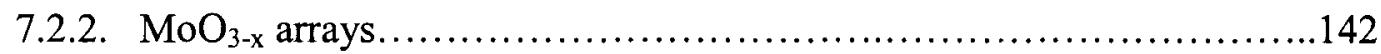

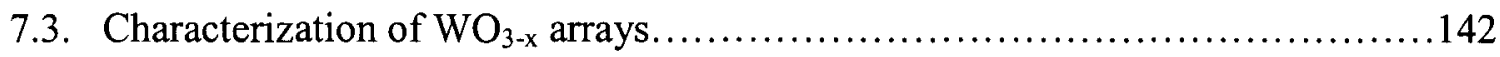

7.3.1. Physical characteristics........................................ 142

7.3.2. Electrochemical characteristics.................................... 145

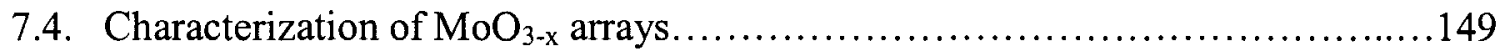

7.4.1. Physical properties................................................... 149

7.4.2. Electrochemical properties.................................... 151

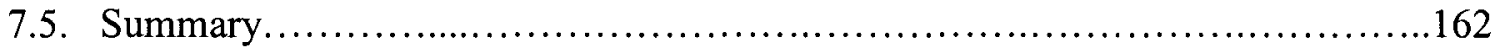

8. Conclusions............................................................ 163

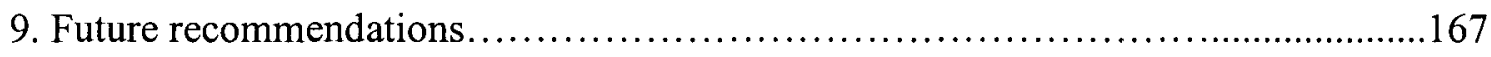

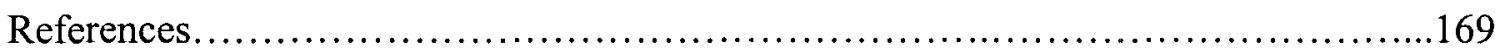

Curriculum vitae.........................................................191 


\section{LIST OF TABLES}

TABLE

PAGE

1.1. Theoretical specific capacities of different materials.............................8

2.1. Comparison of various energy storage technologies..........................19

2.2. Performance characteristics of different battery types..............................22

2.3. Summary of retained capacities of metal oxide nanomaterials..................46

6.1. Rate performance of carbon microtubes compared with other carbonaceous

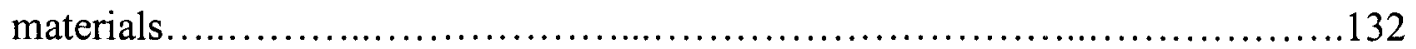




\section{LIST OF FIGURES}

FIGURE

PAGE

1.1. Charge-discharge mechanisms in LIBs...........................................3

1.2. Electrolyte window with electrode electrochemical potentials.....................5

1.3. Electrode material characteristics of both anodes and cathodes.......................7

1.4. Schematic showing the strain, volume accommodation in $1 \mathrm{D}$ nanowires whereas the thin films and nanoparticles undergo mechanical fracture........................14

2.1. Energy density of different battery systems ....................................

3.1. Schematic of the CVD reactor used for $\mathrm{SnO}_{2}$ nanowire synthesis...................59

3.2. Schematic of downstream plasma reactor used for large scale synthesis of $\mathrm{SnO}_{2}$

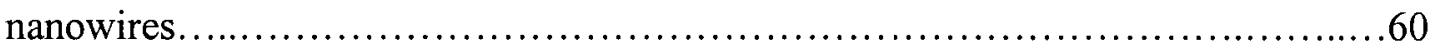

3.3. Schematic of the HFCVD system for the metal oxide nanowire array synthesis.....62

3.4. Schematic of MOCVD reactor indicating precursor delivery system along with the

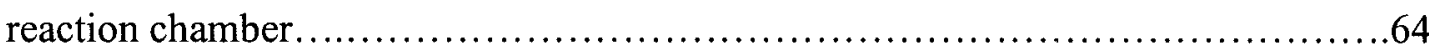

3.5. Schematic of the flooded three cell configuration.................................66

3.6. Photograph of the Vacuum Atmospheres Company glove box....................66

3.7. Typical curve used in determining the specific capacity ........................68

3.8. Cyclic voltammetry curve indicating the lithiation and de-lithiation peaks..........71

4.1. SEM images of the as synthesized $\mathrm{SnO}_{2}$ nanowires grown directly on quartz at

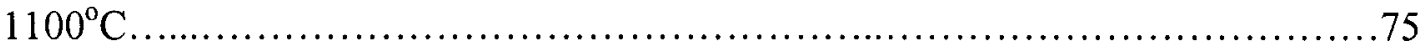


4.2. XRD spectra of pure phase $\mathrm{SnO}_{2}$ nanowires indicating their crystalline nature.....76

4.3. Raman spectrum of pure phase $\mathrm{SnO}_{2}$ nanowires............................77

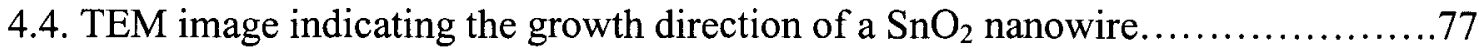

4.5. Specific capacity retention of pure $\mathrm{SnO}_{2}$ nanowires with cycling for up to 40

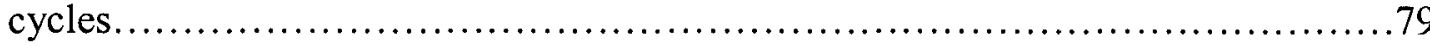

4.6. Cyclic voltammetry of $\mathrm{SnO}_{2}$ nanowires for the first 5 cycles with peak positions at a

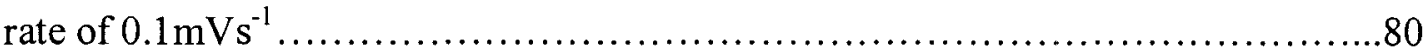

4.7. XRD comparison of pre and post lithiated $\mathrm{SnO}_{2}$ nanowires...................

4.8. $\mathrm{SEM}$ images of $\mathrm{SnO}_{2}$ nanowires after lithiation showing the crumbled nanowires. 82

4.9. Schematic of metal-nanocluster-covered metal oxide nanowire with adequate spacing to accommodate volume expansion.

4.10. SEM images of $\mathrm{Sn}$-nanocluster-covered $\mathrm{SnO}_{2}$ nanowires with $\mathrm{Sn}$ nanoclusters clearly visible on the surface.

4.11. TEM images of hybrid architectures with $15 \mathrm{~nm} \mathrm{Sn}$ nanoclusters on the nanowire periphery .85

4.12. XRD spectrum of hybrid architectures clearly indicating the presence of $\mathrm{Sn}$ after $\mathrm{H}_{2}$ reduction .86

4.13. Specific capacity (in circles) and columbic efficiency (triangles) of Sn-nanoclustercovered $\mathrm{SnO}_{2}$ nanowires until 100 cycles indicating excellent cycling behavior...87

4.14. The voltage-capacity curves of the hybrid structures for first eight cycles between 0.01 and $2.2 \mathrm{~V}$ performed at a rate of $100 \mathrm{mAg}^{-1}$ and at room temperature .88

4.15. Cyclic voltammetry of hybrid nanowires for the first 5 cycles with peak positions at a rate of $0.1 \mathrm{mVs}^{-1}$ 
4.16. Hybrid architecture nanowires after charge-discharge cycles: a) SEM image. b) TEM image showing well spaced $\mathrm{Sn}$ nanoclusters

4.17. XRD spectrum of the hybrid nanowires after 100 charge-discharge cycles indicating the presence of $\mathrm{SnO}$ and $\mathrm{Sn}$.

4.18. $\mathrm{SEM}$ images of mixed $\mathrm{Sn} / \mathrm{SnO}_{2}$ nanowires

4.19. Specific capacity retention of mixed $\mathrm{Sn} / \mathrm{SnO}_{2}$ nanowires with cycling for up to 40

cycles

4.20. Specific capacity retention of various mixed $\mathrm{Sn} / \mathrm{SnO}_{2}$ architectures with cycling until 40 cycles.

5.1.XRD spectra of both pure phase $\mathrm{SnO}_{2}$ nanowires and hybrid architectures after lithiation.

5.2. (a) Brightfield TEM image of hybrid nanowire that was subjected to hundred chargedischarge cycles. (b) High resolution image of the nanowire shown in (a). The small domains correspond to $\mathrm{SnO}$ phase.

5.3. Schematic illustration of lithiation and de-lithiation processes in Sn-nanoclustercovered $\mathrm{SnO}_{2}$ nanowires

5.4. Cyclic voltammetry curves of pure phase $\mathrm{SnO}_{2}$ nanowires for the first 5 cycles with peak positions. Scan rate: $0.1 \mathrm{mVs}^{-1}$

5.5. Cyclic voltmmetry curves of $\mathrm{Sn}$ nanocluster covered $\mathrm{SnO}_{2}$ nanowires for the first 5 cycles with peak positions. Scan rate: $0.1 \mathrm{mVs}^{-1}$

5.6. SEM images of (a) Micron scale Sn clusters/thin films synthesized directly on conducting substrates. (b) Nanoscale Sn crystals with an average diameter of $\sim 50$ $\mathrm{nm}$. 
5.7. Cyclic voltammetry curves of micron scale Sn/thin films for the first 5 cycles with peak positions. Scan rate: $0.1 \mathrm{mVs}^{-1}$

5.8. Cyclic voltammetry curves of $\mathrm{Sn}$ nanoclusters grown on conducting substrates for the first 5 cycles with peak positions. Scan rate: $0.1 \mathrm{mVs}^{-1}$

5.9. Cyclic voltammetry profiles on Sn clusters grown on conducting substrates at various scan rates.

5.10. Plots of (a) scan rate vs. peak potential at $0.61 \mathrm{~V}$ peak during the first cycle. (b) scan rate ${ }^{0.5}$ vs. peak current at $0.61 \mathrm{~V}$ peak (first cycle).

5.11. De-lithiation concentration profiles of micron sized and nanosized particles with changing time constants. The figure clearly indicates a vast difference in the profiles showing single phase de-lithiation in micron sized particles and stage-wise delithiation in nanoparticles.

5.12. Charge-discharge cycling performance of $\mathrm{Sn}$-nanocluster-covered $\mathrm{SnO}_{2}$ nanowires at various current densities

6.1. SEM image of MWCNTs with $100 \mathrm{~nm}$ diameters and several microns length......117

6.2. Discharge performance of pure MWCNTs at a current density of $25 \mathrm{mAhg}^{-1} \ldots \ldots 118$

6.3. Representative images of large areas of bundled carbon microtubes as seen in SEM

6.4. TEM image of multiple CMTs devoid of gallium

6.5. EDX spectrum of the cleaned CMTs showing the only element carbon (significantly low traces of gallium) 
6.6. TEM images showing (a) CMT diameter and wall thickess of $\sim 50 \mathrm{~nm}$. (b) CMT wall domains in a HRTEM. Inset shows the structure of a single graphite nanodomain.)

6.7. Characterization of cleaned CMTs using Raman spectroscopy for the bonding state of the carbon atoms in CMTs.

6.8. Characterization of cleaned CMTs using X-ray diffraction spectrum showing the material crystal structure and phase.

6.9. Cyclic performance at a current density of $25 \mathrm{mAhg}^{-1}$. Columbic efficiency is presented on the secondary axis to the right.

6.10. Electrochemical characteristics of CMTs with charge-discharge curves indicating minimal capacity loss after 3 cycles.

6.11. Differential capacity curves of the first 5 cycles of the CMTs cycled at a rate of 25 $\mathrm{mAhg}^{-1}$ between $3 \mathrm{~V}$ and $40 \mathrm{mV}$ 128

6.12. Cyclic voltammetry of CMTs indicating different peaks during the charge-discharge cycles for the first 8 cycles

6.13. CMTs performance at different charge and discharge rates.

6.14. Post-lithiation analysis using Raman spectroscopy after 20 charge-discharge cycles for CMTs.

6.15. Phase and structural changes in the material characterized by XRD after 20 cycles .134

6.16. SEM image showing the physical structure of the CMTs 135

6.17. TEM images of CMTs $(a, b, c, d)$ Pure CMTs devoid of gallium after acid treatment prior to cycling measurements. (e, f, g, h) CMTs after 20 cycles of lithiation and de- 
lithiation showing the presence of SEI layer but the graphite nanodomains are still clearly evident on the surface which are the pathways for $\mathrm{Li}$-ion diffusion........137

7.1. SEM images of as synthesized $\mathrm{W}_{18} \mathrm{O}_{49}$ nanowires. (a) Top view (b) Side view.....142

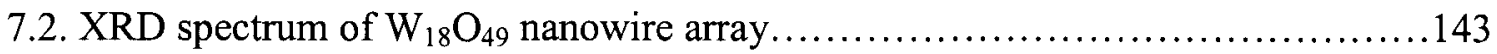

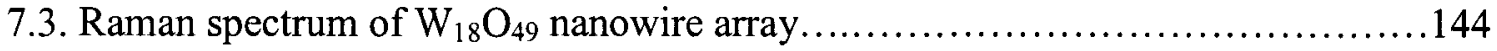

7.4. HRTEM image of an individual $\mathrm{W}_{18} \mathrm{O}_{49}$ nanowire showing high crystallinity all

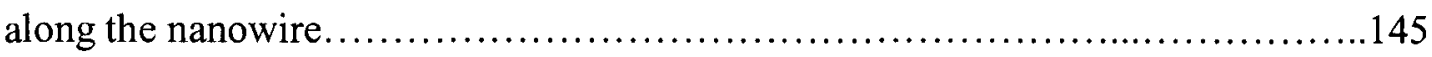

7.5. Specific capacity of $\mathrm{W}_{18} \mathrm{O}_{49}$ nanowires at a current density of $25 \mathrm{mAg}^{-1} \ldots \ldots \ldots \ldots 146$

7.6. First cycle charge-discharge curves of $\mathrm{W}_{18} \mathrm{O}_{49}$ nanowire arrays..................147

7.7. Charge-discharge cycles $2-5$ of the $\mathrm{W}_{18} \mathrm{O}_{49}$ nanowires..........................

7.8. Post-lithiated SEM images of nanowire arrays after 20 charge-discharge cycles...148

7.9. $\mathrm{SEM}$ images of as synthesized $\mathrm{Mo}_{17} \mathrm{O}_{47}$ nanowires............................149

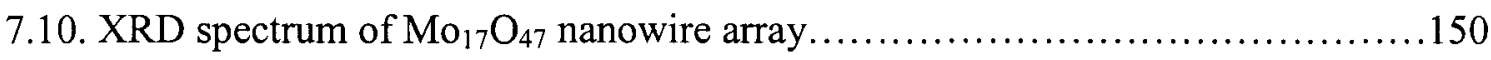

7.11. Raman spectrum of $\mathrm{Mo}_{17} \mathrm{O}_{47}$ nanowire arrays with multiple peak positions.....151

7.12. Cycling performance of $\mathrm{MO}_{3-\mathrm{x}}$ nanowire arrays. The secondary axis indicates the columbic efficiency

7.13. Discharge voltage profiles of $\mathrm{MO}_{3-\mathrm{x}}$ arrays for up to 10 cycles. The discharge voltage profiles are nearly same after the $3^{\text {rd }}$ cycle...........................154

7.14. Charge voltage profiles of $\mathrm{MO}_{3-\mathrm{x}}$ arrays for up to 10 cycles showing similar profiles for all the cycles.

7.15. CVs of $\mathrm{MO}_{3-\mathrm{x}}$ arrays for up to 5 cycles showing peaks pertaining to $\mathrm{Li}$ penetration 
7.16. DC curves of discharge cycles for the first 10 cycles indicating lithiation in 2 stages.............................................................. 156

7.17. DC curves of the charge cycles for the first 10 cycles indicating de-lithiation in 2 stages............................................................ 157

7.18. Specific capacity retention of $\mathrm{MoO}_{3-x}$ arrays in the potential range of $0.7-$

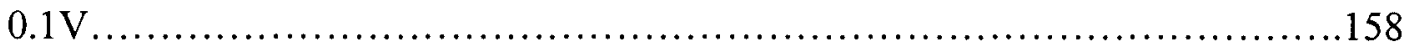

7.19. Rate performance of $\mathrm{MoO}_{3-\mathrm{x}}$ arrays indicating stable capacity retention. 159

7.20. Si $/ \mathrm{MoO}_{3-\mathrm{x}}$ hybrid architectures (a) Line scan in a TEM showing $10 \mathrm{~nm}$ silicon coating on $\mathrm{MoO}_{3-\mathrm{x}}$ nanowires. (b) Specific capacity of hybrid materials with capacity retentions of 780 and $~ 580 \mathrm{mAhg}^{-1}$ at rates of 100 and $200 \mathrm{mAg}^{-1}$, respectively 


\section{CHAPTER 1}

\section{INTRODUCTION}

\subsection{Global energy challenges}

The global demand for energy is continuously on the rise since the late $18^{\text {th }}$ century. The demand for energy has increased from 13.6 terawatts to 16.35 terawatts between 2000 and $2007^{1}$. It is projected to increase to $24.4 \mathrm{TW}$ in the year $2035^{1}$. In the present scenario, more than twice the energy is obtained from coal, oil and natural gas when compared to renewable energy sources. However, in 2035 the energy generated from renewable sources is almost doubled, whereas the other sources do not increase as much due to the dwindling oil and natural gas supplies and alleviated environmental concerns of global warming due to fossil fuel use ${ }^{1}$. This presents an ideal situation to focus efforts in developing and utilizing renewable energy sources to meet the increasing energy needs of the future. The various sources of renewable energy are solar, biomass, geothermal, hydrothermal and wind. The major sources of energy in the modern era are the biomass and hydrothermal even though the highest available reserve is solar, the energy of the future.

The renewable sources such as solar and wind are intermittent sources and thus can not be used for the base load. A suitable energy storage technology is necessary to integrate renewable energy sources in to our existing power grid. It can be ascertained here that energy storage also balances the supply and demand of energy. One third of US 
energy demand is for fuels in transportation sector. USA depends primarily from foreign sources for fuels which is a source of concern for both economic and national security reasons. In this regard, the electrification of transportation vehicles is of utmost interest from energy independence point of view. The electrification of transportation vehicles will also put more demand for electricity generation. Lithium ion battery technology is considered as the major technology for automotive applications.

\subsection{Lithium ion battery technology}

$\mathrm{Li}$ is the lightest metal with highest voltage and hence, is the main attraction for light weight batteries. Li-ion batteries have revolutionized the high-performance rechargeable-battery market due to their numerous attractive characteristics like lighter weight, higher voltage and store higher energy density (unit mass and unit volume), longer battery life, low self-discharge, and longer shelf life. Li-ion batteries are attractive for most of the portable power applications like cellular phones, laptops, medical devices. Recently, there is strong push for developing LIBs for widespread use in transportation applications like electric vehicles (EV), hybrid electric vehicles (HEV), plug-in hybrid electric vehicles (PHEV), heavy duty hybrids along with grid stabilization applications. The next section indicates the strong LIB presence throughout the globe. The present world market for LIBs is 10 billion dollars and continuously on the rise $\mathrm{e}^{2}$. However, their use in HEVs/PHEVs has been limited and their market estimation which is $\$ 2$ billion in 2009 is believed to raise as much as $\$ 5.2$ billion by $2015^{3}$. It is therefore necessary to develop long lasting, high performance LIBs as the future sources of energy. 


\subsubsection{LIBs: Mechanism of lithium ion battery operation}

A typical LIB consists of a transition metal oxide as the cathode and carbon as the anode. The commonly used cathodes are lithium cobalt oxide $\left(\mathrm{LiCoO}_{2}\right)$, lithium manganese oxide $\left(\mathrm{LiMn}_{2} \mathrm{O}_{4}\right)$ and variations of these with additional transition metals. The most widely used anode is graphite. The cell as assembled is in the discharged state. During the charging process, the $\mathrm{Li}$ ions pass through the electrolyte into the graphite host material and the electrons take the external circuit route. Cathode potential rises with $\mathrm{Li}$ ion removal and the anode potential decreases with $\mathrm{Li}$ ion uptake which gives rise to the voltage of the battery during the charging process.
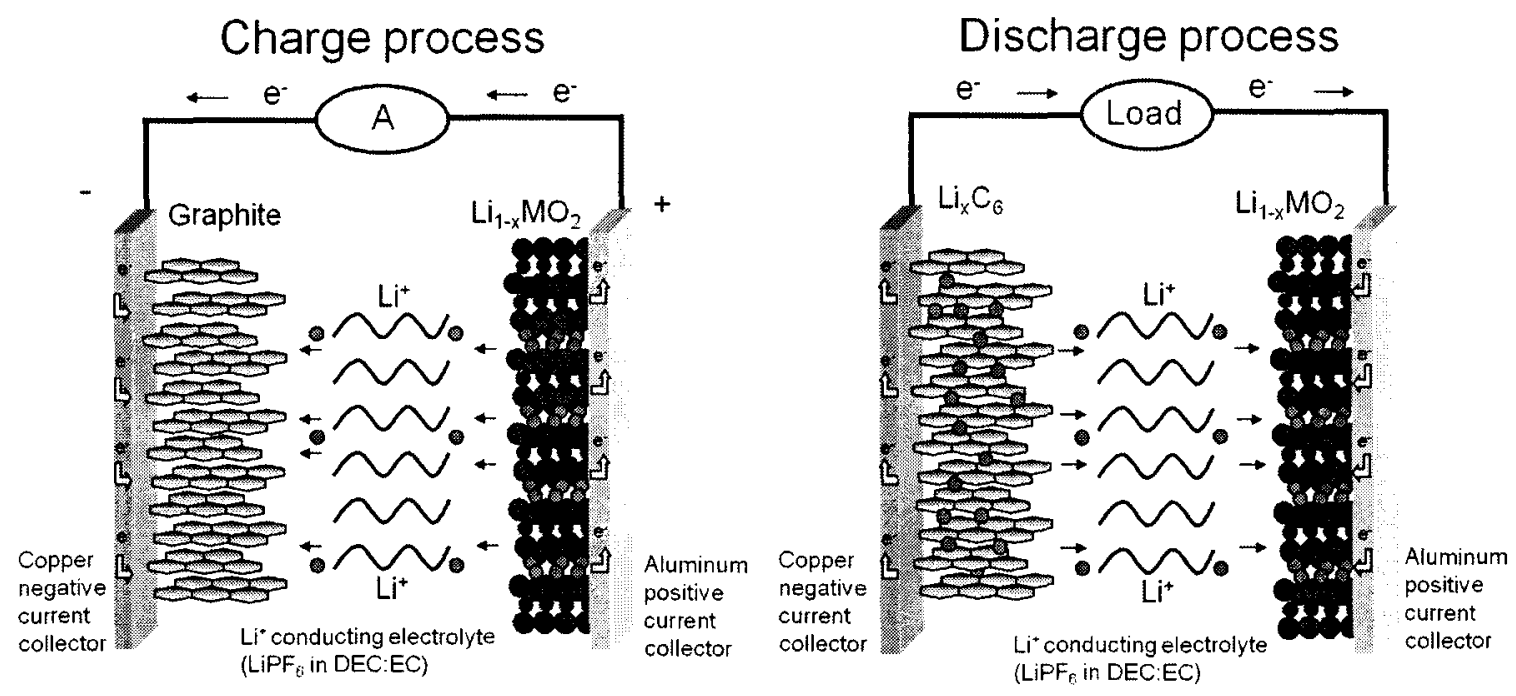

Figure 1.1: Charge-discharge mechanisms in LIBs

The discharge occurs when a load is connected to the external circuit and $\mathrm{Li}$ ions shuttle back to the cathode. The cell reactions taking place in battery comprising of carbon anode and lithium metal oxide (spinel) cathode are: 
Overall cell reaction: $\mathrm{LiMO}_{2}+6 \mathrm{C}=\mathrm{Li}_{(1-x)} \mathrm{MO}_{2}+\mathrm{Li}_{\mathrm{x}} \mathrm{C}_{6}$

At cathode: $\mathrm{LiMO}_{2}=\mathrm{Li}_{(1-\mathrm{x})} \mathrm{MO}_{2}+\mathrm{xLi}^{+}+\mathrm{xe}$

At anode: $6 \mathrm{C}+\mathrm{xLi}^{+}+\mathrm{xe}^{-}=\mathrm{Li}_{x} \mathrm{C}_{6}$

\subsubsection{Basic principles of operation in LIBs}

This section is an effort to acquaint the reader with the basic design aspects of electrodes in LIBs. A detailed study of the design principles has been presented elsewhere ${ }^{4}$. The power generated by a battery is the product of the generated current and the voltage of the battery. The voltage of the battery consists of $V_{o c}$, the open-circuit voltage of the battery which is given by the difference of electrochemical potentials of the cathode and anode indicated as $\mu_{C}$ and $\mu_{A}$, respectively and a voltage drop which is due to the internal resistance of the battery $R$. The voltage window available for a desirable electrolyte is directly related to the energy gap $(E)$ between the highest occupied molecular orbital (HOMO) and lowest unoccupied molecular orbital (LUMO) of the liquid electrolyte. The electrochemical potential of the anode $\mu_{A}$, which is also the anode Fermi level $\left(\square_{\mathrm{F}}\right)$ has to be lower than the liquid electrolyte LUMO to be stable thermodynamically in preventing the reduction of electrolyte by the anode. In addition, the electrochemical potential of the cathode $\mu_{C}$ or the Fermi level of the cathode $\left(\square_{\mathrm{F}}\right.$ ) has to be higher than the liquid electrolyte HOMO to be stable thermodynamically and prevent the oxidation of the electrolyte by the cathode. This is illustrated in Figure 1.2. In addition, the electrode potentials has to nearly match the electrolyte potential window, $E$ to obtain the maximum open circuit potential. 


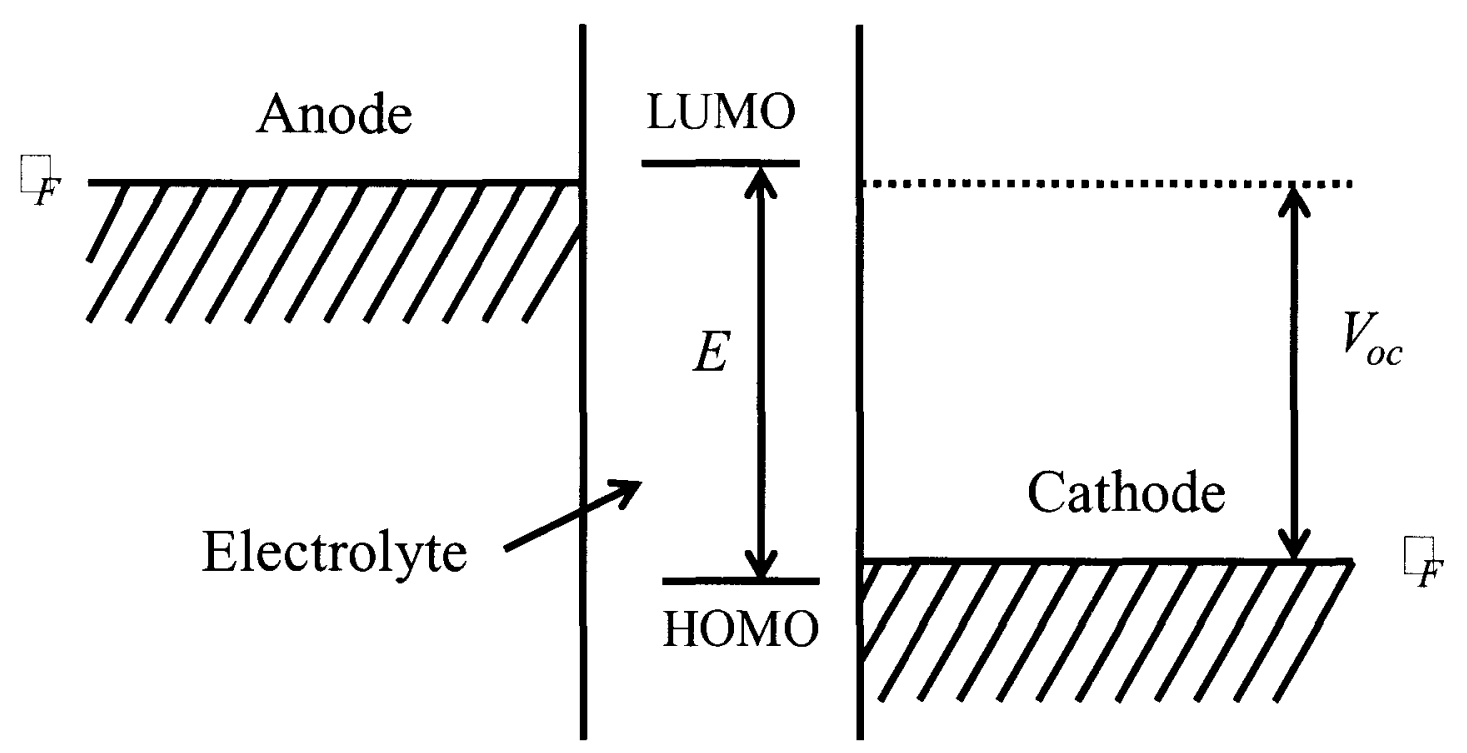

Figure 1.2: Electrolyte window with electrode electrochemical potentials. This figure has been recreated based on a figure in reference 4.

High voltage arises when the $V_{o c}$ is high as well as the internal resistance in a battery is low. The total internal resistance inside a battery is a sum of electrolyte resistance, interfacial resistance (electrolyte-electrode interfaces) and current collector resistance. In order to obtain high reversible capacity, high fraction of the electrode has to be utilized as a $\mathrm{Li}$ ion host and a good contact between the particles is necessary for the efficient electron transport which has to span over large number of charge-discharge cycles. If the material undergoes phase change and volume expansion during the reaction process, there is a high chance that the particles might fracture and hence, result in losing contact which in turn leads to a loss in electron conduction and ultimately, irreversible loss in capacity with cycling. If the electrode undergoes a two phase process during the reaction without volume expansion, the interface area decreases with the second phase penetration into the electrode. Upon reaching a critical interface area, some of the 
electrode particles might become inaccessible as the Li diffusion becomes slower than the sustained current. This can lead to diffusion limited reversible capacity loss with an increase in the current. In addition, this can also result in poor thermal conduction properties leading to excessive heat and hence, an unsafe battery operation. So, it is of utmost importance to consider all these aspects during the electrode design process.

Another important factor in choosing the material is the lithiation potential of the material with respect to $\mathrm{Li} / \mathrm{Li}^{+}$. The anode material lithiation potential has to be as low as possible whereas the cathode potential has to be as high as possible to obtain maximum voltage. In order to fully explore the voltage capacities of LIB, a non-aqueous electrolyte with a sufficiently large energy-gap window has to be used. Most of the electrolytes available for LIBs have a stable potential window lower than $5 \mathrm{~V}$, which also restricts the material of choice. Finally, it can be said that $\mathrm{Li}$ ion is more attractive than a proton because of the fact that lithium is more electropositive than hydrogen and hence, can give rise to a battery with high voltage. Similarly, all the other design aspects presented in the above discussion have to be taken into account for high capacity stable batteries. The next section provides a comprehensive look at different cathode and anode materials available for LIBs with their potentials and capacities.

\subsubsection{Electrode materials for LIBs}

There are a lot of different materials available as anodes and cathodes for LIBs. Detailed material characteristics have been presented in Figure 1.3. Towards the bottom of the Figure are the anode materials which have lower potentials w.r.t to $\mathrm{Li}^{+} / \mathrm{Li}$. The top

of the Figure represent the cathode materials with high potentials w.r.t to $\mathrm{Li}^{+} / \mathrm{Li}$. Graphite 
is the most widely used anode in commercial LIBs with a theoretical capacity of 372 $\mathrm{mAhg}^{-1}$. The next group of materials with theoretical capacities in the range of 400-600 $\mathrm{mAhg}^{-1}$ are the intermetallic alloys. Metal oxides are the next class of materials with significant promise as the future anodes in LIBs. There has been extensive research of these materials in the last 10 years. The metal oxides that are of great interest are the tin oxide $\left(\mathrm{SnO}_{2}\right)$, molybdenum oxide $\left(\mathrm{MoO}_{3}\right)$, cobalt oxide $\left(\mathrm{CoO} / \mathrm{Co}_{3} \mathrm{O}_{4}\right)$, iron oxide $\left(\mathrm{Fe}_{2} \mathrm{O}_{3} / \mathrm{Fe}_{3} \mathrm{O}_{4}\right)$, and titania $\left(\mathrm{TiO}_{2}\right)$ which have the highest capacities in the range of 800 $1400 \mathrm{mAhg}^{-1}$ and low potentials w.r.t to $\mathrm{Li}^{+} / \mathrm{Li}$.

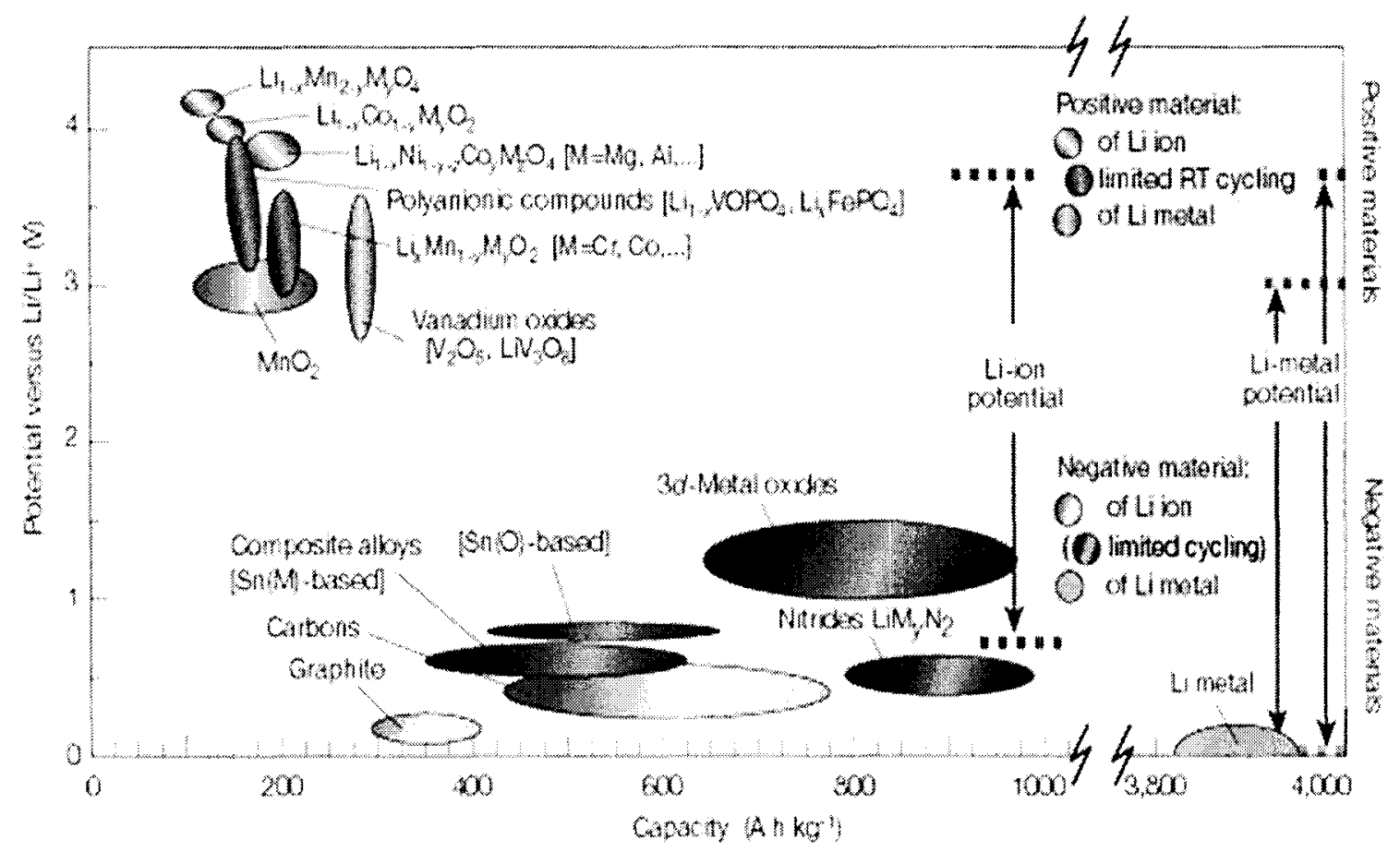

Figure 1.3: Electrode material characteristics of both anodes and cathodes. This figure is from reference 5 . 
The theoretical capacities of different metal oxides are presented in Table 1.1. The other inorganic anode materials with high capacities are silicon (Si), germanium (Ge) and tin (Sn) with their capacities shown in Table 1.1. Most the cathodes lie in the similar voltage ranges. The most widely used cathode is the $\mathrm{LiCoO}_{2}$. The other cathodes which grabbed significant attention are spinels including lithium manganese oxide $\left(\mathrm{LiMnO}_{2} / \mathrm{LiMn}_{2} \mathrm{O}_{4}\right)^{6}$ and lithium nickel oxide $\left(\mathrm{LiNiO}_{2}\right)^{7}$. Recently, it has been shown that a combination of these metals in these spinels (e.g. $\mathrm{LiCoM}_{\mathrm{x}} \mathrm{O}_{2}$ where $\mathrm{M}$ can be Ni, $\mathrm{Mn}$ etc., $\mathrm{LiMn}_{2} \mathrm{M}_{\mathrm{x}} \mathrm{O}_{4}$ where $\mathrm{M}$ can be $\mathrm{Co}$, Ni etc. $)^{8-10}$ help in attaining much higher potentials. Another interesting cathode material with good capacity and excellent stability with cycling is lithium iron phosphate $(\mathrm{LiFePO})$, the only drawback being lower potential than spinels and is also one of the upcoming commercial materials.

Table 1.1: Theoretical specific capacities of different materials.

\begin{tabular}{|c|c|c|}
\hline Material & Specific Capacity $\left(\mathbf{m A h g}^{-1}\right)$ & Potential Vs Li/Li ${ }^{+} \mathbf{~ V )}$ \\
\hline Silicon & 4200 & $<1.0$ \\
\hline Lithium metal & $\sim 4000$ & 0 \\
\hline Germanium & 1600 & $0.2-0.7$ \\
\hline Tin & 990 & $0.2-0.7$ \\
\hline Tin Oxide & 780 & $\sim 1.0$ \\
\hline Cobalt oxide & $\sim 900$ & $2.7-1.5 ;<0.7$ \\
\hline Molybdenum oxide & 1340 & $<1.0$ \\
\hline Iron oxide & 1440 & \\
\hline
\end{tabular}




\begin{tabular}{|c|c|c|}
\hline Titania & 165 & $\sim 1.5$ \\
\hline Graphite & 372 & $0.1 \sim 0.4$ \\
\hline
\end{tabular}

The overview in this section shows all the available materials for LIBs whereas the challenges associated with using these materials are presented in the next section.

\subsubsection{Challenges for LIBs}

The ever increasing demand for batteries in mobile and automotive applications requires the development of high capacity and stable batteries. Particularly, the batteries for EV/HEV/PHEV applications face extreme challenges with characteristics including high energy density and being able to withstand extreme weather conditions. High capacity retention, stability and high rate capabilities of the Li-ion batteries have attracted attention for its use in automotive applications, which will help us in developing vehicles with independent energy sources and devoid of dependence on foreign oil as well as reduce the carbon dioxide emissions in the environment preventing global warming. DOE targets for PHEV and HEV require operation of the vehicles in the temperature range of -32 and $52{ }^{\circ} \mathrm{C}$, and a calendar life of 15 years ${ }^{11,12}$. The performance and stability of carbonate electrolytes and present carbon anodes tend to drop significantly with temperature. The challenges associated with fabricating new-age high capacity stable batteries lies in identifying the stable electrolytes, new materials for both anodes and cathodes along with newer material architectures which can solve some of the problems of LIBs today. 
The anode side of the conventional batteries utilizes graphite and carbon based materials but these materials suffer from limited storage capacity and poor extreme temperature performance. However, physical and chemical modification of carbons can lead to capacities up to $\sim 500 \mathrm{mAhg}^{-1}$. The use of high capacity lithium metal nitrides $\left(600 \mathrm{mAhg}^{-1}\right)$ has proved to be alternative but their sensitivity to moisture has curtailed the scope of their manufacturing. The high capacity alternatives to carbons are the metal oxides and the inorganic semiconductor materials which also have preferential low lithiation potentials. But these materials undergo enormous volume expansions when lithiation occurs (e.g. $\mathrm{Si}-400 \%$ and $\mathrm{Sn}-260 \%$ ) which leads to mechanical fracture and instability. The volume expansions cause alleviated material stresses and strains which cracks the material leading to loss in electron conductive channels and in turn the capacity retention goes down with cycling. The material degradation can be curtailed by attaining shorter $\mathrm{Li}$ ion diffusion lengths, electron path lengths and materials which can relax strain associated with volume expansions. These excellent characteristics might be attained using nanoscale materials. Apart from the graphite and carbons, viable large scale synthesis reactors have yet to be developed for high performance materials which is one of the challenges to be overcome ${ }^{13}$.

The positive cathode contains the lithium that is necessary for the chargedischarge process. A voltage of $\sim 4 \mathrm{~V}$ makes $\mathrm{LiCoO}_{2}$ one of the most attractive cathode materials until the present day. $\mathrm{LiNiO}_{2}$ is another cathode material with capacity higher than that of $\mathrm{LiCoO}_{2}$. However, cycling can cause the collapse of $\mathrm{Li}_{x} \mathrm{NiO}_{2}$ (lithium taken out from the spinel structure) leading to the exothermic reaction with the electrolyte causing concerns for the safe operation of the batteries. The spinel framework can be 
stabilized by the addition of divalent, trivalent or tetravalent substitute (gallium, magnesium and aluminum) which are inert electrochemically. The other materials with

tremendous promise are $\mathrm{LiFePO}_{4}$ and $\mathrm{Li}_{\mathrm{x}} \mathrm{VO}_{2}{ }^{14,15}$ compounds. These characteristics can be further improved by tuning the electrode morphology and reducing the dimensions to nanoscale. However, the reactivity of the nanoscale materials with the electrolyte is unknown to a large extent and has to be studied to make them viable for LIBs ${ }^{13}$.

In terms of the electrolytes, the most widely used electrolyte is lithium hexaflourophosphate $\left(\mathrm{LiPF}_{6}\right)$ in ethylene carbonate (EC) and diethyl carbonate (DEC). However, investigations are underway to identify new electrolytes which can sustain extreme temperatures and operate in a safe environment. Presently, solid electrolytes have attracted considerable attention due to their safe operation over a wide temperature range. The present challenges in LIBs for both the anodes and the cathodes include finding the best suited materials and material architectures which can help overcome the problems of instability. New electrolyte materials including solid materials need to be explored to increase their stability.

\subsection{Motivation for this study}

Since its first introduction by Sony in 1991, Li-ion batteries have seized the highperformance rechargeable-battery market. The stability and high cycling rate capabilities of the Li-ion batteries along with high energy densities has attracted attention toward their use in automotive applications. However, the wide-spread use of lithium ion battery technology in applications other than small rechargeable batteries requires a step change 
improvement in electrode and electrolyte materials in terms of capacity, durability and safety.

The stability and high cycling rate capabilities of the Li-ion batteries along with high capacities has attracted attention for its use in automotive applications which will help us in developing vehicles with independent energy sources (built-in) and devoid of dependence on foreign oil as well as reduce the carbon dioxide emissions in the environment, preventing global warming. This requires us to explore ways to improve the capacities and stability along with the electronic properties of the anode material system. This can be tackled in two ways: the first one is to find new materials which have good inherent capacity retention and stability; the other one is to modify the morphology of the already available high capacity materials to make them more efficient. Hence, the primary motivation is to find the high capacity material with a low potential w.r.t Li/Li ${ }^{+}$ and the stable architecture for the material or material composite to design electrode materials with high energy density.

\subsection{Objectives of this study}

In order to attain high capacity retention with durability, one needs to think beyond conventional graphitic or carbon electrodes and explore ways to improve the stability by the architectural modification of already available high capacity materials to make them more efficient. In this context, 1-dimensional nanowires have been shown to have higher surface area to volume ratios than thin films. In addition, the onedimensional materials potentially offer smaller length scales for lithium intercalation (diameter) and higher charge transport characteristics and strain accommodation for 
better mechanical properties. The nanowire powder based thin films is also expected to require low sintering temperatures which should make them amenable for roll to roll coating processes on plastic substrates. However, there is little or no information available in terms of charge transport and mechanical durability during lithium intercalation/de-intercalation. In the case of many systems, it may be necessary to design hybrid structures involving both 1-D structures as they do not pack well for high density thin films. Also, hybrid structures are also needed for adding multiple functionalities toward designing both durable anode and cathode materials. Thus, in this dissertation, we propose a novel, generic, hybrid architecture involving nanowires and nanoparticles which can help us achieve high capacity and stability for a variety of materials systems.

Figure 1.4 illustrates the way thin films and nanoparticles fail with lithium intercalation/de-intercalation due to mechanical fracture thus losing the active area and conduction resulting in capacity fade. Nanomaterials especially 1-dimensional nanowires offer unique characteristics such as high surface area per unit volume, better strain relaxation, efficient $1 \mathrm{D}$ electron conduction pathways and fast charge transport which reduces the mean lengths of $\mathrm{Li}$ ion diffusion, provides larger active area for lithiation increasing the capacity retention and sustaining high charge-discharge rates. These aspects have been clearly illustrated in Figure 1.4. However, there is not much information available about the cycling behavior of 1-D materials as lithium ion battery electrodes. In this dissertation, we propose a generic concept of hybrid architectures involving one dimensional nanowires coated with nanoparticles for designing high capacity and durable anodes. So, one of the objectives is to fully understand cycling behavior of pure phase $\mathrm{SnO}_{2}$ nanowires, carbon microtubes (CMTs), molybdenum oxide 
$\left(\mathrm{MoO}_{3}\right)$ nanowires and tungsten oxide $\left(\mathrm{WO}_{3}\right)$ nanowires which potentially could be used for 1-D materials in our hybrid architectures.

Thin film/Nanoparticle films

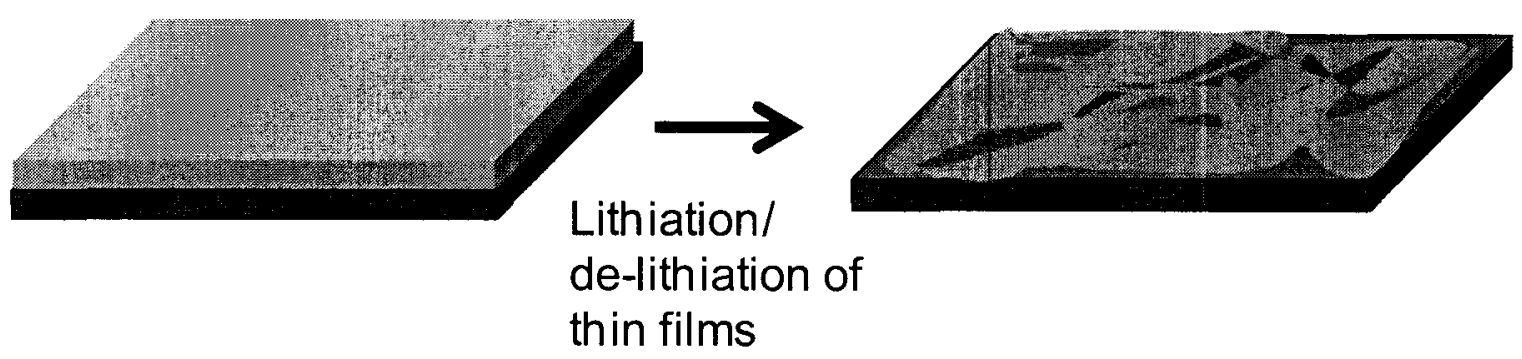

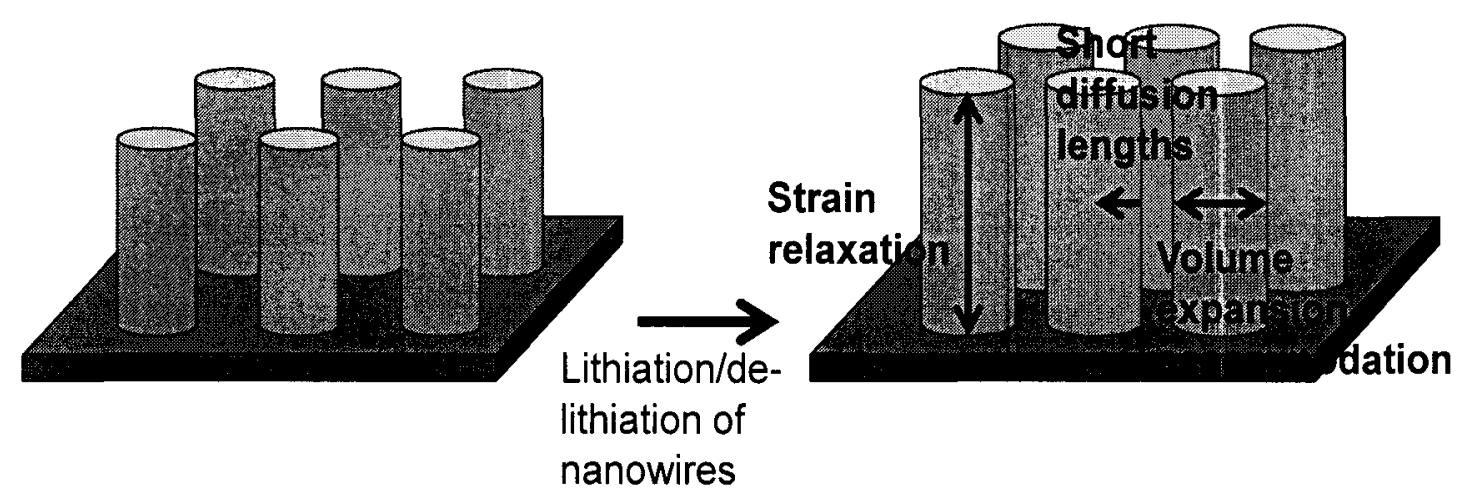

Figure 1.4: Schematic showing the strain, volume accommodation in 1D nanowires whereas the thin films/nanoparticles undergo mechanical fracture.

The specific goals for this project are as following:

a. Fundamental understanding about cycling behavior of nanowire thin films and arrays: 1-D nanowires are quite interesting as electrode materials because of all the interesting characteristics discussed earlier. Nanowires can be used as both 
powders to fabricate electrode mats and as-grown directly on conducting substrates eliminating the need for a binder. It is of utmost interest to understand the lithiation characteristics of 1-D nanomaterials as few studies are available. The performance of these materials has been evaluated electrochemically to understand the fundamental aspects of lithiation and de-lithiation.

b. Concept of hybrid architectures and their cycling behavior: The concept of hybrid structures is to have nanowires or nanotubes as a base material which can support the growth of other nanoparticles or nanowires on their surfaces to create a 2 dimensional hybrid structure in which the primary purposes of the base are to provide the electronic conductivity and material stability, whereas the surface structures provide the majority of the capacity. The surface materials are spaced well apart to accommodate the volume expansion of the materials with lithiation and de-lithiation. The spacing also exposes the surface of the underlying material which can also serve as additional sites for $\mathrm{Li}^{+}$intercalation. In addition, the 2dimensional architecture gives rise to additional surface area depending on the base diameters and the structures on the base surface, the separation between the surface structures and the lengths of the base nanowires. The base materials can comprise of various metal oxide materials like $\mathrm{MoO}_{3} / \mathrm{SnO}_{2}$ and carbon microtubes/nanotubes. The surface of these materials can then be decorated with nanoparticles/nanowires of high capacity materials like Sn, Si and Ge. The hybrid architectures being powders, exhibit better efficiency and can be synthesized in large quantities. 
c. Lithiation characteristics of nanoscale and hybrid architectured materials: To our knowledge, there have been no studies on the lithiation characteristics as a function of particle size. In this study, we aim to understand the lithiation characteristics of the 1-D nanowires and hybrid architectures. The study of these characteristics as a function of particle sizes will enable us to better understand the nanoscale behavior of the materials along with thorough understanding of the performance of different material architectures.

\subsection{Dissertation outline}

The dissertation has been organized into eight chapters. Chapter one introduces the global energy demand, energy storage perspectives, LIB working and design principles, challenges in LIBs and fundamental objectives of this study. Chapter two is an in-depth understanding of different nanomaterials that have been evaluated as potential anode materials, their performance, significance and the future direction with those materials. Chapter three describes in detail the synthesis techniques, electrode fabrication processes and both the physical and electrochemical characterization techniques. Chapter four is the application of $\mathrm{SnO}_{2}$ nanowires as potential anode candidate. Modifications of the architectures and their drastic improvements are the heart of the chapter. Chapter five advances the study in chapter four for the basic understanding of the lithiation characteristics in nanoscale architectures and then, a comparative study with macroscale particles emphasizing the nanoscale merits. Chapter six introduces the carbon microtubes and their performance characteristics along with extensive post-lithiation analysis to understand their increased performance. Chapter seven details the need for the array 
configuration using metal oxides molybdenum oxide and tungsten oxide as potential anode candidates. The conclusions and suggestions for future directions have been presented in chapters eight and nine, respectively. 


\section{CHAPTER 2}

\section{BACKGROUND}

\subsection{Introduction}

This chapter details the evolution of the energy storage systems from a historical and technological perspective. The primary focus is the anode material advancements in lithium ion batteries (LIBs). In particular, with the advent of nanotechnology, the use of these materials has been shown to improve the performance of the battery compared to the micron sized materials. The review primarily focuses on the use of carbon based materials, metal oxides, inorganic semiconducting materials and hybrid 3-D architecture materials as anodes and their performance dependence on material architectures. In the case of metal oxide materials, it has to be noted that majority of the work until the start of this project is focused on thin films and nanoparticles and that, the studies pertaining to the use of metal oxide nanowires as anode materials have been limited. Most of the work discussed in this chapter has been performed during the course of this project.

\subsection{Electrochemical energy storage systems}

In addition to battery technology, there are other energy storage technologies available. They are pumped hydro, flywheels, ultracapacitors and chemicals such as hydrogen. The advantages, disadvantages and applications of various energy storage technologies are presented in Table 2.1. 
Table 2.1: Comparison of various energy storage technologies. This table is adapted from reference 16 .

\begin{tabular}{|c|c|c|}
\hline $\begin{array}{c}\text { Energy storage } \\
\text { system }\end{array}$ & $\begin{array}{c}\text { Use in power } \\
\text { applications }\end{array}$ & $\begin{array}{c}\text { Use in energy } \\
\text { applications }\end{array}$ \\
\hline Compressed Air & - & High \\
\hline Flow batteries & Medium & High \\
\hline Lead acid batteries & High & Low \\
\hline Nickel - cadmium batteries & High & Medium \\
\hline Lithium ion batteries & High & Low \\
\hline Lithium air batteries & High & Low \\
\hline Metal air batteries & - & High \\
\hline Lithium sulfur batteries & High & High \\
\hline Sodium sulfur batteries & High & High \\
\hline Flywheels & High & Low \\
\hline $\begin{array}{c}\text { Superconducting magnetic } \\
\text { storage systems }\end{array}$ & High & - \\
\hline Capacitors & High & Medium \\
\hline
\end{tabular}

Table 2.1 clearly indicates that batteries are of extreme interest among the numerous energy storage systems, because of their high power densities and reasonable energy densities. 


\subsubsection{History of storage systems}

Energy storage systems have come a long way starting from the $16^{\text {th }}$ century. This section is devoted to their evolution and the present day scenario. A detailed history has been presented elsewhere ${ }^{17}$. The most important advancements in batteries have been presented in this section.

Gilbert has first established the concept of electrochemistry and its study in 1600 . But it wasn't until 1800 that the first electrochemical device was invented. Alessandro Volta created the first ever battery using two metals zinc and copper separated by blotting papers in acidic medium of sulphuric acid. His battery proved the theory that electric current is generated when metal and chemicals come into contact and the voltage varies with the materials used. He asserted the fact that increasing the number of individual metal stacks increases the overall voltage. His idea of converting the chemical energy in to electrical energy forms the basis for all the batteries. However, this battery has low capacity for its weight and is unsafe to operate due to the generation of hydrogen bubbles. In order to overcome some of these problems, Daniell cell was invented in 1836 . This cell consists of $\mathrm{Cu}$ and $\mathrm{Zn}$ electrodes in separate copper sulfate and zinc sulfate solutions respectively. At the anode, $\mathrm{Zn}$ oxidizes to give electrons which flow through an external circuit to the $\mathrm{Cu}$ cathode. The salt bridge transports the ions to balance the charge.

Fuel cell was invented by Grove in 1839 which resembles the present day phosphoric acid fuel cells. The invention of the lead acid battery by Plante in 1859 followed by the invention of nickel-cadmium battery by Jungner in 1899 . The Ni-Cd battery offered better capacity with weight than their counterpart lead-acid batteries and were researched for further improvements in their performance. Iron was studied to 
replace cadmium but was inefficient. Commercial production of these batteries started in 1906 in Sweden. These batteries were quite popular despite their higher cost and thermal runaway problems which can compromise safety. The next 50 years did not see major advancements until mid 1950's when Lewis Urry first invented the modern day alkaline battery consisting of $\mathrm{Zn}$ anode and manganese dioxide cathode in an alkaline medium which hit the markets in 1959. The first commercial nickel metal hydride (Ni-MH) was introduced in 1989 with longer life spans and better safety (no cadmium presence) than Ni-Cd batteries. In 1970s, Whittingham ${ }^{18}$ first developed lithium ion battery (LIB) with lithium metal as the anode. The capacity was high but recharging the battery created $\mathrm{Li}$ plating which ultimately touches the cathode to cause a short circuit. They were hence, considered unsafe. Subsequently carbon based intercalation anodes were developed with improved cycleability. Goodenough ${ }^{19,20}$ has led a research team and identified manganese oxide spinel with polyanions as the cathode leading to its introduction by Sony in 1991 which has forever changed the battery market.

\subsubsection{Overview of different battery system characteristics}

A battery can be primarily assessed based on the characteristics like energy density, cycle life etc. These characteristics of different battery systems have been presented in Table 2.2. Energy densities of different battery systems are presented in Figure 2.1. Although, Li metal anode exhibits high energy densities, it is highly unsafe for application in rechargeable batteries. $\mathrm{Li}$ ion batteries stand out in terms of weight, energy density and cycle life among all others. 
Table 2.2: Performance characteristics of different battery types.

\begin{tabular}{|c|c|c|c|c|}
\hline & Ni-Cd & Ni-MH & Lithium & Li-ion \\
\hline $\begin{array}{c}\text { Energy } \\
\text { Density }\end{array}$ & Low & Moderate & $\begin{array}{c}\text { Extremely } \\
\text { High }\end{array}$ & High \\
\hline Cycle Life & High & Low & Extremely low & High \\
\hline Weight & Heavy & Heavy & Light & Light \\
\hline $\begin{array}{c}\text { Memory } \\
\text { Effect }\end{array}$ & Yes & Low & No & No \\
\hline Toxicity & High & Low & Low & Low \\
\hline
\end{tabular}

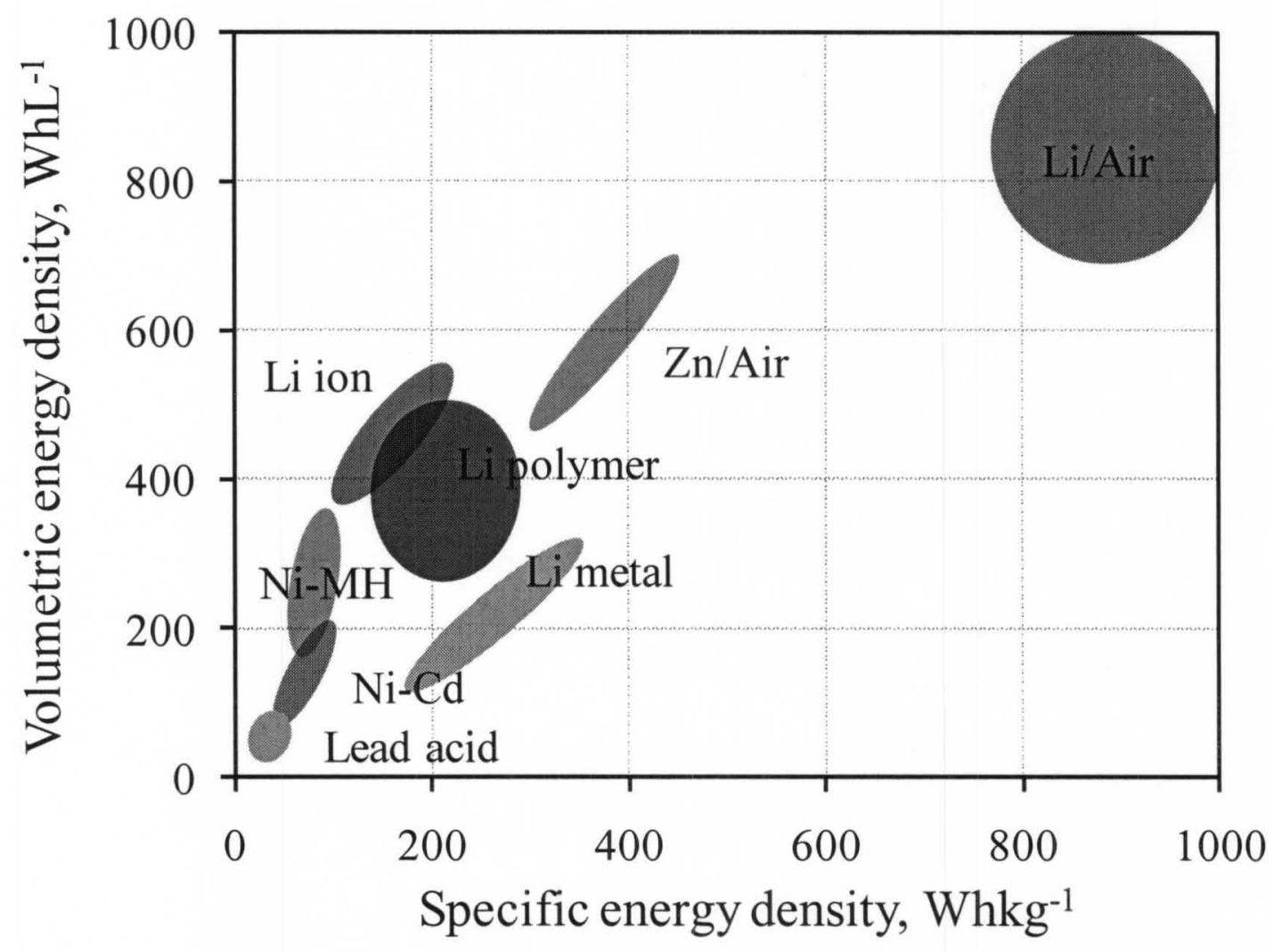

Figure 2.1: Energy density of different battery systems. 


\subsection{Carbon based materials as anodes in LIBs}

Carbon based materials have been the most widely used anodes for LIBs. The significant advantages of carbon based materials over polymers, metal oxides and semiconductors include: (a) low redox potential with respect to (w.r.t) to $\mathrm{Li} / \mathrm{Li}^{+}$which means a high potential w.r.t the cathode material and high voltage battery. (b) Superior cycling performance because of good mechanical stability. (c) High reactivity and considerably safe under regular operating conditions. (d) Inexpensive synthesis of carbonaceous materials. (e) Low environmental impact during use in the battery electrode and disposal. Lithium ions are inserted into the solid carbon host as indicated by the following reaction ${ }^{21,22}$

$$
x L i^{+}+x e^{-}+C_{y} \leftrightarrow L i_{x} C_{y}
$$

During the charge process, the lithium is incorporated into the carbon host which is also called intercalation forming $\mathrm{Li}_{\mathrm{x}} \mathrm{C}_{\mathrm{y}}$. Carbon host material also accepts the corresponding negative charges exhibiting both electronic and ionic conductance. The discharge process termed as de-intercalation reversibly removes $\mathrm{Li}^{+}$ions from the host material. Carbonaceous materials used as anodes for LIBs can be classified as graphitic carbons and non-graphitic carbons. The number of $\mathrm{Li}^{+}$ions that can be intercalated and the electrochemical characteristics vary based on the type of carbon used.

\subsubsection{Graphitic carbons as anodes for LIBs}

Graphite consists of interconnected hexagonal planes with six carbon atoms on the vertices of hexagon in a single plane ${ }^{23}$. The hexagonal planes are in turn held by weak Van der Waals forces with an interlayer spacing of $3.354 \mathrm{~A}^{\circ}$. The lithium ions intercalate 
into the spacing between the layers with maximum of one $\mathrm{Li}^{+}$ion per six carbon atoms $\left(L i_{x} C_{6}: x \leq 1\right)^{23}$ corresponding to a theoretical capacity of $372 \mathrm{mAhg}^{-1}$. Any additional $\mathrm{Li}$ intercalation through the basal plane happens at the defect sites. The volume expansion of graphite on $\mathrm{Li}$ intercalation is $10.3 \%$, much lower than other material systems which make it mechanically stable and undergo low capacity loss with repeated cycling ${ }^{24,25}$. Rüdorff and Hoffman have proposed the concept of graphite based rechargeable cell in $1938^{26}$. The practical use of lithium intercalation into graphite has been investigated by Armand and Touzain in $1977^{27}$. Several researchers have studied the mechanism of lithium intercalation into graphite $e^{28-31}$. Winter et $a 1 .^{23}$ have shown that graphite can intercalate higher $\mathrm{Li}$ than the theoretical maximum during the first cycle but the deintercalation results in extraction of only up to $80 \%$. Further cycling of the electrode leads to $100 \%$ extraction of the material.

\subsubsection{Multi walled carbon nanotubes}

Multi walled carbon nanotubes (MWCNTs) are one of the first nanoscale carbon materials to be investigated which show good capacity and stability. Frackowiak et al. ${ }^{32}$ have shown that MWCNTs obtained by acetylene catalytic decomposition at $900^{\circ} \mathrm{C}$ retain a high initial capacity of $952 \mathrm{mAhg}^{-1}$. However, these electrodes suffer a capacity loss of $505 \mathrm{mAhg}^{-1}$ during the first cycle and retain $273 \mathrm{mAhg}^{-1}$ after five cycles. In this case, the irregularities on the walls of the tube are believed to trap lithium and give rise to large capacity losses. The use of highly crystalline MWCNTs with closed ends as anodes has led to low reversible capacities of $125 \mathrm{mAhg}^{-1}$ suggesting that the closed ends hinder the $\mathrm{Li}$ diffusion into the interior of the tubes and the layers of the MWCNTs ${ }^{33}$. In another 
study, open ended MWCNT arrays with diameters on the order of $\sim 100 \mathrm{~nm}$ have shown capacity retention of $490 \mathrm{mAhg}^{-1}$ with an improved performance ${ }^{34}$. MWCNTs synthesized by several different approaches and used as anodes have shown capacity retentions in the range of $100-540 \mathrm{mAhg}^{-1}$ along with high irreversible capacities ${ }^{33,35-37}$. In order to further improve the performance of MWCNTs, Wang et al. ${ }^{38}$ have utilized a novel technique to cut the MWCNTs and make them shorter in lengths. During this process, nickel (Ni) particles deposited and oxidized on the MWCNT surface are heated to $900{ }^{\circ} \mathrm{C}$ which results in the breaking of CNTs. Finally, the metal particles are then eliminated which leaves behind the pure short CNTs which are $200 \mathrm{~nm}$ in length and have visible surface defects. These tubes retain capacity higher than $400 \mathrm{mAhg}^{-1}$ at current density of $25 \mathrm{mAg}^{-1}$ after 50 cycles. Although all the above studies have shown good capacity retention, there is a further need to improve the material capability to be applicable in HEVs, hybrid power plants and portable power sources.

\subsubsection{Single walled carbon nanotubes}

In this context, single walled carbon nanotubes (SWCNTs) with open ends have shown enhanced performance. SWCNTs synthesized using dual pulsed laser vaporization with diameters in the range of $10-50 \mathrm{~nm}$ were purified and used as anodes for LIBs ${ }^{39}$. These anodes show reversible capacities close to $460 \mathrm{mAhg}^{-1}$, which amounts to $1.23 \mathrm{Li}^{+}$ ions per six carbon atoms. This material however, exhibits large first cycle irreversible capacity loss of $1200 \mathrm{mAhg}^{-1}$ which is due to the high surface area. Other studies using free-standing nanotube electrodes indicate capacities in the range of $400 \mathrm{mAhg}^{-1}$ which is similar to the performance of MWCNTs ${ }^{40,41}$. Frackowiak and Beguin ${ }^{42}$ have synthesized 
and tested SWCNTs and MWCNTs with capacity retention in the range of $220 \mathrm{mAhg}^{-1}$ to $780 \mathrm{mAhg}^{-1}$ with an irreversible capacity of 460 to $1080 \mathrm{mAhg}^{-1}$. However, SWCNTs synthesized by laser ablation at $1150^{\circ} \mathrm{C}$ using $\mathrm{Ni} / \mathrm{Co}$ catalyst and a graphite catalyst showed an initial reversible capacity of $681 \mathrm{mAhg}^{-1}$ which amounts to $1.8 \mathrm{Li}^{+}$ions per six carbon atoms. Further etching of these tubes enhanced the reversible capacity to $\sim 1000$ $\mathrm{mAhg}^{-1}$ corresponding to $\mathrm{Li}_{2.7} \mathrm{C}_{6}$ and resulted in low capacity loss with cycling ${ }^{43}$. The etched tubes have increased $\mathrm{Li}$ uptake due to the filling of the nanotube interior through open ends and additional defect sites on the nanotube surfaces ${ }^{44}$. Increased $\mathrm{Li}$ intercalation has also been observed in ball-milled SWCNTs when the nanotubes have been fractured using mechanical methods ${ }^{45}$. SWCNT-vanadium oxide $\left(\mathrm{V}_{2} \mathrm{O}_{5}\right)$ composite electrodes have exhibited capacities above $400 \mathrm{mAhg}^{-1}$ at discharge rates as high as 1200 $\mathrm{mAg}^{-1}$ and good stability with cycling ${ }^{46}$.

\subsubsection{Carbon nanofibers}

In terms of the structure, carbon nanofibers (CNFs) have graphitic hexagonal basal planes parallel to the fiber axis with a ring texture along the cross section. The planar structure results in exceptional structural properties along with good thermal and electronic conductivity ${ }^{47,48}$. CNFs have been primarily synthesized in vapor phase by the decomposition of hydrocarbons in the presence of transition metal particle catalyst at temperatures of $1000-3000^{\circ} \mathrm{C}^{49}$, 50. Another synthesis technique showing tremendous promise for mass production is the floating reactor method. In this process, hydrocarbons and catalytic particles derived from compounds such as ferrocene, are dispersed threedimensionally in a reaction chamber to produce uniform fibers of diameters $100-200$ 
$\mathrm{nm}$ with high yield. CNFs synthesized by the floating reactor method and graphitized have shown a capacity retention of $>250 \mathrm{mAhg}^{-1}$ at a current density of $0.2 \mathrm{mAcm}^{-2}$ for up to 230 cycles $^{51}$. It has been shown that the boron doping of the CNFs creates electron acceptor levels which in turn show superior performance than pristine graphitized $\mathrm{CNFs}^{52}$.

Kim et al. ${ }^{53}$ have synthesized CNF webs by polymer solution electrospinning. In electrospinning, a polymer solution held in a capillary tube is subjected to a high electric field which pulls the solution in the form of cone. As the electrical forces keep accelerating the cone, it forms a jet which is collected onto a metal screen. The high surface area, highly conductive and mechanically stable pure CNF webs show reversible capacity of $450 \mathrm{mAhg}^{-1}$. In another study, porous CNFs with diameters of $100 \mathrm{~nm}$ showed reversible capacity of $400 \mathrm{mAhg}^{-1}$ at $0.5 \mathrm{C}$ rate ${ }^{54}$. The high rate capability is attributed to high porosity and the 1-dimensional electronic conductivity. Hollow carbon fibers have also been synthesized by heating poly(styrene) with using iron as the catalyst $^{55}$. The fibers have graphene structure and contain iron in the hollow tubular

structure. These fibers retain a capacity of $860 \mathrm{mAhg}^{-1}$ at current density of $0.186 \mathrm{Ag}^{-1}$. Another study using CNFs synthesized by floating catalyst method and used as anodes showed reversible specific capacity of $461 \mathrm{mAhg}^{-1}$ at $0.1 \mathrm{C}$ rate and sustained high charge-discharge rates with good reversible capacity ${ }^{56}$.

\subsubsection{Other carbon based materials}

Graphene nanosheets (GNS) are another class of materials one-atomic layer thick and are two-dimensional layers with bonded carbon networks. Their excellent 
conductivity, high surface area of $2600 \mathrm{~m}^{2} / \mathrm{g}$ and broad electrochemical window makes them attractive for LIBs. GNS when used as anodes showed capacity retention of 290 $\mathrm{mAhg}^{-1}$ at current density of $0.05 \mathrm{Ag}^{-1}$ after $20 \mathrm{cycles}^{57}$. The capacity of GNS increased to $600 \mathrm{mAhg}^{-1}$ at similar current density when mixed with fullerenes $\left(\mathrm{C}_{60}\right)$ and was proposed that the d-spacing of the GNS plays a role in the enhanced capacity retention. A study using natural graphite flakes coated with carbon exhibited capacity retention of 378 $\mathrm{mAhg}^{-1}$ with columbic efficiency of $100 \%$ for up to 100 cycles $^{58}$. The excellent capacity retention is attributed to increased interlayer distance caused by mechanical means and the porous structure which accommodates the volume expansion caused by the lithium intercalation and de-intercalation.

An artificial graphitized carbon termed graphitized carbon nanobeads (GCNBs) has been developed to sustain high-rate use ${ }^{59}$. GCNBs exhibited low capacities of $\sim 200$ $\mathrm{mAhg}^{-1}$ at low rates, which is lower than other graphitized materials but these materials sustain capacities of up to $\sim 180 \mathrm{mAhg}^{-1}$ even at rates as high as $12 \mathrm{C}$ (the charge rate in this case is $\mathrm{C} / 6$ ), which is higher than other graphitized material capacities. It is postulated that in the case of GCNBs, the surface is covered with basal planes instead of the edge planes which results in significantly lower decomposition of the electrolytes and hence, smaller resistance due to the SEI formation resulting in excellent rate performance. Carbon nanosprings (CNSs) were synthesized by chemical vapor deposition with spring diameter and carbon ring diameter of $\sim 150 \mathrm{~nm}$ and $\sim 100 \mathrm{~nm}$ respectively ${ }^{60}$. CNSs show capacity retentions of $\sim 440 \mathrm{mAhg}^{-1}$ and $\sim 160 \mathrm{mAhg}^{-1}$ at current densities of $50 \mathrm{mAg}^{-1}$ and $3 \mathrm{Ag}^{-1}$. This capacity is retained for up to 100 and 200 cycles with minimal loss at $50 \mathrm{mAg}^{-1}$ and $3 \mathrm{Ag}^{-1}$ respectively ${ }^{61}$. In this case, nanometer sized carbon blocks 
enhance the lithium kinetics in the material which results in the high rate performance. In addition, the unusual spring morphology provides mechanical flexibility volume expansion/relaxation relieving the associated strain in the material.

Graphitic carbon materials presented in this section have shown good capacity retention with cycling. However, most of the electrodes have limited rate capability. Only a few materials exhibit good rate capability at rates lower than that used for the HEVs, PHEVs and grid stabilization applications.

\subsubsection{Non-graphitic carbons as anodes for LIBs}

Non-graphitic carbons are similar to graphitic carbons in terms of planar hexagonal arrangement of carbon atoms but are not ordered in the c-direction ${ }^{62,63}$. Nongraphitic carbons are comprised of amorphous areas which arise due to the incomplete hexagonal plane edges as well as graphitic plane cross linking to a random degree and are characterized by the extent of these two features ${ }^{62,63}$. The extent of these features depends on the synthesis temperature and the type of precursor used for the synthesis ${ }^{64}$. Most of the non-graphitic carbons are synthesized by hydrocarbon pyrolysis at temperatures below $1500^{\circ} \mathrm{C}$. The lithium intercalation mechanism into non-graphitic carbons differs drastically from that of graphitic carbons. Intercalation of $\mathrm{Li}^{+}$ions starts at potentials higher than $1 \mathrm{~V}$ and following a continuous slope unlike in graphite in which intercalation happens at potentials lower than $0.4 \mathrm{~V}$ and forms distinguishable plateaus for each stage of intercalation ${ }^{65,66}$. These can be further divided into "soft" and "hard" carbons $^{62}$. The ones that graphitize on heating to temperatures in the range of $1500-$ $3000^{\circ} \mathrm{C}$ are termed as the "soft" carbons which finally form the graphite-like crystallites 
while the carbons that do not graphitize are termed as the "hard" carbons. The high specific charge non-graphitic carbons can intercalate high amount of lithium than graphite, with $\mathrm{x}=1.2$ to 5 in $L i_{x} C_{6}$ depending on the synthesis conditions ${ }^{64}$. Several nongraphitic carbons have been studied to develop high capacity stable carbonaceous anode materials.

A type of disordered carbon was synthesized by the pyrolysis of precursors such as hexa(phenyl)benzene (HPB) and hexakis(p-bromophenyl)-benzene (HPB-Br) ${ }^{67}$. The as-synthesized carbon samples were ball-milled to obtain better shape uniformity. These electrodes show an initial capacity of over $1000 \mathrm{mAhg}^{-1}$ which reaches a steady 550 $\mathrm{mAhg}^{-1}$ for up to 200 cycles at a rate of $\mathrm{C} / 5^{68}$. In this particular electrode, the high initial irreversible capacity is followed by significantly low capacity loss with further cycling which makes this a high performance electrode. In addition, this particular electrode was used to fabricate a battery with $\mathrm{LiMn}_{2} \mathrm{O}_{4}$ as the cathode which exhibited stable capacity of $>400 \mathrm{mAhg}^{-1}$ and stable energy density of $\sim 150 \mathrm{Whkg}^{-1}$ for up to 100 cycles at a charge rate of $\mathrm{C} / 5$ indicating great promise as an electrode material ${ }^{68}$. In another study, carbon anodes were synthesized using cotton and phenol cloth precursors ${ }^{69}$. Carbon activation by oxidation leads to highly porous structure but these pores lead to large irreversible capacity due to SEI formation and double layer capacitance. Chemical vapor deposition treatment of the carbon surfaces makes the pores selective to passage of $\mathrm{Li}^{+}$ions and hinder the reaction with the electrolytes. The theoretical capacity of these carbon electrodes is evaluated to be $\sim 1400 \mathrm{mAhg}^{-1}$, whereas the material retains $10 \%$ of the capacity with $\sim 80 \%$ efficiency. However, further process optimization is required to improve the performance of the activated carbons. 
Ryoo et al. ${ }^{70}$ have first reported the synthesis of mesoporous carbon using a silica template by carbonizing sucrose which have attracted as potential candidates for hydrogen storage and LIBs. Ordered mesoporous carbon (CMK-3) was synthesized using silica (SBA-15) as the template ${ }^{71}$. CMK-3 exhibits an excellent first cycle capacity of $3100 \mathrm{mAhg}^{-1}$ corresponding to $8.4 \mathrm{Li}^{+}$ions per 6 carbon atoms. However, the second cycle capacity is $\sim 1100 \mathrm{mAhg}^{-1}$ with an irreversible capacity of $2000 \mathrm{mAhg}^{-1}$ mainly due to the formation of SEI layer. CMK-3 retains a final capacity of $850-900 \mathrm{mAhg}^{-1}$ after 20 cycles which accounts to $2.3-3 \mathrm{Li}^{+}$ions per 6 carbon atoms. The average capacity loss over the first 20 cycles is also a meager $1.3 \%$ per cycle. The good electrochemical characteristics are attributed to the high surface area $\left(1030 \mathrm{~m}^{2} \mathrm{~g}^{-1}\right)$ and the uniform mesopore diameter of $3.9 \mathrm{~nm}$.

\subsection{Metal oxide electrodes as anode materials}

Metallic lithium exhibits exceptional capacity of $\sim 4000 \mathrm{mAhg}^{-1}$ but when used in rechargeable batteries undergoes lithium plating or the dendritic growth of lithium which can extend to the cathode creating a short circuit leading to unsafe conditions (fire). In this context, Dey ${ }^{72}$ has first demonstrated in 1971 that lithium can reversibly form alloys with metals which can give rise to capacities higher than that of graphite. The electrochemical reaction can be represented as follows:

$$
x L i^{+}+x e^{-}+M \leftrightarrow M L i_{x}
$$

Although the alloying looks straightforward, it still involves complex processes of several intermediate intermetallic phase formations $\left(\mathrm{MLi}_{\mathrm{x}}\right)$. Metals that have been studied 
in detail include tin $(\mathrm{Sn})$, cobalt $(\mathrm{Co})$, iron $(\mathrm{Fe})$, aluminum (A1), indium $(\mathrm{In})$ and antimony $(\mathrm{Sb})$. The high capacity retention of the lithium-metal alloys comes at the expense of enormous volume changes leading to mechanical degradation. These large volume changes make the metal particles to coalesce reducing the available surface area and cause material cracking leading to a loss in electronic conductivity. Spinel oxide materials such as titanates can overcome these problems due to low volume expansions during intercalation process. In addition, these materials react with electrolyte during the first few cycles leading to the formation of SEI layers and hence, chemical degradation of these materials. As a result of both mechanical and chemical degradation, the available material for lithium alloying decreases and hence, shows capacity fading with cycling. In order to overcome these problems, metal oxides have been used. The overall electrochemical irreversible reaction of $\mathrm{Li}$ with metal oxide is presented below:

$$
\mathrm{MO}+2 \mathrm{Li} \rightarrow \mathrm{Li}_{2} \mathrm{O}+\mathrm{M}
$$

The reaction above proceeds by the formation of amorphous $\mathrm{Li}_{2} \mathrm{O}$ and metal. The metal in turn alloys with $\mathrm{Li}$ as indicated in Equation (2.3) to form the $\mathrm{Li}-\mathrm{M}$ alloy. However, amorphous $\mathrm{Li}_{2} \mathrm{O}$ is believed to prevent the mechanical deformation due to volume expansions to an extent improving the stability with cycling. But the metal oxide materials still undergo similar problems of material cracking upon cycling especially at high charge-discharge rates. Metal oxide thin films and micron scale particles suffer the most from the problems that have been described above.

These problems can be overcome by the reduction in the material sizes and architectures. Nanomaterials especially one-dimensional nanowires offer characteristics 
such as high surface area to volume ratio which helps in higher $\mathrm{Li}$ alloying with the material, better strain accommodation on volume expansions, better conducting pathways due to the bonding of nanowires with the current collector without any grain boundaries and faster charge transport because of low diffusion lengths inside the nanomaterials. Nanoparticles can also increase the initial capacity but suffer from conductivity problems due to the presence of grain boundaries. Similarly, 3-dimenisonal nanomaterial architectures can significantly improve the battery performance and efficiency. This section discusses the different metal oxide nanomaterial performances as electrode materials for LIBs.

\subsubsection{Tin oxide $\left(\mathrm{SnO}_{2}\right)$}

Of the various metal and metal oxide materials systems, both tin ( $\mathrm{Sn})$ and $\mathrm{SnO}_{2}$ are interesting anode materials for LIBs because of their semiconducting properties combined with high capacity compared to that of graphite. Idota et al. ${ }^{73}$ have shown that an amorphous tin-based oxide retained a capacity of $\sim 650 \mathrm{mAhg}^{-1}$ after the first cycle with low fading up to 100 cycles. Ying et al. ${ }^{74}$ has first reported the use of $\mathrm{SnO}_{2}$ nanowires as anodes for LIBs. Nanowires in this study were synthesized by thermal Sn evaporation with gold (Au) as a catalyst in a vapor-solid-liquid (VLS) mechanism ${ }^{75}$. These nanowires exhibit a high initial discharge capacity of $2133 \mathrm{mAhg}^{-1}$ at a current density of $0.5 \mathrm{mAcm}^{-2}$, which is higher than the theoretical capacities of both $\mathrm{Sn}$ and $\mathrm{SnO}_{2}$. Although the high initial capacity retention requires further analysis, it is attributed to high surface to volume ratio of the nanowires and the abundance of surface defects on the nanowire periphery which allow higher lithium incorporation into the material. 
Despite the high initial capacity, the reversible capacity drops to $\sim 700 \mathrm{mAhg}^{-1}$ at the end of 15 cycles with $3.9 \%$ capacity fade per cycle and an indication to drop further with cycling.

In another study, ${ }^{76}$ self-catalyzed $\mathrm{SnO}_{2}$ nanowires were grown without the use of a catalyst which can act as impurity and hence, cause capacity loss with cycling ${ }^{74,77}$. The metal source was a ball-milled mixture of $\mathrm{SnO}$ and $\mathrm{Sn}$ powder which on thermal evaporation deposit $\mathrm{SnO}_{2}$ nanowires on $\mathrm{Si}$ subsrates. The deposition occurs at lower temperatures than conventional VLS mechanisms. These materials show a high initial capacity $\left(2137 \mathrm{mAhg}^{-1}\right)$ with columbic efficiency of $47 \%$ which is higher than the bulk $\mathrm{SnO}_{2}$ powders ${ }^{78}$. These materials retain a capacity of $300 \mathrm{mAhg}^{-1}$ after 50 cycles which shows a lower capacity fading of $1.5 \%$ when compared to other $\mathrm{SnO}_{2}$ nanowire systems ${ }^{74} \cdot \mathrm{SnO}_{2}$ nanotubes have also exhibited a high initial capacity of $2304 \mathrm{mAhg}^{-1}$ with columbic efficiency of $39 \%$, with capacity retention of $300 \mathrm{mAhg}^{-1}$ with $1.9 \%$ capacity fade per cycle ${ }^{78}$. The basic electrochemical reaction of Li with Sn follows the proposition below: ${ }^{79}$

$$
\begin{gathered}
\mathrm{SnO}_{2}+4 \mathrm{Li}^{+}+4 e^{-} \rightarrow 2 \mathrm{Li}_{2} \mathrm{O}+\mathrm{Sn} \\
\mathrm{Sn}+x \mathrm{Li}^{+}+x e^{-} \leftrightarrow L i_{x} \mathrm{Sn}(0 \leq x \leq 4.4)
\end{gathered}
$$

The equations above indicate that $\mathrm{Li}_{2} \mathrm{O}$ is formed irreversibly during the first cycle and then, $\mathrm{Sn}$ alloys with $\mathrm{Li}$ to form a $\mathrm{Li}-\mathrm{Sn}$ alloy with up to $4.4 \mathrm{Li}$ per an atom of Sn. As indicated before, Sn undergoes a $259 \%$ volume expansion on alloying which leads to material degradation and capacity fade with cycling. 
Heterostructured coaxial $\mathrm{SnO}_{2}-\mathrm{In}_{2} \mathrm{O}_{3}$ nanowires synthesized by thermal evaporation method show 2 orders of magnitude higher conductivity than $\mathrm{SnO}_{2}$ nanowires ${ }^{80}$. These nanowires show capacity retention of $\sim 700 \mathrm{mAhg}^{-1}$ at a $\mathrm{C} / 5$ rate when cycled between $0.01 \mathrm{~V}-3 \mathrm{~V}$. $\mathrm{SnO}_{2}$ nanorods were synthesized using nonhydrothermal processes at temperatures in the range of $320-700^{\circ} \mathrm{C}^{81}$. The as-synthesized nanorods which are $15 \mathrm{~nm}$ in diameters have high aspect ratios. They were then subjected to two different potential windows at constant current density of $0.1 \mathrm{Ag}^{-1}$ which offer interesting perspectives. The first potential window of $2 \mathrm{~V}-5 \mathrm{mV}$ showed high initial capacity of $1100 \mathrm{mAhg}^{-1}$ but the capacity degraded to $400 \mathrm{mAhg}^{-1}$ at the end of 60 cycles. The material cycled between $1 \mathrm{~V}-5 \mathrm{mV}$ exhibited initial capacity of $700 \mathrm{mAhg}^{-1}$ with capacity retention of over $620 \mathrm{mAhg}^{-1}$ after $60 \mathrm{cycles}^{61}$. It was shown in this study that the nanorods on Li alloying disintegrate to form shorter rods and Sn nanoparticles which retain their physical structure leading to enhanced performance.

Spray pyrolysis technique was used to synthesize spherical porous $\mathrm{SnO}_{2}$ nanocrystals with an average diameter of $5 \mathrm{~nm}^{82}$. These nanocrystals show initial capacity retention of $601 \mathrm{mAhg}^{-1}$ with an exceptional $68 \%$ retention in capacity after 50 cycles which is $410 \mathrm{mAhg}^{-1}$. It has also been shown in the same study that increasing the particle size to $100 \mathrm{~nm}$ retains only $19 \%$ of the initial capacity after 50 cycles $^{82}$. It is however, interesting to see that $5 \mathrm{~nm} \mathrm{SnO}_{2}$ nanoparticles show exceptional cycling stability even though the inherent capacity is limited. Rutile $\mathrm{SnO}_{2}$ nanocrystals with diameters in the range of 50-100 nm were synthesized by the modified hydrothermal method $^{83}$. Tin chloride was the precursor used in the synthesis process and the concentration of hydrochloric acid controlled the size of the nanocrystals. An 
electrochemical study revealed that these nanocrystals show high first cycle discharge capacity of $1166 \mathrm{mAhg}^{-1}$ which fades to $631 \mathrm{mAhg}^{-1}$ after second cycle and retains up to $530 \mathrm{mAhg}^{-1}$ after 20 cycles at current density of $0.4 \mathrm{mAcm}^{-2}$. In these nanocrystals, better crystallinity of the material is believed to enhance lithium diffusion without obstacles leading to better capacity retention ${ }^{83}$. Hollow $\mathrm{SnO}_{2}$ nanostructures are obtained by stannate thermal treatment in a mixture of ethanol and water $^{84}$. The as-synthesized material consists of interconnected hollow particles which range between $80-750 \mathrm{~nm}$ in diameter and 10-30 $\mathrm{nm}$ shell thickness based on the precursor concentration and the additives used for the synthesis process. As seen in most of the nanoscale $\mathrm{SnO}_{2}$ materials, these hollow structures shown initial capacity of $1140 \mathrm{mAhg}^{-1}$ at $\mathrm{C} / 5$ rate and retain capacity of $\sim 470 \mathrm{mAhg}^{-1}$ after 40 cycles $^{84}$. In this study, the hollow nature of the materials accommodates the volume expansions and hence, stability is achieved.

$\mathrm{SnO}_{2}$ have also been used as composites with other materials to increase the capacity and stability. Pyrolysis technique in argon atmosphere was used for the synthesis of $\mathrm{SnO}_{2} / \mathrm{Sn}$-coated graphite composite. Sn content as determined by ICP was $0.54 \mathrm{wt} \%$ in graphite ${ }^{85}$. The material exhibits initial capacity of $272 \mathrm{mAhg}^{-1}$ when cycled between $0.01 \mathrm{~V}-2 \mathrm{~V}$ at a current density of $0.325 \mathrm{mAcm}^{-2}$. The material retains $95.8 \%$ of this capacity over 50 cycles, indicating that composites seem to relieve the stresses associated with mechanical fracture due to volume expansions improving the material properties ${ }^{85}$. Wang et al., ${ }^{86}$ have synthesized $\mathrm{SnO}_{2}$ nanotubes using alumina template with $\mathrm{SnO}_{2}$ nanoparticle loading. These nanotubes were used to form uniform CNT overlayer on their external surface leading to core-shell tubular nanostructures ${ }^{87}$. These materials exhibited $92.5 \%$ of the capacity $\left(\sim 600 \mathrm{mAhg}^{-1}\right)$ after 200 cycles, which is on par with the 
performance of commercial graphite. In another study, mesoporous $\mathrm{SnO}_{2}$ synthesized using hydrothermal method was uniformly dispersed onto MWCNTs to obtain mesoporous-tube structure ${ }^{88}$. These structures have been shown to retain $55.3 \%$ of the initial capacity, which is $344.5 \mathrm{mAhg}^{-1}$ for up to 50 cycles at a current density of 33.3 $\mathrm{mAg}^{-1}$. The enhanced performance has been attributed to one-dimensional electronic conductivity, high surface area to volume ratio and ability to relax the mechanical stress. The $\mathrm{Sn} / \mathrm{SnO}_{2}$ particle composites have been synthesized and used as anodes with capacity retention of $549 \mathrm{mAhg}^{-1}$ after 40 cycles $^{89}$. These particle composites consist of dispersed $\mathrm{Sn}$ particles in a $\mathrm{SnO}_{2}$ matrix to create good separation between the $\mathrm{Sn}$ particles. This separation prevents from Sn agglomeration to an extent and hence, helps in good capacity retention with cycling. Although the use of nanomaterials has shown improved performance over thin films of the same material as seen from the discussion above, they still do not perform up to the full potential of the materials. The work discussed above using nanoscale tin oxide-based materials including the composite materials report capacities ranging from 300 to $620 \mathrm{mAhg}^{-1}$ after 50 cycles which are below the theoretical capacities of $\mathrm{Sn}$ and $\mathrm{SnO}_{2}$. It is hence, necessary to look at other architectures including 3-dimensional architectures to improve the battery efficiency and to develop strategies which increase the stability of nanoscale metal/metal oxide systems with high capacity retention.

\subsubsection{Cobalt oxide $\left(\mathrm{CO}_{3} \mathrm{O}_{4} / \mathrm{CoO}\right)$}

$\mathrm{Co}_{3} \mathrm{O}_{4}$ is another interesting nagnetic p-type semiconductor material with theoretical capacity as high as $890 \mathrm{mAhg}^{-1}$. $\mathrm{CoO}$ and $\mathrm{Co}_{3} \mathrm{O}_{4}$ materials have exhibited 
good capacity retention properties of up to $93.4 \%$ of initial capacity until 100 cycles $^{90}$. Poizot et al., ${ }^{90}$ have shown that transition metal oxides in their nanoscale form react with lithium as follows:

$$
\begin{gathered}
\mathrm{CoO}+2 \mathrm{Li} \leftrightarrow \mathrm{Li}_{2} \mathrm{O}+\mathrm{Co} \\
\mathrm{Co}_{3} \mathrm{O}_{4}+8 \mathrm{Li} \leftrightarrow 4 \mathrm{Li}_{2} \mathrm{O}+3 \mathrm{Co}
\end{gathered}
$$

The above reactions indicate that nanoscale materials can aid in $\mathrm{Li}_{2} \mathrm{O}$ decomposition reversibly which is otherwise electrochemically inactive. This led to investigation of different nanoscale $\mathrm{Co}_{3} \mathrm{O}_{4}$ architectures as potential anodes in LIBs. Poizot et al., ${ }^{90}$ has first shown that nano-sized $\mathrm{CoO}$ and $\mathrm{Co}_{3} \mathrm{O}_{4}$ retain exceptional capacities of $700 \mathrm{mAhg}^{-1}$ and $850 \mathrm{mAhg}^{-1}$ (C/5 rate) respectively with $100 \%$ capacity retention for up to 100 cycles. These materials exhibit $>95 \%, 95 \%,>90 \%$ and $85 \%$ capacity retentions, at $0.25 \mathrm{C}, 0.5 \mathrm{C}, 1 \mathrm{C}$ and $2 \mathrm{C}$ rates respectively, indicating good rate capability. Thermal decomposition of $\mathrm{Co}\left(\mathrm{NO}_{3}\right)_{2} \cdot 6 \mathrm{H}_{2} \mathrm{O}$ precursor inside an anodic aluminum oxide membrane led to the formation of $\mathrm{Co}_{3} \mathrm{O}_{4}$ nanotubes ${ }^{91} \cdot \mathrm{Co}_{3} \mathrm{O}_{4}$ nanorods were also synthesized by precursor powder calcination ${ }^{92}$. The nanotubes show an initial capacity of $850 \mathrm{mAhg}^{-1}$ whereas the nanorods show lower capacity retention of 815 $\mathrm{mAhg}^{-1}$. Nanotubes and nanorods when cycled 100 times retain capacities of 500 and 480 $\mathrm{mAhg}^{-1}$ corresponding to $58.8 \%$ and $57.8 \%$ of initial capacity retention respectively ${ }^{91}$. Improved material characteristics are attributed to the porous nature of the nanotubes and nanorods. It has to be noted here that similar architectures show similar performance without significant changes. Porous $\mathrm{Co}_{3} \mathrm{O}_{4}$ nanotubes were synthesized by sonicating 5$10 \mathrm{~nm}$ nanoparticles in solution comprising of hexane and $\mathrm{Co}_{4}(\mathrm{CO})_{12}$ using CNT templates ${ }^{93}$. This synthesis procedure eliminates the disadvantages associated with 
alumina template use and makes pure material for high performance LIBs. The assynthesized nanotubes are $30 \mathrm{~nm}$ in diameter and show an exceptional first cycle capacity of $1918 \mathrm{mAhg}^{-1}$, maintaining a constant capacity of $1200 \mathrm{mAhg}^{-1}$ for up to 20 cycles $^{93}$. The loss in the first cycle capacity is attributed to the SEI formation and $\mathrm{Li}_{2} \mathrm{O}$ phase which could not be decomposed. Nam et al., ${ }^{94}$ have presented an interesting route for $\mathrm{Co}_{3} \mathrm{O}_{4}$ nanowire synthesis using viruses. $\mathrm{E} 4$ viruses were incubated in cobalt chloride solution and subsequent reduction in $\mathrm{NaBH}_{4}$ led to the formation of crystalline $\mathrm{Co}_{3} \mathrm{O}_{4}$ nanowires. However, introduction of gold $(\mathrm{Au})$ nanoparticles and $\mathrm{Au}$ binding peptide motif during the synthesis process can lead to the formation of $\mathrm{Co}_{3} \mathrm{O}_{4}$ nanowires with 5 $\mathrm{nm} \mathrm{Au}$ nanoparticles dispersed evenly inside them in the mass ratio of $0.976: 0.024$ respectively. Au containing nanowires show capacity retention of $>1000 \mathrm{mAhg}^{-1}$ until 8 cycles which is much improved than the capacity retention of pure nanowires which is $700 \mathrm{mAhg}^{-1}$ after 8 cycles. Au is believed to increase electronic conductivity and reversibility of $\mathrm{Li}_{2} \mathrm{O}$ and help in high capacity retention. This study demonstrates the application of biological principles to the synthesis of nanomaterials and their applications with better performance.

Mesoporous $\mathrm{Co}_{3} \mathrm{O}_{4}$ nanowire arrays were grown using a template free ammoniainduced evaporation method ${ }^{95}$. These arrays have shown to retain a capacity of $>700$ $\mathrm{mAhg}^{-1}$ up to 20 cycles at $1 \mathrm{C}$ rate. In the same study, a comparison of broken nanowires exhibits a capacity of $350 \mathrm{mAhg}^{-1}$ whereas the commercial powders retain $80 \mathrm{mAhg}^{-1}$ capacities. Further analysis has indicated that arrays retain exceptional capacities of 450 $\mathrm{mAhg}^{-1}$ at $20 \mathrm{C}\left(69 \%\right.$ capacity retention) and $240 \mathrm{mAhg}^{-1}$ at $50 \mathrm{C}(50 \%$ capacity retention ${ }^{96}$. Samples after cycling analyzed using transmission electron microscopy 
(TEM) showed highly interconnected Co metal nanoclusters inside the amorphous matrix which is predominantly $\mathrm{Li}_{2} \mathrm{O}$. In such a sample, enhanced electronic conductivity is generated due to the interconnected networks and the connectivity with the substrate. The $\mathrm{Li}^{+}$ion diffusion is also enhanced due to the nanoscale domains of Co which reduces their diffusion lengths. In addition, the presence of nanoscale Co domains also enhances the reversibility of amorphous $\mathrm{Li}_{2} \mathrm{O}$. These three factors are believed to lead the exceptional capacity retention and the rate performance of the nanowire arrays. Another recent study has shown that branched cobalt oxide nanowires have shown capacity

retention of $1043 \mathrm{mAhg}^{-1}$ for up to 50 cycles at current density of $100 \mathrm{mAg}^{-1}$. The average capacity fading for these materials is $0.15 \%$ per cycle ${ }^{97} \cdot \mathrm{Co}_{3} \mathrm{O}_{4}$ nanomaterials have shown exceptional battery characteristics in terms of reversible capacity and rate capability. In addition, modification of the material architectures has predominantly increased the battery capacity and efficiency.

\subsubsection{Molybdenum oxide $\left(\mathrm{MoO}_{3}\right)$}

$\mathrm{MoO}_{3}$ is another well-known anode material for lithium insertion. The theoretical capacity of $\mathrm{MoO}_{3}$ can be as high as $1340 \mathrm{mAhg}^{-1}$. The lithiation into $\mathrm{MoO}_{3}$ is believed to take place in two stages: Stage I corresponds up to a potential of $1.5 \mathrm{~V}$. During this stage, the $\mathrm{Li}$ intercalation with $\mathrm{MoO}_{3}$ follows the mechanism ${ }^{98}$ :

$$
\mathrm{MoO}_{3}+\mathrm{xLi}^{+}+\mathrm{xe}^{-} \leftarrow \rightarrow \mathrm{Li}_{\mathrm{x}} \mathrm{MoO}_{3}
$$

This intercalation can be further regrouped based on the amount of $\mathrm{Li}$. The initial $\mathrm{Li}$ uptake $(x<0.25)$ occurs around $2.8 \mathrm{~V}$ with the co-existence of $\mathrm{MoO}_{3}$ and $\mathrm{Li}_{\mathrm{x}} \mathrm{MoO}_{3}$. The solid solution $\mathrm{Li}_{\mathrm{x}} \mathrm{MoO}_{3}$ with $0.25<\mathrm{x}<0.5$ is formed between the potentials of $2.4-2.8 \mathrm{~V}$. 
Similarly, higher lithium content solution with $1<x<1.5$ is formed at potentials lower than $2.4 \mathrm{~V}$ up to a potential of $1.5 \mathrm{~V}$. The high lithium content is accommodated in the interlayer spacing between octahedron Mo-O layers and intralayers. Most of the Li ions intercalated at these potentials can subsequently be extracted from the material leading to reversibility of the reaction. Lithium ion intercalation during stage II corresponds to potentials below $0.5 \mathrm{~V}$ and occurs by the following mechanism ${ }^{99}$ :

$$
\mathrm{Li}_{\mathrm{x}} \mathrm{MoO}_{3}+\mathrm{yLi}^{+}+\mathrm{ye}^{-} \leftarrow \rightarrow \mathrm{Mo}+3 \mathrm{Li}_{2} \mathrm{O}
$$

In the lower voltage range, lithium reacts with the solid solution to consequently form metal and $\mathrm{Li}_{2} \mathrm{O}$. As seen with $\mathrm{Co}_{3} \mathrm{O}_{4}, \mathrm{MoO}_{3}$ also forms the metal and the metal nanoclusters aid the reversible decomposition of $\mathrm{Li}_{2} \mathrm{O}$. During the reverse process, usually $\mathrm{Li}_{2} \mathrm{O}$ undergoes partial decomposition producing amorphous $\mathrm{MoO}_{2}$ and residual $\mathrm{Li}_{2} \mathrm{O}$.

$\mathrm{MoO}_{3}$ doped with sodium $\left(\mathrm{NaMoO}_{3}\right)$ have shown high initial capacity of 940 $\mathrm{mAhg}^{-1}$ in the potential window of 3.5 to $0.005 \mathrm{~V}$ but capacity degradation occurs on cycling with $<400 \mathrm{mAhg}^{-1}$ capacity after 100 cycles $^{100}$. Hydrothermal technique was employed to synthesize $\alpha-\mathrm{MoO}_{3}$ nanobelts which were then lithiated using $\mathrm{LiCl}$ treatment ${ }^{101}$. Both pure $\alpha-\mathrm{MoO}_{3}$ nanobelts and lithiated nanobelts have been cycled between $3.7-1.5 \mathrm{~V}$. Although pure nanobelts show a higher initial capacity $\left(301 \mathrm{mAhg}^{-1}\right)$ than lithiated nanobelts $\left(240 \mathrm{mAhg}^{-1}\right)$, lithiated electrodes show better capacity retention with cycling ( 220 mAhg $\left.{ }^{-1}\right)$ compared to pure nanobelts $\left(\sim 175 \mathrm{mAhg}^{-1}\right)$. It has been shown that lithiated $\mathrm{MoO}_{3}$ nanobelts have better structural stability and show two orders of magnitude higher conductivity than pure nanobelts and hence, better performance with cycling. $\beta-\mathrm{MoO}_{3}$ nanosheets have been synthesized by microplasma processing under 
specific conditions on $\mathrm{Si}(100)$ substrate coated with $(\mathrm{Pt})$ platinum layer ${ }^{102}$. These samples showed a charge capacity of $370 \mathrm{mAhg}^{-1}$ at current density $10 \mu \mathrm{Ag}^{-1}$, higher than that reported for $\alpha-\mathrm{MoO}_{3}{ }^{103}$. Hot wire chemical vapor deposition (HWCVD) was used to synthesize crystalline molybdenum oxide nanoparticles with diameters in the range of 5$20 \mathrm{~nm}^{104}$. They have shown to retain a capacity of $630 \mathrm{mAhg}^{-1}$ for up to 150 cycles when cycled between $3.5 \mathrm{~V}$ and $0.005 \mathrm{~V}$ at a $\mathrm{C} / 10$ rate. The nanoparticles have shown a capacity retention of $\sim 500 \mathrm{mAhg}^{-1}$ at $2 \mathrm{C}$ rate whereas $5 \mu \mathrm{m}$ diameter particles show capacity degradation with cycling $\left(<300 \mathrm{mAhg}^{-1} \text { after } 25 \text { cycles }\right)^{105}$. Post lithiated sample $\mathrm{XRD}$ and in situ Raman analysis has shown that the lithiation increases the disorderliness of the nanoparticles. In addition, First-principles molecular dynamics has shown that heavy Mo-framework helps in preserving the mechanical integrity despite volume expansions during the lithiation process. Ball milled $\mathrm{MoO}_{3-\mathrm{x}}$ samples with nanocrystallites have also been shown to exhibit a high initial discharge capacity of 1100 $\mathrm{mAhg}^{-1}$ but the material undergoes capacity degradation with cycling and retains $\sim 620$ $\mathrm{mAhg}^{-1}$ after 35 cycles $^{99}$. These studies have indicated that $\mathrm{MoO}_{3}$ is a promising anode material with a scope for improving the capacity and the rate capability.

\subsubsection{Iron oxide $\left(\mathrm{Fe}_{2} \mathrm{O}_{3} / \mathrm{Fe}_{3} \mathrm{O}_{4}\right)$}

$\mathrm{Fe}_{2} \mathrm{O}_{3}$ is another interesting material which has theoretical capacities as high as $1436 \mathrm{mAhg}^{-1}$. In addition to high capacity, these materials are inexpensive and are nontoxic. The overall electrochemical reaction of $\mathrm{Fe}_{2} \mathrm{O}_{3}$ with $\mathrm{Li}$ is as follows ${ }^{106}$ :

$$
\mathrm{Fe}_{2} \mathrm{O}_{3}+6 \mathrm{Li} \leftarrow \rightarrow \mathrm{Fe}+3 \mathrm{Li}_{2} \mathrm{O}
$$


specific conditions on $\mathrm{Si}(100)$ substrate coated with $(\mathrm{Pt})$ platinum layer ${ }^{102}$. These samples showed a charge capacity of $370 \mathrm{mAhg}^{-1}$ at current density $10 \mu \mathrm{Ag}^{-1}$, higher than that reported for $\alpha-\mathrm{MoO}_{3}{ }^{103}$. Hot wire chemical vapor deposition (HWCVD) was used to synthesize crystalline molybdenum oxide nanoparticles with diameters in the range of 5$20 \mathrm{~nm}^{104}$. They have shown to retain a capacity of $630 \mathrm{mAhg}^{-1}$ for up to 150 cycles when cycled between $3.5 \mathrm{~V}$ and $0.005 \mathrm{~V}$ at a $\mathrm{C} / 10$ rate. The nanoparticles have shown a capacity retention of $\sim 500 \mathrm{mAhg}^{-1}$ at $2 \mathrm{C}$ rate whereas $5 \mu \mathrm{m}$ diameter particles show capacity degradation with cycling $\left(<300 \mathrm{mAhg}^{-1} \text { after } 25 \text { cycles }\right)^{105}$. Post lithiated sample XRD and in situ Raman analysis has shown that the lithiation increases the disorderliness of the nanoparticles. In addition, First-principles molecular dynamics has shown that heavy Mo-framework helps in preserving the mechanical integrity despite volume expansions during the lithiation process. Ball milled $\mathrm{MoO}_{3-\mathrm{x}}$ samples with nanocrystallites have also been shown to exhibit a high initial discharge capacity of 1100 $\mathrm{mAhg}^{-1}$ but the material undergoes capacity degradation with cycling and retains $\sim 620$ $\mathrm{mAhg}^{-1}$ after 35 cycles $^{99}$. These studies have indicated that $\mathrm{MoO}_{3}$ is a promising anode material with a scope for improving the capacity and the rate capability.

\subsubsection{Iron oxide $\left(\mathrm{Fe}_{2} \mathrm{O}_{3} / \mathrm{Fe}_{3} \mathrm{O}_{4}\right)$}

$\mathrm{Fe}_{2} \mathrm{O}_{3}$ is another interesting material which has theoretical capacities as high as $1436 \mathrm{mAhg}^{-1}$. In addition to high capacity, these materials are inexpensive and are nontoxic. The overall electrochemical reaction of $\mathrm{Fe}_{2} \mathrm{O}_{3}$ with $\mathrm{Li}$ is as follows ${ }^{106}$ :

$$
\mathrm{Fe}_{2} \mathrm{O}_{3}+6 \mathrm{Li} \leftrightarrow \rightarrow \mathrm{Fe}+3 \mathrm{Li}_{2} \mathrm{O}
$$


In this reaction, the complete extraction of $\mathrm{Li}$ from $\mathrm{Li}_{2} \mathrm{O}$ is not possible and hence, there is a capacity loss during cycling at least for the first few cycles. $\alpha-\mathrm{Fe}_{2} \mathrm{O}_{3}$ nanotubes synthesized by a templating technique showed a first discharge capacity of $1415 \mathrm{mAhg}^{-1}$, close to the theoretical capacity ${ }^{106}$. TEM image after characterization indicated agglomeration of fine particles on the surface indicating high activity on the surface. In another study, $\alpha-\mathrm{Fe}_{2} \mathrm{O}_{3}$ nanoflakes were synthesized by thermal treatment on a copper substrate. These nanoflakes exhibit a capacity of $\sim 680 \mathrm{mAhg}^{-1}$ for up to 80 cycles at a $0.1 \mathrm{C}$ rate. The nanoflakes also exhibit $>98 \%$ columbic efficiency after the $15^{\text {th }}$ cycle ${ }^{107}$. Taberna et al., ${ }^{108}$ have shown that $\mathrm{Fe}_{3} \mathrm{O}_{4} / \mathrm{Cu}$ nano architectures show exceptional capacity retention. The electrodes were deposited using a two step process. Initially, $\mathrm{Cu}$ nanorods are deposited onto a copper foil using electrodeposition. Subsequently, $\mathrm{Fe}_{3} \mathrm{O}_{4}$ is electrodeposited onto the copper nanorods to obtain core-shell architecture. They show excellent capacity retention of $\sim 900 \mathrm{mAhg}^{-1}$ at $\mathrm{C} / 32$ rate with $100 \%$ capacity retention with cycling for up to 50 cycles $^{108}$. These nano-architectures exhibit capacity retention of $80 \%$ at $8 \mathrm{C}$ rate, which is less than 7 minutes. The electrodes cycled at $\mathrm{C} / 2$ rate characterized by SEM show no morphology changes after 100 cycles indicating a robust mechanical electrode. $\mathrm{Fe}_{2} \mathrm{O}_{3}$ have thus proved to be promising materials with high rate capabilities but they still require significant improvements in the rates and the inexpensive manufacturing of these materials. 


\subsubsection{Titania $\left(\mathrm{TiO}_{2}\right)$}

$\mathrm{TiO}_{2}$ is another well-known anode material for lithium insertion. Although the theoretical capacity of $\mathrm{TiO}_{2}$ is low, it exhibits excellent rate capability, is abundant and non-toxic which makes it quite suitable for LIBs. The lithiation mechanism is as follows:

$$
\mathrm{TiO}_{2}+\mathrm{xLi}^{+}+\mathrm{xe}^{-} \leftarrow \rightarrow \mathrm{Li}_{\mathrm{x}} \mathrm{TiO}_{2}
$$

The amount of $\mathrm{Li}$ intercalated into the material depends on the phase of the material. Brookite titania (orthorhombic structure) was synthesized by a reaction between titanium trichloride and urea ${ }^{109}$. The synthesized nanocrystallites are $10-20 \mathrm{~nm}$ in size. MWCNTs were mixed with nanocrystallites to obtain nanocomposites which retains a capacity of $160 \mathrm{mAhg}^{-1}$ after 50 cycles (Theoretical capacity: $165 \mathrm{mAhg}^{-1}$ ). $\mathrm{TiO}_{2}$-B nanowires were synthesized using hydrothermal technique by reacting sodium hydroxide and anatase $\mathrm{TiO}_{2}{ }^{110}$. These nanowires show capacity retention of $\sim 190 \mathrm{mAhg}^{-1}$ after 100 cycles at $50 \mathrm{mAg}^{-1}$. They retain a capacity of $85 \mathrm{mAhg}^{-1}$ at $3000 \mathrm{mAg}^{-1}$ (35C) rate. In another study, carbon black coated $\mathrm{TiO}_{2}$ nanotube arrays exhibited stable capacity retention with cycling at high charge-discharge rates which is attributed to faster $\mathrm{Li}$ ion diffusion through titania and enhanced electronic conduction through the carbon black ${ }^{111}$. Alumina templates were used to synthesize $\mathrm{TiO}_{2}$ nanotubes with mesoporous wall structure. These nanotubes retain capacities of 162,120 and $105 \mathrm{mAhg}^{-1}$ at current densities of 1,10 and $40 \mathrm{Ag}^{-1}$ (about $240 \mathrm{C}$ ) respectively after 100 cycles $^{112}$. Exceptional rate capability is due to the efficient transport of lithium ions and electrons through the mesopore channels and 3D network architecture respectively. $\mathrm{TiO}_{2}$ is a promising high rate capable material with exceptional properties and is already, a frontrunner as high rate anode for commercial LIBs. 


\subsubsection{Other metal oxides as anodes for LIBs}

In addition to the high capacity metal oxide materials discussed above, other oxides have been studied for their electrochemical properties. For example, nickel oxide (NiO) nanoshafts were synthesized by a scalable process of chemical precipitation followed by thermal decomposition using $\mathrm{Ni}(\mathrm{OH})_{2}$ precursors ${ }^{113}$. Electrochemical testing indicated high initial capacity of $\sim 870 \mathrm{mAhg}^{-1}$ but the capacity fades to $>400 \mathrm{mAhg}^{-1}$ by the $30^{\text {th }}$ cycle. However, this is a significant improvement over nanoparticles which retain $>200 \mathrm{mAhg}^{-1}$ after 30 cycles. These materials require rigorous study before labeling them as practical anodes. Manganese oxide $\left(\mathrm{MnO}_{2}\right)$ was also used as anode materials. Interconnected $\mathrm{MnO}_{2}$ nanowires were synthesized in the diameter ranges of 8-18 nm depending on the synthesis temperature. Nanowires synthesized at a temperature of $500^{\circ} \mathrm{C}$ with diameters $\sim 8 \mathrm{~nm}$ showed capacity retention of $800 \mathrm{mAhg}^{-1}$ after 100 cycles when cycled between $2-0.01 \mathrm{~V}$ with low capacity fade ${ }^{114}$. All these studies indicate a considerable effort in studying metal oxide nanomaterials as potential anode materials. A collection of the results using metal oxide nanowires are presented in Table 2.3. Data presented in the table indicate the better performance of nanomaterials over their micronscale/thin film counterparts in terms of both higher reversible capacity and stability with cycling. Although these efforts also present promising results for nanomaterials going into the future, rate capability, safety and high capacity are some of the issues with LIBs that need to be addressed. In this conext, new and innovative 3-dimensional architectures based on nanowires/nanotubes are necessary to further decrease $\mathrm{Li}$ ion diffusion lengths and improve the performance of LIBs. Developing these architectures requires a lot of materials engineering in a way best suited to improve the battery efficiency. 
Table 2.3: Summary of retained capacities of metal oxide nanomaterials.

\begin{tabular}{|c|c|c|c|c|}
\hline $\begin{array}{l}\text { Anode } \\
\text { material }\end{array}$ & Material structure & $\begin{array}{l}\text { Capacity } \\
\text { retention, } \\
\text { mAhg }^{-1}\end{array}$ & $\begin{array}{l}\text { No. of } \\
\text { Cycles }\end{array}$ & $\begin{array}{c}\text { Post-lithiated sample } \\
\text { structure }\end{array}$ \\
\hline \multirow{10}{*}{$\begin{array}{l}\text { Cobalt oxide } \\
\qquad\left(\mathrm{Co}_{3} \mathrm{O}_{4}\right)\end{array}$} & Nanoparticles ${ }^{90}$ & 700 & 100 & \multirow{2}{*}{$\begin{array}{c}\text { Smaller nanoparticles } \\
\text { which are well dispersed }\end{array}$} \\
\hline & $\begin{array}{c}\mathrm{CoO} \\
\text { nanoparticles }^{90}\end{array}$ & $<800$ & 100 & \\
\hline & Nanotubes $^{91}$ & 520 & 100 & \multirow{3}{*}{$\begin{array}{c}\text { Co phase presence which } \\
\text { helps in the reversibility } \\
\text { of } \mathrm{Li}_{2} \mathrm{O} \text {. }\end{array}$} \\
\hline & Nanowires $^{91}$ & 500 & 100 & \\
\hline & Nanoparticles $^{91}$ & 475 & 100 & \\
\hline & Nanowires $^{94}$ & 700 & 10 & \multirow{2}{*}{$\begin{array}{c}\text { No post-lithiation } \\
\text { analysis of physical } \\
\text { structure }\end{array}$} \\
\hline & $\begin{array}{c}\text { Nanowires with } \\
\text { gold dispersed in } \\
\text { them }^{94}\end{array}$ & 1000 & 10 & \\
\hline & Broken nanowires ${ }^{96}$ & $<400$ & 20 & \multirow{3}{*}{$\begin{array}{l}\text { Arrays show stable } \\
\text { structure after } 20 \text { cycles }\end{array}$} \\
\hline & Powders $^{96}$ & $>100$ & 20 & \\
\hline & Nanowire arrays $^{96}$ & $>700$ & 20 & \\
\hline \multirow{8}{*}{$\begin{array}{l}\text { Tin oxide } \\
\left(\mathrm{SnO}_{2}\right)\end{array}$} & Nanowires $^{76}$ & $>300$ & 50 & \multirow{3}{*}{$\begin{array}{l}\text { No post-lithiation } \\
\text { analysis of physical } \\
\text { structure }\end{array}$} \\
\hline & Nanotubes $^{78}$ & $<300$ & 50 & \\
\hline & Nanoparticles $^{78}$ & $<150$ & 50 & \\
\hline & $\begin{array}{l}\text { Nanorods } \\
(1-5 \mathrm{mV}) \\
\end{array}$ & 700 & 60 & \multirow{2}{*}{$\begin{array}{l}\text { Disintegration into } \\
\text { smaller nanorods and } \\
\text { nanoparticles }\end{array}$} \\
\hline & $\begin{array}{l}\text { Nanorods } \\
(2-5 \mathrm{mV}) \\
\end{array}$ & 400 & 60 & \\
\hline & $\begin{array}{c}\text { Hollow } \\
\text { nanostructures } \\
\end{array}$ & $<500$ & 40 & $\begin{array}{l}\text { No post-lithiation } \\
\text { analysis of physical } \\
\text { structure }\end{array}$ \\
\hline & Nanowires ${ }^{80}$ & 400 & 10 & \multirow{2}{*}{$\begin{array}{c}\text { No post-lithiation } \\
\text { analysis of physical } \\
\text { structure }\end{array}$} \\
\hline & & $>700$ & 10 & \\
\hline
\end{tabular}




\begin{tabular}{|c|c|c|c|c|}
\hline & $\begin{array}{c}\mathrm{In}_{2} \mathrm{O}_{3} / \mathrm{SnO}_{2} \\
\text { heterostructured } \\
\text { nanowires }\end{array}$ & & & \\
\hline \multirow{4}{*}{$\begin{array}{l}\text { Molybdenum } \\
\text { oxide }\left(\mathrm{MoO}_{3}\right)\end{array}$} & Nanobelts ${ }^{101}$ & $<175$ & 15 & \multirow{2}{*}{$\begin{array}{l}\text { No post-lithiation } \\
\text { analysis of physical } \\
\text { structure }\end{array}$} \\
\hline & $\begin{array}{c}\text { Lithiated } \\
\text { nanobelts }\end{array}$ & $\sim 225$ & 15 & \\
\hline & Nanoparticles ${ }^{104}$ & 630 & 150 & \multirow{2}{*}{$\begin{array}{l}\text { No post-lithiation } \\
\text { analysis of physical } \\
\text { structure }\end{array}$} \\
\hline & $\begin{array}{l}\text { Ball-milled } \\
\text { crystallites }\end{array}$ & $\sim 620$ & 35 & \\
\hline \multirow{3}{*}{$\begin{array}{c}\text { Iron oxide } \\
\left(\mathrm{Fe}_{2} \mathrm{O}_{3} / \mathrm{Fe}_{3} \mathrm{O}_{4}\right)\end{array}$} & $\begin{array}{c}\mathrm{Fe}_{3} \mathrm{O}_{4} / \mathrm{Cu} \\
\text { nanoarchitectures }\end{array}$ & $<900$ & 50 & $\begin{array}{l}\text { Stable mechanical } \\
\text { structure after lithiation }\end{array}$ \\
\hline & Nanoflakes ${ }^{107}$ & $\sim 680$ & 80 & $\begin{array}{l}\text { No post-lithiation } \\
\text { analysis of physical } \\
\text { structure }\end{array}$ \\
\hline & Nanotubes ${ }^{106}$ & 1415 & 1 & $\begin{array}{l}\text { No post-lithiation } \\
\text { analysis of physical } \\
\text { structure }\end{array}$ \\
\hline \multirow{3}{*}{ Titania $\left(\mathrm{TiO}_{2}\right)$} & Nanocrystallites ${ }^{109}$ & 160 & 50 & $\begin{array}{l}\text { No post-lithiation } \\
\text { analysis of physical } \\
\text { structure }\end{array}$ \\
\hline & Nanowires ${ }^{110}$ & 190 & 100 & $\begin{array}{l}\text { No post-lithiation } \\
\text { analysis of physical } \\
\text { structure }\end{array}$ \\
\hline & Nanotubes ${ }^{112}$ & 162 & 100 & $\begin{array}{l}\text { No post-lithiation } \\
\text { analysis of physical } \\
\text { structure }\end{array}$ \\
\hline $\begin{array}{l}\text { Nickel oxide } \\
\quad(\mathrm{NiO})\end{array}$ & Nanoshafts ${ }^{113}$ & $>400$ & 30 & $\begin{array}{l}\text { No post-lithiation } \\
\text { analysis of physical } \\
\text { structure }\end{array}$ \\
\hline \multirow{3}{*}{$\begin{array}{c}\text { Manganese } \\
\text { oxide }\left(\mathrm{MnO}_{2}\right)\end{array}$} & $\begin{array}{l}\text { Nanowires } \\
\left(100^{\circ} \mathrm{C}\right)^{114}\end{array}$ & $<100$ & 100 & \multirow{3}{*}{$\begin{array}{l}\text { No post-lithiation } \\
\text { analysis of physical } \\
\text { structure }\end{array}$} \\
\hline & $\begin{array}{l}\text { Nanowires } \\
\left(300^{\circ} \mathrm{C}\right)^{114}\end{array}$ & $>500$ & 100 & \\
\hline & $\begin{array}{l}\text { Nanowires } \\
\left(500^{\circ} \mathrm{C}\right)^{114}\end{array}$ & 800 & 100 & \\
\hline
\end{tabular}




\subsection{Inorganic material anodes}

Apart from the metal oxide materials which have shown promise with high capacity and limited rate capability, inorganic materials especially metal nanostructures can also significantly improve LIB performance. Among these materials, Si and Ge show the most promise because of their high theoretical capacities of $4200 \mathrm{mAhg}^{-1}$ and 1600 $\mathrm{mAhg}^{-1}$ respectively. In addition, metals such as $\mathrm{Sn}, \mathrm{Ni}$, composites (alloys) of metals such as $\mathrm{CuSn}, \mathrm{SnNi}$ were studied for their electrochemical properties.

\subsubsection{Silicon (Si) nanomaterials}

Although Si has exceptional capacity, it undergoes $400 \%$ volume expansion with Li alloying and de-alloying which causes mechanical fatigue. Si alloying mechanism with Li can be best described as follows:

$$
S i+x L i^{+}+x e^{-} \leftrightarrow L i_{x} S i(0 \leq x \leq 4.4)
$$

$\mathrm{Si}$ alloys with $\mathrm{Li}$ to form different $\mathrm{Li}-\mathrm{Si}$ alloys with up to a maximum of $4.4 \mathrm{Li}^{+}$ ions per an atom of $\mathrm{Si}$. Si reactivity with $\mathrm{Li}$ is particularly, limited to high temperatures. However, it has been shown that nanocrystalline $\mathrm{Si}$ enhances the reactivity at temperatures as low as room temperature. In addition, Si exhibits low alloying potentials w.r.t $\mathrm{Li}^{+} / \mathrm{Li}$ which makes them attractive as anodes in LIBs. Hence, a number of studies have explored the use of Si in nanoscale form for the capacity improvement in a battery. Nest-like Si nanospheres were synthesized using a solvothermal technique in which the size and morphology of the spheres strongly depends on the synthesis conditions ${ }^{115}$. These nanospheres had diameters in the range of $90-110 \mathrm{~nm}$ with surface areas as high as 
$386 \mathrm{~m}^{2} \mathrm{~g}^{-1}$. The surfaces of the spheres had 5-10 nm diameter nanowires. They exhibited capacities of 3628,3291 and $3052 \mathrm{mAhg}^{-1}$ at current densities of 400,800 and 2000 $\mathrm{mAg}^{-1}$ respectively ${ }^{116}$. The material was cycled for 50 cycles at high density of 2000 $\mathrm{mAg}^{-1}(0.5 \mathrm{C})$ which showed capacity retention of $1095 \mathrm{mAhg}^{-1}$ indicating the excellent rate capability of the material ${ }^{116}$.

Decomposition of silicon precursors in AAO templates has led to the formation of silicon nanotubes ${ }^{117}$. Si nanotubes were $200-250 \mathrm{~nm}$ in diameter with an outer wall thickness of $\sim 40 \mathrm{~nm}$. They exhibit first cycle discharge and charge capacities of 3648 $\mathrm{mAhg}^{-1}$ and $3247 \mathrm{mAhg}^{-1}$ respectively, which accounts to $89 \%$ columbic efficiency and is outstanding. This is attributed to the thin film of carbon on the nanotube which prevents electrolyte decomposition and SEI formation to an extent. Capacity retentions of $>3000 \mathrm{mAhg}^{-1}$ for 200 cycles at both $3 \mathrm{C}$ and $5 \mathrm{C}$ rates were observed in a battery made of $\mathrm{LiCoO}_{2}$ cathode and $\mathrm{Si}$ nanotube anode. Post-lithiation characterization revealed that the nanotube architecture is intact even though the wall thickness increased from $\sim 40 \mathrm{~nm}$ to $\sim 300 \mathrm{~nm}$ explaining the high capacity and rate capability. Chan et al., ${ }^{118}$ have shown that Si nanowire arrays grown on steel substrates exhibit high initial capacity of $4277 \mathrm{mAhg}^{-1}$ at $\mathrm{C} / 20$ rate. Further cycling showed that the capacity remained constant at $\sim 3200 \mathrm{mAhg}^{-}$ ${ }^{1}$ for the next 10 cycles. The material was cycled at various charge-discharge rates. The material retained a capacity $>2100 \mathrm{mAhg}^{-1}$ at rate of $1 \mathrm{C}$. In addition, the material retained capacities $\sim 3500 \mathrm{mAhg}^{-1}$ for 20 cycles at $\mathrm{C} / 5$ rate indicating good rate capability. The high rate capability is attributed to the efficient electron transport as a result of the direct contact between the nanowires and the underlying substrate. Post-lithiated sample analysis has shown that the nanowires widen and increase in length due to the $400 \%$ 
volume expansion on $\mathrm{Li}$ alloying and de-alloying instead of undergoing mechanical fracture and disintegration. The nanowires remain intact and well connected to the substrate. Similarly, core-shell architectures with crystalline Si core and amorphous $\mathrm{Si}$ shell have been synthesized directly on stainless steel substrates using a one-step process $^{119}$. These nanowires retain capacity as high as $1000 \mathrm{mAhg}^{-1}$ at $0.2 \mathrm{C}$ current density for 100 cycles. They exhibit capacity retention of $\sim 800 \mathrm{mAhg}^{-1}$ at $1.6 \mathrm{C}$ for up to 100 cycles with columbic efficiency of greater than $95 \%$ after the first few cycles. The material is shown to retain the architecture after 100 cycles. The studies above indicate $\mathrm{Si}$ capability and its future as the anode material for LIBs. Nevertheless, the results presented here are preliminary and need further evaluation to understand the electrochemical aspects of nanowire material systems to be available for commercial use.

\subsubsection{Germanium (Ge) nanomaterials}

Along with its high capacity $\left(1600 \mathrm{mAhg}^{-1}\right)$, Ge has a room temperature diffusivity which is 400 times higher than that of silicon. This makes it attractive, particularly for high power (high rate) applications. In addition, Ge exhibits low alloying potentials like $\mathrm{Si}$ w.r.t $\mathrm{Li}^{+} / \mathrm{Li}$ making it suitable for practical purposes. Ge nanowire arrays were synthesized directly on metallic substrates using VLS growth mechanism ${ }^{120}$. These arrays exhibited initial capacity of $1141 \mathrm{mAhg}^{-1}$ and stable capacity of $\sim 1000$ $\mathrm{mAhg}^{-1}$ after 20 cycles at a rate of $\mathrm{C} / 20$. Nanowire arrays were subjected to rates of $\mathrm{C} / 5$, $\mathrm{C} / 2, \mathrm{C}$ and $2 \mathrm{C}$ with capacity retentions of $\sim 775, \sim 625, \sim 600, \sim 590 \mathrm{mAhg}^{-1}$ respectively, which indicate excellent diffusion properties of $\mathrm{Li}$ inside Ge. Post-lithiation characterization has shown that the Ge nanowires were amorphous but the morphology is 
intact without any structural deformations. The nanowires were well connected to the substrate and the strain due to Li alloying is accommodated by the linear and radial expansion of the Ge nanowires thus, increasing the stability with cycling. Ge nanoparticles with diameters in the range of $8-100 \mathrm{~nm}$ confined in an amorphous carbon matrix were synthesized by simple annealing process ${ }^{121}$. Ge nanoparticle diameters vary based on the synthesis temperature. Ge nanoparticles synthesized at $600^{\circ} \mathrm{C}$ showed an initial capacity of $1067 \mathrm{mAhg}^{-1}$ with $88 \%$ capacity retention after 30 cycles. The ones synthesized at $800^{\circ} \mathrm{C}$ showed initial capacity of $1748 \mathrm{mAhg}^{-1}$ but the capacity fades to $729 \mathrm{mAhg}^{-1}$ after 30 cycles. The material with the best properties has been shown to retain their mechanical integrity whereas the other samples show disintegration on cycling and hence, a loss in capacity. Ge nanostructures have shown high capacity and high rate capability which make them promising anodes for LIBs.

\subsubsection{Alloy composites}

In addition to the pure inorganic materials, intermetallic alloys have also been studied. $\mathrm{Sn}_{78} \mathrm{Ge}_{22}$ clusters have been thermally annealed at $600^{\circ} \mathrm{C}$ in vacuum to obtain $\mathrm{Sn}_{78} \mathrm{Ge}_{22} @$ carbon nanowires in core-shell architecture ${ }^{122}$. These nanowires exhibit charge capacity retention of $1107,1054,1030,1000$, and $973 \mathrm{mAhg}^{-1}$ at rates of $0.3 \mathrm{C}, 1 \mathrm{C}, 3 \mathrm{C}$, 6C and $8 \mathrm{C}$ respectively, when fabricated as coin cells. In addition, the material retains charge capacity of $>950 \mathrm{mAhg}^{-1}$ at $0.3 \mathrm{C}$ rate for up to 45 cycles which is $94 \%$ of initial capacity. The improved performance of the material has been attributed to the increased surface area and shorter Li diffusion lengths because of smaller wire diameters. Postlithiated samples show stacking faults and microtwins parallel to the nanowire axis but 
retain the nanowire architecture. The post-lithiated nanowires do not show $\mathrm{Sn}$ or Ge agglomeration. $\mathrm{Ni}_{3} \mathrm{Sn}_{4}$ intermetallic material has an associated theoretical capacity of 993 mAhg ${ }^{-1}$. In the initial attempts, electrodeposited $\mathrm{Ni}_{3} \mathrm{Sn}_{4}$ exhibit capacity retention of 300 $\mathrm{mAhg}^{-1}$ for up to 70 cycles at $1 \mathrm{C}$ rate ${ }^{123}$. However, higher rates are required for applications such as HEVs and PHEVs. In another study, $\mathrm{Cu}$ nanorods were deposited onto $\mathrm{Cu}$ foil by electrodeposition. $\mathrm{Ni}_{3} \mathrm{Sn}_{4}$ particles were deposited onto the $\mathrm{Cu}$ nanorods to obtain core-shell architectures directly on conducting $\mathrm{Cu}$ substrates. These electrodes show capacity retention of $\sim 500 \mathrm{mAhg}^{-1}$ at $0.8 \mathrm{C}$ rate with no capacity fade for up to 200 cycles. They also show stable capacity retention at various rates, with $\sim 200 \mathrm{mAhg}^{-1}$ at $10 \mathrm{C}$ rate when cycled between $1.5-0.02 \mathrm{~V}^{124}$.

\subsection{Three-dimensional nanostructured materials}

Bulk materials show high initial capacities but undergo capacity fade with cycling because of material pulverization. Nanowires have shown improved capacity retention with mechanism to accommodate the volume expansions. However, nanowires have limited charge transport capabilities in the lithiated state which in turn hinders the performance at high charge-discharge rates. In addition, the amount of active material per unit volume in these cases is a major drawback in their application as practical anodes. 3dimensional architectures with composite materials can increase the active material and improve rate characteristics for high performance batteries. 3-dimensional interconnected Titanium silicide $\left(\mathrm{TiSi}_{2}\right.$ ) nanonets have been synthesized by CVD using $\mathrm{TiCl}_{4}$ and $\mathrm{SiH}_{4}$

as the precursors directly on a Ti foil for enhanced conductivity ${ }^{125}$. Si nanoparticles were then grown directly on $\mathrm{TiSi}_{2}$ to obtain the composite 3-dimensional architecture in which 
$\mathrm{Si}$ alloys with $\mathrm{Li}$ for the high capacity whereas the $\mathrm{TiSi}_{2}$ underneath acts as the charge transport medium thus giving rise to high rate capability. 3-dimensional architectures exhibited high initial capacity of $1990 \mathrm{mAhg}^{-1}$ with capacity fade for the first 10 cycles after which the capacity remains fairly constant until the $100^{\text {th }}$ cycle to retain a capacity of $937 \mathrm{mAhg}^{-1}$ at current density of $8400 \mathrm{mAg}^{-1}$. The capacity fade occurs at a rate of $0.1 \%$ per cycle with a total capacity fade of $8.7 \%$ from the $23^{\text {rd }}$ cycle to the $100^{\text {th }}$ cycle ${ }^{125}$. Kim et al., ${ }^{126}$ have reported that thermal annealing and etching of $\mathrm{Si}$ gel and $\mathrm{SiO}_{2}$ nanoparticle composites in argon (Ar) atmosphere led to the formation of 3D porous interconnected Si particles. The pore walls are $40 \mathrm{~nm}$ thick and the pores are $200 \mathrm{~nm}$ in diameter. This material shows capacity retention of $\sim 2800$ and $\sim 2450 \mathrm{mAhg}^{-1}$ at rates of $0.2 \mathrm{C}$ and $1 \mathrm{C}$ respectively for up to 100 cycles. The capacity retentions at rates of $0.1 \mathrm{C}$ and $1 \mathrm{C}$ are $99 \%$ and $90 \%$ respectively after 100 cycles.

3D integrated all solid-sate batteries have been an idea to improve the battery performance which involves polymer electrolytes in which an anode is polymer coated with a cathode coating on the top. A report on $\mathrm{Si}$ electrode covered with $\mathrm{Li}_{3} \mathrm{PO}_{4}$ electrolyte and $1 \mu \mathrm{m}$ thick lithium cobalt oxide $\left(\mathrm{LiCoO}_{2}\right)$ cathode with another coating of the current collector exhibited reversible capacity retention of $\sim 3500 \mathrm{mAhg}^{-1}$ for up to 60 cycles at a $1 \mathrm{C}$ rate ${ }^{127}$. For comparison purposes, a conventional Si electrode in an organic $\mathrm{Li}$ ion battery electrolyte exhibited capacity of $>1000 \mathrm{mAhg}^{-1}$ after 60 cycles. Although the capacity is higher than graphite, there is a large capacity fade after the $30^{\text {th }}$ cycle until the $60^{\text {th }}$ cycle. Electrochemical deposition was used to synthesize 3D foam structured $\mathrm{Cu}_{6} \mathrm{Sn}_{5}$ alloys ${ }^{128}$. The foam walls consist of small particles of the alloy which are $\sim 350$ $\mathrm{nm}$ in diameter. These alloys exhibited capacity retention of $400 \mathrm{mAhg}^{-1}$ after 30 cycles 
at current density of $0.5 \mathrm{mAcm}^{-2}(\sim 1 \mathrm{C}$ rate). The materials have also been shown to retain $50 \%$ of this capacity at a rate of $20 \mathrm{C}$ showing marked improvement in rate capability. An optimized design of introducing dual functional CNTs into $\mathrm{CuO}$ nanomicrospheres to obtain 3D CuO-CNT composites was developed ${ }^{129}$. The chemical composition analysis has shown that the composite is made of $94 \mathrm{wt} \% \mathrm{CuO}$ and the remaining is CNTs. They exhibit an initial discharge capacity of $860 \mathrm{mAhg}^{-1}$ with constant capacity retention of $\sim 500 \mathrm{mAhg}^{-1}$ until 25 cycles at $\mathrm{C} / 10$ rate with $100 \%$ capacity retention after the second cycle. The material has capacity retention of $\sim 150 \mathrm{mAhg}^{-1}$ at $5 \mathrm{C}$ rate.

Ortiz et al., ${ }^{130}$ have reported the synthesis of nanoarchitectured composites with $\mathrm{Sn}$ and $\mathrm{SnO}_{2}$ nanowires grown on $\mathrm{TiO}_{2}$ nanotubes. The material exhibited initial capacity of $140 \mu \mathrm{Ahcm}^{-2}$ with columbic efficiency of $85 \%$ until 50 cycles at constant current density of $50 \mu \mathrm{Acm}^{-2}$ (2C). Similarly, the material exhibited capacity of $\sim 70 \mu \mathrm{Ahcm}^{-2}$ at current density of $100 \mu \mathrm{Acm}^{-2}(4 \mathrm{C})$ when cycled between $1.2-0.01 \mathrm{~V}$. A hybrid material was developed by integrating the $\mathrm{CNT}$ cathode and $\mathrm{TiO}_{2}$ nanowire anode ${ }^{131}$. They exhibited an energy density of $12.5 \mathrm{Whkg}^{-1}$ at $10 \mathrm{C}$ rate indicating constant energy density with cycling after 600 cycles. A 3D composite electrode consisted of $\mathrm{Li}_{2} \mathrm{O}-\mathrm{CuO}-\mathrm{SnO}_{2}$ using the electrostatic spray deposition technique ${ }^{132}$. The material has a multideck-cage structure with wall thickness of $60-100 \mathrm{~nm}$. The pore diameter ranges from $200 \mathrm{~nm}-1$ $\mu \mathrm{m}$. The composite electrode exhibits an initial capacity of $1174 \mathrm{mAhg}^{-1}$ with a first cycle capacity loss of $18 \%$, which is significantly low. The electrode retains capacity of $1158.5 \mathrm{mAhg}^{-1}$ after 100 cycles at $0.5 \mathrm{C}$ rate. The material retains capacities of $\sim 900$, $\sim 700$ and $<500 \mathrm{mAhg}^{-1}$ at rates of $1 \mathrm{C}, 3 \mathrm{C}$ and $8 \mathrm{C}$ respectively thus exhibiting high rate capability. 3D architectures have exhibited significant rate capability and capacity with 
cycling and hence, show promise as the future architectures for LIBs. However, the materials studied above require further improvements by a combination of materials to form hybrid architectures incorporating unique characteristics for each material leading to significant advancements in battery technology.

\subsection{Summary}

Most of the anode materials suffer from large volume expansions on lithium alloying leading to mechanical degradation of the material. In addition, SEI formation leads to chemical degradation. Hence, the capacity retention and the stability vary with the material architecture. MWCNTs have shown to retain initial capacities as high as $\sim 950 \mathrm{mAhg}^{-1}$ and 50 cycle capacity in excess of $400 \mathrm{mAhg}^{-1}$. SWCNTs have shown higher capacity retention due to higher accessible surface area for Li intercalation. Ball milled SWCNTs show high initial capacity retention with a reversible capacity of $\sim 780$ $\mathrm{mAhg}^{-1}$ with low capacity loss. Other forms of carbon including CNFs show capacities of $\sim 800 \mathrm{mAhg}^{-1}$ at low rates and capacities of $\sim 450 \mathrm{mAhg}^{-1}$ at rates as high as $0.5 \mathrm{C}$. Graphitized carbon nanobeads show capacity retentions of $\sim 180 \mathrm{mAhg}^{-1}$ at high rates of 12C. In the case of non-graphitic carbons, ordered mesoporous material shows capacity retentions of $\sim 850-900 \mathrm{mAhg}^{-1}$ until 20 cycles. Carbon based materials have exhibited good capacity retention with cycling at low to moderate charge-discharge rates.

Among the metal oxides, nanoscale tin oxide materials exhibit capacities in the range of $300-620 \mathrm{mAhg}^{-1}$ until 100 cycles with room for further improvement on exploration of new material architectures. Similarly, $\mathrm{Co}_{3} \mathrm{O}_{4}$ nanowire arrays exhibited

capacities of $>700,450,240 \mathrm{mAhg}^{-1}$ at rates of $1 \mathrm{C}, 20 \mathrm{C}$ and $50 \mathrm{C}$ respectively. Thus, 
these materials show excellent rate capability with little scope of improvement in the future. The other metal oxides like $\mathrm{MoO}_{3}$, iron oxide and $\mathrm{TiO}_{2}$ have been extensively studied and shown to be promising anode materials but require further evaluation in terms of material architectures and high rate capability.

Si nanowire arrays exhibit high initial capacities and capacities close to 1000 $\mathrm{mAhg}^{-1}$ at $0.2 \mathrm{C}$ rate for up to 100 cycles but only one third of the theoretical capacity of $\mathrm{Si}$ which shows that new material architectures have to be developed for further improvement. Ge, another material with higher mobility than Si has shown great promise but require further developments. In addition, intermetallic candidates are also attractive candidates. 3D nanoarchitectures of different materials exhibited high capacity retentions and good rate capability with cycling indicating that this is the preferred route to broaden the scope of LIBs and their performance. 


\section{CHAPTER 3}

\section{EXPERIMENTAL TECHNIQUES}

\subsection{Introduction}

This chapter discusses the techniques used for synthesizing the materials used in this study, tools used for identification and measurement of their physical properties, and electrochemical techniques used for determining the behavior of these materials as anodes in lithium ion battery (LIB).

A variety of chemical vapor deposition (CVD) techniques are used for synthesizing the materials. The metal oxide nanowires including $\mathrm{SnO}_{2}, \mathrm{MoO}_{3}$ and $\mathrm{WO}_{3}$ are synthesized using a hot wire chemical vapor deposition (HWCVD). Carbon microtubes (CMTs) are synthesized using metal organic chemical vapor deposition (MOCVD). The hybrid architectures are synthesized using plasma assisted microwave chemical vapor deposition (MWCVD). The as-synthesized as well as the post-lithiated material samples are characterized for their structure and morphology using scanning electron microscopy (SEM) and transmission electron microscopy (TEM). The crystal structure of the samples is studied using X-ray diffraction (XRD) and Raman spectroscopy (in-Via Renishaw micro-Raman system with a cooled CCD detector). The

surface area of the materials is determined using Brunauer-Emmett-Teller (BET) technique. The electrochemical behavior is determined using chronocoulometry, cyclic 
voltammetry and differential capacity measurements. The electrode fabrication and electrochemical cell are described in the later sections.

\subsection{Material synthesis}

\subsection{1 $\mathrm{SnO}_{2}$ nanowires}

$\mathrm{SnO}_{2}$ nanowires are synthesized on substrates in a CVD reactor. Gas phase synthesis of $\mathrm{SnO}_{2}$ nanowires is performed in a downstream microwave plasma reactor described below.

\subsubsection{1 $\mathrm{SnO}_{2}$ nanowires on substrates}

$\mathrm{SnO}_{2}$ nanowires are synthesized using reactive vapor deposition. These experiments are carried out in a low pressure CVD reactor with Sn metal (Aldrich) as the source. The source material is placed in an alumina coated tungsten (W) boat heater. The heater is covered with the substrate which in this case is quartz with minute openings for oxygen. The schematic of the reactor is shown in Figure 3.1.

The Sn metal is heated to a temperature of $1100^{\circ} \mathrm{C}$ with the quartz substrate measured at $900^{\circ} \mathrm{C}$ using a dual wavelength pyrometer. $\mathrm{SnO}_{2}$ nanowire synthesis is carried out in lean oxygen conditions using $5 \mathrm{sccm} \mathrm{O}_{2}$ and $200 \mathrm{sccm} \mathrm{H}_{2}$ at a pressure of 700 mtorr. The deposition is carried out for a duration of 30 minutes. The synthesized material is characterized by SEM, TEM, XRD and Raman spectroscopy to determine its physical structure, crystallinity and phase. 


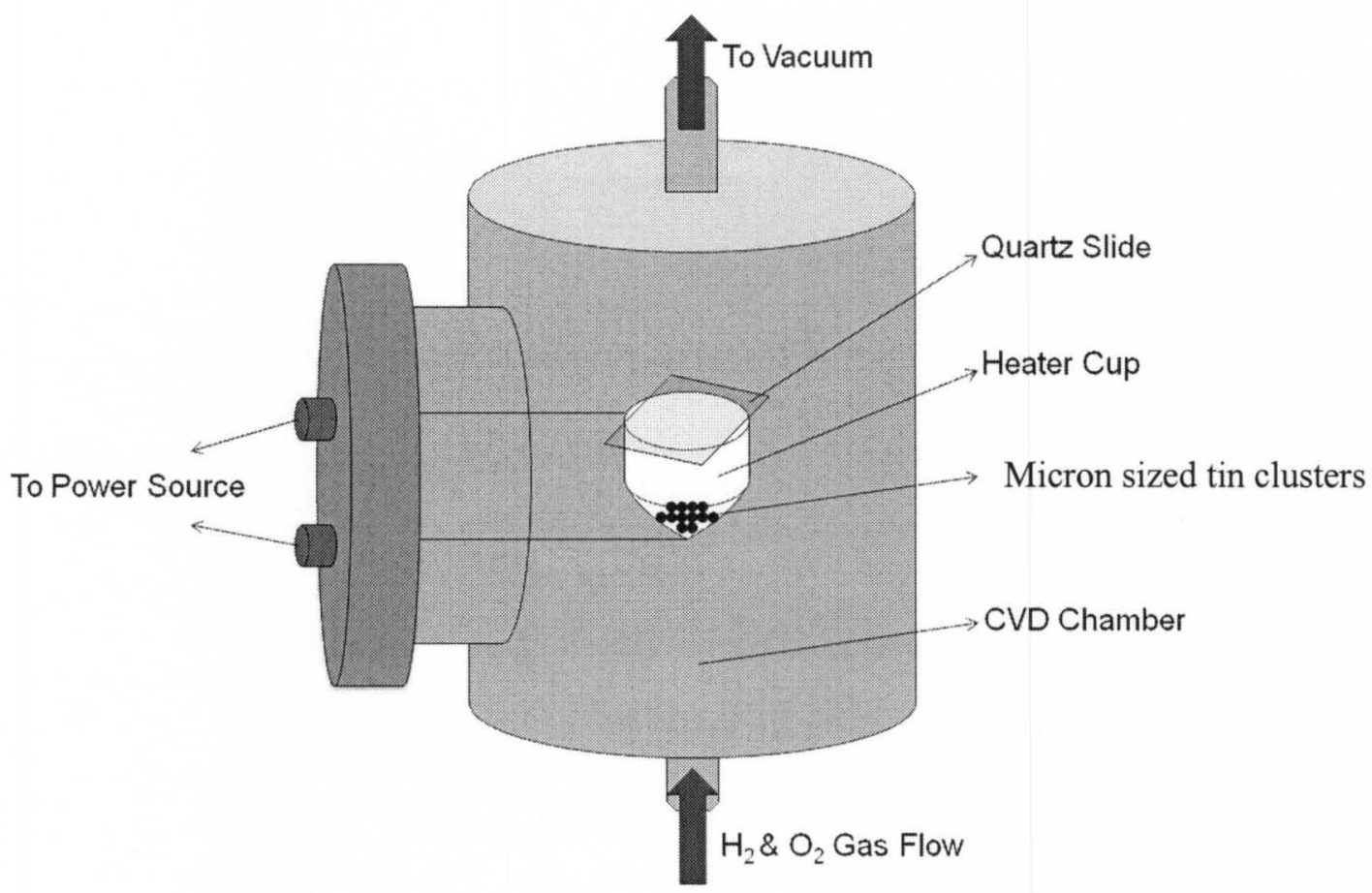

Figure 3.1: Schematic of the CVD reactor used for $\mathrm{SnO}_{2}$ nanowire synthesis.

\subsubsection{Gas phase production of $\mathrm{SnO}_{2}$ nanowire powders}

The schematic of the reactor used to produce nanowire powders is shown in Figure 3.2. The reactor is based on a microwave plasma jet discharge generated using a power source operating at $1.5 \mathrm{~kW}$ and $2.45 \mathrm{GHz}$ inside a vertical quartz tube. The reactor can be operated over a range of pressures ranging from a few torr to atmospheric pressure and at powers ranging from $300 \mathrm{~W}$ to $3 \mathrm{~kW}$. $\mathrm{SnO}_{2}$ nanowire synthesis is carried out at 2 $\mathrm{kW}$ using micron sized Sn metal powders at 10 slpm of air, $100 \mathrm{sccm}$ of $\mathrm{H}_{2}$ and $500 \mathrm{sccm}$ of $\mathrm{O}_{2}$ at atmospheric pressures. The gases enter the quartz tube from the top of the reactor and the metal powders are poured into plasma cavity zones which drop down by gravity. The plasma flame processes $\sim 3 \mathrm{~g}$ per minute of metal powder which translates to an equivalent production capacity of $4 \mathrm{~kg}$ of metal oxide NWs per day on continuous 
operation. The reactor can be operated continuously with a recycle stream for unreacted metal particles. The finer nanowire powders entrained by the gases are collected in the filter bags enhancing the quality of the synthesized nanowires. Experiments performed at higher plasma powers yield spherical, unagglomerated metal oxide nanoparticles.

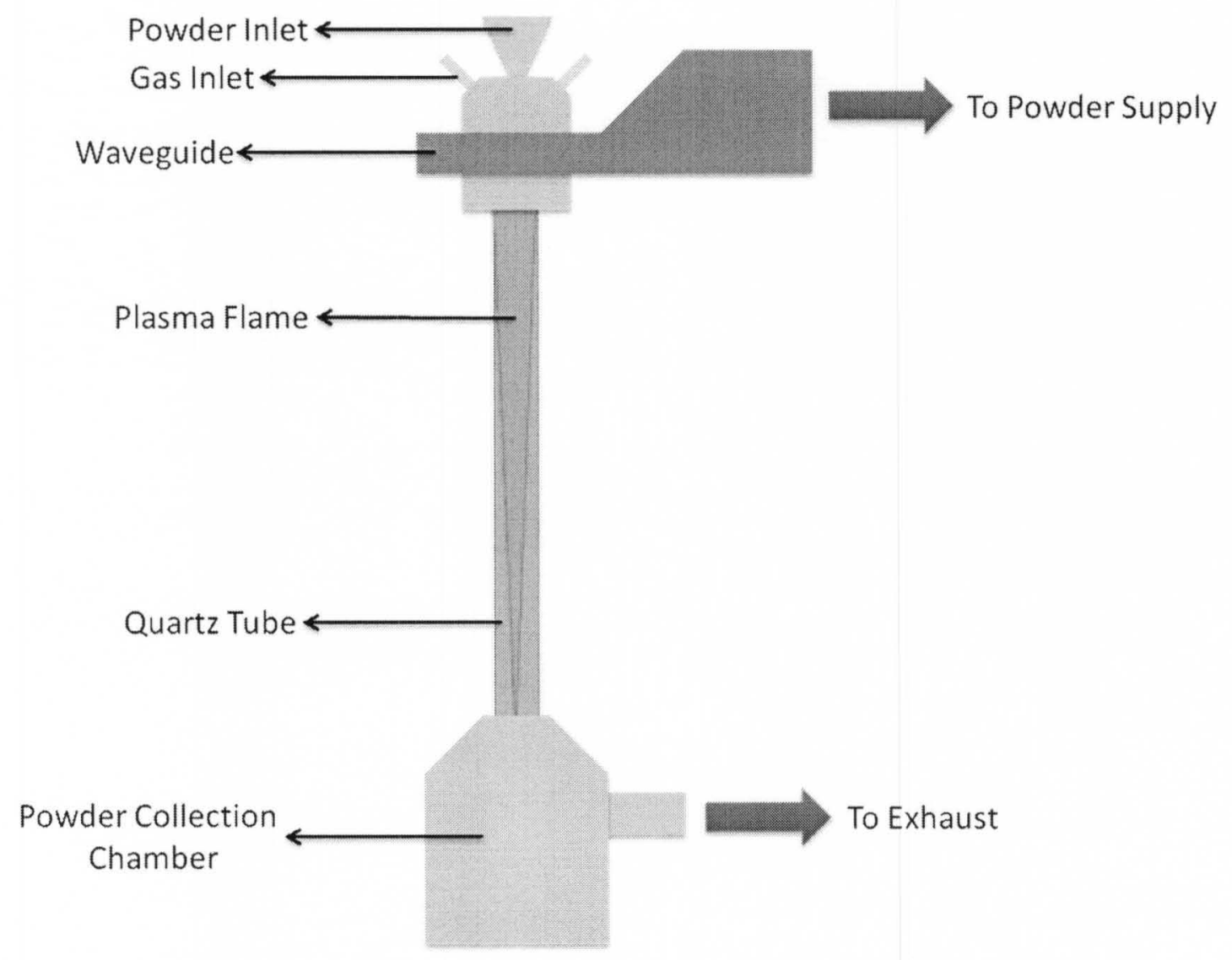

Figure 3.2: Schematic of downstream plasma reactor used for large scale synthesis of $\mathrm{SnO}_{2}$ nanowires.

The salient features of the reactor include:

- The production rate is $4 \mathrm{~kg} /$ day with $80-90 \%$ yield of NWs with a metal handling capacity of 3 grams/minute. 
- Capability of making different metal oxide nanowire powders inexpensively using micron scale metal particles.

- Bulk, fast, direct gas phase (no substrate, catalyst, template needed), contaminationfree, synthesis at atmospheric conditions (without expensive vacuum components) with recycling capacbility.

\subsection{2 $\mathrm{MoO}_{3}$ and $\mathrm{WO}_{3}$ nanowire arrays}

$\mathrm{MoO}_{3}$ and $\mathrm{WO}_{3}$ nanowire arrays are grown directly onto conducting substrates which gives rise to good conduction using a hot filament CVD reactor. The reactor utilizes metallic hot-filaments as a metal source and oxygen flow over the filaments to produce metal oxide vapors. The subsequent deposition of metal oxide vapors onto substrates kept at a distance and temperature leads to either thin films containing spherical crystals or vertical arrays of nanowires. Low amounts of oxygen partial pressure leads to the deposition of vertical arrays of metal oxide nanowires. In addition to $\mathrm{MoO}_{3}$ and $\mathrm{WO}_{3}$, the reactor has been shown to produce nanowire arrays with a number of materials systems such as nickel oxide, tantalum pentoxide, etc.

Figure 3.4 shows the schematic of HFCVD system. The HFCVD reactor consists of a 2 inch diameter quartz tube which is housed in a tube furnace. A $0.5 \mathrm{~mm}$ diameter coiled filaments of $\mathrm{W}$ and Mo are used as the sources for the respective metal oxide deposition. The metal oxide NW arrays have been reduced to yield metal NW arrays easily. The filaments were heated to temperatures ranging from 1773 to $2273 \mathrm{~K}$ measured using a dual wavelength pyrometer. The schematic of the HFCVD reactor is shown in Figure 3.3. The schematic shows the use of additional quartz boats which hold the 
substrate used for the deposition process (quartz in the case of $\mathrm{WO}_{3}$ nanowires and stainless steel in the case of $\mathrm{MoO}_{3}$ nanowires) and to prevent the deposition on the walls of the tube.

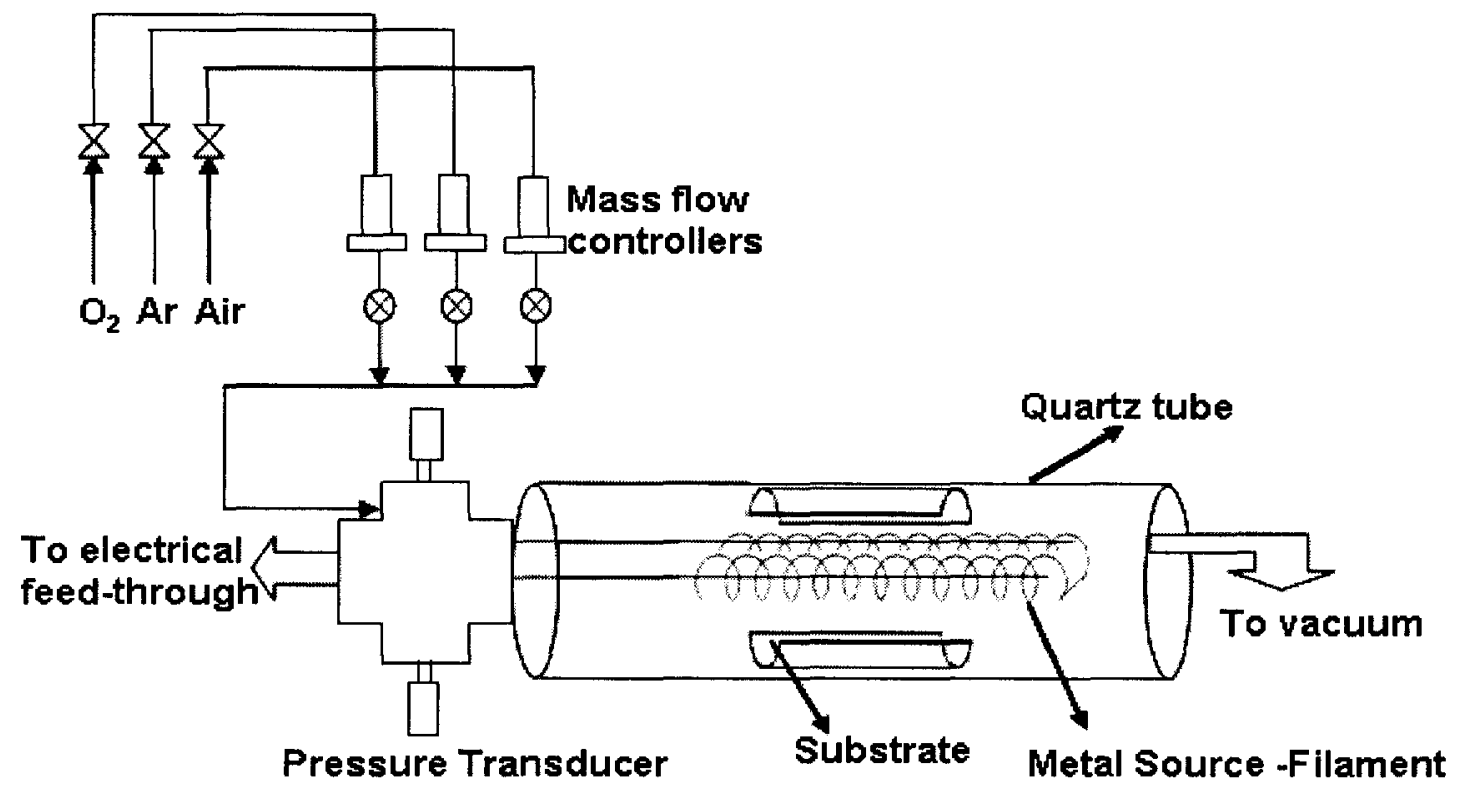

Figure 3.3: Schematic of the HFCVD system for the metal oxide nanowire array synthesis.

The feed gases which are either pure oxygen or a mixture of argon and air were mixed in appropriate ratios to obtain oxygen partial pressures ranging from 0.1 Torr to 10 Torr used for nanowire array synthesis. The nanowire density, morphology and the diameter can be controlled by the substrate temperature (controlled in turn by the filament temperature) and the oxygen flow into the system. The nanowire arrays are characterized using SEM, TEM, XRD and Raman spectroscopy. 


\subsubsection{Carbon microtubes}

CMTs are synthesized in a metal organic CVD reactor. The reactor consists of two separate components namely the precursor delivery system and the reaction chamber. The precursor delivery system is designed for independent flux control for up to four precursors simultaneously. Each precursor handling bay has a temperature control bath, controlled carrier gas supply, means to monitor and control the pressure in the precursor bubbler. All the lines feed into a single gas mixing port which leads into the reaction chamber. The reaction chamber is a water cooled stainless steel vacuum chamber equipped with pressure and temperature measurement accessories. All the gases enter the reactor through a shower head that allows for a uniform distribution of the precursors over the entire substrate area. Substrates are placed on a graphite holder that is heated through an induction heater. The schematic of the reactor is shown in Figure 3.5.

CMTs are synthesized on 6 in. silicon wafers at a substrate temperature of $700^{\circ} \mathrm{C}$. The chamber pressure was set to 50 Torr with a carrier gas (nitrogen) flow rate of 10 $\mathrm{sccm}$, and the precursor, trimethyl gallium (TMGa), was set at $0^{\circ} \mathrm{C}$. A 10 min experiment covered the entirety of the silicon wafer with CMTs, and the mass of the sample obtained for each run was more than $200 \mathrm{mg}$. CMTs were then scraped off the silicon wafers and dispersed in aqua regia to dissolve the gallium. Aqua regia was freshly prepared by making a solution of concentrated nitric acid (Fisher Scientific) and concentrated hydrochloric acid (Fisher Scientific) in a volumetric ratio of 1:3. The CMTs were allowed to stand in the acid solution for an hour before removing the settled deposit of the CMTs from the solution. The CMTs were then washed with water and then dried at $200^{\circ} \mathrm{C}$ for a couple of hours to obtain clean gallium free CMTs. 


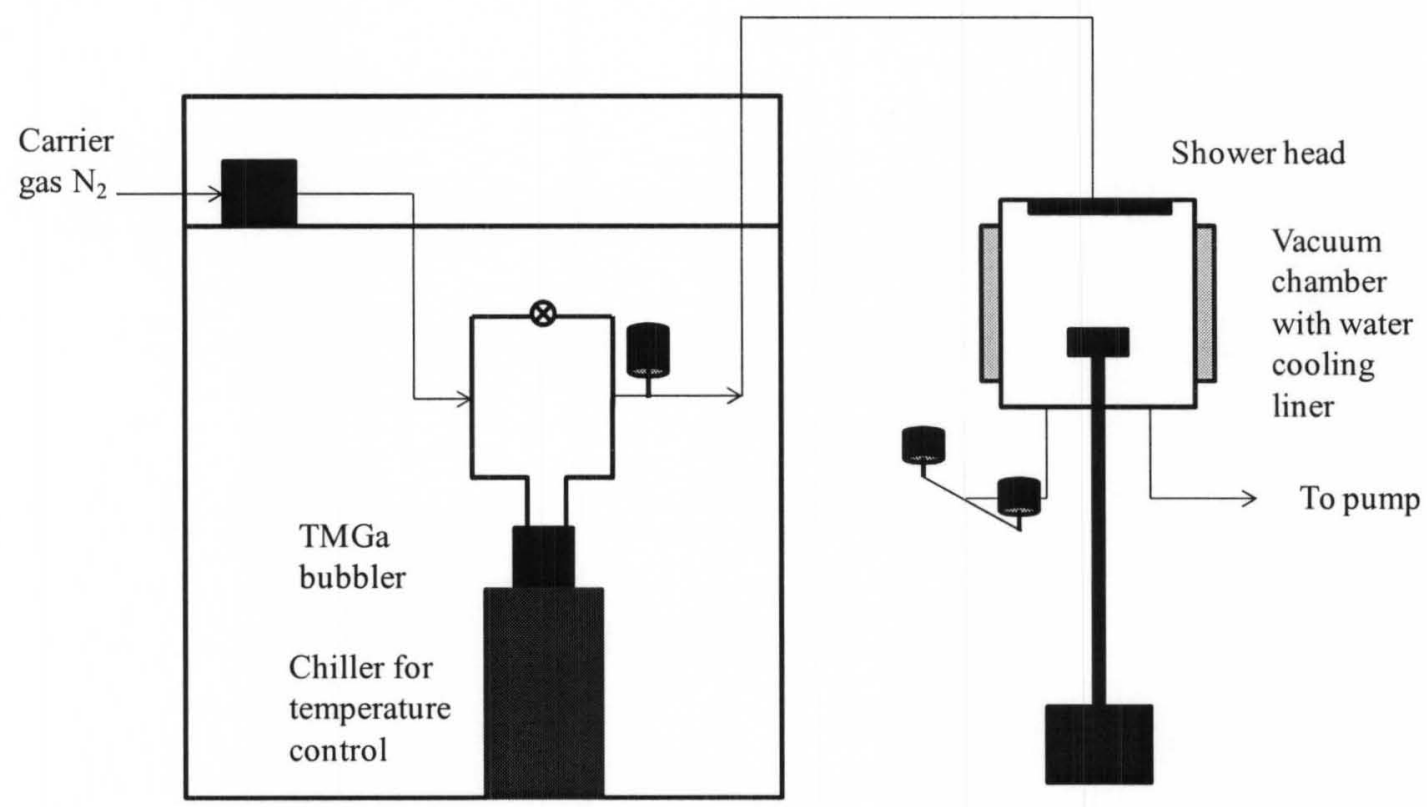

Figure 3.4: Schematic of MOCVD reactor indicating precursor delivery system along with the reaction chamber

\subsubsection{Hybrid architectured nanowires}

The as synthesized pure $\mathrm{SnO}_{2}$ nanowires are exposed to the $\mathrm{H}_{2}$ plasma in a microwave CVD reactor. The reaction is carried out at a power of $400 \mathrm{~W}$ for a duration of 10 minutes to obtain $\mathrm{Sn}$-nanocluster-covered $\mathrm{SnO}_{2}$ nanowires (hybrid architectured nanowires).

\subsection{Material characterization}

Nanowire morphology, diameter and density are determined using the FEI Nova 600 SEM. The crystal structure and nanowire orientation is studied using FEI Tecnai F20 with a Gatan 2002 GIF system TEM and Bruker D8 Discover, $\mathrm{Cu} \mathrm{K} \alpha$ radiation XRD. The material phase is determined using Raman spectroscopy with a HeNe laser (632.8 $\mathrm{nm})$ as an excitation source. Micromeretics TrisStar 3000 is used to determine the surface 
area of the nanomaterials using the nitrogen adsorption/desorption data. Post-lithiated samples are also characterized using the above tools for their crystallinity and morphologies.

\subsection{Lithium ion battery electrodes}

\subsubsection{Electrode fabrication}

The as synthesized arrays grown on conducting substrates are used directly for the electrochemical testing purposes. The nanowire powders are used as electrodes by making a slurry and coating onto conducting substrates. $\mathrm{SnO}_{2}$ nanowire powders, CMTs and hybrid architectures slurries are prepared by the standard procedure used in battery industry. The materials are mixed with poly(vinylidene fluoride) and carbon black in a weight ratio of 80:10:10, respectively, using 1-methyl-2-pyrrolidone (NMP) as the solvent. Carbon black is to enhance the conductivity of the electrode. The slurry is then coated onto a copper foil and hard pressed before drying the electrode in a vacuum oven at a temperature of $\sim 110^{\circ} \mathrm{C}$ overnight. The counter and reference electrodes are both lithium metal foils. The electrolyte consists of $1 \mathrm{M}$ lithium hexaflourophosphate (LiPF6) dissolved in a 1:1 volumetric ratio of ethylene carbonate (EC) and diethyl carbonate (DEC).

\subsubsection{Experimental setup}

All the experiments are carried out in a flooded cell in a three electrode configuration with the nanomaterial as the working electrode, lithium metal foil counter and reference electrodes as shown in Figure 3.6. This setup was assembled in a 2 inch quartz tube using conducting (copper/platinum) wires as current collectors for the electrodes. All the experiments are carried out in a purified argon inert atmosphere glove 
box (Vacuum Atmospheres Company). The photograph of glove box is shown in Figure 3.7.

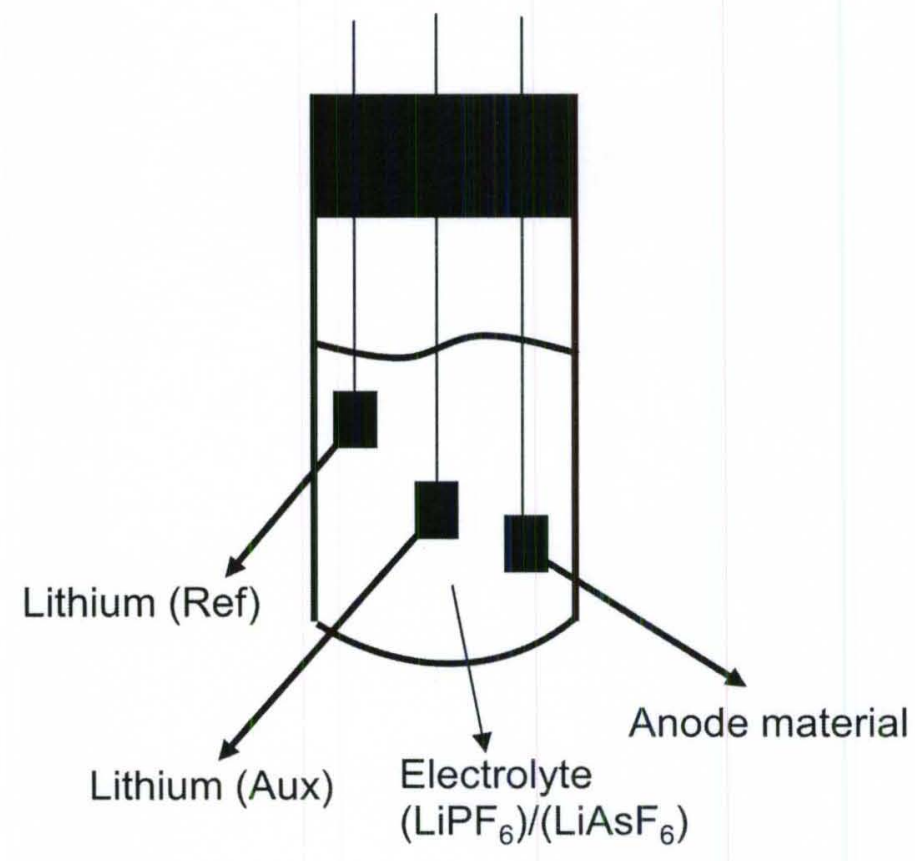

Figure 3.5: Schematic of the flooded three cell configuration.

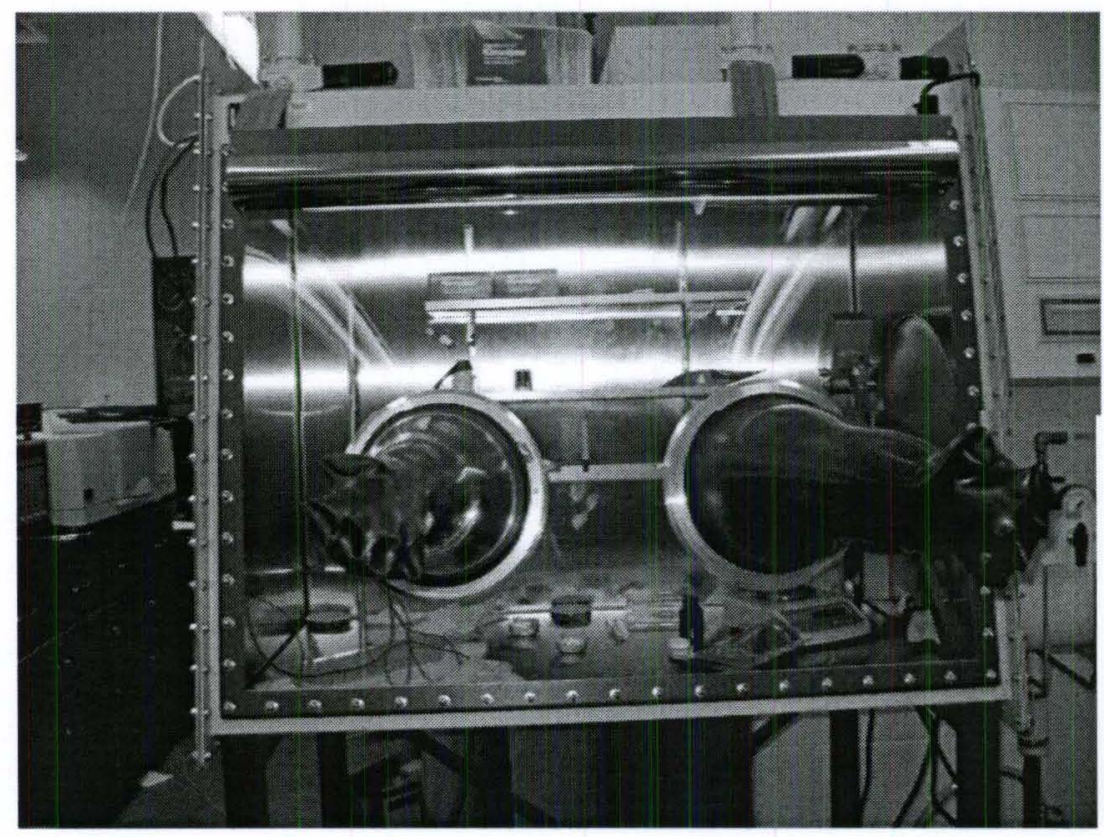

Figure 3.6: Photograph of the Vacuum Atmospheres Company glove box. 
The glove box is filled and maintained in ultra high purity argon atmosphere. The glove box is equipped with an purification system for the removal of oxygen and moisture. All the electrochemical studies are carried out at room temperature.

\subsubsection{Electrochemical testing}

All the electrochemical measurements are performed using an eDAQ e-corder and potentiostat.

\subsubsection{Constant current galvanostatic measurements}

This electrochemical technique involves passage of constant current between two electrodes in which the potential of one electrode is recorded versus the reference electrode. The potential decay with time is then used to calculate the specific capacity of the material using the equation:

\section{Specific Capacity $\left(\right.$ mAhg $\left.^{-1}\right)=($ current density $*$ time for potential decay $) /($ mass of material)

The polarity is reversed to measure the delithiation capacity. The capacity loss between the first discharge and charge cycle is an indication of the irreversible capacity, which is primarily due to the formation of the solid electrolyte interface (formed on the working electrode surface on interaction with the electrolyte which happens mostly during the first cycle (SEI)). This process of potential decay is continued over many cycles to obtain the stability of the material. The specific capacity of each lithiation and delithiation (charge and discharge) cycle is calculated to determine the capacity loss of the material with cycling. These studies are repeated at various current densities which 
provide an insight into the $\mathrm{Li}$ ion diffusion rates into and out of the material, which is of utmost importance when considering $\mathrm{Li}$ ion batteries for a variety of portable, transportation and grid stabilization applications. Figure 3.7 indicates a constant current curve between two potentials that takes place over a period of time and is used to calculate the specific capacity.

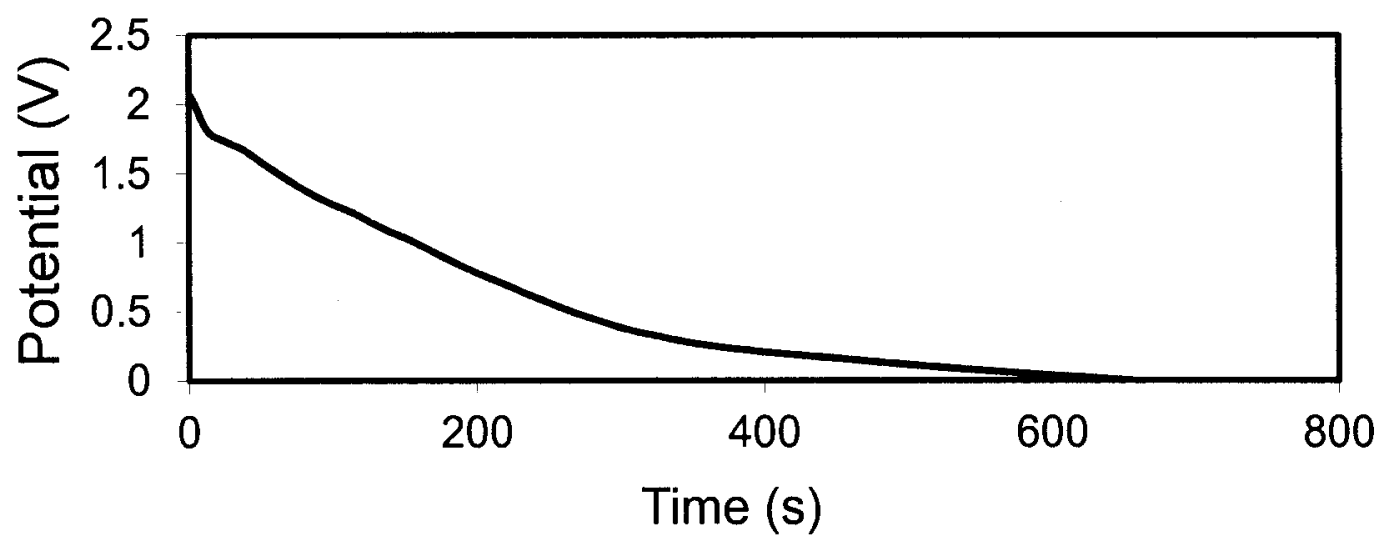

Figure 3.7: Typical curve used in determining the specific capacity.

\subsubsection{Differential capacity curves}

The specific capacity data is also used to determine the differential capacity. It is calculated using the equation below:

$$
d q / m d V=(I / m)([t(n+1)-t(n)] /[V(n+1)-V(n)])
$$

where "I" is the current, " $m$ " is the mass of the material, $V(n+1)$ and $V(n)$ are the adjacent voltage data points to the adjacent time data $t(n+1)$ and $t(n)$. The calculated data is plotted against the voltage, $\mathrm{V}$ and the peaks at different voltages are used to identify the different reactions occurring during the lithiation and de-lithiation processes. The unit of differential capacity is $\mathrm{mAhg}^{-1} \mathrm{~V}^{-1}$. The plots for different cycles are plotted against one 
another and the peaks in the plot are indicative of the coexistence of the different Li-alloy phases. Co-existence of different phases induces significant volume changes inside the material and destabilizes the electrode with cycling.

\subsubsection{Cyclic voltammetry}

Cyclic voltammetry (CV) is an important electrochemical technique for characterizing the electrode materials. The technique involves the application of linearly changing voltage to the working electrode ${ }^{133}$. The voltage scan window is chosen so that most of the electrode reactions would be encompassed. In order to better describe the CV principles, let us consider the following reversible reaction:

$$
O+n e^{-} \rightleftharpoons R
$$

The initial potential scan is best formulated as:

$$
E=E_{o}-v t
$$

where $E_{o}$ is the initial potential, $v$ is the scan rate expressed in $\mathrm{Vs}^{-1}$ and $t$ is the time. Similarly, the potential scan during the reverse sweep is represented as:

$$
E=E_{o}+v^{o} t
$$

where $v^{o}$ is the reverse scan rate and is essentially the same as scan rate $v$. The Nernst equation is as follows:

$$
E=E_{o}-\left(\frac{R T}{n F}\right) \ln \left(\frac{C_{O}}{C_{R}}\right)
$$

where $C_{O}$ and $C_{R}$ are the concentrations of the oxidized half and the reduced half respectively. Fick's law states that the material diffuses from a higher concentration to a lower concentration and obeys the law of diffusion as follows:

$$
F l u x=-D\left(\frac{\delta c}{\delta x}\right) \mid x=0
$$


where $D$ is the species diffusion coefficient and $\delta C / \delta x$ is the partial concentration derivative at the electrode surface. Fick's second law of diffusion expresses the concentration change rate with time as follows:

$$
\frac{\delta C}{\delta t}=D\left(\frac{\delta^{2} c}{\delta^{2} x}\right)
$$

According to Faraday's law, the charge is related to the moles undergoing reaction can be expressed as:

$$
Q=n N F
$$

where $Q$ is the charge, $F$ is Faraday constant, $N$ is the number of moles and $n$ is the number electron involved in the reaction. The current can thus, be calculated using the expression below:

$$
i=\frac{d Q}{d t}=-n A F(f l u x)=n A F D\left(\frac{\delta C}{\delta x}\right) \mid x=0
$$

This is the general current determination equation for most of the electrochemical experiments. Using the equations $3.4,3.5,3.6,3.7$ and 3.8 and combining them, a differential equation expression for the species flux to the surface of the electrode can be deduced and solved analytically to obtain a solution. CV usually uses a constant sweep rate for the entire scan which allows for the simple conversion time scale to a potential scale and then used to record current as a function of applied voltage. The peak current is then calculated using the expression ${ }^{133}$ :

$$
i_{P}=\left(2.69 X 10^{5}\right) n^{1.5} v^{0.5} D^{0.5} A C
$$

where $i_{P}$ is expressed in amperes, $A$ is the area of the electrode $\left(\mathrm{cm}^{2}\right)$ and $C$ is the concentration of the bulk (molesm $\left.{ }^{-3}\right)$. All the other terms are explained in the above discussion. The ideal separation between the peak currents of the cathodic and the anodic scans is 


$$
\Delta E=\frac{2.3 R T}{n F}
$$

However, if we assume that the analysis is performed at room temperature, equation 3.12 simplifies to and is expressed in $\mathrm{mV}$

$$
\Delta E=\frac{59}{n}
$$

All the equations above in conjunction can help in the determination of multiple entities including concentration and diffusion coefficient of the material ${ }^{133}$. Figure 3.8 shows a cyclic voltammetry curve indicating different lithiation, de-lithiation and SEI formation peaks which can help in deducing the lithiation characteristics.

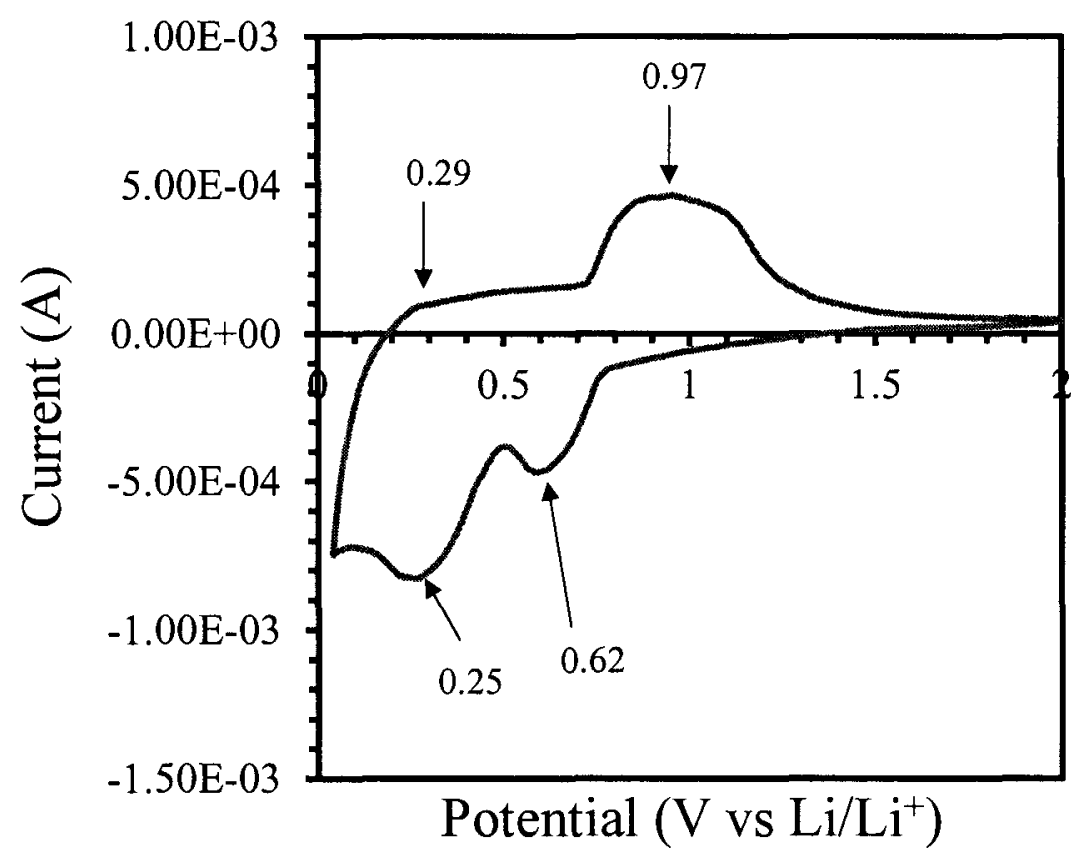

Figure 3.8: Cyclic voltammetry curve indicating the lithiation and de-lithiation peaks.

The following are the characteristics that can be well understood using the CV experiments and analysis: 
SEI layer formation: It is necessary to know the number of cycles during which this reaction takes place. It also indicates if this formation is reversible or irreversible.

Metal oxide reactions: Usually, when the metal oxide reacts with lithium, lithium intercalates into the metal oxide host and $\mathrm{Li}_{2} \mathrm{O}$ is formed during the first cycle followed by the subsequent alloying of $\mathrm{Li}$ with the metal host. It is necessary to study all the reactions to understand the material characteristics and if they are reversible.

Hybrid architectures chemistries: Especially in the case of hybrid architectures, it is essential to look at the reactions to understand the $\mathrm{Li}$ intercalation/alloying with the different material systems present.

CMTs/CNTs reactions: Although some of these have been well documented, we needed to study these to identify the mechanism of $\mathrm{Li}$ intercalation and de-intercalation into CMTs which have been studied for the first time.

\subsubsection{Post-lithiation analysis}

The post-lithiated samples are characterized using SEM, TEM, XRD and Raman spectroscopy for material physical structure, crystallinity and phase. 


\section{CHAPTER 4}

\section{HYBRID ARCHITECTURED NANOWIRES AS LITHIUM ION BATTERY ANODES}

\subsection{Introduction}

One dimensional nanomaterials and nanowires potentially offer shorter Lithium ion diffusion path lengths, electronic conducting pathways, better strain accommodation and high surface area per unit volume which makes them best suitable for both anodes and cathodes in Li-ion batteries. Nanowires therefore, are the architectures for the improvement of specific capacity, power, cycleability and life of Li-ion batteries.

Tin oxide is an interesting anode material owing to low lithiation potential, semiconducting properties along with high theoretical capacity $\left(781 \mathrm{mAhg}^{-1}\right)$. However, different $\mathrm{SnO}_{2}$ architectures including the thin films and nanoparticles have shown capacities of $\sim 400-500 \mathrm{mAhg}^{-1}$ but undergo degradation with cycling. Lithium alloying and de-alloying from $\mathrm{SnO}_{2}$ anode causes significant volume changes (259\%) leading to material segregation and crystallographic deformation. This breaks down conductivity channels inside the material resulting in capacity loss with cycling. Numerous attempts to improve the stability of the electrodes using thinner films and nanoparticles have only resulted in higher initial capacities with little improvement in the stability with cycling. Similarly, $\mathrm{SnO}_{2}$ nanorods, $\mathrm{SnO}_{2} / \mathrm{In}_{2} \mathrm{O}_{3}$ heterosructures, amorphous tin based oxide and 
$\mathrm{Sn} / \mathrm{SnO}_{2}$ composites report high initial capacities in the range of $700-550 \mathrm{mAhg}^{-1}$ but the capacities quickly fade to $300-600 \mathrm{mAhg}^{-1}$ in 50 cycles $^{74,75,78}$. Therefore, it is important to study and understand the performance of different $\mathrm{SnO}_{2}$ architectures. $\mathrm{SnO}_{2}$ nanowires, mixed metal-metal oxides $\left(\mathrm{Sn} / \mathrm{SnO}_{2}\right)$ and $\mathrm{Sn}$-nanocluster-covered $\mathrm{SnO}_{2}$ nanowires have all been investigated for their stability with cycling and capacity retention. This chapter discusses the reduction in the chemical and mechanical degradation of the composite materials composed of $\mathrm{Sn}$ and $\mathrm{SnO}_{2}$ with the addition of $\mathrm{Sn}$. The differences in their performance have been evaluated and explained using different electrochemical techniques like cyclic voltammetry, galvanostatic cycling and differential capacity measurements. Numerous techniques, such as SEM, TEM, Raman and XRD were used to compare the mechanical integrity of the materials before and after lithiation. A model has been proposed to understand the differences in performance.

\subsection{One-dimensional tin oxide nanowires as anodes in LIBs}

\subsubsection{Large scale synthesis}

$\mathrm{SnO}_{2}$ nanowires are synthesized using two different schemes. Traditionally, chemical vapor deposition (CVD) is the route for nanowire synthesis. The synthesis involves the use of tin metal as the source which is resistively heated at $1100{ }^{\circ} \mathrm{C}$ in lean oxygen conditions $\left(5 \mathrm{sccm} \mathrm{O}_{2}\right.$ and $\left.200 \mathrm{sccm} \mathrm{H}_{2}\right)$ at 700 mTorr. A detailed synthesis procedure has been presented in Chapter 3. The as synthesized nanowires have diameters that are ranging from $50-200 \mathrm{~nm}$ and are several microns long. The growth in this mode occurs by self catalytic vapor-liquid-solid (VLS) mechanism. On heating, Sn vaporizes and deposits as crystals on the quartz substrate. These Sn crystals act as catalysts and 
interact with oxygen vapors leading to the growth of $\mathrm{SnO}_{2}$ nanowires in one-dimension. The first image in Figure 4.1 shows the SEM image of micron long $\mathrm{SnO}_{2}$ nanowires grown on quartz, whereas the second image indicates that the nanowire diameters are less than $100 \mathrm{~nm}$.
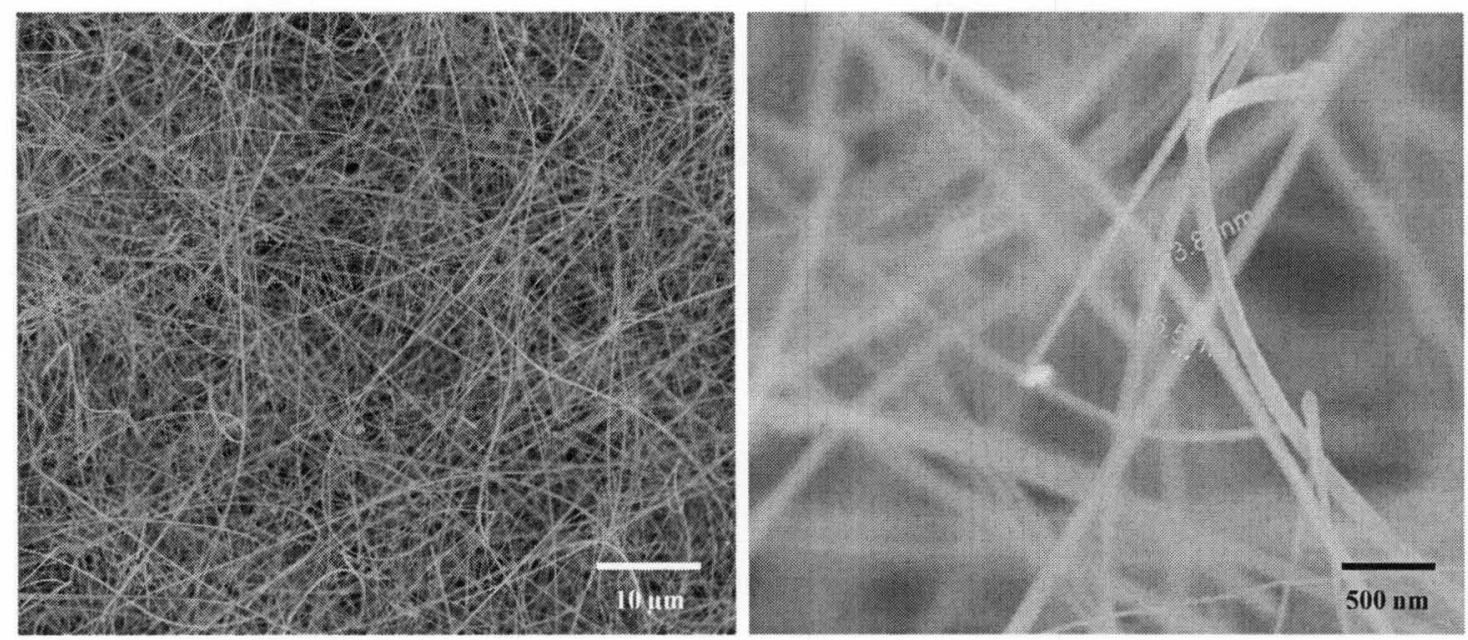

Figure 4.1: $\mathrm{SEM}$ images of the as synthesized $\mathrm{SnO}_{2}$ nanowires grown directly on quartz at $1100^{\circ} \mathrm{C}$.

Large scale synthesis of $\mathrm{SnO}_{2}$ nanowire powders is carried out in a downstream plasma reactor. This process follows the gas phase, direct oxidation of tin powder in microwave plasma. The resulting tin oxide nanowires are free of any foreign metal contamination that otherwise may hinder their electrochemical performance. The reactor is based on a microwave plasma jet discharge generated using a power source inside a vertical quartz tube. $\mathrm{SnO}_{2}$ nanowire synthesis in this reactor is carried out at $2 \mathrm{~kW}$ and at atmospheric pressures. The gases enter the quartz tube from the top of the reactor and the metal powders are poured into the plasma cavity zones which drop down by gravity. As the metal powder falls through the plasma flame region, it heats up, melts and reacts with 
the reactive species present in the plasma and solidifies quickly as it goes past the flame region. The nanowire powder is collected in a cup at the quartz tube bottom. $\mathrm{SnO}_{2}$ nanowires synthesized by either of the processes are quite similar and differ only in the fact that the downstream plasma reactor can synthesize gram quantities. The next section presents the physical and chemical analysis of the nanowires.

\subsubsection{Structural properties}

The X-ray diffraction spectrum of $\mathrm{SnO}_{2}$ nanowires shown in Figure 4.2 corresponds to the tetragonal rutile structure of bulk $\mathrm{SnO}_{2}$ (JCPDS 21-1250).

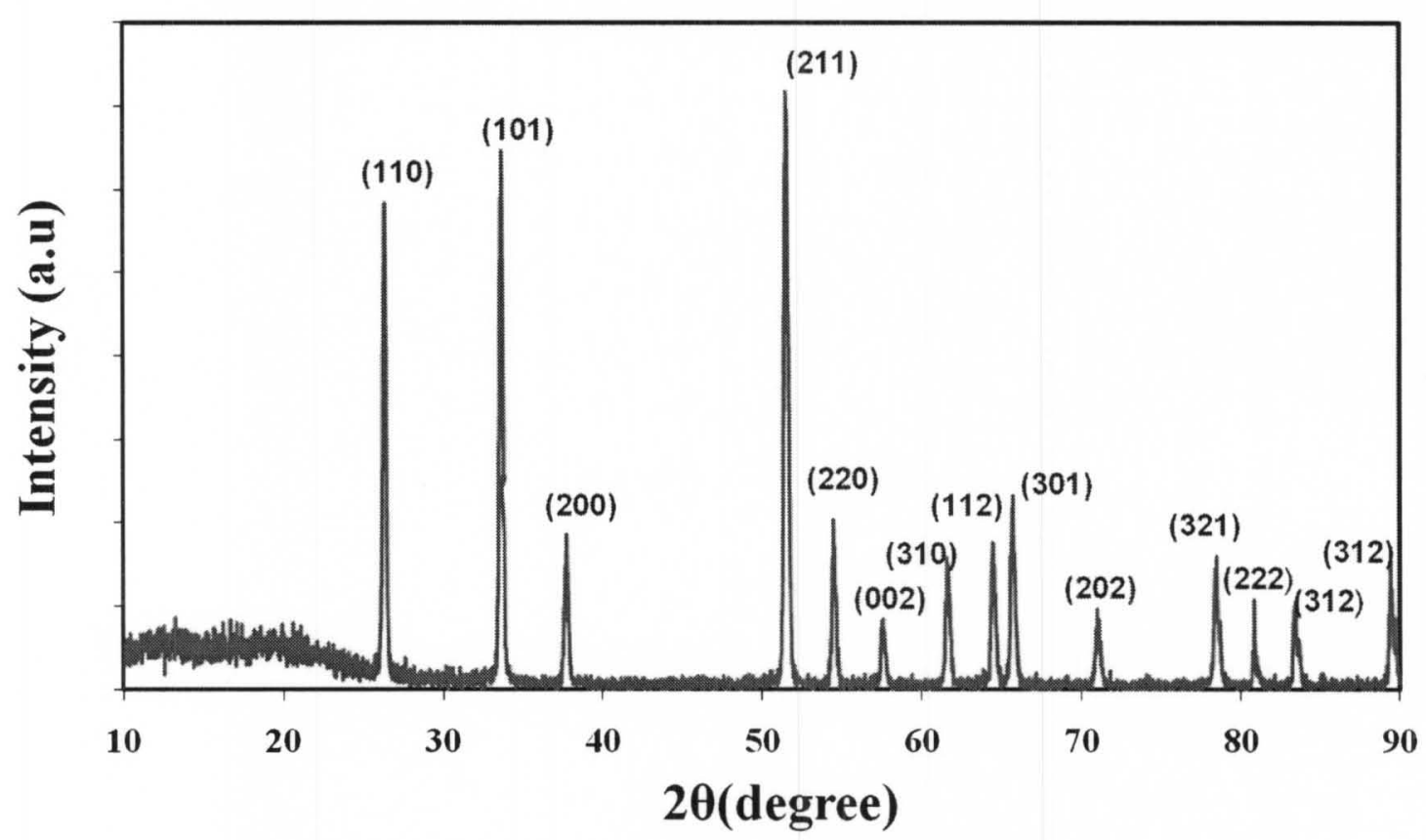

Figure 4.2: $\mathrm{XRD}$ spectra of pure phase $\mathrm{SnO}_{2}$ nanowires indicating their crystalline nature. 


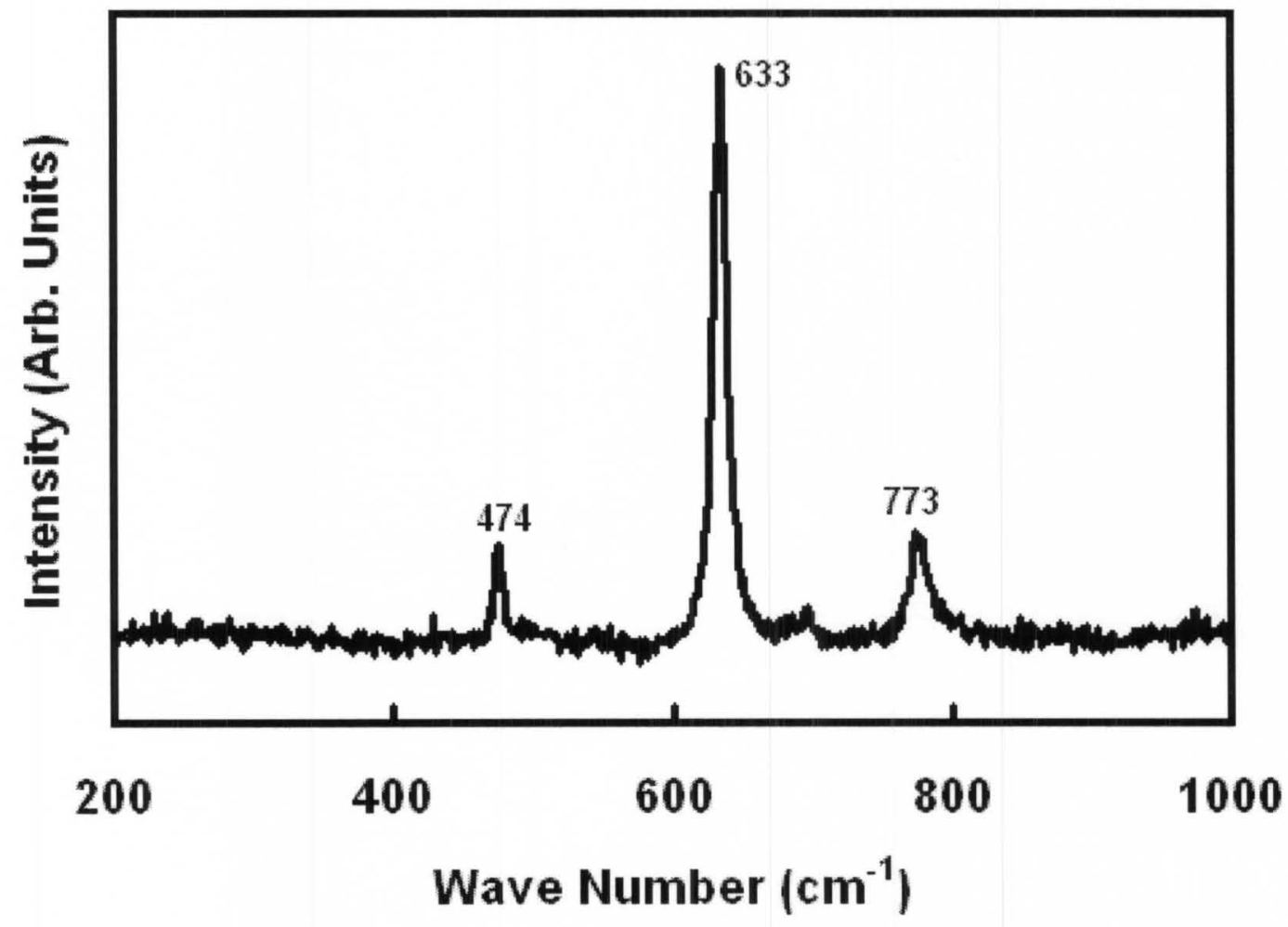

Figure 4.3: Raman spectrum of pure phase $\mathrm{SnO}_{2}$ nanowires.

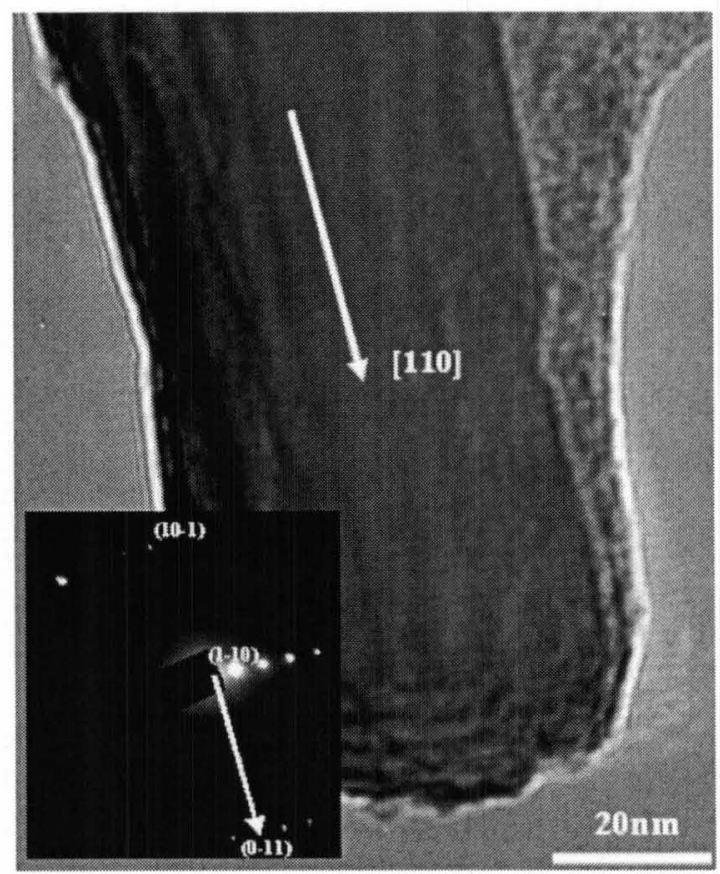

Figure 4.4: TEM image indicating the growth direction of a $\mathrm{SnO}_{2}$ nanowire. 
Raman spectrum of the as synthesized nanowires shows three peaks at 474,633 and $773 \mathrm{~cm}^{-1}$ corresponding to the $E_{g}, A_{1 g}$ and $B_{2 g}$ vibration modes, which match well with those of rutile phase bulk $\mathrm{SnO}_{2}$. The Raman spectrum is presented in Figure 4.3. The TEM image of a $\mathrm{SnO}_{2}$ nanowire and the corresponding diffraction pattern shown in Figure 4.4 indicate that the nanowire has a [110] growth direction.

\subsubsection{Electrochemical properties}

Galvanostatic studies are conducted on $\mathrm{SnO}_{2}$ nanowires over a potential window of 0.01 to $2.2 \mathrm{~V}$ (versus $\mathrm{Li} / \mathrm{Li}^{+}$). $\mathrm{SnO}_{2}$ reacts with lithium in a two step process. In the first step, $\mathrm{SnO}_{2}$ reacts with $\mathrm{Li}$ for the formation of metallic tin regions inside a $\mathrm{Li}_{2} \mathrm{O}$ matrix. The next step involves the lithium alloying with metallic tin and de-alloying which is a reversible process which is measured as the specific capacity of the battery. The reaction is as follows ${ }^{79}$ :

$$
\begin{gathered}
\mathrm{SnO}_{2}+4 \mathrm{Li}^{+}+4 e^{-} \rightarrow 2 \mathrm{Li}_{2} \mathrm{O}+\mathrm{Sn} \\
\mathrm{Sn}+x \mathrm{Li}^{+}+x e^{-} \leftrightarrow \mathrm{Li}_{x} \operatorname{Sn}(0 \leq x \leq 4.4)
\end{gathered}
$$

As mentioned earlier in Chapter 2, metallic tin undergoes enormous volume changes on lithium insertion and removal which leads to cracking and loss of electronic conductivity within the material. This results in continuous decrease in the capacity with cycling. The specific capacity testing is performed at a current density of $100 \mathrm{mAg}^{-1}$. As shown in Figure 4.5 this material shows a high initial capacity of $2470 \mathrm{mAhg}^{-1}$ but dropped down to $1032 \mathrm{mAhg}^{-1}$ in the next cycle indicating a large loss in capacity due to a) formation of amorphous $\mathrm{Li}_{2} \mathrm{O}$ matrix which is irreversible under the present conditions and b) formation of solid electrolyte interface (SEI) layer. The capacity quickly fades to 
lower than $200 \mathrm{mAhg}^{-1}$ in the first 10 cycles and is constant for the next 30 cycles. The initial high capacity is attributed to the high surface area to volume ratio in 1D nanowire which provides additional lithium alloying sites. The smaller diameter nanowires also offer short diffusion lengths for $\mathrm{Li}$ ions thus improving the electronic conductivity.

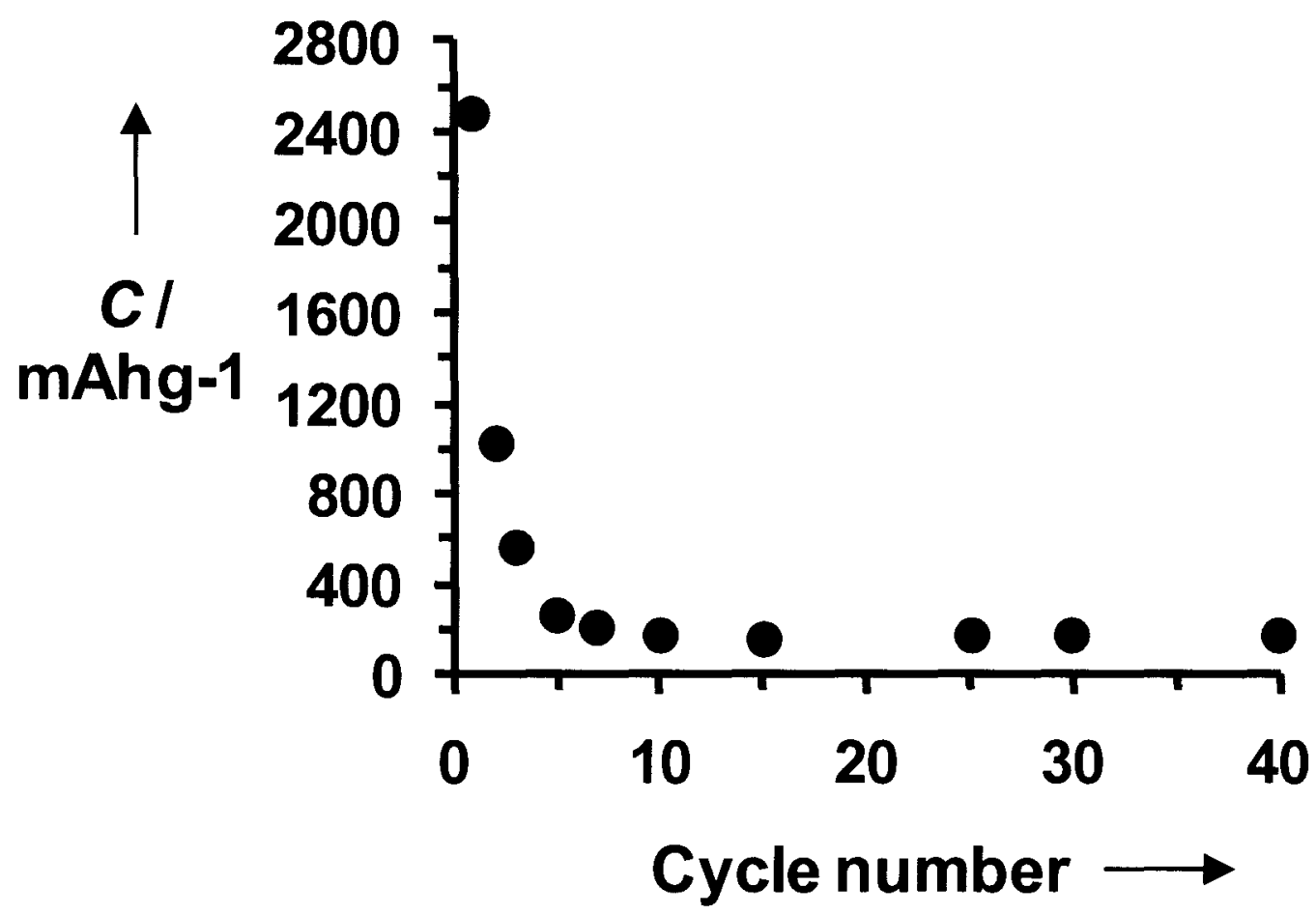

Figure 4.5: Specific capacity retention of pure $\mathrm{SnO}_{2}$ nanowires with cycling for up to 40 cycles.

In order to better understand the mechanism of $\mathrm{Li}$ ion storage in $\mathrm{SnO}_{2}$ nanowires, cyclic voltammetry (CV) is performed and presented in Figure 4.6. The first discharge cycle shows two apparent peaks at 1.11 and $0.8 \mathrm{~V}$ which correspond to the formation of $\mathrm{Li}_{2} \mathrm{O}$ (Equation 1 above) and the electrolyte decomposition ${ }^{134-136}$. These two peaks give 
rise to the large irreversible capacity loss in the first cycle. A peak at $0.84 \mathrm{~V}$ is seen from the $2^{\text {nd }}$ to the $4^{\text {th }}$ cycle which shows that the irreversible reaction takes place until $4^{\text {th }}$ cycle. The single crystalline structure of the $\mathrm{SnO}_{2}$ nanowires might slow the lithiation and de-lithiation thus enabling the formation of $\mathrm{Li}_{2} \mathrm{O}$ beyond first cycle. In addition, the volume expansion leads to the formation of new surface which serve as sites for electrolyte decomposition after the first cycle. The peak at $0.2 \mathrm{~V}$ during the discharge cycle corresponds to the formation of Li-Sn alloy as shown in Equation $4.2^{137}$. Specifically, the peak corresponds to the formation of $\mathrm{Li}_{4.4} \mathrm{Sn}$ phase which is the maximum limit of $\mathrm{Li}$ that can be alloyed with $\mathrm{Sn}^{137}$. The charge curve shows a single peak at $0.6 \mathrm{~V}$ corresponding to $\mathrm{Li}_{7} \mathrm{Sn}_{2}$ phase, which is $3.5 \mathrm{Li}$ extracted per $\mathrm{Sn}$ atom.

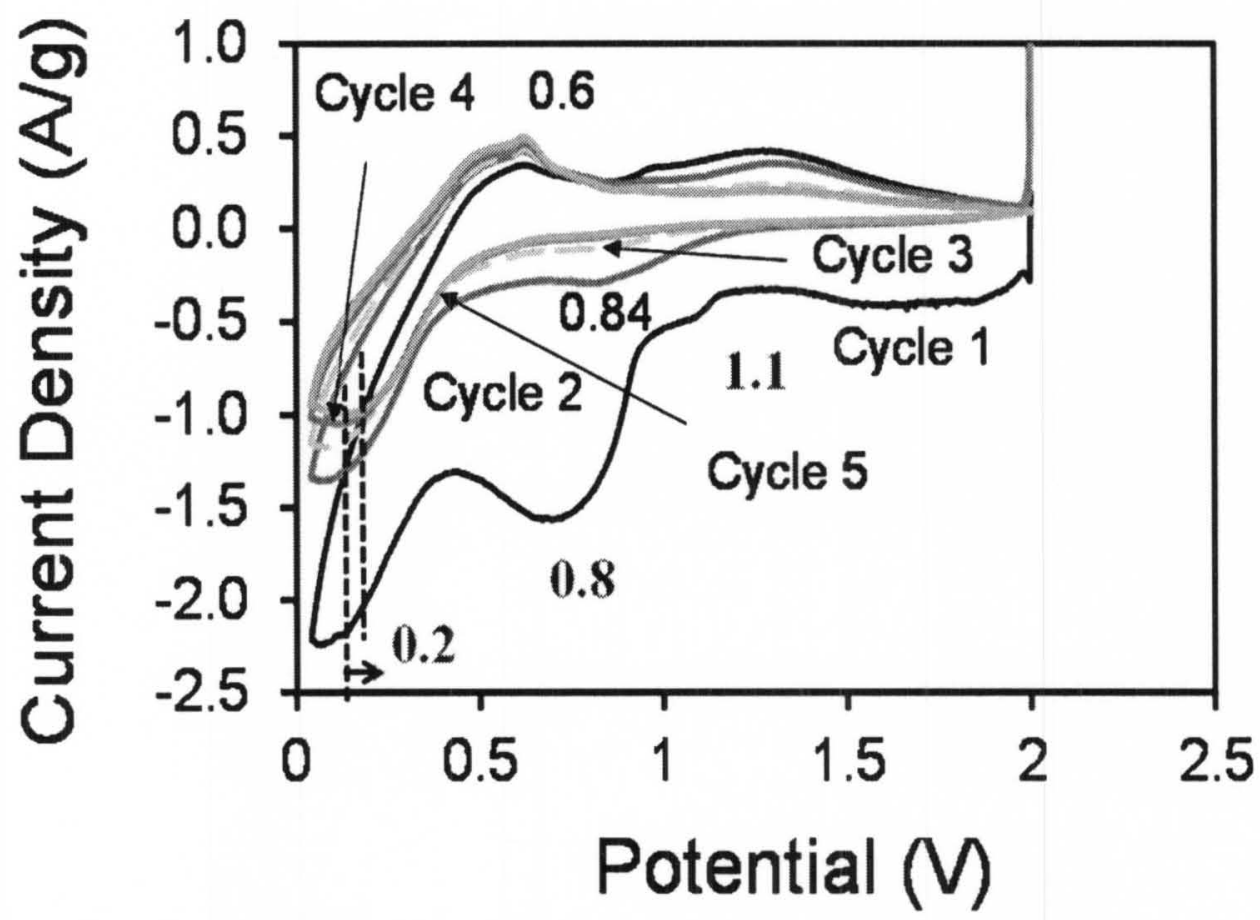

Figure 4.6: Cyclic voltammetry of $\mathrm{SnO}_{2}$ nanowires for the first 5 cycles with peak positions at a rate of $0.1 \mathrm{mVs}^{-1}$. 


\subsubsection{Post lithiation characteristics}

Figure 4.7 shows the comparison of XRD spectra of $\mathrm{SnO}_{2}$ nanowires after 40 charge-discharge cycles and the as-synthesized $\mathrm{SnO}_{2}$ nanowires. After lithiation, it can be seen that the majority of the $\mathrm{SnO}_{2}$ nanowires are converted to pure tin (220) phase as shown in the XRD spectrum along with some unreacted $\mathrm{SnO}_{2}$ phases.

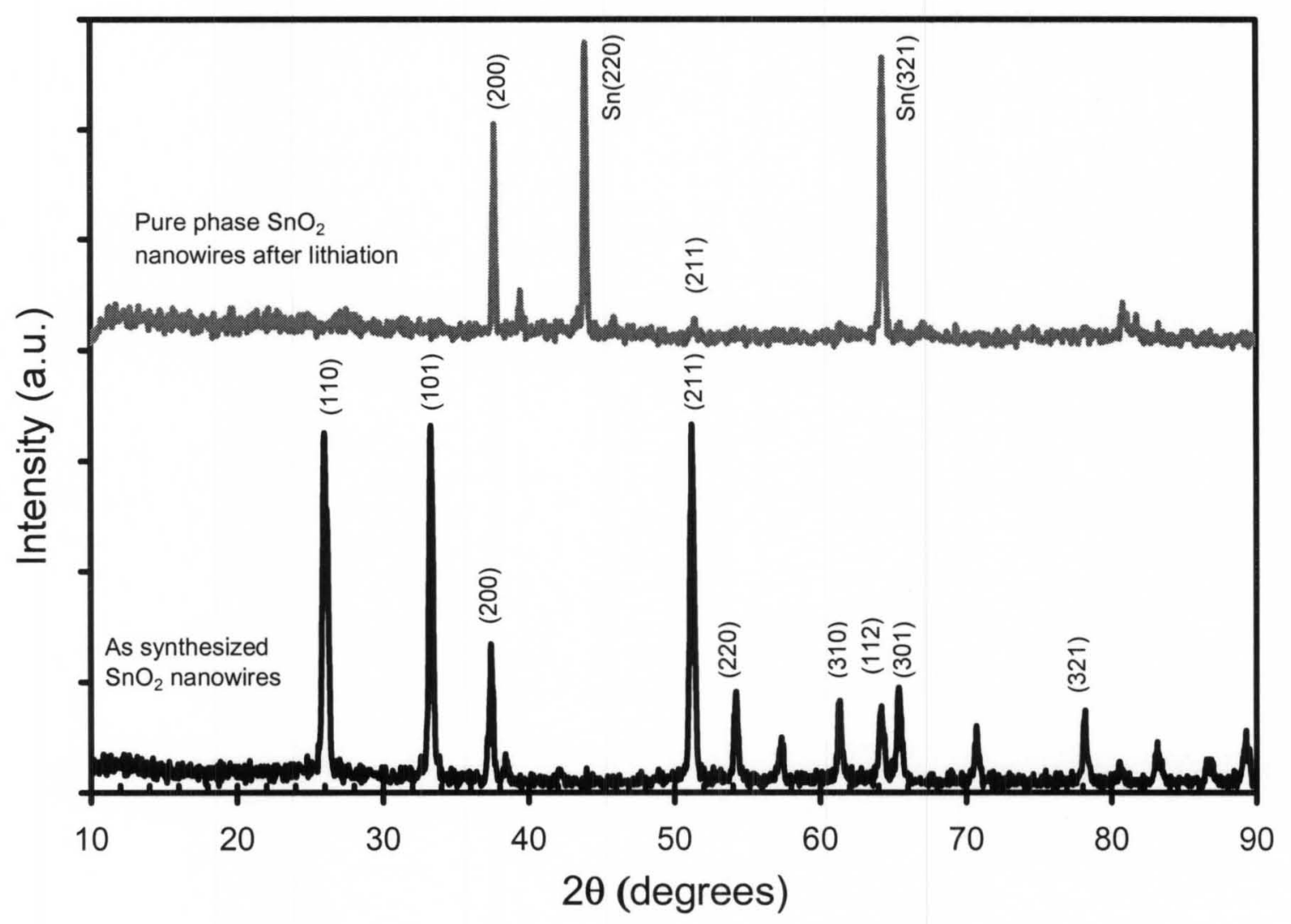

Figure 4.7: XRD comparison of pre and post-lithiated $\mathrm{SnO}_{2}$ nanowires.

The formation of Sn confirms that the nanowires follow the reaction mechanism presented earlier. Figure 4.8 shows the SEM images of the material after 40 cycles of lithiation. 


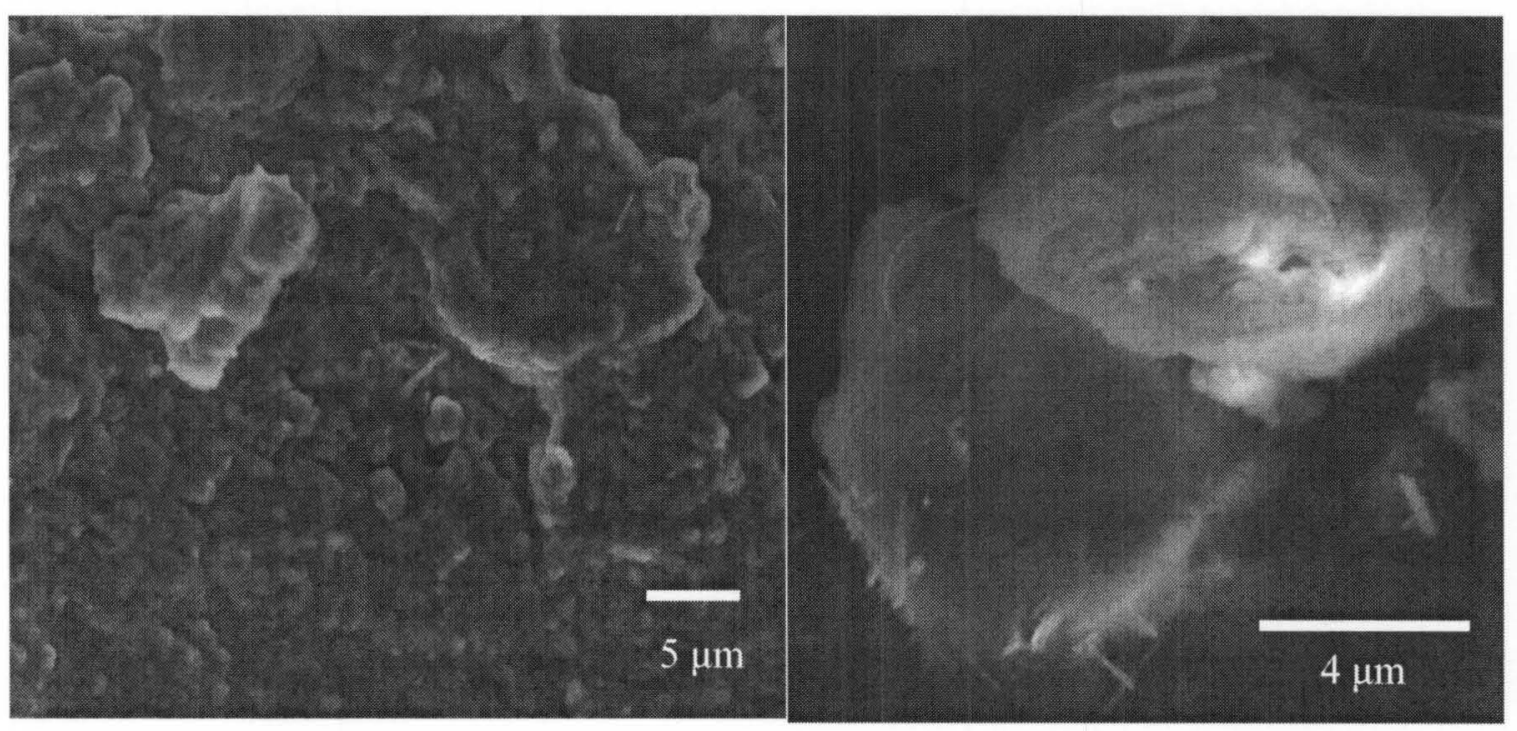

Figure 4.8: $\mathrm{SEM}$ images of $\mathrm{SnO}_{2}$ nanowires after lithiation showing the crumbled nanowires.

The reasons for an irreversible capacity loss during the first cycle are due to the reaction of $\mathrm{SnO}_{2}$ with $\mathrm{Li}$ ions, electrolyte decomposition and formation of solid electrolyte interface. Pure $\mathrm{SnO}_{2}$ materials undergo reduction to $\mathrm{Sn}$ during the first cycle of lithiation and de-lithiation. Repeated cycling on this anode leads to mechanical disintegration of the $\mathrm{Sn}$ particles leading to loss in electronic conductivity and $\mathrm{Sn}$ segregation with a loss of active surface area and in turn losing capacity. Particularly in the case $\mathrm{SnO}_{2}$ nanowires, $\mathrm{Sn}$ agglomerates destroy the nanowire architecture giving rise to big $\mathrm{Sn}$ globules. Other researchers have shown that the as-synthesized $\mathrm{SnO}_{2}$ nanowire samples exhibit capacity retention over a range of values $(166-300 \mathrm{mAh} / \mathrm{g})$, the higher capacity retention in some cases is attributed to the presence of excess Sn metal around the nanowires. $\mathrm{SnO}_{2}$ nanowires show great promise as high capacity anode materials but suffer severe mechanical degradation which makes them highly unstable with cycling. 
Hence, it is necessary to look at further modifications to the nanowires to improve the cycling stability along with increasing the capacity.

\subsection{Metal nanocluster covered metal oxide nanowires (hybrid architectures) as high} capacity anodes for LIBs

\subsubsection{Concept of hybrid architectures}

Here, we present a unique and simple generic design of hybrid structures involving metal nanoclusters covered metal oxide nanowires as stable anode materials with high reversible capacity. The design principle for the proposed hybrid structures is that metal oxide nanowires are covered with metal nanoclusters with enough spacing between the nanoclusters to accommodate the volume changes of metal nanoclusters during alloying and de-alloying thereby preventing the nanocluster agglomeration. The faster electron transport through the underlying metal oxide nanowires is expected to allow for efficient Li alloying and de-alloying while the exposed metal nanoclusters and nanowire surfaces serve as $\mathrm{Li}$ alloying sites. This design concept is illustrated in this study for $\mathrm{Sn}$ nanocluster covered $\mathrm{SnO}_{2}$ nanowires ${ }^{138}$.

\subsubsection{Synthesis and physical characteristics of $\mathrm{Sn}$ nanocluster covered $\mathrm{SnO}_{2}$ nanowires}

The design consists of decorating the $\mathrm{SnO}_{2}$ nanowires with $\mathrm{Sn}$ nanoclusters which are spaced apart 1.4 times the diameter of Sn nanoclusters with the spacing between them accommodating the $259 \%$ volume expansion of $\mathrm{Sn}$ when it forms $\mathrm{Li}-\mathrm{Sn}$ alloys. The conceptual design is presented in Figure 4.9. $\mathrm{SnO}_{2}$ nanowires are synthesized using the 
techniques discussed in Chapter 3. The as synthesized $\mathrm{SnO}_{2}$ nanowires are reduced in a microwave assisted plasma deposition reactor operating at $400 \mathrm{~W}$ in $70 \mathrm{sccm} \mathrm{H}_{2}$ at a pressure of 25 mtorr.

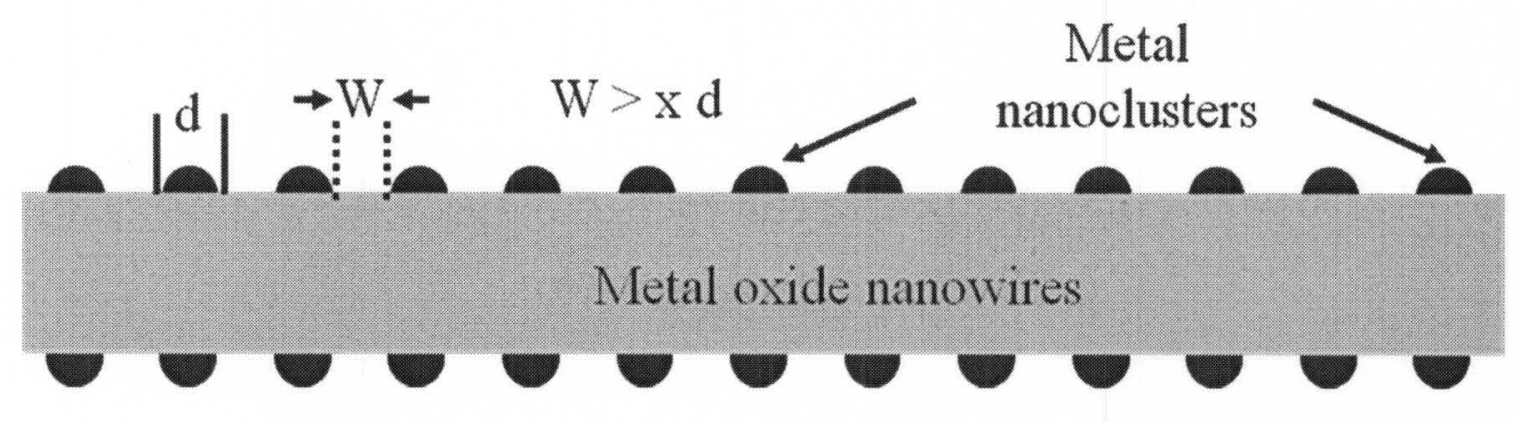

Figure 4.9: Schematic of metal-nanocluster-covered metal oxide nanowire with adequate spacing to accommodate volume expansion.

Figure 4.10 shows the SEM images of hybrid architectures which clearly indicate $\mathrm{SnO}_{2}$ nanowires covered with $\mathrm{Sn}$ nanoclusters.

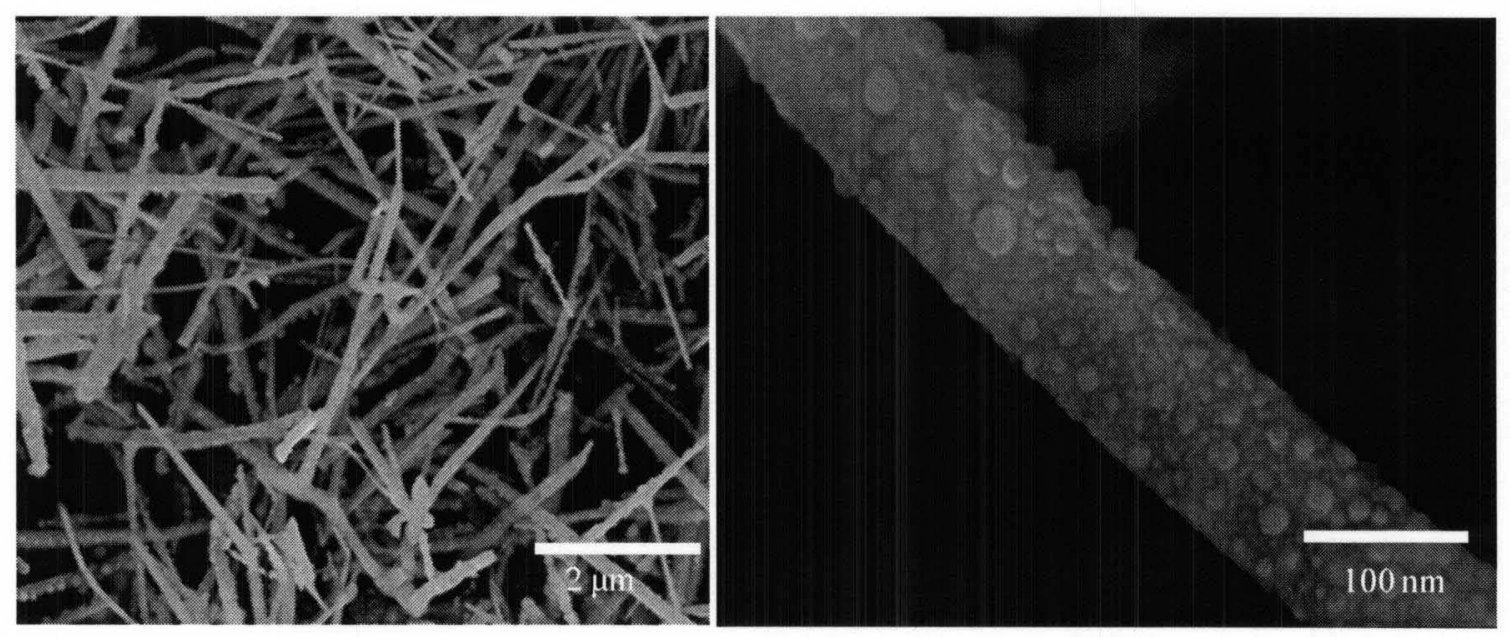

Figure 4.10: $\mathrm{SEM}$ images of $\mathrm{Sn}$-nanocluster-covered $\mathrm{SnO}_{2}$ nanowires with $\mathrm{Sn}$ nanoclusters clearly visible on the surface. 
It can be seen from the image that the average nanowire diameter decreases as a result of plasma exposure to $\sim 100 \mathrm{~nm}$. Sn nanoclusters are also clearly visible on the periphery of the nanowires with average diameters of $\sim 15 \mathrm{~nm}$. TEM and HRTEM images in Figure 4.11 show the hybrid architectures confirming the presence of $15 \mathrm{~nm}$ nanoclusters on the surface. In addition, the HRTEM image shows a continuous crystalline domain between the nanocluster and the underlying nanowire which provides lithium ion diffusion pathways for lithiation and de-lithiation which enhances the capacity retention. HRTEM image also indicates the single crystalline nature of the hybrid architectures.
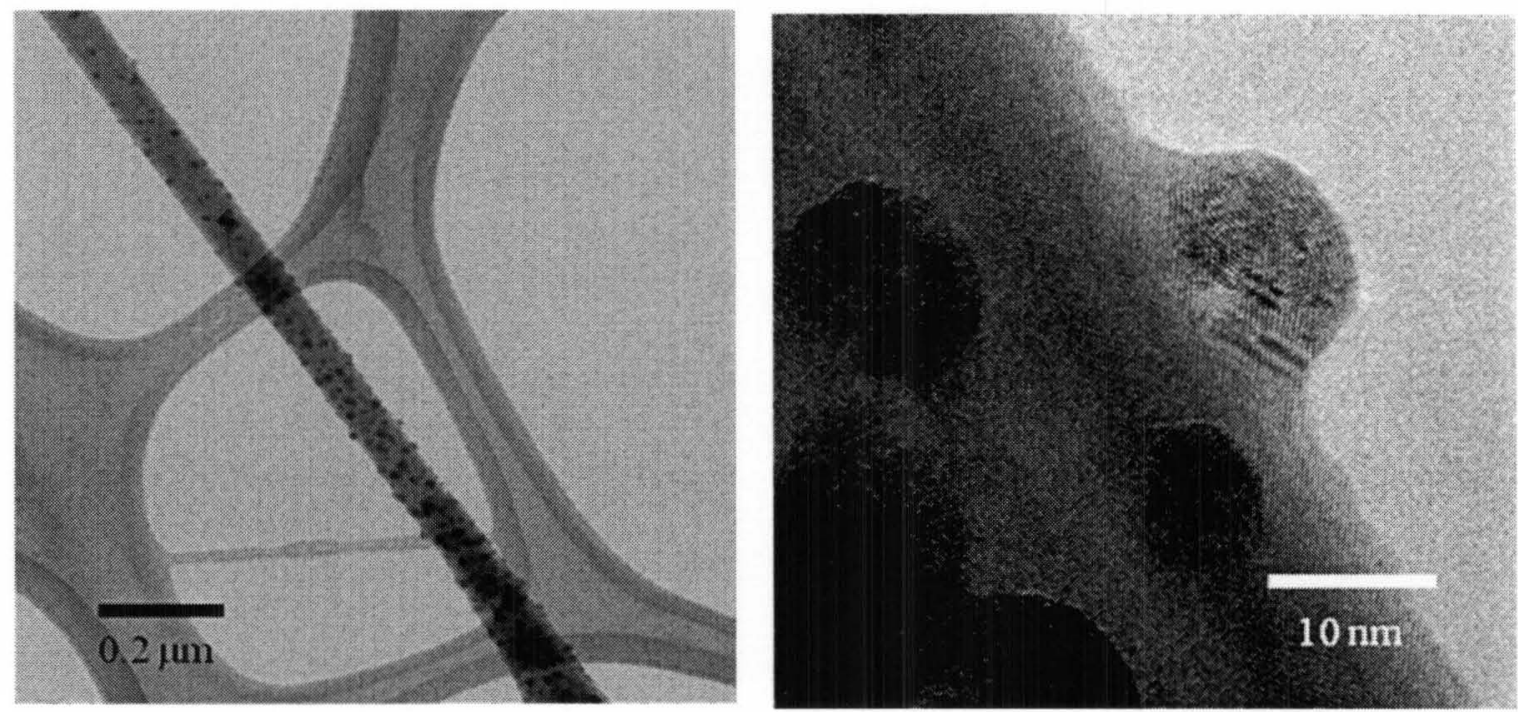

Figure 4.11: TEM images of hybrid architectures with $15 \mathrm{~nm}$ Sn nanoclusters on the nanowire periphery.

The X-ray diffraction spectrum of $\mathrm{Sn}$ nanocluster covered $\mathrm{SnO}_{2}$ nanowires shown in Figure 4.12 corresponds to the tetragonal rutile structure of bulk $\mathrm{SnO}_{2}$ (JCPDS 211250). In addition, two peaks corresponding to $\operatorname{Sn}(220)$ at $2 \theta=44^{\circ}$ and $\operatorname{Sn}(301)$ can be 
observed in the spectrum indicating the Sn formation during $\mathrm{H}_{2}$ reduction. Raman spectrum of hybrid architectures indicates the same 3 peaks as obtained for pure $\mathrm{SnO}_{2}$ nanowires as Raman spectroscopy does not detect metals.

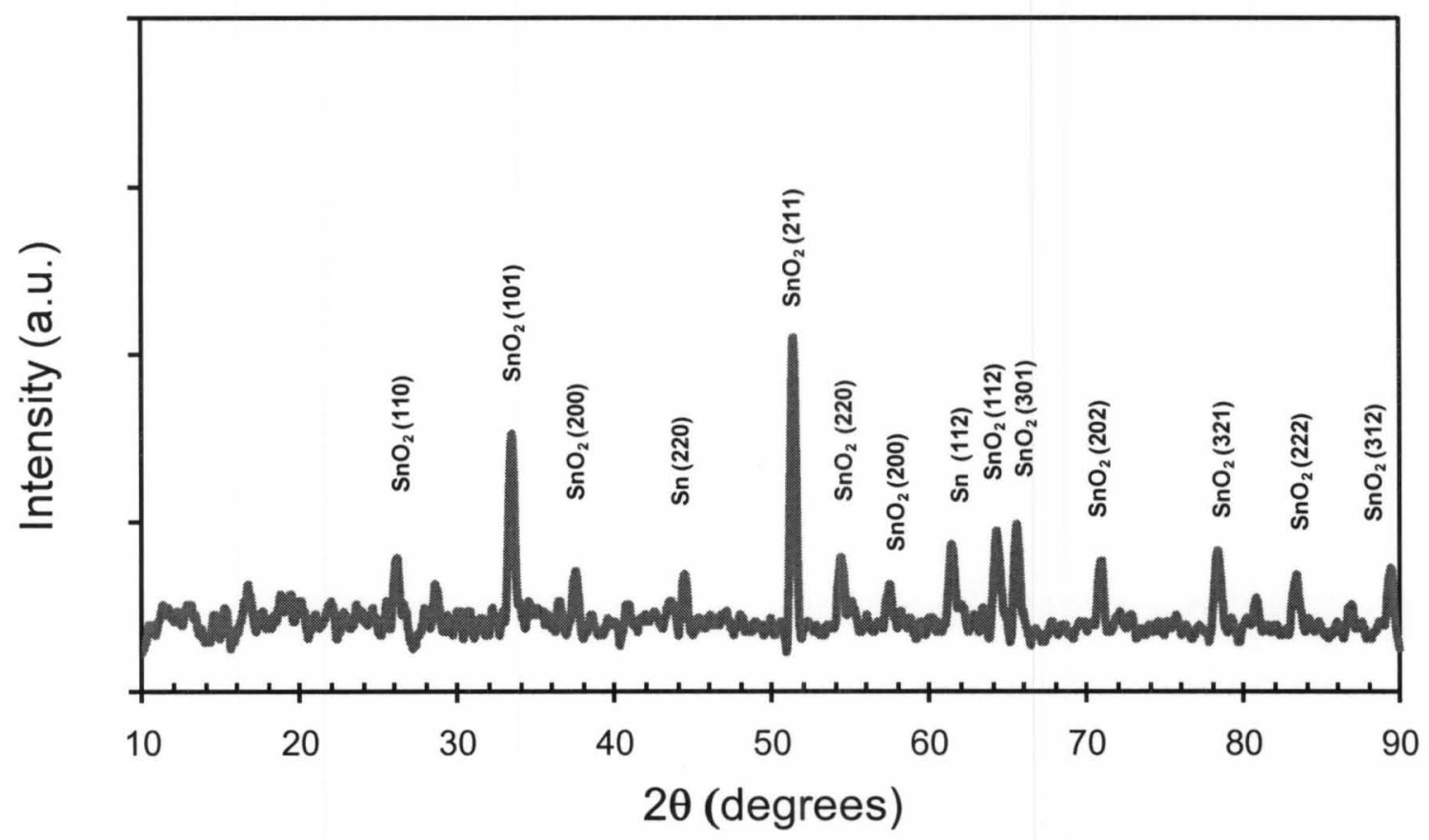

Figure 4.12: XRD spectrum of hybrid architectures clearly indicating the presence of $\mathrm{Sn}$ after $\mathrm{H}_{2}$ reduction.

\subsubsection{Enhanced electrochemical properties}

Sn-nanocluster-covered $\mathrm{SnO}_{2}$ nanowires were cycled between $0.01 \mathrm{~V}$ to $2.2 \mathrm{~V}$ with high discharge capacity of $2013 \mathrm{mAhg}^{-1}$ in the first cycle. Figure 4.13 depicts the specific capacity and the columbic efficiency at a current density of $100 \mathrm{mAhg}^{-1}$ exhibiting exceptional reversible capacity of $814 \mathrm{mAhg}^{-1}$ up to $100 \mathrm{cycles}^{138}$. This is the first time that $\mathrm{Sn} / \mathrm{SnO}_{2}$ based materials have shown mechanical stability up to 100 cycles with excellent stability. The hybrid structures show a first cycle irreversible capacity of 
$413 \mathrm{mAhg}^{-1}$. The columbic efficiency during the first cycle is $74 \%$, which is the highest reported in $\mathrm{Sn} / \mathrm{SnO}_{2}$ based systems and can be seen on secondary axis of Figure 4.13. The columbic efficiency in the second cycle is $91 \%$, third cycle is $94 \%$ and during the subsequent cycles is higher than $98 \%$ which shows the reversibility of hybrid structures. The capacity fades at $\sim 1.3 \%$ rate for the first 15 cycles and then the rate drops to $\sim 0.8 \%$ until the $100^{\text {th }}$ cycle which is the lowest reported in the $\mathrm{Sn} / \mathrm{SnO}_{2}$ systems. High discharge capacity during the first cycle is due to the $\mathrm{Li}$ intercalation with $\mathrm{SnO}_{2}$ which is followed by the alloying of $\mathrm{Sn}$ with lithium during the subsequent cycles to form a $\mathrm{Li}_{\mathrm{x}} \mathrm{Sn}$ alloy.

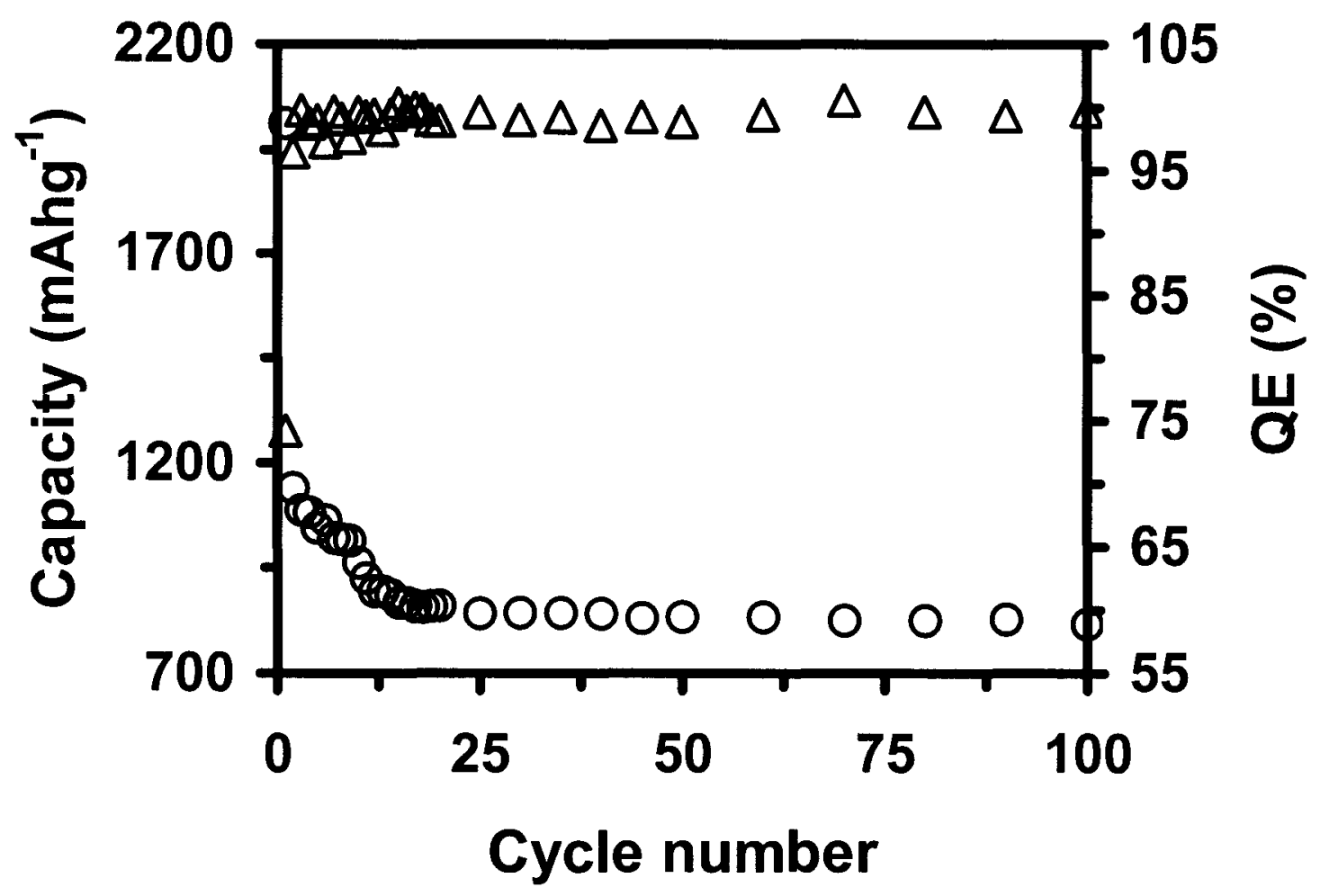

Figure 4.13: Specific capacity (in circles) and columbic efficiency (triangles) of Snnanocluster-covered $\mathrm{SnO}_{2}$ nanowires until 100 cycles indicating excellent cycling behavior. 
Figure 4.14 shows the charge-discharge curves for the first few cycles with a clear indication of plateaus below $0.5 \mathrm{~V}$ which indicates the formation of Li-Sn alloy during the discharge cycles. High capacity retention and columbic efficiency are due to (a) high available surface area to volume ratio for better lithium alloying and de-alloying. (b) single crystalline material with a continuous domain between the nanocluster and the nanowire which increases lithium diffusion inside the material.

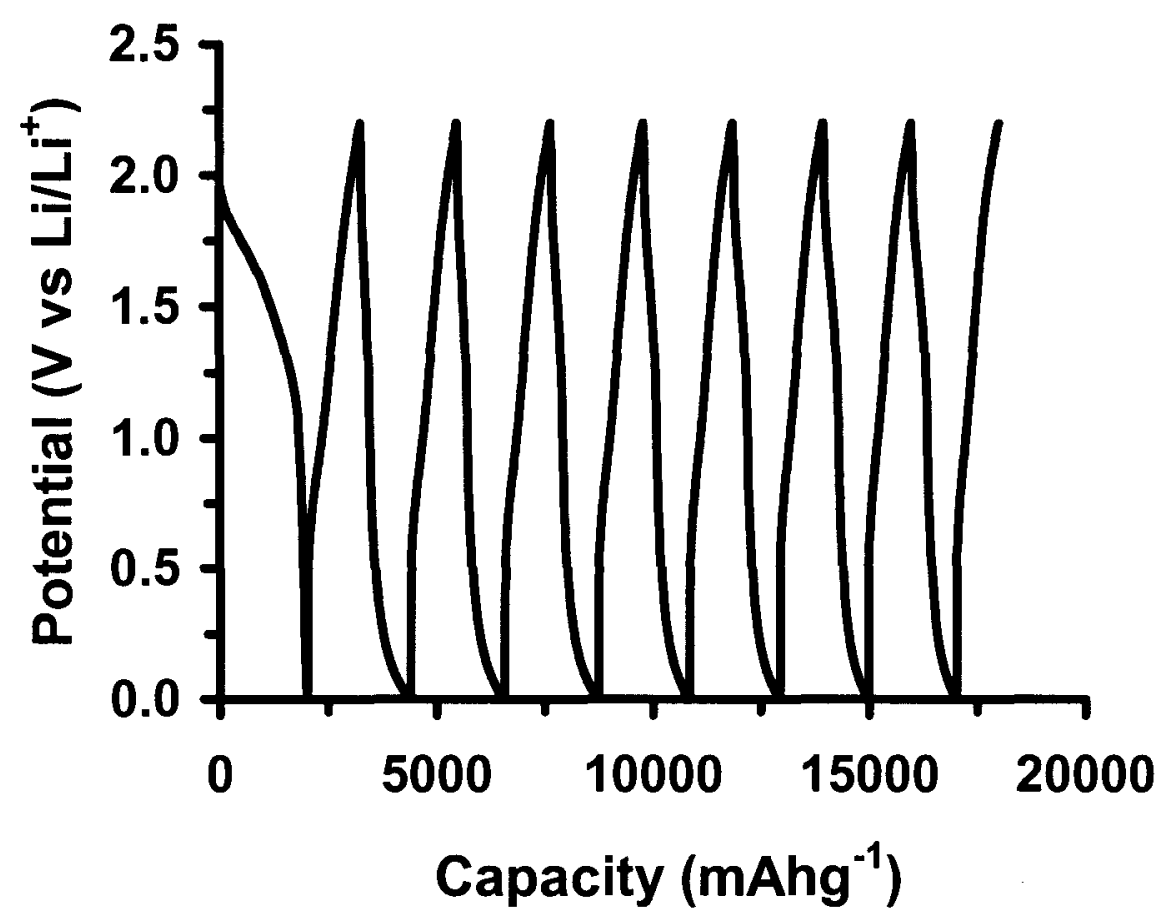

Figure 4.14: The voltage-capacity curves of the hybrid structures for first eight cycles between 0.01 and $2.2 \mathrm{~V}$ performed at a rate of $100 \mathrm{mAg}^{-1}$ and at room temperature.

Figure 4.15 shows the cyclic voltammetry curves for hybrid architectures at a scan rate of $0.1 \mathrm{mVs}^{-1}$. The peak at $1.1 \mathrm{~V}$ during the first discharge cycle indicates the decomposition of the electrolyte and formation of SEI layer leading to irreversible 
capacity. As evident from Figure 4.15, SEI is formed only during the first cycle and hence the loss of irreversible capacity from the second cycle is low.

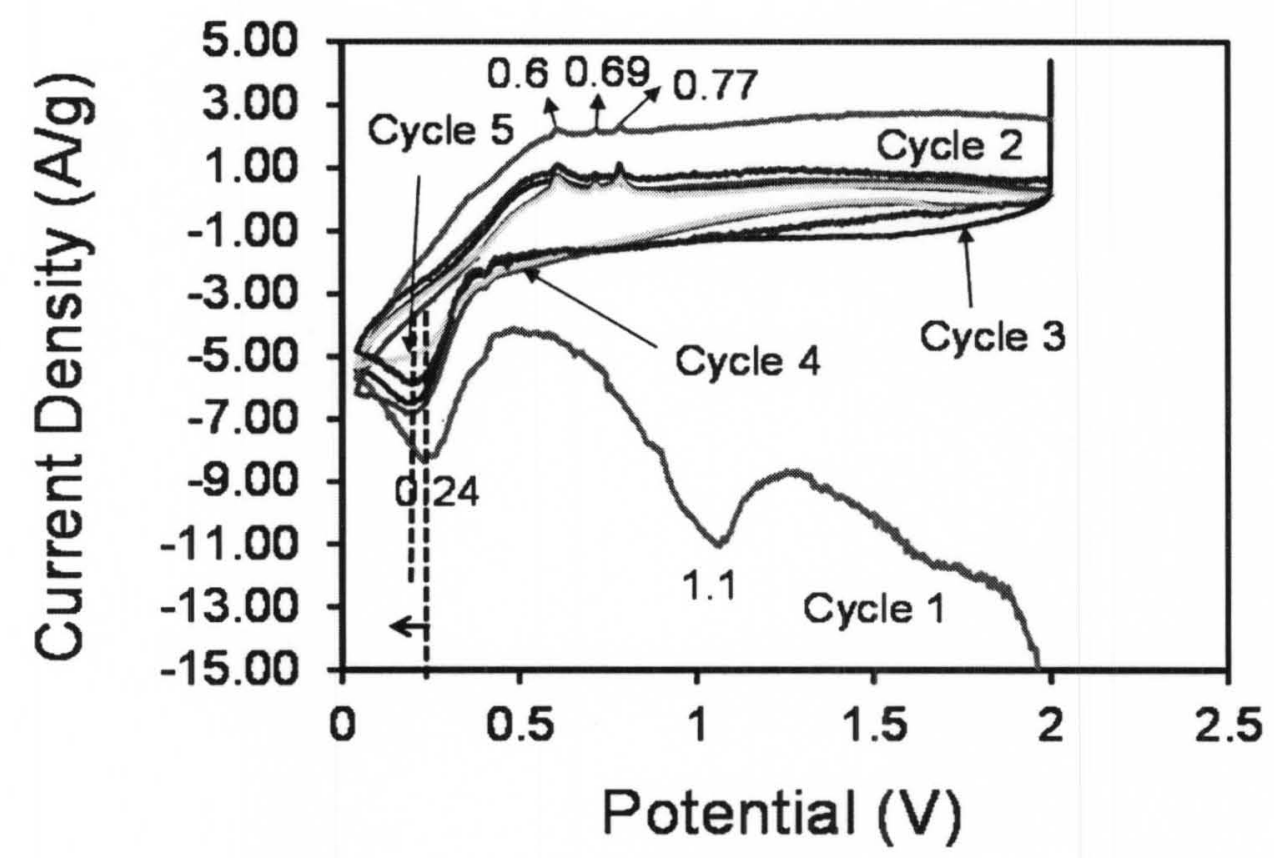

Figure 4.15: Cyclic voltammetry of hybrid nanowires for the first 5 cycles with peak positions at a rate of $0.1 \mathrm{mVs}^{-1}$.

The other peaks during the discharge cycle at $0.4 \mathrm{~V}$ and $0.24 \mathrm{~V}$ correspond to the stage wise formation of the Li-Sn alloys. Particularly, the peak at $0.24 \mathrm{~V}$ corresponds to the existence of high lithium content alloy as high as 4.4 lithium ions per tin atom. Similarly, the charge cycle shows three peaks at $0.6 \mathrm{~V}, 0.69 \mathrm{~V}$ and $0.77 \mathrm{~V}$ which correspond to different Li-Sn alloy phases and indicate a stage wise de-lithiation of lithium ions from the hybrid material. The peak currents during both the discharge and charge cycles from the second cycle are similar indicating good reversible characteristics. The excellent performance of the hybrid architectures is attributed to the fact that (a) 
material is single crystalline permitting efficient lithium ion diffusion into and out of the material (b) nanoscale Sn particles on the nanowire surface which efficiently utilize the entire area for lithiation and de-lithiation.

\subsubsection{Post lithiation characterization}

The post lithiated sample after hundred charge-discharge cycles shows hybrid architecture nanowires fairly intact with well separated Sn nanocrystals as shown in the SEM and low magnification TEM images in Figure 4.16.
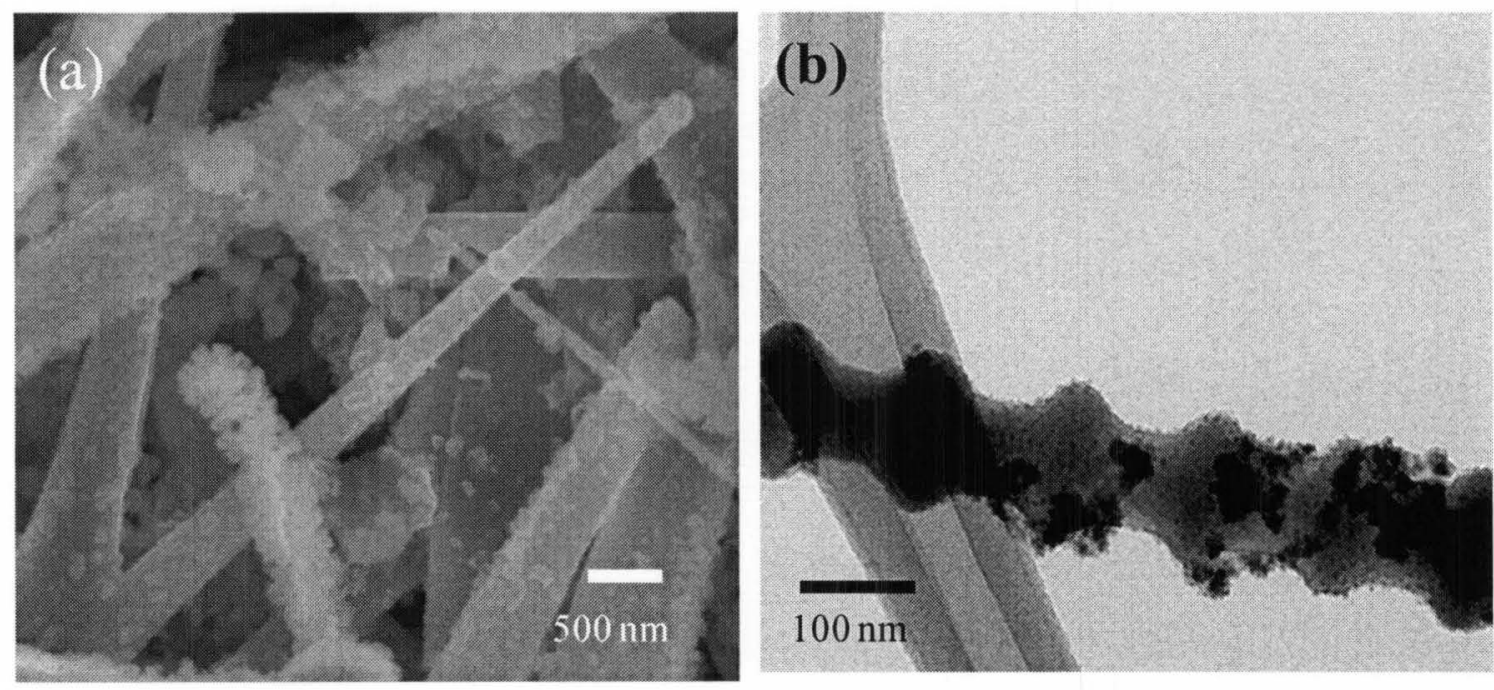

Figure 4.16: Hybrid architecture nanowires after charge-discharge cycles: a) SEM image.

b) TEM image showing the well spaced Sn nanoclusters.

However, Sn nanoclusters can be seen to be enlarged due to the volume expansion of $\mathrm{Sn}$ and $\mathrm{Sn}$ segregation on the nanowire periphery during the lithium alloying and dealloying. The average diameters of the Sn nanoclusters is $\sim 65 \mathrm{~nm}$ which corresponds to $300 \%$ volume expansion. The spacing between the nanoclusters prevents them from 
segregation with the underlying nanowires retaining their morphology and electronic conductivity which are the main reasons for the observed stability with cycling. XRD spectra obtained with the post-lithiated $\mathrm{Sn}$-nanocluster-covered $\mathrm{SnO}_{2}$ nanowire samples after being subjected to hundred charge-discharge cycles is shown in Figure 4.17.

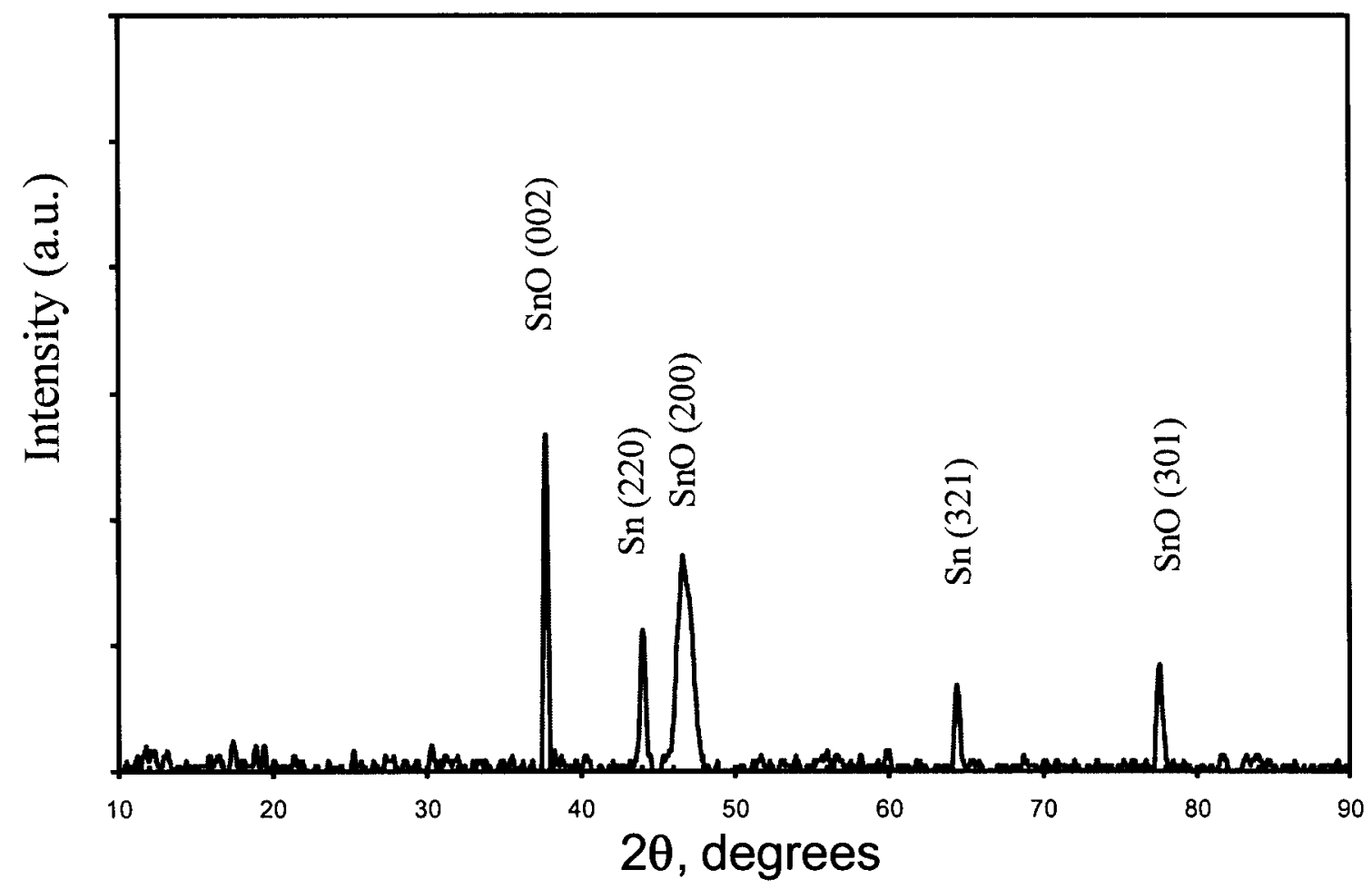

Figure 4.17: XRD spectrum of the hybrid nanowires after 100 charge-discharge cycles indicating the presence of $\mathrm{SnO}$ and $\mathrm{Sn}$.

Surprisingly, the hybrid nanowires spectrum after lithiation shows the presence of $\mathrm{SnO}(200)$, $\mathrm{SnO}(301)$ and $\mathrm{SnO}(002)$ phases in addition to Sn phases that are present before lithiation. It can be observed from the spectrum that both the $\mathrm{SnO}(301)$ and $\mathrm{SnO}$ (002) peaks shift slightly towards the lower diffraction angles indicating that the $\mathrm{SnO}$ 
phase takes part in the lithiation process. The broad $\mathrm{SnO}(200)$ peak indicates that the small crystal domains of the oxide intending to become amorphous with cycling. Capacity retention of hybrid architectures is attributed to both the $\mathrm{Sn}$ and $\mathrm{SnO}$ phases present in the material. A mechanism for the high capacity retention is proposed in the Chapter 5. The lithiation characteristics of the materials as a function of the size is also analyzed in Chapter 5 to better understand the behavior of lithium intercalation in tin and tin oxide nanostructures. In order to show that Sn addition changes the performance of these hybrid architectures, $\mathrm{Sn}$ powders were physically mixed with $\mathrm{SnO}_{2}$ nanowires to synthesize mixed metal oxides and characterized for their performance.

\subsection{Mixed metal - metal oxide materials as anodes for LIBs}

Pure phase $\mathrm{SnO}_{2}$ nanowires on lithiation and de-lithiaton undergo large volume changes which creates instability with cycling. Sn mixed with $\mathrm{SnO}_{2}$ nanowires have shown to reduce the stability problems. $\mathrm{SnO}_{2}$ reacts with $\mathrm{Li}$ to form $\mathrm{Li}_{2} \mathrm{O}$ which acts as a buffer which prevents Sn from agglomeration and hence, can reduce the problems of instability. In this case, $\mathrm{Sn}$ mixed with $\mathrm{SnO}_{2}$ nanowires has been tested as a possible anode material.

\subsubsection{Structural characteristics}

$\mathrm{SnO}_{2}$ nanowires synthesized by the methods described in the previous section were mixed with a combination of nanoscale and microscale Sn particles to obtain the mixed metal oxide in the ratio of 70:15:15. These oxides are also further oxidized in an oven at $550^{\circ} \mathrm{C}$. SEM images shown in Figure 4.18 clearly indicate the micron scale $\mathrm{Sn}$ 
particles mixed with $\mathrm{SnO}_{2}$ nanowires. The Raman spectrum of the mixed metal - metal oxides shows distinct peaks at 474, 631 and $771 \mathrm{~cm}^{-1}$ which match well with bulk $\mathrm{SnO}_{2}$ phase.

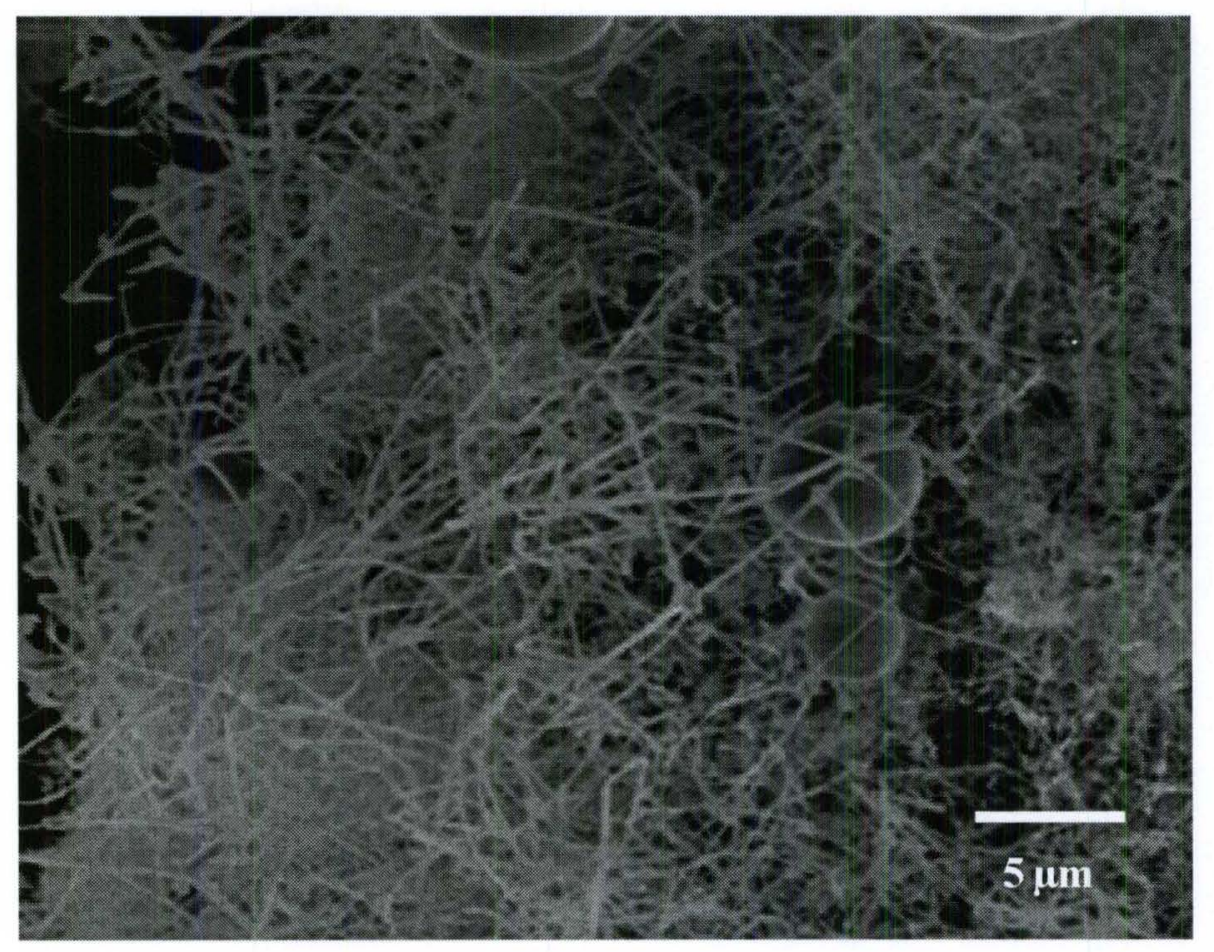

Figure 4.18: SEM images of mixed $\mathrm{Sn} / \mathrm{SnO}_{2}$ nanowires.

\subsubsection{Electrochemical characteristics}

Mixed metal - metal oxides are cycled between $0.01 \mathrm{~V}$ to $2.2 \mathrm{~V}$. These oxides show high initial capacity of $2800 \mathrm{mAhg}^{-1}$ but over 40 cycles, capacity degradation occurs and the final capacity is about $490 \mathrm{mAhg}^{-1}$. All the tests are carried out at a current density of $100 \mathrm{mAhg}^{-1}$. The 40 cycle data is presented in Figure 4.19. It can be seen here that the mixed metal - metal oxide materials outperform the pure phase $\mathrm{SnO}_{2}$ nanowire performance in terms of stability with cycling (higher capacity retention) and lower 
irreversible capacity. There is a drastic increase in the reversible capacity (250\%) and a decrease in the first cycle irreversible capacity $(20 \%)$.

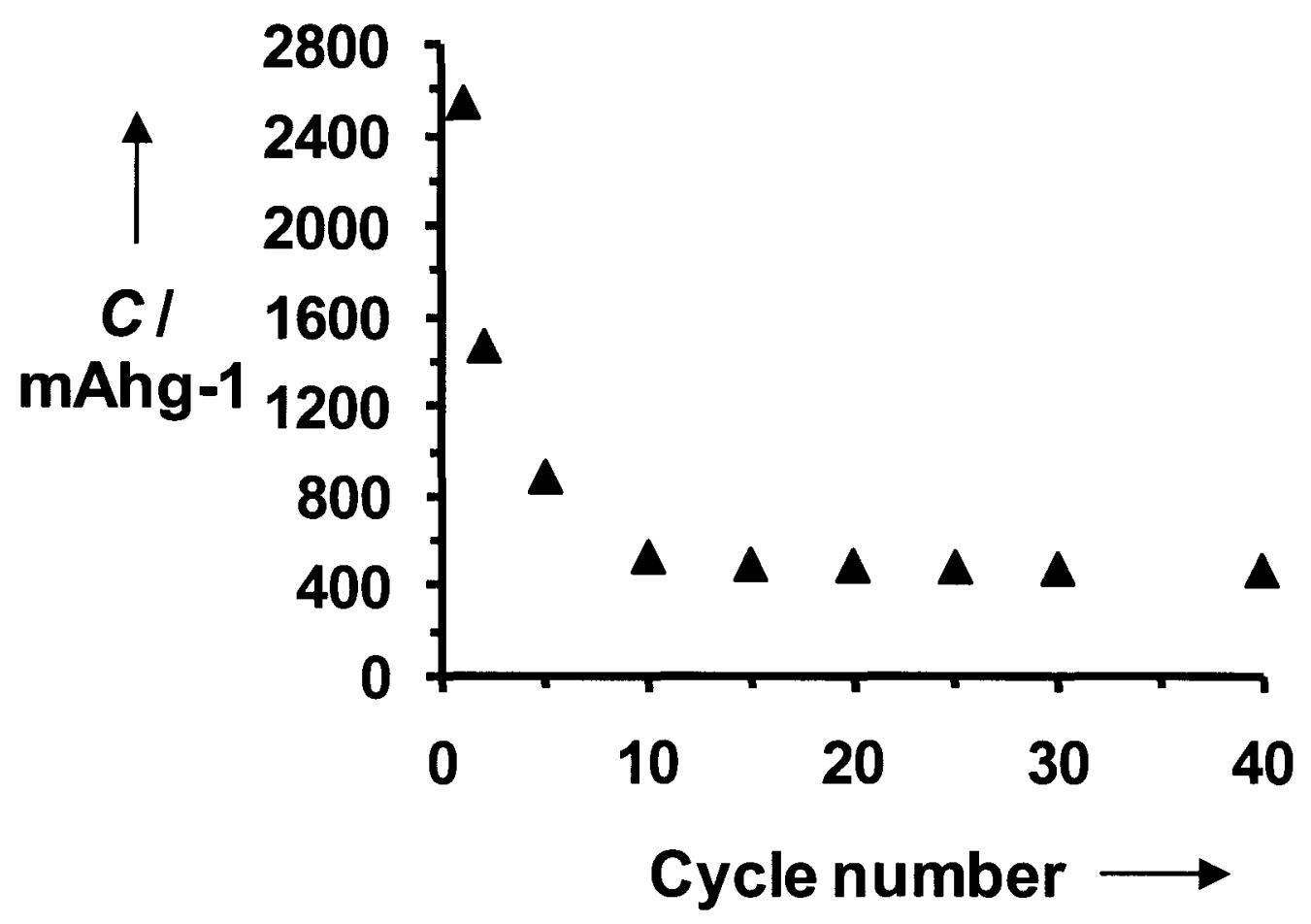

Figure 4.19: Specific capacity retention of mixed $\mathrm{Sn} / \mathrm{SnO}_{2}$ nanowires with cycling for up to 40 cycles.

The performance of the mixture is better as a result of (a) Limited oxygen content reduces the amount of lithium consumed for the formation of $\mathrm{Li}_{2} \mathrm{O}$ which explains the decrease in the first cycle irreversible capacity ${ }^{139}$. (b) $\mathrm{Li}_{2} \mathrm{O}$ formed during the first few cycles also provides a facile medium for good dispersion of the $\mathrm{Sn}$ and $\mathrm{Sn}-\mathrm{Li}$ alloy particulates which prevents them from agglomeration after large volume changes. In addition, good dispersion of the Sn particulates results in a higher available active surface area for higher capacity retention. (c) $\mathrm{Li}_{2} \mathrm{O}$ promotes faster lithium ion diffusion inside 
the material thus improving lithiation and de-lithiation ${ }^{89}$. Morimoto et al. ${ }^{140}$ have shown that milled $\mathrm{SnO}-\mathrm{B}_{2} \mathrm{O}_{3}-\mathrm{P}_{2} \mathrm{O}_{5}$ anode materials containing $\mathrm{Li}_{2} \mathrm{O}$ perform better than the ones without $\mathrm{Li}_{2} \mathrm{O}$, suggesting that $\mathrm{Li}_{2} \mathrm{O}$ facilitates lithium ion diffusion.

\subsection{Specific capacity performance of different $\mathrm{Sn} / \mathrm{SnO}_{2}$ architectures}

The performance of different architectures has been compared in Figure 4.20 demonstrating that hybrid architectures have the highest capacity retention. It is shown in the Figure that the oxidized $\mathrm{Sn} / \mathrm{SnO}_{2}$ nanowire composites show better performance $\left(\sim 600 \mathrm{mAhg}^{-1}\right)$ than the as synthesized composites of the same material. Better capacity retention for oxidized samples is attributed to the ample oxygen content which provides for better lithium diffusion characteristics and Sn dispersion, necessary for good capacity retention.

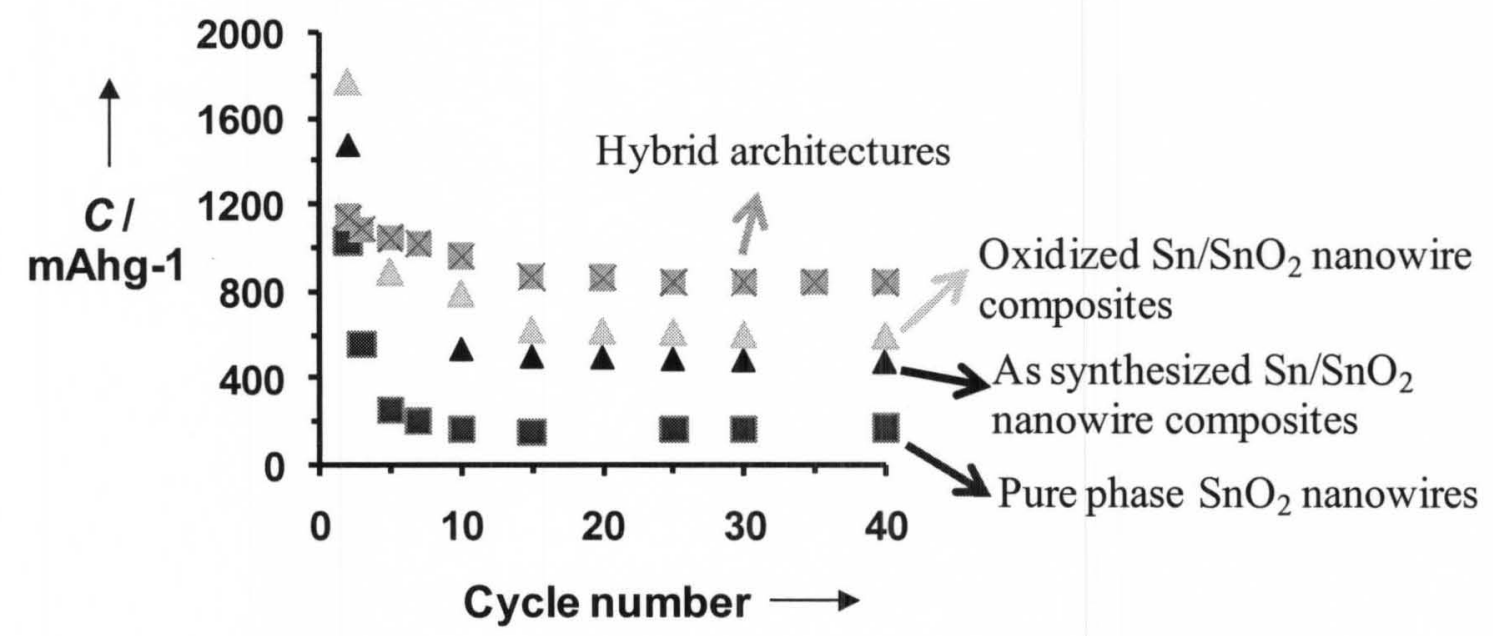

Figure 4.20: Specific capacity retention of various mixed $\mathrm{Sn} / \mathrm{SnO}_{2}$ architectures with cycling until 40 cycles. 
A systematic study with different Sn particle sizes in this case has led to the clear identification and development of hybrid architecture nanowires which have been shown to be the electrode materials of the future. It is clearly evident from the comparison that hybrid architectures outperform the 1D nanowires and material composites proving that the designed concept of hybrid architectures indeed follows the proposed hypothesis which can be handily expanded to other material systems.

\subsection{Summary}

In summary, $\mathrm{SnO}_{2}$ nanowires are evaluated as potential anode candidates for LIBs. Although, they show high initial capacity, their capacity retention with cycling is poor indicating a need to develop nanoscale architectures for improved performance. A mixed metal - metal oxide of micron sized $\mathrm{Sn}$ particles and $\mathrm{SnO}_{2}$ nanowires shows an improved performance with capacity retention of $490 \mathrm{mAhg}^{-1}$ after 40 cycles, much higher than $\sim 170 \mathrm{mAhg}^{-1}$ for $\mathrm{SnO}_{2}$ nanowires. This led to the invention of a new class of hybrid structures involving $\mathrm{SnO}_{2}$ nanowires decorated with well separated $\mathrm{Sn}$ nanoclusters on their periphery. They exhibit a reversible capacity greater than 800 $\mathrm{mAhg}^{-1}$ over 100 cycles. The capacity fading after the first few cycles is the lowest at $0.8 \%$ per cycle. Post-lithiated samples show the intact hybrid structure after 100 cycles. These hybrid structures with increased and stable capacity better relieve the stresses associated with volume changes compared to the pure phase $\mathrm{SnO}_{2}$ nanowires. The proposed new concept can be applied to other nanoscale metal oxide systems including $\mathrm{Co}_{3} \mathrm{O}_{4}\left(\sim 900 \mathrm{mAhg}^{-1}\right)$, nitride systems, and other material systems, such as $\mathrm{Si}$, leading to 
high capacity, long life Li-ion batteries and could lead to major advancements in portable power applications. 


\section{CHAPTER 5}

\section{LITHIATION/DE-LITHIATION KINETICS OF HYBRID ARCHITECTURED NANOWIRES}

\subsection{Introduction}

In the previous chapter, it was shown that pure $\mathrm{SnO}_{2}$ nanowires undergo capacity degradation which drops to $\sim 170 \mathrm{mAhg}^{-1}$ after 40 cycles at a current density of $100 \mathrm{mAg}^{-}$

1. Other reports on nanoscale $\mathrm{SnO}_{2}$ based anode materials show capacities in the range of $250 \mathrm{mAhg}^{-1}$ to $600 \mathrm{mAhg}^{-1}$ for up to 50 cycles $^{74,75,78}$. Also, it is emphasized in the previous chapter that the hybrid architectures exhibit capacity of $810 \mathrm{mAhg}^{-1}$ for up to 100 cycles at $100 \mathrm{mAg}^{-1}$ current density. The observed high capacity and retention with cycling has been attributed to the maintenance of electronic conductivity of $\mathrm{Sn}$ nanoclusters covered $\mathrm{SnO}_{2}$ nanowires through the formation of $\mathrm{SnO}$ and $\mathrm{Sn}$ phases. However, there are several fundamental questions that need to be answered: (a) does the high capacity retention result from better lithiation and de-lithiation kinetics of nanoscale tin clusters and domains within nanoscale tin covered tin oxide nanowires? (b) does the formation of solid electrolyte interface (SEI) takes place over a number of cycles? and (c) is the comparative performance of tin clusters a function of size? This chapter is aimed at understanding the lithiation/de-lithiation characteristics of hybrid architectures and answering these questions. The concept of nanocluster decorated nanowire type hybrid architectures may be the solution to address the challenges of higher capacity and 
retention with cycling of many materials systems. In fact, these structures will also be important for increasing density of packing of nanowire based materials by functionalizing them with nanoparticle based materials. So, it is important to understand the electrochemical characteristics of $\mathrm{Sn}$ nanocluster decorated $\mathrm{SnO}_{2}$ architectures to elucidate the underlying reasons for observed high capacity and retention over a number of cycles and ways to increase the rate capability.

\subsection{High capacity retention of hybrid architectures and capacity degradation of} other electrodes

\subsubsection{Mechanism of lithiation/de-lithiation of hybrid materials}

Based on the XRD spectrum shown in Figure 5.1, the stability of the Snnanocluster covered $\mathrm{SnO}_{2}$ nanowires can be explained using the following lithiation mechanism $^{138,141}$ :

$$
\begin{aligned}
& \mathrm{SnO}_{2}+2 \mathrm{Li}^{+}+2 e^{-} \rightarrow \mathrm{SnO}+\mathrm{Li}_{2} \mathrm{O} \\
& \mathrm{SnO}+2 \mathrm{Li}^{+}+2 e^{-} \leftrightarrow \mathrm{Sn}+\mathrm{Li}_{2} \mathrm{O} \\
& \mathrm{Sn}+x \mathrm{Li}^{+}+x e^{-} \leftrightarrow \mathrm{Li}_{x} \mathrm{Sn}(0 \leq x \leq 4.4)
\end{aligned}
$$

The XRD spectrum indicates the presence of $\mathrm{SnO}$ phases which is due to the reversibility of Equation 5.2. The nanoscale Sn domains on the surface can aid in the decomposition of amorphous $\mathrm{Li}_{2} \mathrm{O}$ which is irreversible under normal conditions. Nanosized metal particles of cobalt have previously shown to decompose $\mathrm{Li}_{2} \mathrm{O}$ making it feasible for reversible formation of metal oxides. This mechanism leads to the formation of SnO nanodomains from the Sn nanoparticles during the de-lithiation process, their presence clearly indicated among amorphous matrix of nanowire structures as seen in 
TEM images from Figure 5.2. SnO phase can also form because of the slightly reversible nature of the reaction itself in Equation (2) during the dealloying process; the faster kinetics for $\mathrm{SnO}$ formation leads to its formation ahead of $\mathrm{SnO}_{2}$ formation ${ }^{142,143}$.

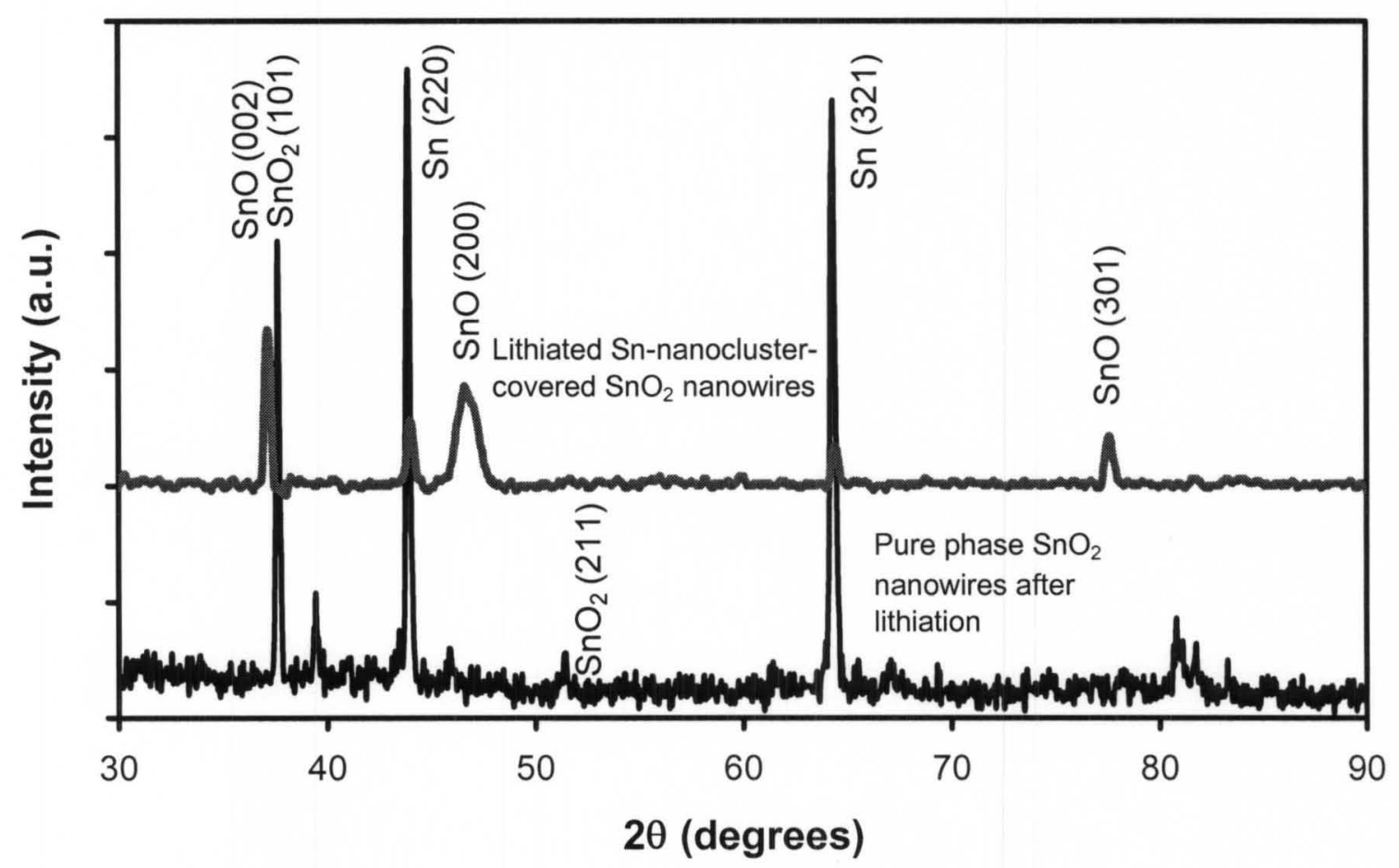

Figure 5.1: XRD spectra of both pure phase $\mathrm{SnO}_{2}$ nanowires and hybrid architectures after lithiation.

The XRD spectra of pure phase nanowires indicate the presence of Sn phase along with unreacted $\mathrm{SnO}_{2}$ nanowires. However, the hybrid nanostructures exhibit $\mathrm{Sn}$ peak from the Sn nanocrystals on the nanowire surface and $\mathrm{SnO}$ which is formed due to the reversibility of Equation 5.2. These samples also lack the presence of unreacted $\mathrm{SnO}_{2}$ indicating that most of the material has been accessed during the lithiation and delithiation processes. 

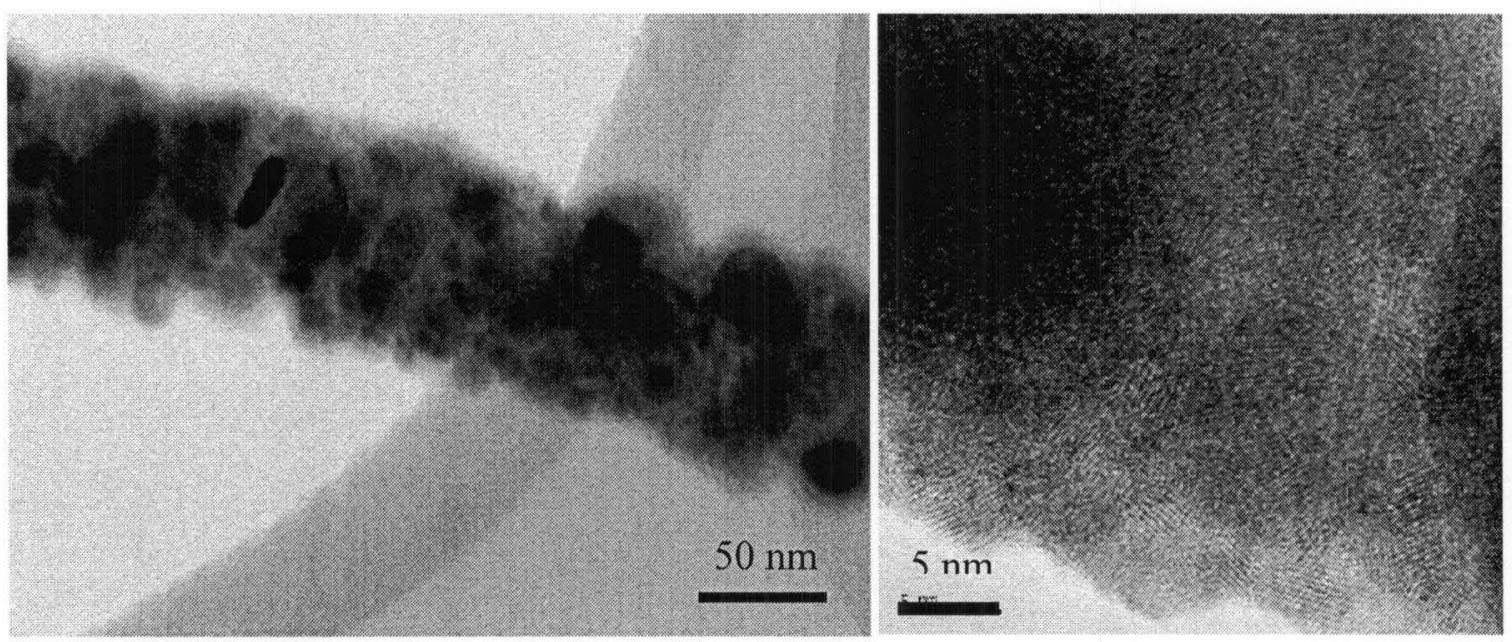

Figure 5.2: (a) Brightfield TEM image of hybrid nanowire that was subjected to hundred charge-discharge cycles. (b) High resolution image of the nanowire shown in (a). The small domains correspond to $\mathrm{SnO}$ phase.

The proposed reversibility of the hybrid nanowires is illustrated schematically in Figure 5.3.The nanowire after the lithiation process consists of a mixture of $\mathrm{Li}_{\mathrm{x}} \mathrm{Sn}, \mathrm{SnO}$ and $\mathrm{Li}_{\mathrm{y}} \mathrm{O}$, wherein (a) $\mathrm{SnO}$ promotes the electronic conductivity during the lithiation process ${ }^{89}$ (b) $\mathrm{Li}_{2} \mathrm{O}$ promotes the diffusion of lithium ions inside the material for higher amount of lithium alloying and de-alloying with $\mathrm{Sn}$ and form amorphous matrix which aids in the prevention of $\mathrm{Sn}$ agglomerates. (c) $\mathrm{Li}_{\mathrm{x}} \mathrm{Sn}$ alloy contributes to the high capacity of the material. After the de-lithiation process, the nanowire consists of $\mathrm{Sn}, \mathrm{SnO}$ and $\mathrm{Li}_{\mathrm{z}} \mathrm{O}$ wherein the amount of lithium in lithium oxide is different after the decomposition in the presence of metal nanoparticles. In the complete process, nanoscale $\mathrm{Sn}$ on the outer surface alloys with most of the lithium with minimal lithiation inside which helps to keep the nanowire structure from degradation along with amorphous lithium oxide. Hence, the material exhibits exceptional cycling stability up to 100 cycles avoiding material damage. 

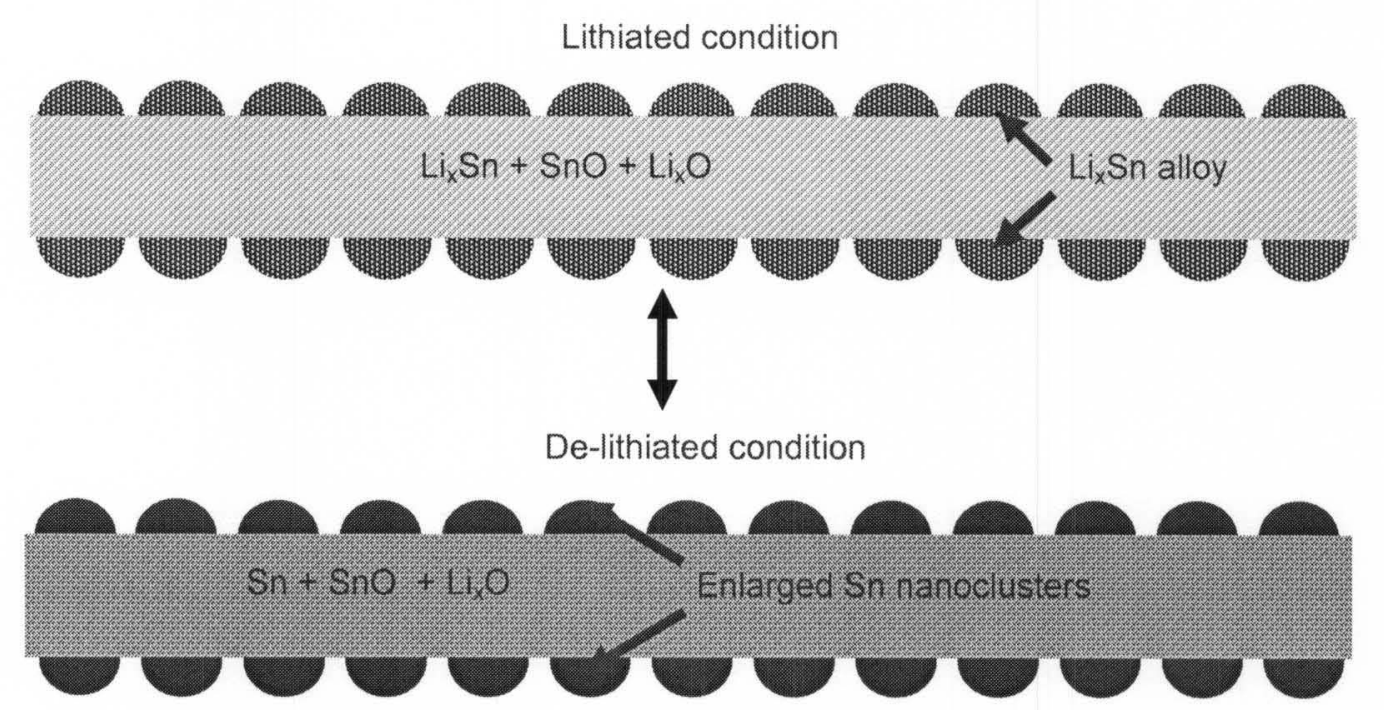

Figure 5.3: Schematic illustration of lithiation and de-lithiation processes in Snnanocluster-covered $\mathrm{SnO}_{2}$ nanowires.

\subsubsection{Comparison of lithiation/de-lithiation mechanisms of different architectures}

In this section, the science behind the interaction of $\mathrm{SnO}_{2}$ nanowires and hybrid nanowires with lithium is discussed. A typical cyclic voltammetry (CV) curve with $\mathrm{Sn} / \mathrm{SnO}_{2}$ based materials shows lithiation peaks at about $0.2 \mathrm{~V}$ and de-lithiation peaks which fall below $0.8 \mathrm{~V}$. Additional peaks in the ranges of $1.1-0.7 \mathrm{~V}$ in the lithiation scan correspond to the decomposition of the electrolytes and formation of solid electrolyte interface (SEI) ${ }^{134-136}$. CV curves of pure $\mathrm{SnO}_{2}$ nanowires in Figure 5.4 indicate peaks at $1.11 \mathrm{~V}$ and $0.8 \mathrm{~V}$ in the lithiation scan due to solvent decomposition and SEI formation leading to large irreversible capacity loss in the first cycle. The peak at $0.84 \mathrm{~V}$ is seen until $4^{\text {th }}$ cycle indicating further irreversible capacity loss with cycling. Single crystallinity of $\mathrm{SnO}_{2}$ nanowires might slow lithium diffusion and volume expansion 
leading to formation of new sites for electrolyte decomposition enabling the formation of $\mathrm{Li}_{2} \mathrm{O}$ beyond first cycle.

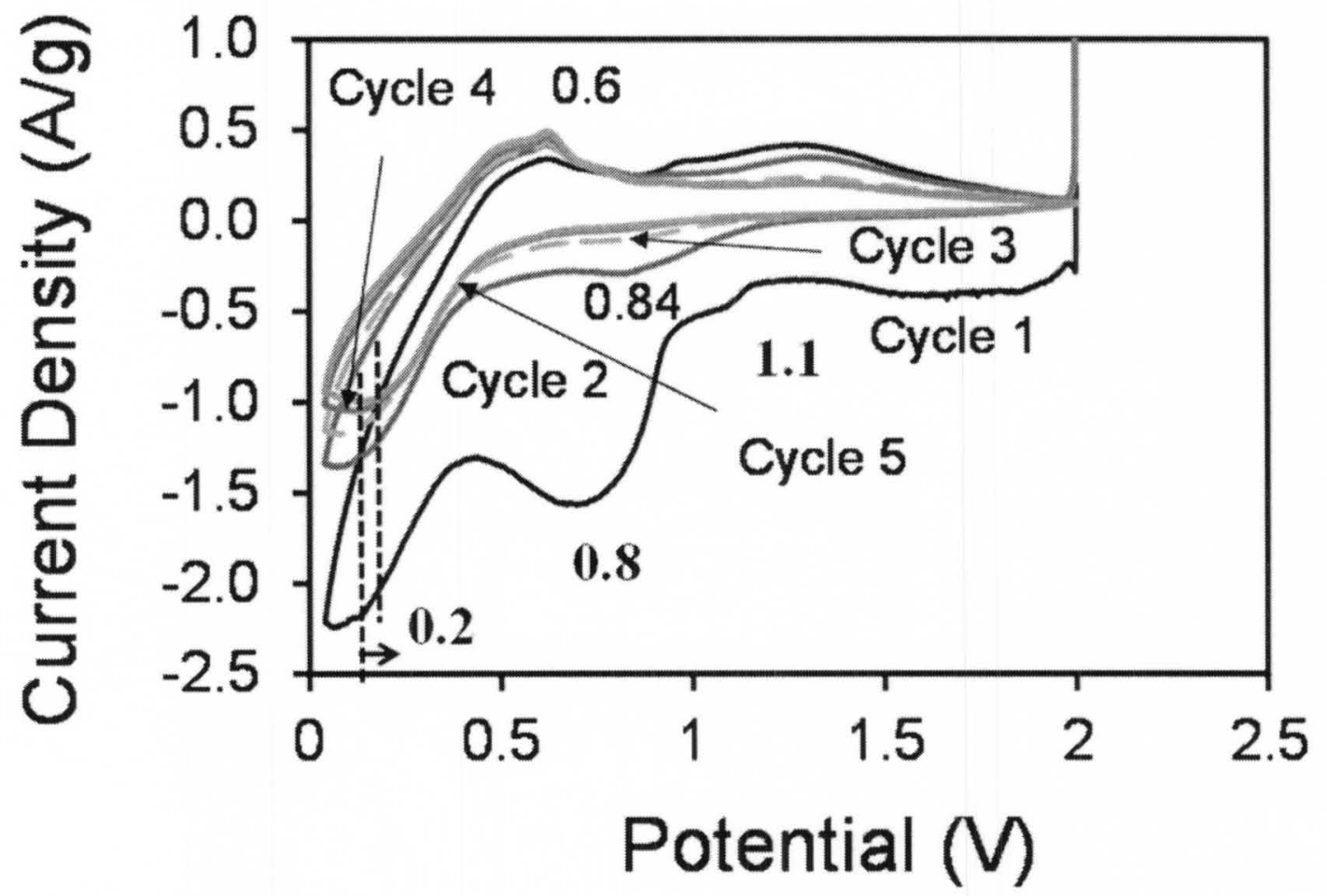

Figure 5.4: Cyclic voltammetry curves of pure phase $\mathrm{SnO}_{2}$ nanowires for the first 5 cycles with peak positions. Scan rate: $0.1 \mathrm{mVs}^{-1}$.

The peak at $0.2 \mathrm{~V}$ corresponds to the $\mathrm{Li}_{\mathrm{x}} \mathrm{Sn}$ alloy $(3.5 \leq \mathrm{x} \leq 4.4)$ giving rise to the high capacity during the first cycle ${ }^{137}$. The next 4 lithiation cycles show that alloy peak shifts from $0.22 \mathrm{~V}$ to $0.26 \mathrm{~V}$ as shown in Figure $2 \mathrm{a}$ indicating a decrease in the lithium content of the alloy. These factors lead to capacity fade with cycling in $\mathrm{SnO}_{2}$ nanowires. The charge curve shows a single peak at $0.6 \mathrm{~V}$ which corresponds to a single $\mathrm{Li}_{7} \mathrm{Sn}_{2}$ phase. This single step lithium extraction (de-lithiation) hinders the complete lithium 
removal from the material. In the case of pure $\mathrm{SnO}_{2}$ nanowires, the lithiation proceeds according to the following mechanism:

$$
\begin{gathered}
\mathrm{SnO}_{2}+4 \mathrm{Li}^{+}+4 e^{-} \rightarrow 2 \mathrm{Li}_{2} \mathrm{O}+\mathrm{Sn} \\
\mathrm{Sn}+x \mathrm{Li}^{+}+x e^{-} \leftrightarrow \mathrm{Li}_{x} \mathrm{Sn}(0 \leq x \leq 4.4)
\end{gathered}
$$

$\mathrm{Li}_{2} \mathrm{O}$ matrix formed initially as indicated in Equation 5.4 leads to disconnected $\mathrm{Sn}$ and $\mathrm{SnO}_{2}$ domains resulting in inaccessibility for lithium. Moreover, the $\mathrm{Sn}$ domains with good electronic conductivity tend to coalesce because of large volume expansions on lithiation and de-lithiation, leading to formation of large micron sized $\mathrm{Sn}$ domains and hence, decreasing capacity with cycling and with a capacity retention of only about 170 $\mathrm{mAhg}^{-1}$ after 40 cycles.

$\mathrm{Sn}$ nanocluster covered $\mathrm{SnO}_{2}$ nanowires show solvent decomposition and SEI formation peak at $1.1 \mathrm{~V}$ only during the first cycle unlike the other material systems which reduces the irreversible capacity loss with cycling. The CV curves of hybrid nanowires shown in Figure 5.5 indicate that the first and the subsequent lithiation cycles have a small peak at $0.4 \mathrm{~V}$ which corresponds to the formation of a $\mathrm{Li}_{2.5} \mathrm{Sn}$ alloy. The second peak at $0.25 \mathrm{~V}$ during the first lithiation cycle corresponds to the formation of higher lithium content alloy $(3.5 \leq \mathrm{x} \leq 4.4)$. The subsequent cycles show a shift in the peak towards lower potential shown in Figure 5.5, which in the $5^{\text {th }}$ cycle is $0.2 \mathrm{~V}$ indicating higher lithium content alloy formation. Similarly, the de-lithiation cycle shows three peaks at $0.6 \mathrm{~V}, 0.69 \mathrm{~V}$ and $0.77 \mathrm{~V}$ corresponding to $\mathrm{Li}_{3.5} \mathrm{Sn}, \mathrm{Li}_{2.5} \mathrm{Sn}$ and $\mathrm{Li}_{1.5} \mathrm{Sn}$ alloys respectively indicating a stage wise de-lithiation which results in higher amount of lithium extraction from the hybrid material. The peak currents during lithiation from the second cycle are similar indicating good reversible characteristics. The lithiation 
mechanism of the hybrid architectured nanowires is proposed and described in Section 5.2.1.

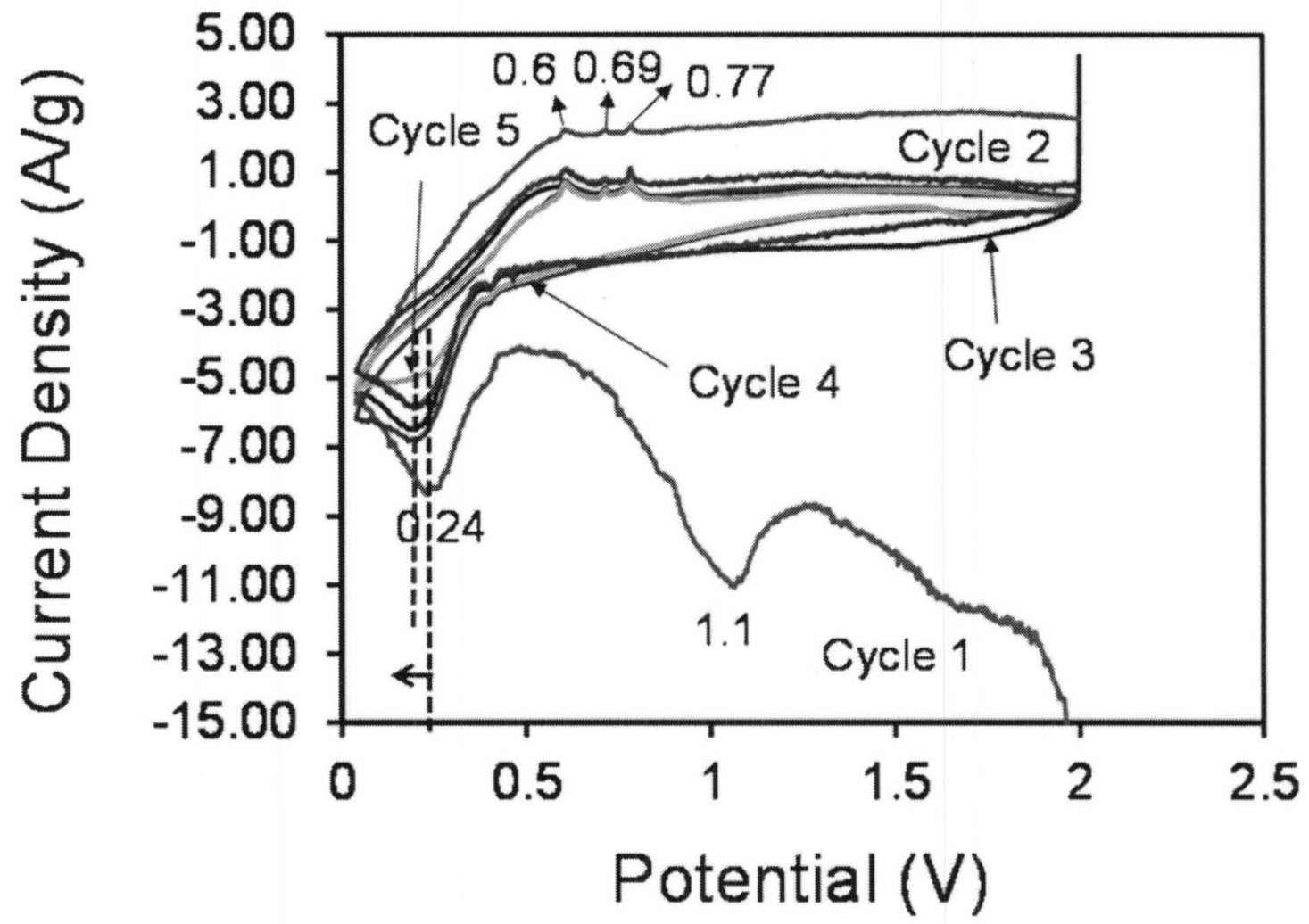

Figure 5.5: Cyclic voltammetry curves of $\mathrm{Sn}$ nanocluster covered $\mathrm{SnO}_{2}$ nanowires for the first 5 cycles with peak positions. Scan rate: $0.1 \mathrm{mVs}^{-1}$.

CVs of $\mathrm{Sn}$ nanoclusters covered $\mathrm{SnO}_{2}$ nanowires show a stage wise lithiation and de-lithiation which helps in extracting most of the lithium out of the alloy resulting in high capacity and good capacity retention with cycling. Moreover, the stage wise complete extraction of lithium helps in keeping higher available surface area even with cycling. In contrast, $\mathrm{SnO}_{2}$ nanowires show single peak de-lithiation causing poor lithium retention with cycling leading to capacity loss. Sn thin flims show single peak lithiation 
but de-lithiation over a span of 5 cycles shows decreasing lithium extraction with cycling corresponding to poor lithium retention, material degeneration and loss in capacity with cycling. In order to further strengthen the concept of stage wise de-lithiation in nanoscale $\mathrm{Sn}$, cyclic voltammetry of Sn clusters is studied as a function of the Sn cluster size. Nanoscale Sn clusters and microscale $\mathrm{Sn}$ are synthesized directly on conducting substrates and tested.

\subsubsection{Lithiation/de-lithiation characteristics as a function of particle diameter}

This section is intended to understand the lithiation and de-lithiation characteristics as a function of cluster sizes. Figures 5.6a and 5.6b are SEM images of 3-4 micron Sn clusters and $55 \mathrm{~nm}$ Sn cluster respectively.
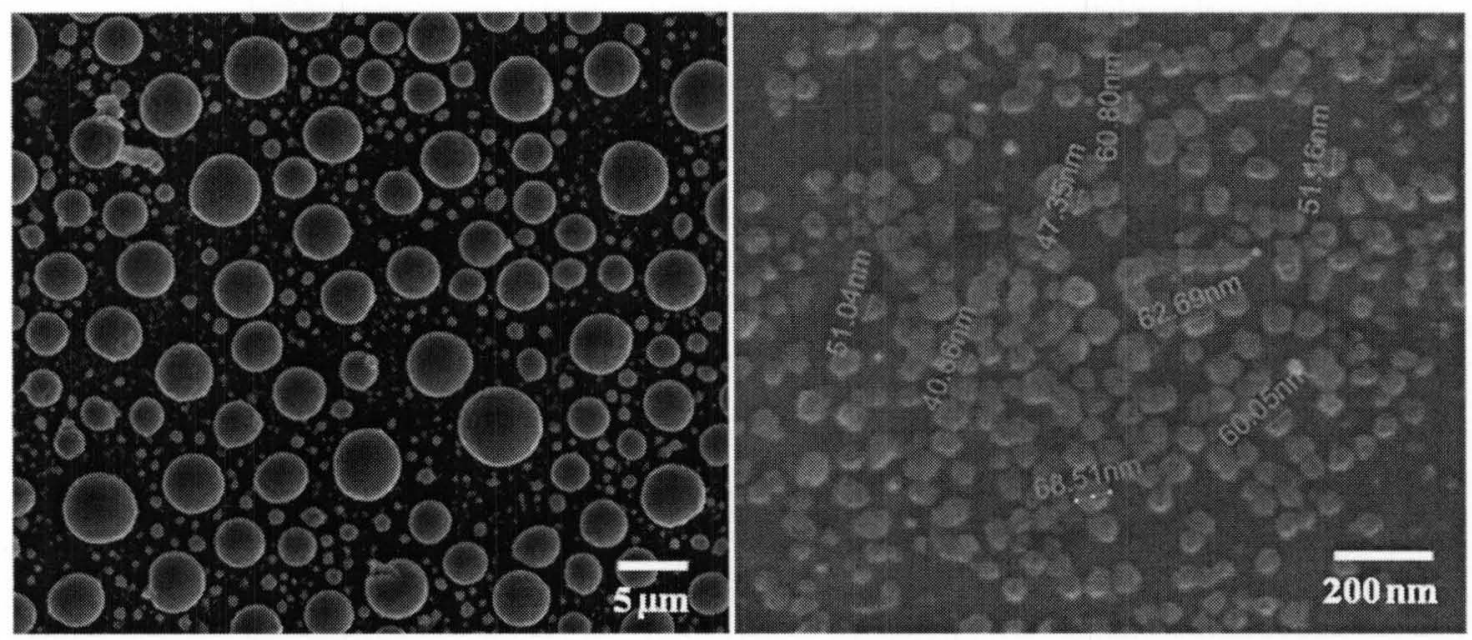

Figure 5.6: SEM images of (a) Micron scale Sn clusters/thin films synthesized directly on conducting substrates. (b) Nanoscale Sn crystals with an average diameter of $\sim 50 \mathrm{~nm}$. 
Efforts to synthesize Sn thin films have resulted in the formation of 5-10 micron Sn clusters but a continuous domain as in the case of a thin film is absent. The CV curves for both micron sized Sn samples are similar. CV data for the micron scale Sn clusters presented in Figure 5.7 show a $0.82 \mathrm{~V}$ peak during the first lithiation cycle, corresponding to the SEI formation and is also observed over the next few cycles. The lithiation peak during the first cycle at $0.25 \mathrm{~V}$ indicates the formation of high lithium content $\mathrm{Sn}$ alloy which is the case for the next 4 cycles. During the de-lithiation cycles, there are two peaks at $0.51 \mathrm{~V}$ and $0.66 \mathrm{~V}$ which correspond to Li-Sn alloys. The first cycle indicates a strong $0.51 \mathrm{~V}$ and a weak $0.66 \mathrm{~V}$ which is reversed by the $5^{\text {th }}$ cycle. The lithium content decreases as the peak positions increase. It can be asserted here that the single phase extraction in this case leads to low lithium extraction with cycling which explains the capacity loss with cycling.

$\mathrm{CV}$ analysis of Sn nanoclusters presented in Figure 5.8 shows three distinct peaks during the first discharge cycle. The peaks at $0.84 \mathrm{~V}$ and $0.62 \mathrm{~V}$ correspond to the electrolyte decomposition and SEI formation with the peak at $0.84 \mathrm{~V}$ continuing until $6^{\text {th }}$ cycle. During the discharge cycles, there is a shift in the lithiation peak from $0.2 \mathrm{~V}$ (first cycle) to $0.18 \mathrm{~V}$ (fifth cycle), both corresponding to high lithium content alloy. The initial charge cycle shows a broad peak over a range of $0.45 \mathrm{~V}$ to $1.25 \mathrm{~V}$ indicating lithium extraction from multiple alloy phases $\mathrm{Li}_{3.5} \mathrm{Sn}, \mathrm{Li}_{2.5} \mathrm{Sn}$ and $\mathrm{Li}_{1.5} \mathrm{Sn}$ which correspond to 0.6 $\mathrm{V}, 0.69 \mathrm{~V}$ and $0.77 \mathrm{~V}$, respectively. An interesting thing here is that the peak narrows with cycling spanning between $0.45 \mathrm{~V}$ to $0.85 \mathrm{~V}$ in the tenth cycle which can be attributed to nanoparticle coalescing with cycling. The smaller size particles present 
higher surface area and efficient lithiation and de-lithiation pathways inside the material, which on a micron scale is inefficient.

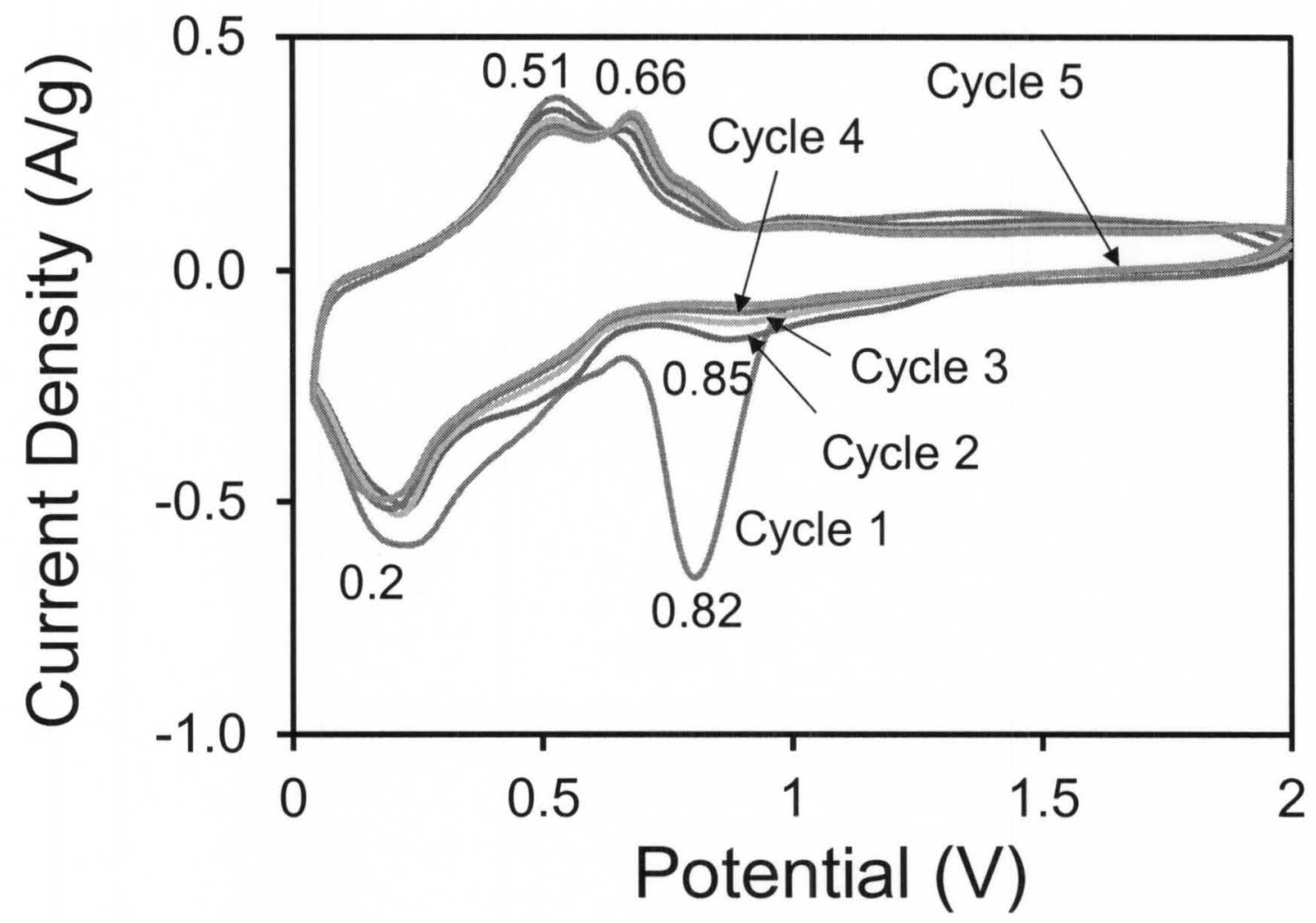

Figure 5.7: Cyclic voltammetry curves of micron scale Sn/thin films for the first 5 cycles with peak positions. Scan rate: $0.1 \mathrm{mVs}^{-1}$.

The diffusion inside a material is presented as $\tau=1^{2} / \mathrm{D}$, where $\tau$ is a characteristic time constant, 1 is the diffusion length and $\mathrm{D}$ is the diffusion coefficient. For example, $\tau$ for a particle size of micron is on the order of $0.1 \mathrm{sec}$ whereas for $100 \mathrm{~nm}$ particle is around $2.5 \mu \mathrm{sec}$. This clearly indicates that lower diffusion lengths and higher coefficients shorten the diffusion time improving the rate capability in the process. As pointed out in this study, decreasing particle size can significantly alter the lithiation characteristics than their micron scale counterparts for better performance and efficiency. 


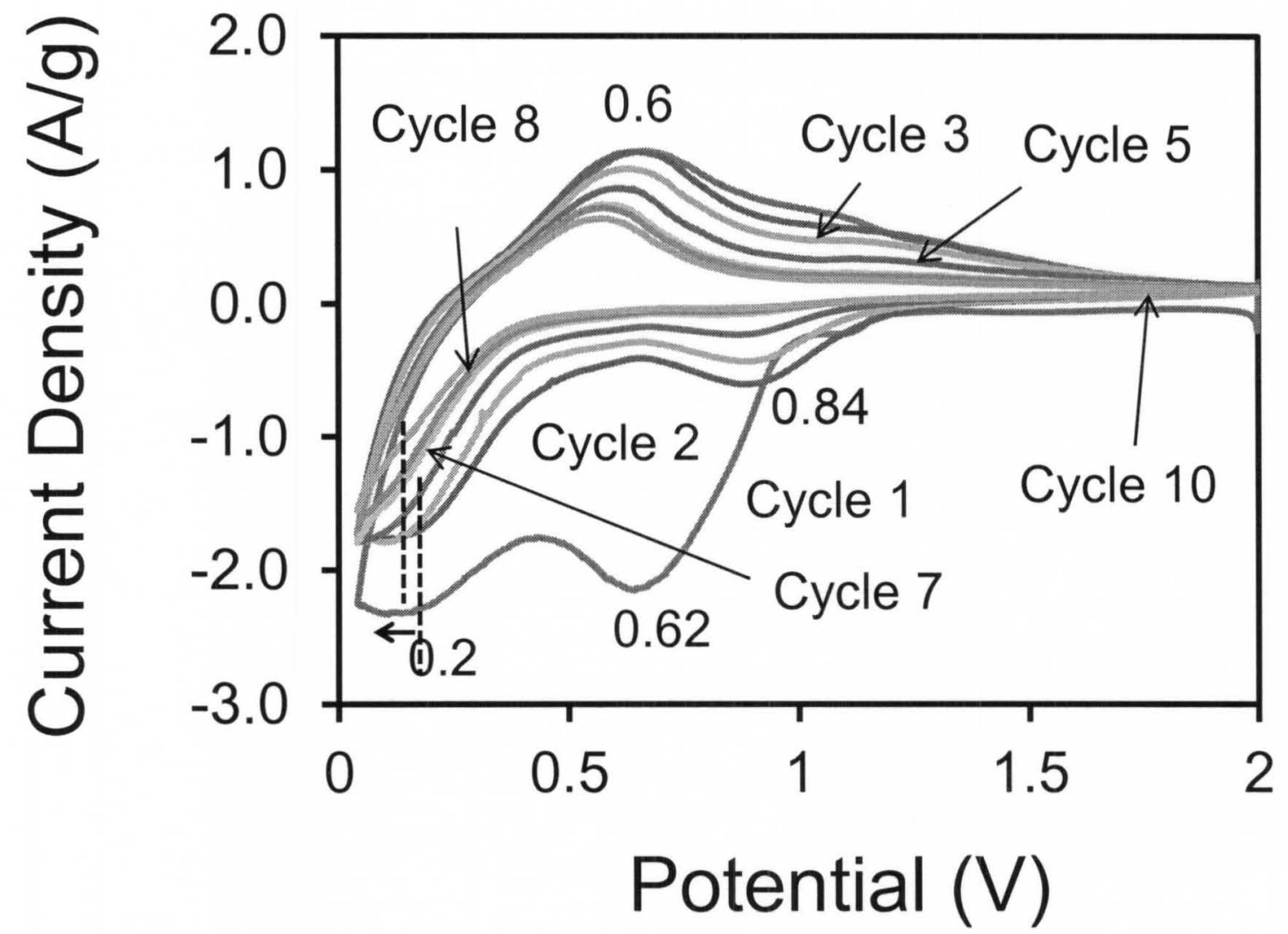

Figure 5.8: Cyclic voltammetry curves of $\mathrm{Sn}$ nanoclusters grown on conducting substrates for the first 5 cycles with peak positions. Scan rate: $0.1 \mathrm{mVs}^{-1}$.

Figure 5.9 shows the cyclic voltammetry curves of $\mathrm{Sn}$ nanoclusters grown directly on stainless steel substrates at various potential scan rates. Figure 5.10 a indicates that the potential of peak at $0.61 \mathrm{~V}$ (during first cycle) changes with the scan rate indicating that the reaction undergoes partial kinetic control unlike the micron scale particles which are diffusion controlled and hence, the potential of the peak does not change with scan rate. Figure $5.10 \mathrm{~b}$ indicates that peak potential is proportional to square root of scan rate indicating that diffusion limitation of current occurs at large polarization even in a kinetic control. 


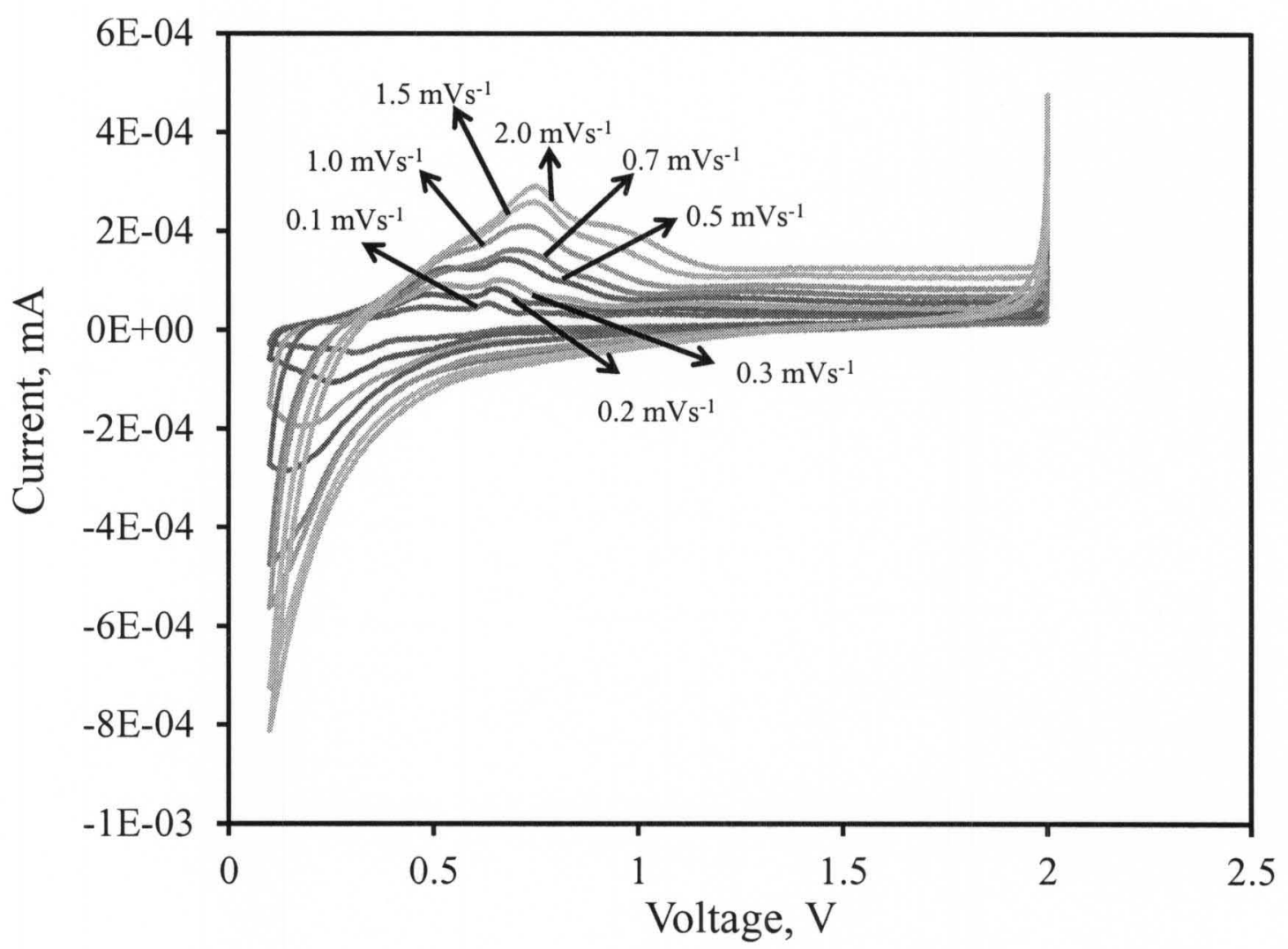

Figure 5.9: Cyclic voltammetry profiles on Sn nanoclusters grown on conducting substrates at various scan rates.
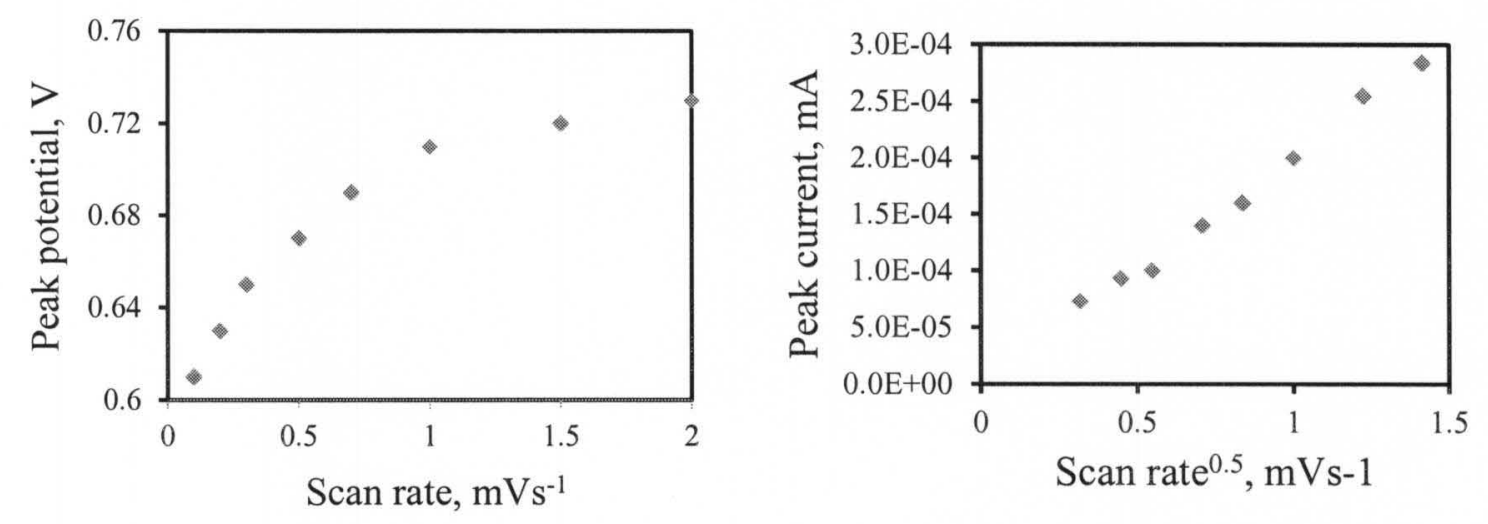

Figure 5.10: Plots of (a) scan rate vs. peak potential at $0.61 \mathrm{~V}$ peak during the first cycle. (b) scan rate ${ }^{0.5}$ vs. peak current at $0.61 \mathrm{~V}$ peak (first cycle). 


\subsection{4 $\mathrm{Li}$ concentration profile analysis}

Lithiation characteristics can be better understood by understanding the particle concentration profiles which lead to localized stresses causing particle fracture. In the case of micron sized particles, diffusion controls the Li insertion and extraction. The concentration profile for spherical particle of radius $\mathrm{R}$ and constant diffusion coefficient $\mathrm{D}$ is given by ${ }^{144-146}$ :

$$
\frac{C(r, t)-C_{0}}{C_{R}-C_{0}}=1+2 \sum_{n=1}^{\infty} \frac{-1^{n}}{n \pi z} \sin (n \pi z) e^{-n^{2} \pi^{2} \tau}
$$

where $\mathrm{z}$ (dimensionless radius) $=\mathrm{r} / \mathrm{R}$ and $\tau$ (dimensionless time constant $)=\mathrm{Dt} / \mathrm{R}^{2}$. subjected to the following conditions: Initial condition; $\mathrm{C}(\mathrm{r}, 0)=\mathrm{C}_{0}$ for all $0 \leq \mathrm{r} \leq \mathrm{R}$; At the surface: $C(R, t)=C_{R}$ for all $t \geq 0$; At the center: $C(0, t)=$ finite value for all $t \geq 0$.

The Li concentration profiles for micron sized particles are shown in Figure 5.9. Micron sized particles show diffusion gradients in the concentration profiles which correspond to a single phase $\mathrm{Li}$ extraction. For lower diffusion constants (larger particles), the concentration profile clearly indicates a build up to balance the migration of the $\mathrm{Li}$ ions. This hinders the presence of $\mathrm{Li}$ ions at the electrode/electrolyte interface which results in ineffective use of the active lithiated material and hence, leads to incomplete lithium extraction from such a region. Conversely, for nanosized particles shown in Figure 5.9, it can be seen from the profiles that the concentration profiles flatten out preventing any buildup and hence, lead to complete Li extraction in a stage wise manner. The nanoparticle insertion and extraction of $\mathrm{Li}$ are controlled by the reaction kinetics instead of the diffusion control. Particularly, for micron sized particles, $\mathrm{Li}$ 
diffusion limits the extent of de-lithiation in the material. This study complements the delithiation characteristics obtained using CV.

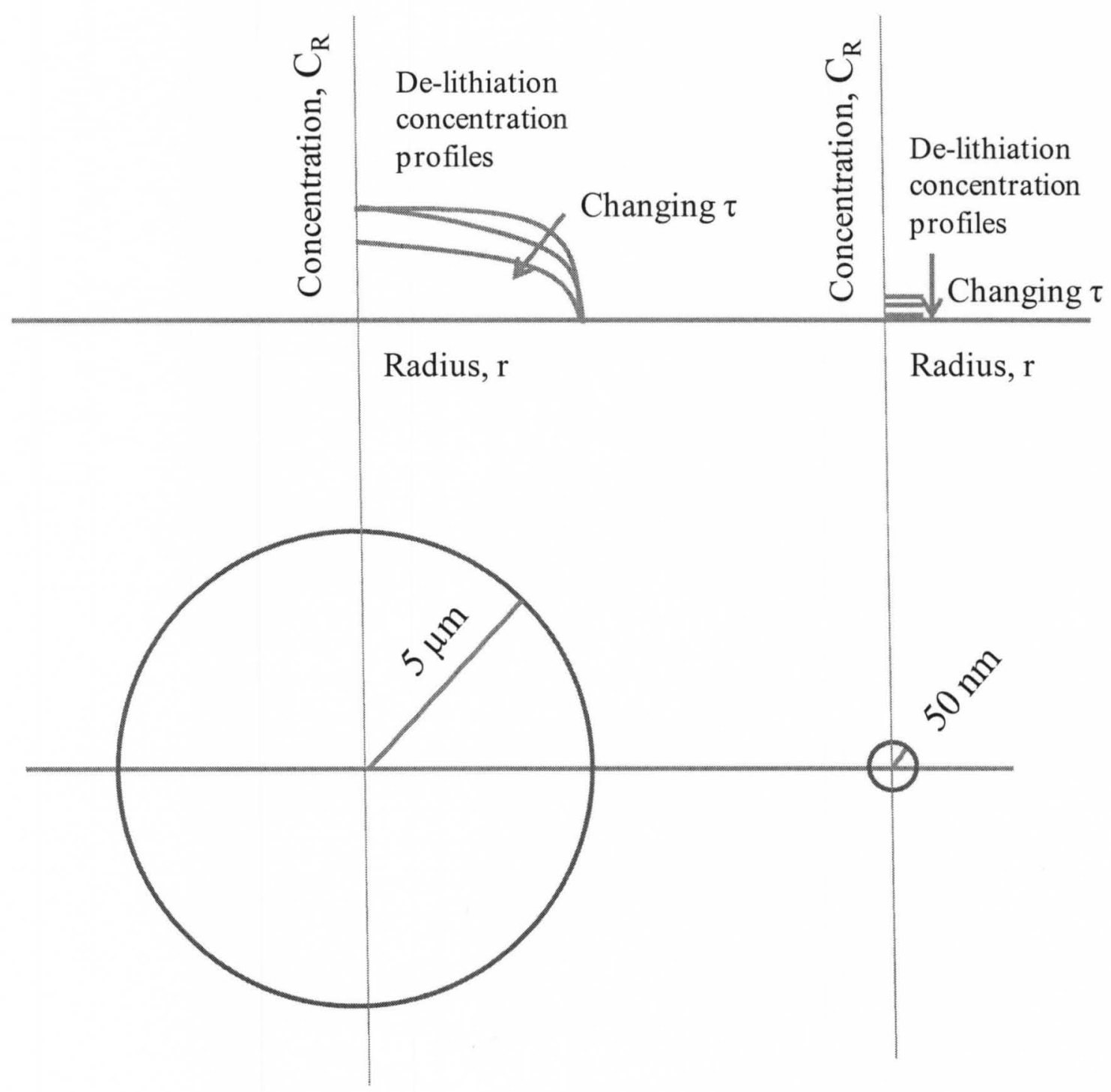

Figure 5.11: De-lithiation concentration profiles of micron sized and nanosized particles with changing time constants. The figure clearly indicates a vast difference in the profiles showing single phase de-lithiation in micron sized particles and stage-wise de-lithiation in nanoparticles. 
The study above clearly suggests that particle size has an effect on de-lithiation characteristics. The de-lithiation is controlled by diffusion until a particular size, which varies based on material characteristics after which it follows reaction kinetics.

\subsection{Rate capability of hybrid architectured nanowires}

The stage-wise de-lithiation mechanism observed in hybrid nanowires has prompted interest to look into the material capability at different current densities.

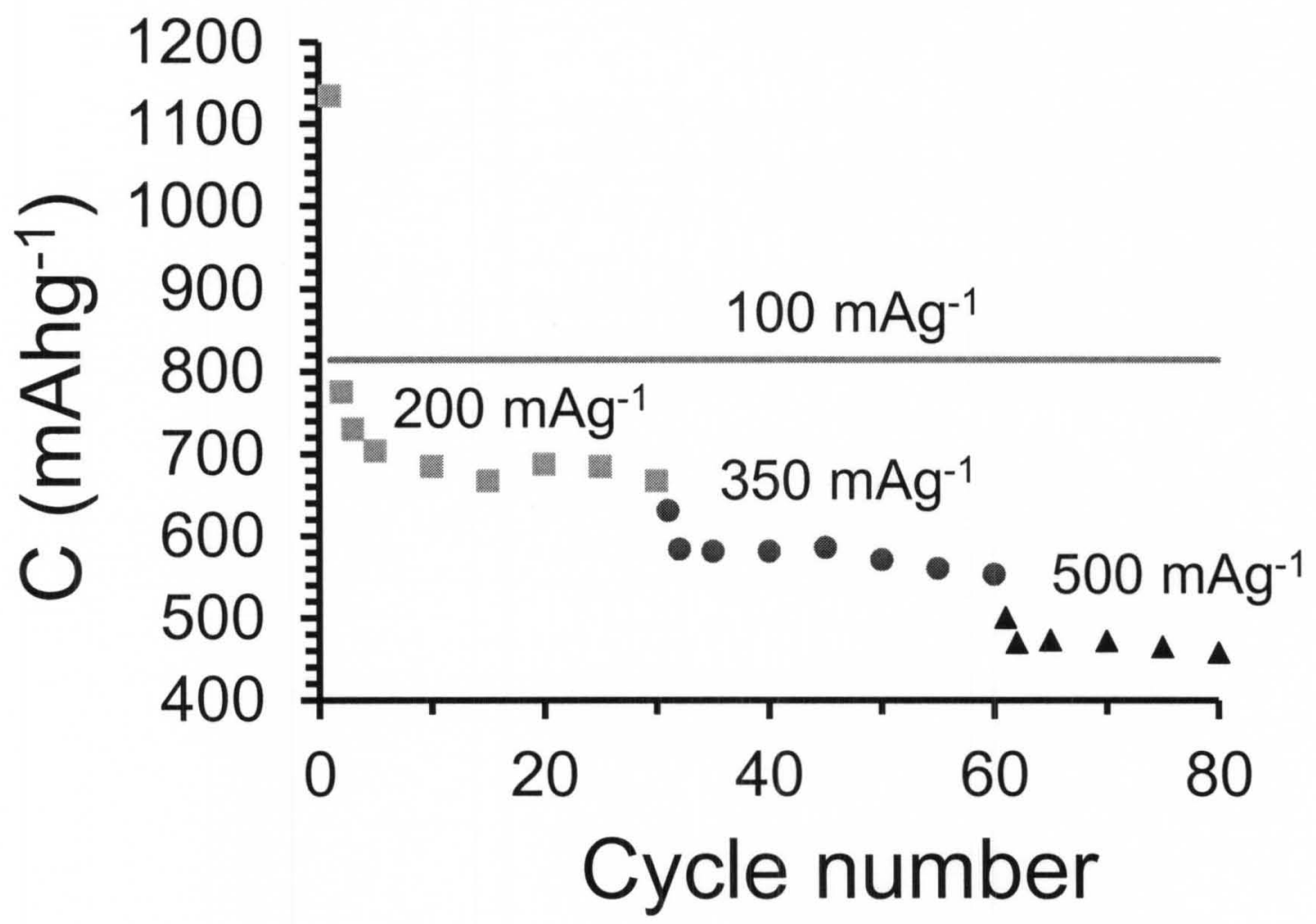

Figure 5.12: Charge-discharge cycling performance of Sn-nanocluster-covered $\mathrm{SnO}_{2}$ nanowires at various current densities.

It indicates that the material would sustain faster rates of charge and discharge. The capacity retention of $\mathrm{Sn}$ cluster covered $\mathrm{SnO}_{2}$ nanowires at current densities of 100 
$\mathrm{mAhg}^{-1}, 200 \mathrm{mAg}^{-1}, 350 \mathrm{mAg}^{-1}$ and $500 \mathrm{mAg}^{-1}$ are $814 \mathrm{mAhg}^{-1}, 667 \mathrm{mAhg}^{-1}, 553 \mathrm{mAhg}^{-}$

${ }^{1}$ and $458 \mathrm{mAhg}^{-1}$ respectively and are shown in Figure 5.10. It has to be noted that a capacity of $458 \mathrm{mAhg}^{-1}$ at $500 \mathrm{mAg}^{-1}$ is on par with the best performances reported in the literature. In addition, the capacity retention at each rate reaches a steady state within 2 cycles indicating good practical applicability. Hybrid architectured nanomaterials thus, offer high rate capability, stability with cycling with high capacity retention.

\subsection{Summary}

In summary, it can be inferred that $\mathrm{Sn}$ nanoclusters covered $\mathrm{SnO}_{2}$ nanowires show a stage-wise de-lithiation which is in complete contrast to that observed with either micron scale Sn clusters or pure phase tin oxide nanowires. Stage-wise and complete delithiation can explain the high capacity retention observed with hybrid architectures. The same complete lithiation/de-lithiation behavior has been further shown to occur only with sub-micron or nanoscale clusters of tin. In contrast, micron sized $\mathrm{Sn}$ clusters and $\mathrm{SnO}_{2}$ nanowires showed single peak de-lithiation causing lithium retention with cycling leading to material degeneration and capacity loss. The rate analysis of $\mathrm{Sn}$ nanoclusters indicates that the reaction is partially controlled by kinetics whereas the micron scale particles undergo diffusion limitation. The concentration profile calculations indicate that the micron sized particles develop a concentration gradient leading to a single stage delithiation whereas the nano sized particles indicate a stage-wise extraction leading to a complete de-lithiation of the material. Moreover, the hybrid nanostructures show good rate performance indicating better lithiation and de-lithiation characteristics. 


\section{CHAPTER 6}

\section{THIN WALLED CARBON MICROTUBES AS HIGH RATE BASE MATERIAL FOR HYBRID ARCHITECTURED ANODES}

\subsection{Introduction}

Rechargeable lithium ion (Li-ion) batteries have been demonstrated to be the best state of the art energy storage devices. In particular, highly graphitized as well as hard carbon materials have been extensively studied because of their numerous advantages like inexpensive synthesis, large scale availability, low environmental impact, good chemical stability and low potential w.r.t lithium metal. However, highly graphitized materials have low specific capacity (theoretical capacity of $372 \mathrm{mAhg}^{-1}$ corresponding to $\mathrm{LiC}_{6}$ ), limited rate capability and lithium metal plating at low voltages, where most of the specific capacity arises for these materials. Hard carbons and disordered carbons on the other hand, show higher theoretical capacity than graphite of upto $\mathrm{LiC}_{2}$ but still have limited rate capability. In order to overcome these problems, single walled and multi walled carbon nanotubes (SWCNTs and MWCNTs), carbon nanofibers (CNFs), and porous carbon materials, carbon nanobeads and carbon nanosprings have been studied as possible anode materials for Li-ion batteries. Although these materials show high initial capacities in the ranges of $700-1500 \mathrm{mAhg}^{-1}$, and in some cases good rate capability, large area synthesis of these materials and good rate capability are still the major challenges. 
Carbon microtubes (CMTs) are another morphological form of carbon with micron scale internal diameters and thin walls made of nanodomains of graphite. Thin walled CMTs were originally synthesized using gallium and the Ga filled, straight CMTs were suggested as nanothermometers ${ }^{148}$. Later, thin walled CMT synthesis with control over the conical angles and microtube diameters was demonstrated ${ }^{149,150}$. Another study showed that these CMTs can be synthesized during thermal decomposition of $\mathrm{Ga}$ precursor $^{151}$. In our present study, for the first time, we report the large area synthesis strategy of CMTs and their electrochemical characteristics. The walls of CMTs can be distinctively different from carbon nanotubes in terms of graphene planes and crystallinity. The structural characteristics suggest that CMTs with large internal diameters and nanocrystallinity within thinner walls present short diffusion lengths for $\mathrm{Li}$ ions and thus can be potentially interesting as anodes in Li-ion batteries specifically with good rates. There is no prior data or work on the Li intercalation properties of CMTs. So, here, gram quantities of CMTs are synthesized and characterized for $\mathrm{Li}$ ion intercalation capacity and performance at different charge-discharge rates. In addition, the mechanism for the high capacity, high rate capability has been proposed.

\subsection{One-dimensional carbon based materials}

\subsubsection{Carbon nanotubes (CNTs)}

Carbon nanotubes (SWNTs and MWNTs) have gained considerable interest because of their high capacity as anodes. During the charge process (electrochemical reduction), $\mathrm{Li}$ ions intercalate into carbon matrix forming lithiated carbons $\left(\mathrm{Li}_{x} \mathrm{C}_{6}\right)$. The discharge process (electrochemical oxidation) gets out the $\mathrm{Li}$ ions from the matrix. 
Commercially available MWCNTs have been used for all the testing purposes in this study. SEM images in Figure 6.1 indicate that the CNTs are $\sim 100 \mathrm{~nm}$ in diameter and are microns long.

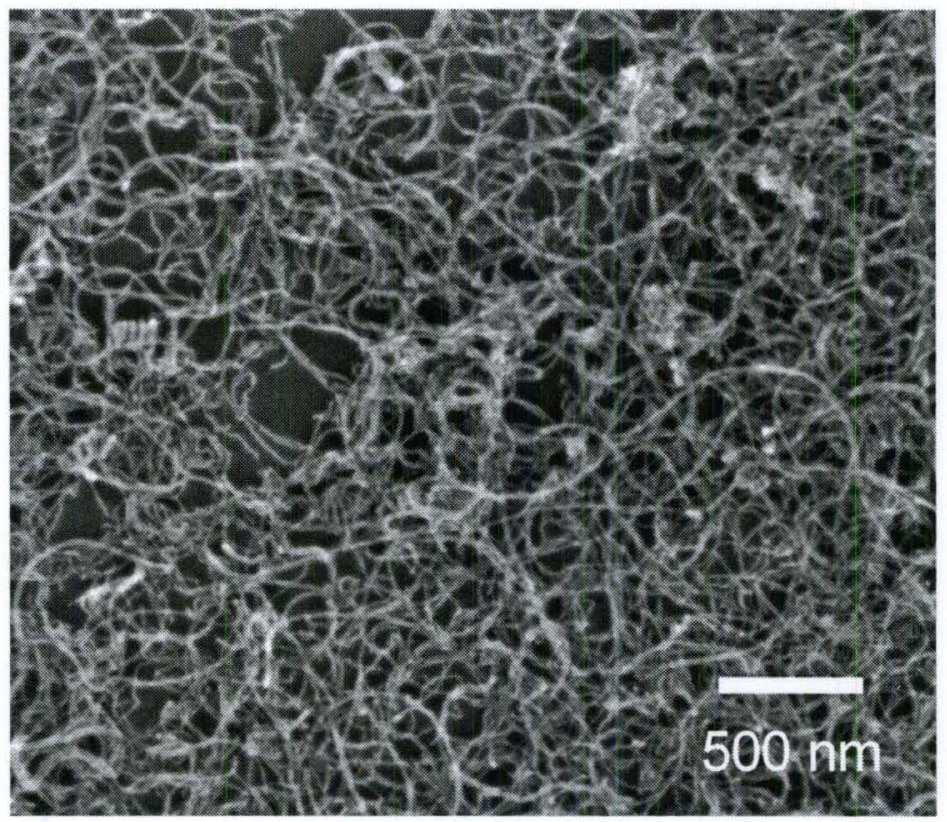

Figure 6.1: SEM image of MWCNTs with 100nm diameters and several microns length.

Figure 6.2 shows the electrochemical performance of MWCNTs with capacity retention of $\sim 250 \mathrm{mAhg}^{-1}$ after 6 cycles at a current density of $25 \mathrm{mAhg}^{-1}$. The first discharge capacity corresponds to capacity retention of $\sim 525 \mathrm{mAhg}^{-1}$ which corresponds to $1.4 \mathrm{Li}$ ions per 6 carbon atoms. There is a significant drop after the first cycle and the capacity remains fairly constant after the $2^{\text {nd }}$ cycle. The high capacity during the first cycle is attributed to the open ends of the CNTs and the defects on the walls of the MWCNTs which accommodate higher Li ion amounts per carbon atom. The significant 
drop after the first cycle is attributed to the formation of the SEI and the electrolyte decomposition during the first cycle.

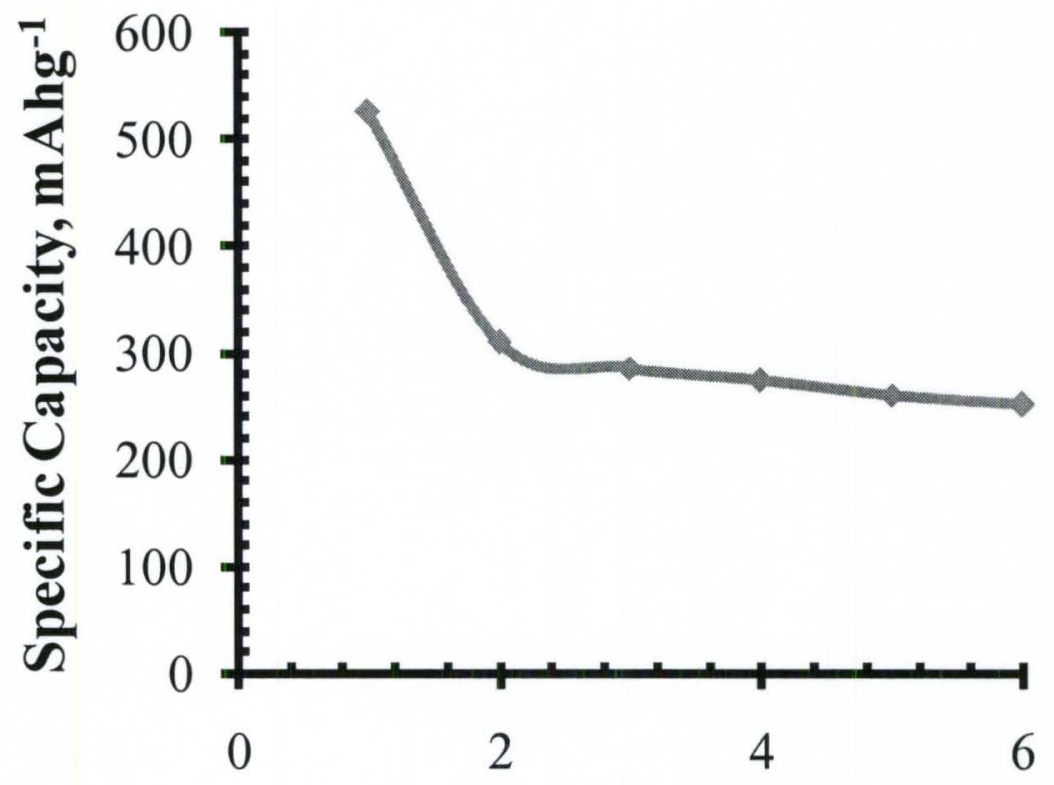

No. of Cycles

Figure 6.2: Discharge performance of pure MWCNTs at a current density of $25 \mathrm{mAhg}^{-1}$.

\subsection{Carbon microtubes}

\subsubsection{Large area synthesis of CMTs}

CMTs were synthesized on 6 inch silicon wafers in a metal organic chemical vapor deposition reactor at a substrate temperature of $700{ }^{\circ} \mathrm{C}$. The chamber pressure was set to 50 torr with a carrier gas (nitrogen) flow rate of $10 \mathrm{sccm}$ and the precursor, trimethyl gallium was set at $0{ }^{\circ} \mathrm{C}$. A 10 minute experiment covered the entirety of the silicon wafer with CMTs and the mass of the sample obtained for each run was more than 200mg. CMTs were then scraped off the silicon wafers and dispersed in aquaregia to dissolve the gallium. Aquaregia was freshly prepared by making a solution of 
concentrated nitric acid (Fisher scientific) and concentrated hydrochloric acid (Fisher scientific) in a volumetric ratio of $1: 3$. The CMTs were allowed to stand in the acid solution for an hour before removing the settled deposit of the CMTs from the solution. The CMTs were then washed with water and then dried at $200{ }^{\circ} \mathrm{C}$ for couple of hours. CMTs are formed by the decomposition of trimethyl gallium at $700^{\circ} \mathrm{C}$ giving rise to gallium metal and methyl radical species, the gallium then catalyses the growth of CMTs. CMTs are synthesized as arrays directly on silicon ( $\mathrm{Si}$ ) substrates. The CMT synthesis procedure and the structure have been described in detail elsewhere ${ }^{149-151}$. Although the present technique of using trimethyl gallium is expensive, this process presents a route of using gallium as a catalyst in the presence of methyl radical species for the inexpensive large area synthesis of CMTs.

\subsubsection{Physical characteristics of thin walled CMTs}

SEM images of as synthesized high density CMTs are presented in Figure 6.3.
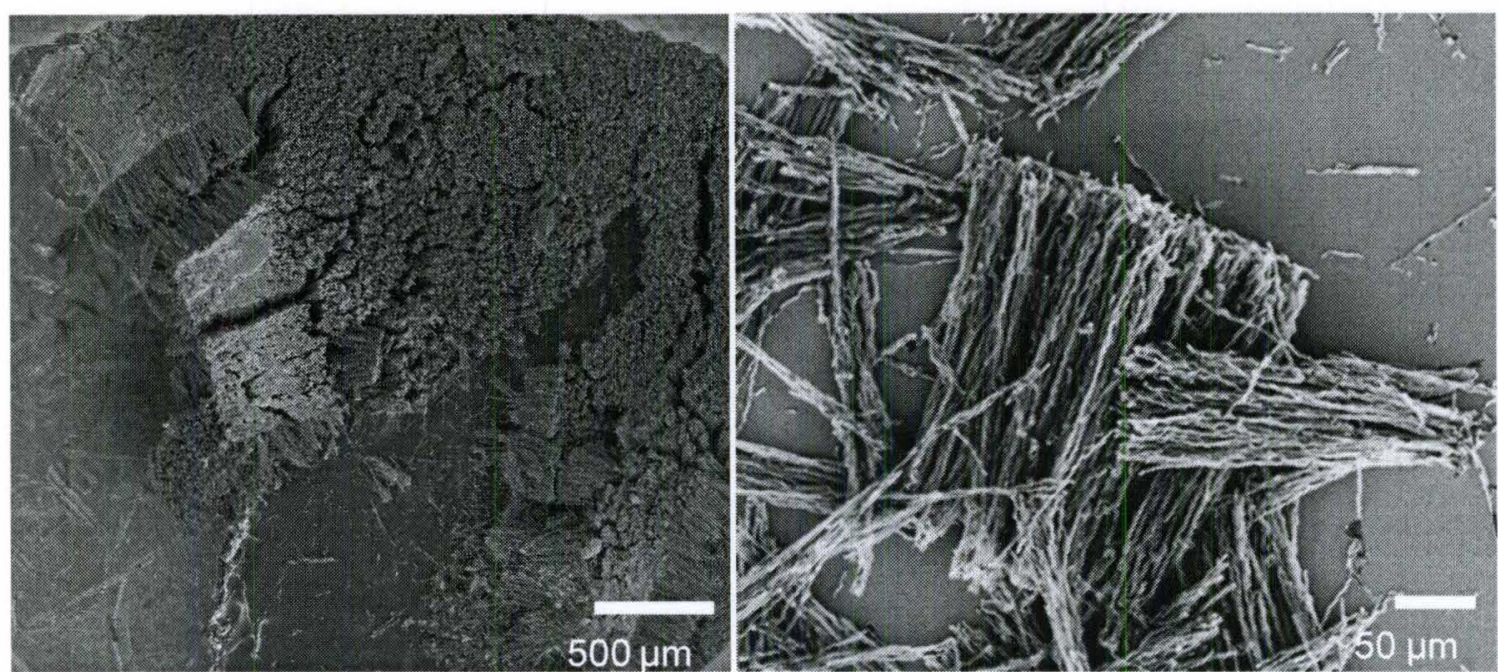

Figure 6.3: Representative images of large areas of bundled carbon microtubes as seen in SEM. 
SEM images show a large area synthesis of the material. In addition, CMTs are several microns long indicating good 1D architecture for use in LIB electrodes. The cleaned CMTs shown in the transmission electron microscopy (TEM) image in Figure 6.4 indicates that the aquaregia has dissolved off the gallium even on the inside of the CMTs. It is also evident from the figure that CMTs are $\sim 1.5 \mu \mathrm{m}$ in diameter with thin walls. An EDAX spectrum of the cleaned samples in Figure 6.5 also shows the microtubes devoid of gallium.

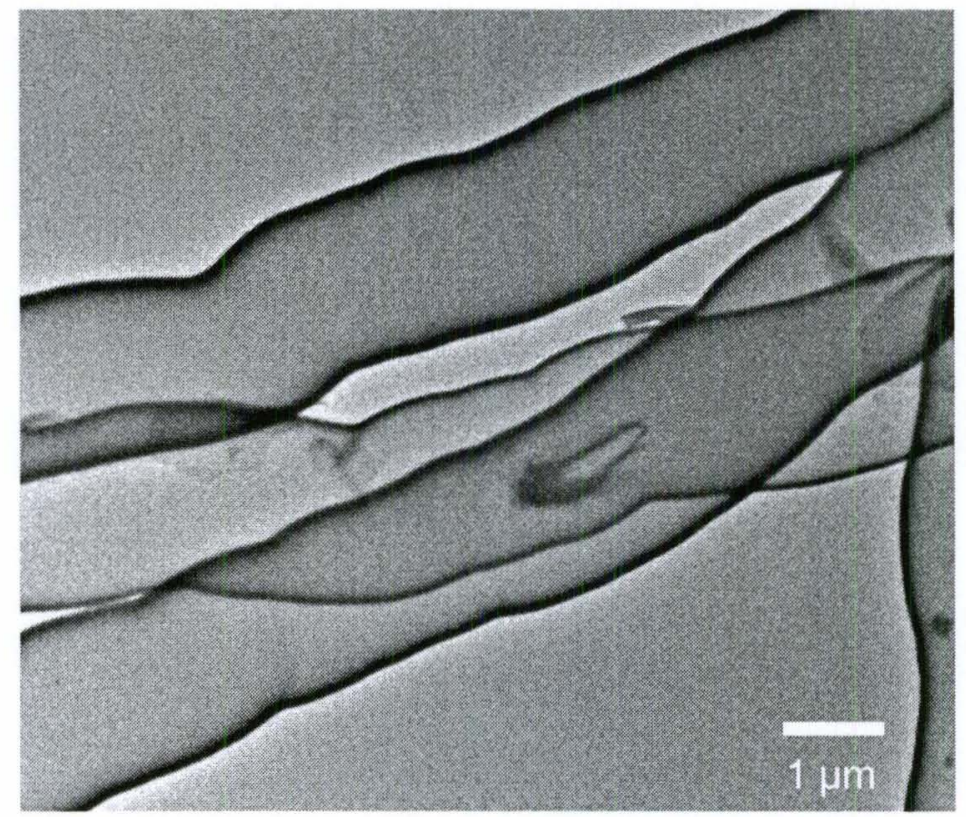

Figure 6.4: TEM image of multiple CMTs devoid of gallium.

The wall thickness as determined from the TEM image in Figure $6.6 \mathrm{a}$ is $\sim 50 \mathrm{~nm}$. Figure 6.6b is a high resolution TEM (HRTEM) image of a single CMT clearly showing the wall structure which is covered with random rotations and translations of nanodomains of graphite crystals. 


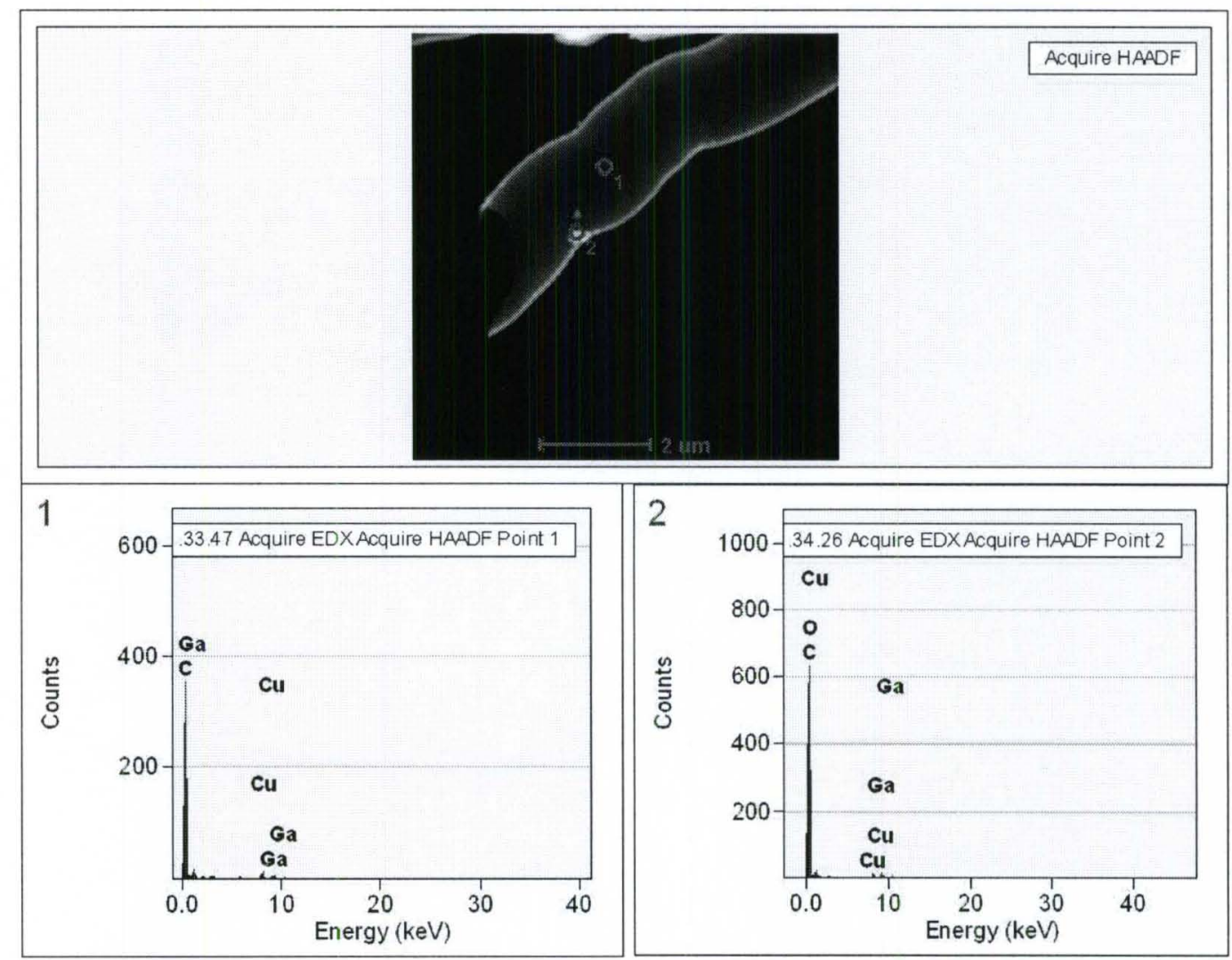

Figure 6.5: EDX spectrum of the cleaned CMTs showing the only element carbon (significantly low traces of gallium).

The inset of Figure $6.6 \mathrm{~b}$ further shows the structure of a single graphite nanodomain. The interlayer spacing $\mathrm{d}_{002}$ of the graphite nanodomains as calculated from the HRTEM image is $0.353 \AA$. It is apparent from the images that unlike the carbon nanotubes (CNTs), which are closed ends in most cases, CMTs are inherently open ended providing additional surface area inside the tube interiors. CMTs have a BrunauerEmmett-Teller (BET) surface area of $78 \mathrm{~m}^{2} / \mathrm{g}$ according to the $\mathrm{N}_{2}$ adsorption isotherm, considerably high for micron sized materials. This value is lower than the estimated 
theoretical capacity of $212 \mathrm{~m}^{2} / \mathrm{g}$ for CMTs that are $150 \mu \mathrm{m}$ long and $1.5 \mu \mathrm{m}$ in diameter, probably due to the bundling of the CMTs reducing the specific surface area.
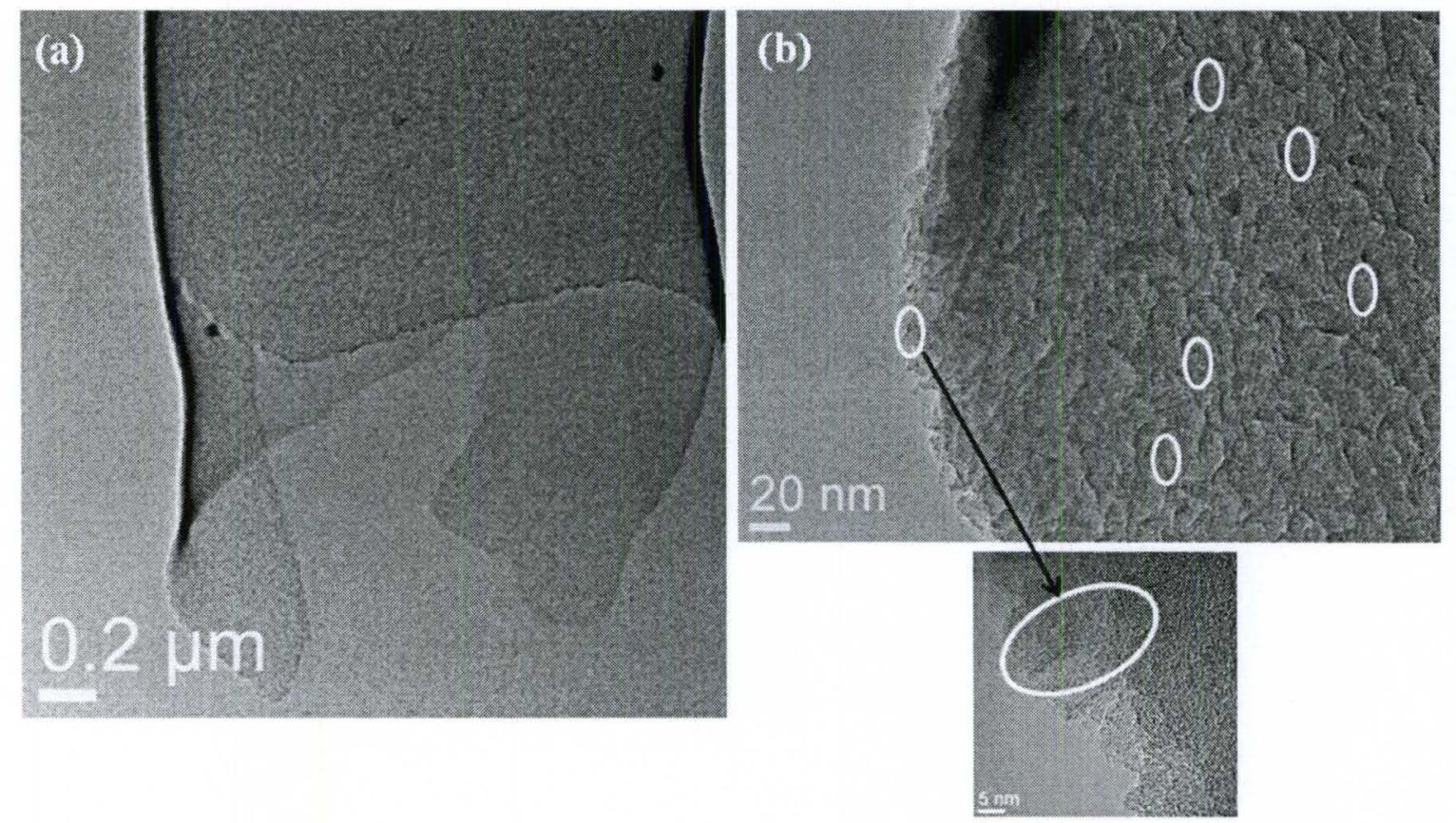

Figure 6.6: TEM images showing (a) CMT diameter and wall thickess of $\sim 50 \mathrm{~nm}$. (b) CMT wall domains in a HRTEM. Inset shows the structure of a single graphite nanodomain.).

Raman spectroscopy is one of the most widely used techniques to study the carbon atom bonding states. Raman spectrum of the as-synthesized CMTs in Figure 6.7 shows two distinct peaks at $1334 \mathrm{~cm}^{-1}$ and $1591 \mathrm{~cm}^{-1}$, corresponding to the D and G bands in the carbon structures respectively. The peak at $1591 \mathrm{~cm}^{-1}$ corresponds to the graphite $\mathrm{E}_{2 \mathrm{~g}}$ mode although the actual peak is at $1584 \mathrm{~cm}^{-1}$. The peak shift towards the higher wavenumbers has been attributed to the metal incorporation in disordered carbons which leads to breaking of the $\mathrm{sp}^{2}$ bonds with the formation of shorter carbon chains ${ }^{152}$. 
The $\mathrm{D}$ band in the spectrum corresponds to the $\mathrm{A}_{1 \mathrm{~g}}$ symmetry, which arise from the disordered carbon, in this case due to the nanocrystalline domains of graphite in the walls and the open ends of CMTs ${ }^{153,154}$. The ratio of the intensity of the D band to the $\mathrm{G}$ band indicates the degree of disordered carbon in the material, which for CMTs is 1.05 .

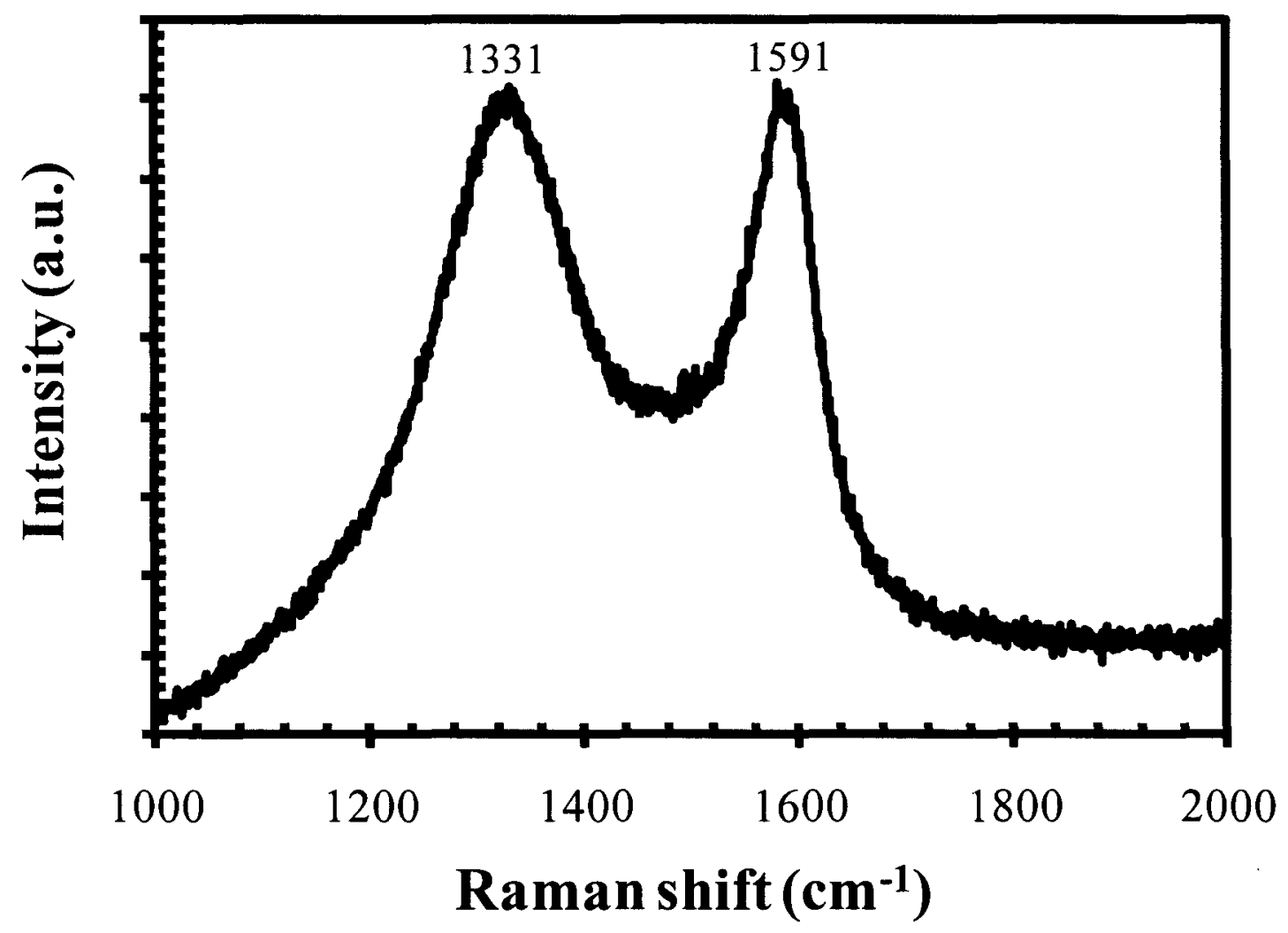

Figure 6.7: Characterization of cleaned CMTs using Raman spectroscopy for the bonding state of the carbon atoms in CMTs.

The $\mathrm{x}$-ray diffraction (XRD) spectrum of the pure CMTs is shown in Figure 6.8. The peak at $2 \theta \approx 25.2^{\circ}$ is characteristic of the $(002)$ graphite plane. The interlayer distance $\mathrm{d}_{002}$ of the CMTs is $3.53 \AA$, which is higher than the $\mathrm{d}_{002}$ of graphitic carbon $(3.35 \AA)$. The increased interlayer distance is usually the characteristic of graphite and/or 
carbon nanotubes that is ball milled for up to hundred hours. Moreover, the peak at $2 \theta \approx$ $31.6^{\circ}$ is the characteristic of ball milled graphite, which is clearly identified in other studies ${ }^{155}$. The peak around $62^{\circ}$ corresponds to graphite (103) planes which are seen in other ball milled graphite samples as well ${ }^{155}$. The XRD spectrum confirms the presence of pure CMTs devoid of gallium, with properties quite similar to ball milled graphite.

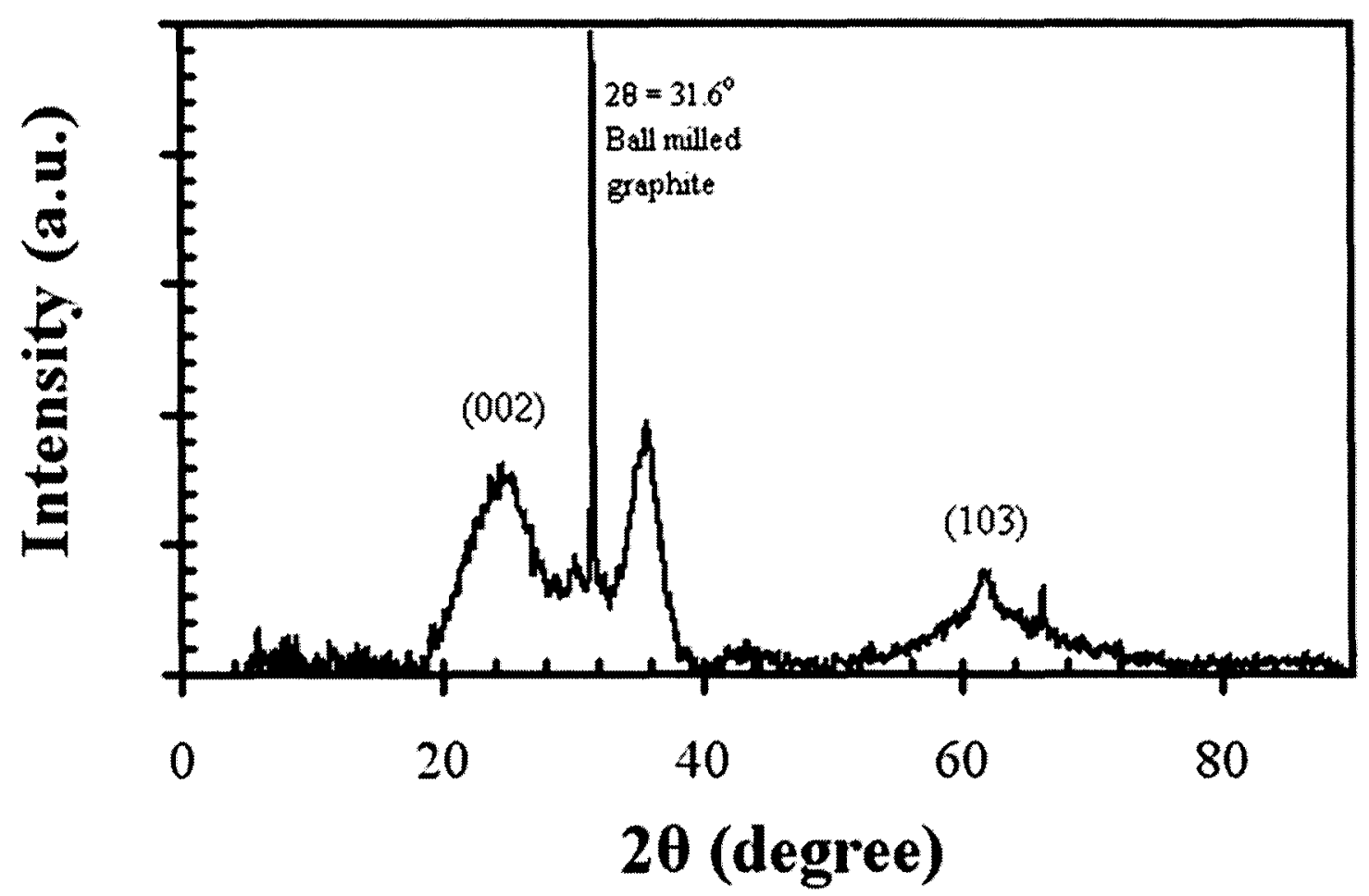

Figure 6.8: Characterization of cleaned CMTs using X-ray diffraction spectrum showing the material crystal structure and phase.

The high capacity and high rate performance of CMTs is discussed in detail in the following sections along with identifying the lithiation/de-lithiation mechanism. 


\subsubsection{Superior electrochemical characteristics of CMTs}

\subsubsection{High capacity performance}

CMTs are tested as potential anodes for lithium ion batteries. The specific capacity with cycling of CMTs is presented in Figure 6.9.

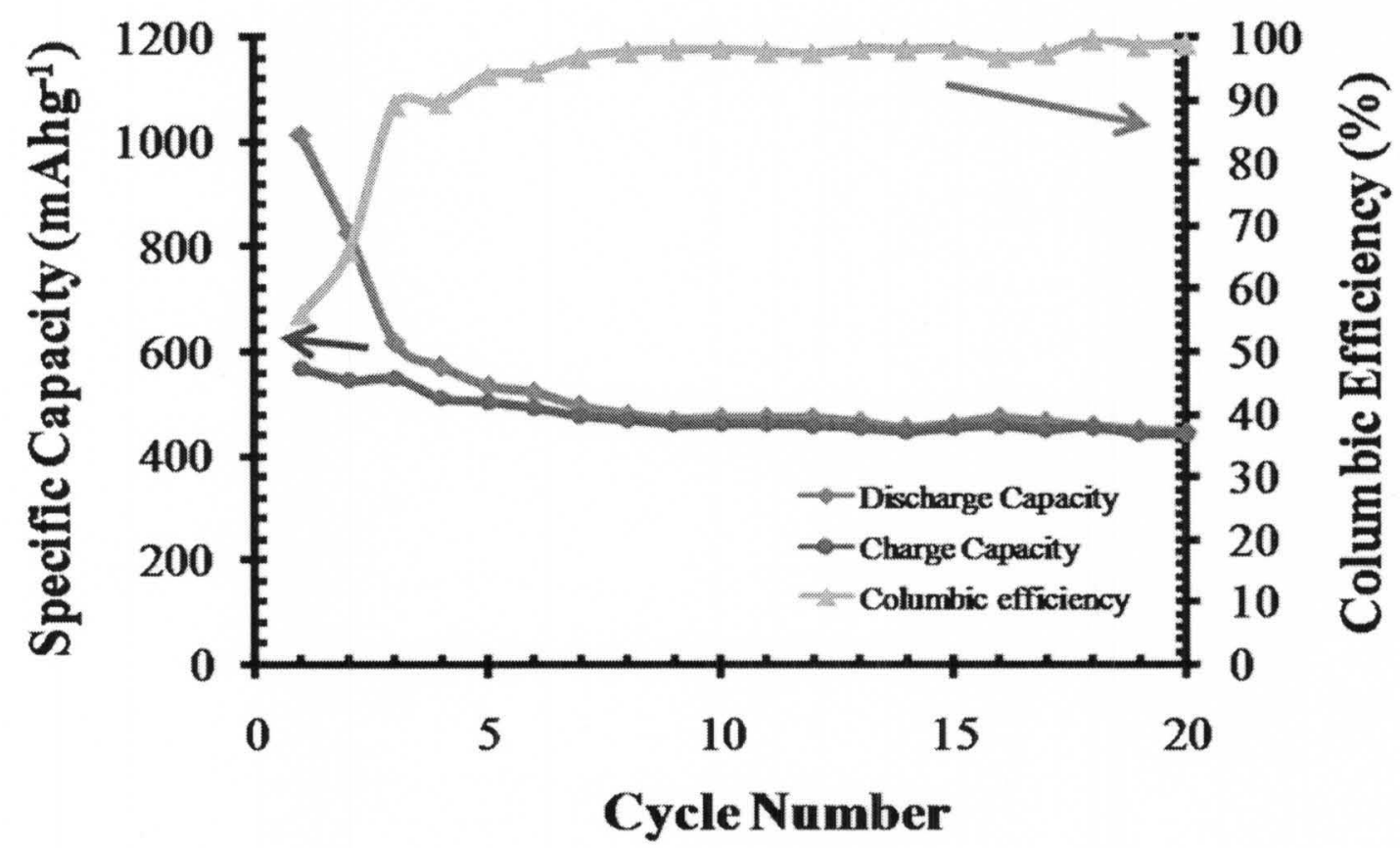

Figure 6.9: Cyclic performance at a current density of $25 \mathrm{mAhg}^{-1}$. Columbic efficiency is presented on the secondary axis to the right.

The initial discharge capacity is close to $1014 \mathrm{mAhg}^{-1}$ whereas the second discharge capacity is close to $826 \mathrm{mAhg}^{-1}$ at a current density of $25 \mathrm{mAg}^{-1}$. The initial high capacity indicates a $\mathrm{Li}$ stoichiometry of $\mathrm{Li}_{2.7} \mathrm{C}_{6}$ which is similar to that observed for the ball milled SWCNTs ${ }^{43}$. The second cycle ratio drops to $\mathrm{Li}_{2.3} \mathrm{C}_{6}$. It is however, interesting to see that there is also a steep drop in the third discharge cycle and then, the specific capacity remains fairly constant with a value of $443 \mathrm{mAhg}^{-1}$ after 20 cycles. The 
capacity in the third cycle corresponds to $\mathrm{Li}_{1.6} \mathrm{C}_{6}$ and remains fairly constant over the next 20 cycles corresponding to 1.2 lithium atoms per 6 carbon atoms $\left(\mathrm{Li}_{1.2} \mathrm{C}_{6}\right)$.

The specific capacity testing has been repeated with different mass loading of the samples and the results obtained correspond well with the capacity above. A specific capacity with a different mass loading has also yielded similar results. The capacity retention for the MWCNTs degrades to $\sim 250 \mathrm{mAhg}^{-1}$ after the first 6 cycles at a similar current density of $25 \mathrm{mAg}^{-1}$ as indicated in Section 6.2.1. It can be clearly seen that the CMTs have a specific capacity which is at least 1.5 times that of the MWCNTs. The charge-discharge curves for the CMTs are also presented in Figure 6.10 which shows a steady charge and discharge capacity with cycling.

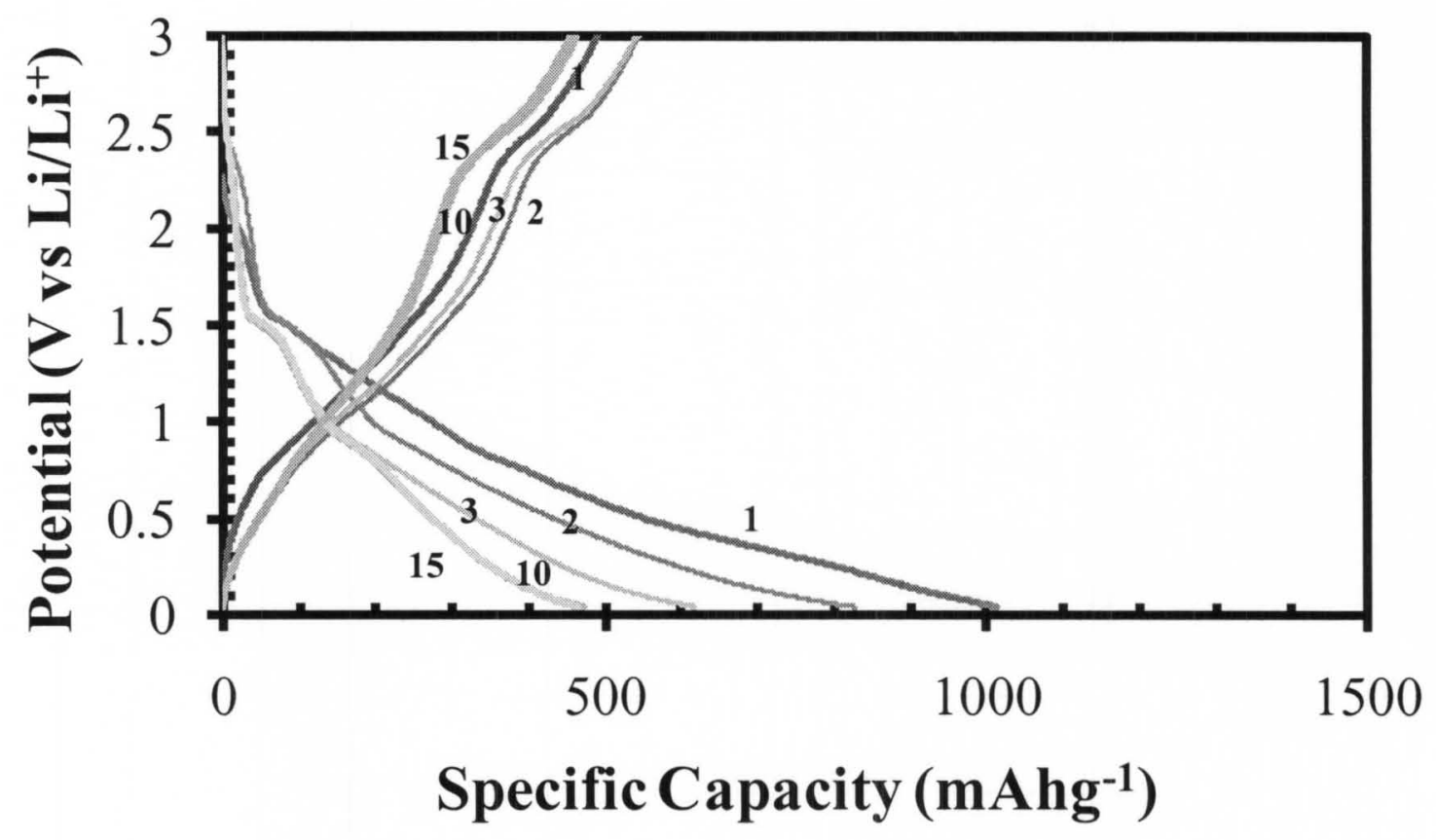

Figure 6.10: Electrochemical characteristics of CMTs with charge-discharge curves indicating minimal capacity loss after 3 cycles. 
Another interesting aspect of the present study is that the assembled cells were cycled between $3 \mathrm{~V}$ and $40 \mathrm{mV}, 40 \mathrm{mV}$ being higher than the usual low discharge voltages used for carbon based materials. Even though going to lower voltages of around $10 \mathrm{mV}$ can lead to significantly higher capacities, a higher potential will effectively prevent the $\mathrm{Li}$ from electroplating onto the material, which is a significant practical problem at high charge and discharge rates. The first cycle ratio $\mathrm{C}_{\text {rev }} /\left(\mathrm{C}_{\text {rev }}+\mathrm{C}_{\text {irrev }}\right)$ is close to $80 \%$, which is higher than for most of the carbonaceous materials ${ }^{32,71,156}$. Similarly, the second cycle ratio is $75 \%$, whereas the $\mathrm{C}_{\text {rev }} /\left(\mathrm{C}_{\mathrm{rev}}+\mathrm{C}_{\text {irrev }}\right)$ ratios from the third cycle is over $93 \%$ and reaches $98 \%$ in the subsequent cycles indicating a low irreversible capacity loss with cycling.

The columbic efficiency plotted on the secondary axis of Figure 3a shows 56\% and $66 \%$ efficiencies for the first and the second cycle respectively. The third cycle efficiency is $89 \%$ and thereafter, remains above $97 \%$ up to 20 cycles. The average capacity loss "c"over a period of " $n$ " number of cycles can be calculated as ${ }^{32,71}$

$$
C_{n} \equiv C_{1} /(1+c n)
$$

where $C_{n}$ and $C_{1}$ are the specific capacities in the $n^{\text {th }}$ and $1^{\text {st }}$ cycles respectively. The average capacity loss in 20 cycles for the CMTs during the charge process is $1.3 \%$ which is one of the lowest in carbon based electrodes ${ }^{71,156}$. Similarly, the average capacity loss during the discharge process is $6.4 \%$, in which the maximum capacity loss occurs during the first two cycles and then is quite low. Hence, both the charge and discharge capacity losses are quite low indicating the superior performance of CMTs. The aim of the next section is to understand the lithiation and de-lithiation characteristics of CMTs based on differential capacity curves and cyclic voltammetry. 


\subsubsection{Lithiation characteristics}

The specific capacity curves shown in Figure 6.10 indicate a two stage process of Li-ion intercalation and de-intercalation into the CMTs starting at a discharge voltage of $1.5 \mathrm{~V}$. The first stage is during low potentials of graphitized carbon which is at potentials below $0.3 \sim 0.4 \mathrm{~V}$. The second stage is the Li-ion intercalation at potentials higher than 0.4 $\mathrm{V}$ all the way up to $1.5 \mathrm{~V}$ as evident in the specific capacity curves in Figure 6.10 and from the differential capacity curves shown in Figure 6.11.

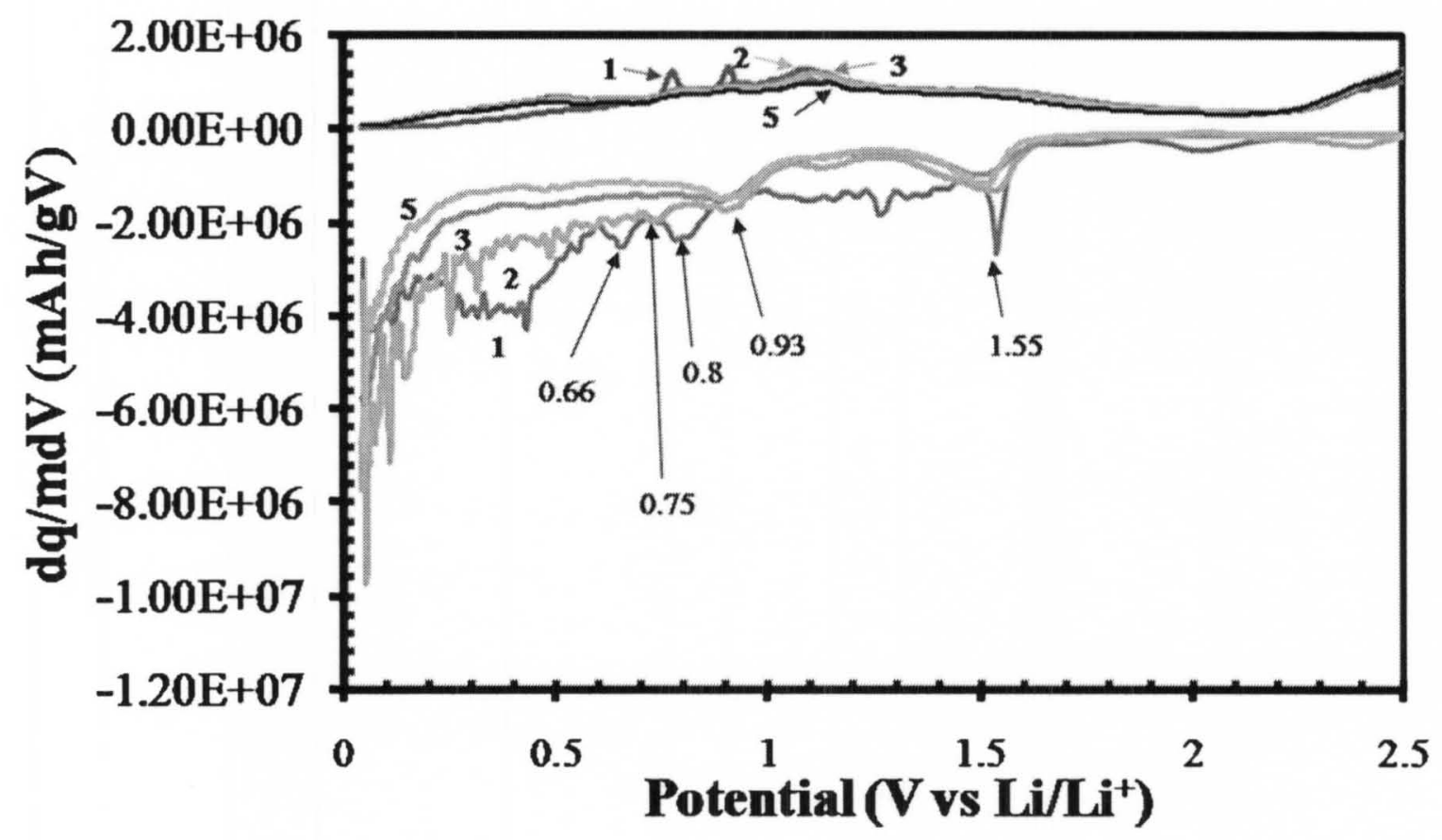

Figure 6.11: Differential capacity curves of the first 5 cycles of the CMTs cycled at a rate of $25 \mathrm{mAhg}^{-1}$ between $3 \mathrm{~V}$ and $40 \mathrm{mV}$.

The charge capacity in the range of 2.3 to $3 \mathrm{~V}$ is close to $100 \mathrm{mAhg}^{-1}$. Good capacity retention in this region can be attributed to pseudocapacitance behavior which occurs due to the high attraction between the Li-ions and the other charged species during 
$\mathrm{Li}$ intercalation as a result of which the removal of the $\mathrm{Li}$ ions and the electrical double layer (Helmholtz layer) becomes less favorable. The second stage intercalation happens at the disordered graphite array stackings which lead to turbostratic disorders as evident from the Raman spectroscopy and TEM analysis. The first discharge cycle differential capacity curve shows peaks at $0.66 \mathrm{~V}$ and $0.8 \mathrm{~V}$ which correspond to the decomposition of the electrolyte and its reaction with the anode surface forming the solid electrolyte interface (SEI). Evidently, there is a small peak during the $2^{\text {nd }}$ discharge cycle at $0.75 \mathrm{~V}$ which indicates the further formation of the SEI layer. Further differential capacity curves until the fifth cycle do not indicate the presence of the peaks at $0.66 \mathrm{~V}$ and $0.8 \mathrm{~V}$ confirming the fact that the SEI is formed only during the first two cycles which corresponds well with a significant drop in capacity during the first 2 discharge cycles followed by low capacity loss over the next 20 cycles.

The first DC curve shows a redox peak at $1.55 \mathrm{~V}$ and the second cycle shows two distinct peaks at $0.93 \mathrm{~V}$ and $1.54 \mathrm{~V}$. These peaks arise due to the Li-ion diffusion and intercalation into the open ends and/or from the defect sites on the walls of the CMTs ${ }^{157-}$ 159. Moreover, these peaks also indicate shorter Li-ion diffusion lengths and lower diffusion barriers inside the material. The Li-ion diffusion time constant into a material can be deduced as $t=L^{2} / D$ where " $L$ " is the Li-ion diffusion length inside the material and " $\mathrm{D}$ " is the Li-ion diffusion coefficient inside the material. " $\mathrm{D}$ ", inside the carbonaceous materials decreases with either the decrease in the diffusion length or an increase in the diffusion coefficient. As the time constant decreases, the power capability of the material increases. Due to the nanodomains of graphite in the walls of the CMTs, Li-ion diffusion lengths decrease considerably thereby increasing the rate capability. 
Cyclic voltammetry curves presented in Figure 6.12 clearly indicate peaks at 1.3 $\mathrm{V}, 0.62 \mathrm{~V}$ and $0.25 \mathrm{~V}$. The peak $1.3 \mathrm{~V}$ corresponds to decomposition of linear carbonate solvent which in this case is DEC. Similarly, the peak at $0.62 \mathrm{~V}$ corresponds to the decomposition of $\mathrm{EC}$ which is observed for about 5 cycles corresponding well with the drop in the capacity for the first 5 cycles. The peak at $0.25 \mathrm{~V}$ corresponds to the $\mathrm{Li}$-ion intercalation into CMTs which is also observed during the reverse process (deintercalation).

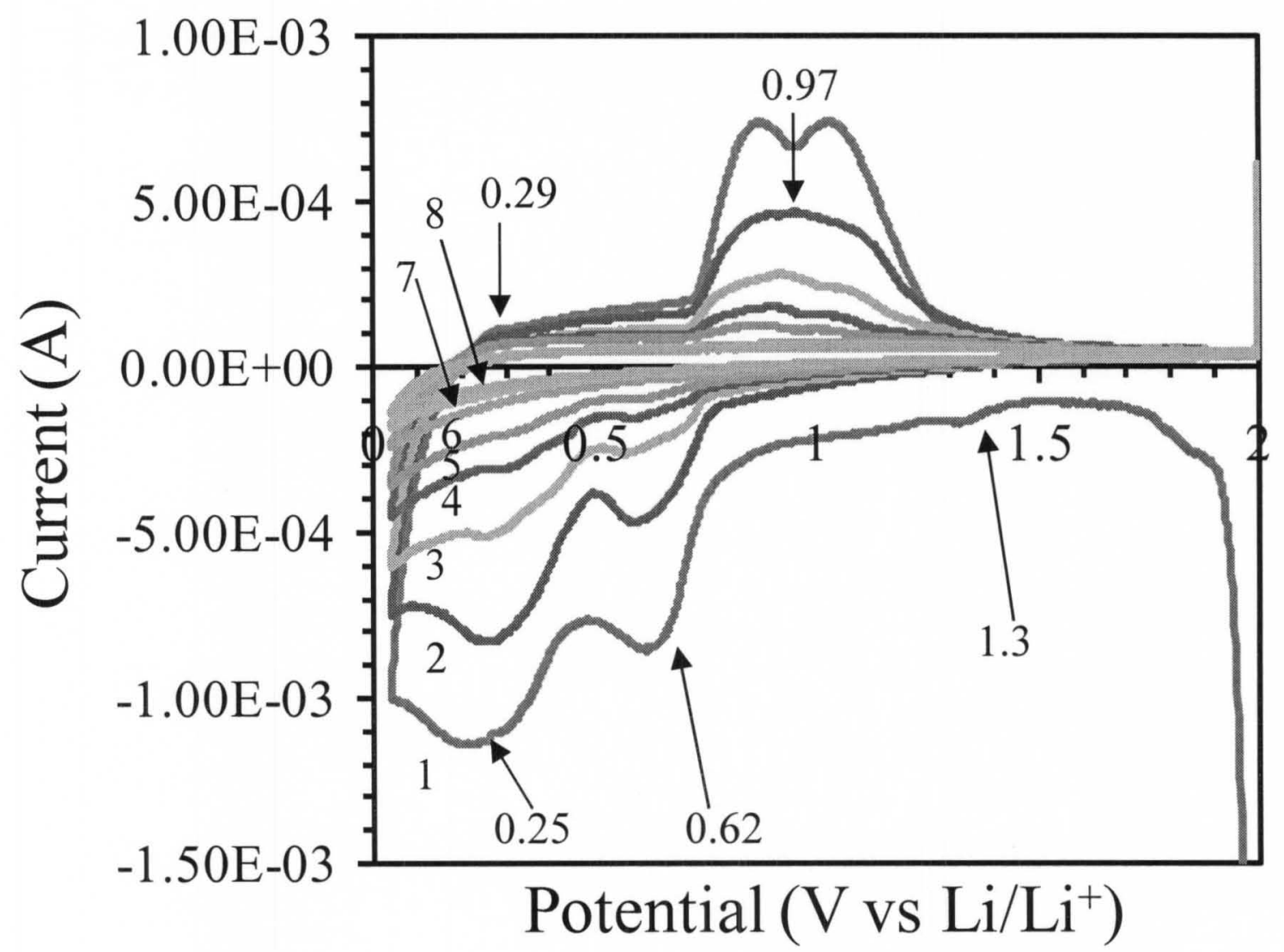

Figure 6.12: Cyclic voltammetry of CMTs indicating different peaks during the chargedischarge cycles for the first 8 cycles. 


\subsubsection{High rate performance}

The specific capacity retention of these materials at different current densities is shown in Figure 6.13. It can be seen that the CMTs retain a specific capacity of 135 $\mathrm{mAhg}^{-1}$ at a current density of $1.5 \mathrm{Ag}^{-1}$ (5C rate), and the good reversibility with changing rates indicate that CMTs have a robust structure and show tremendous promise on improving the rate capabilities of carbon based materials ${ }^{160}$. The data presented in Table I indicates that the synthesis of CMTs in the present work is a lot faster than the other carbonaceous materials with comparable rate performance indicating a tremendous potential for large scale synthesis. CMTs also exhibit good capacity retention for both low and high rates, unlike others presented in Table I.

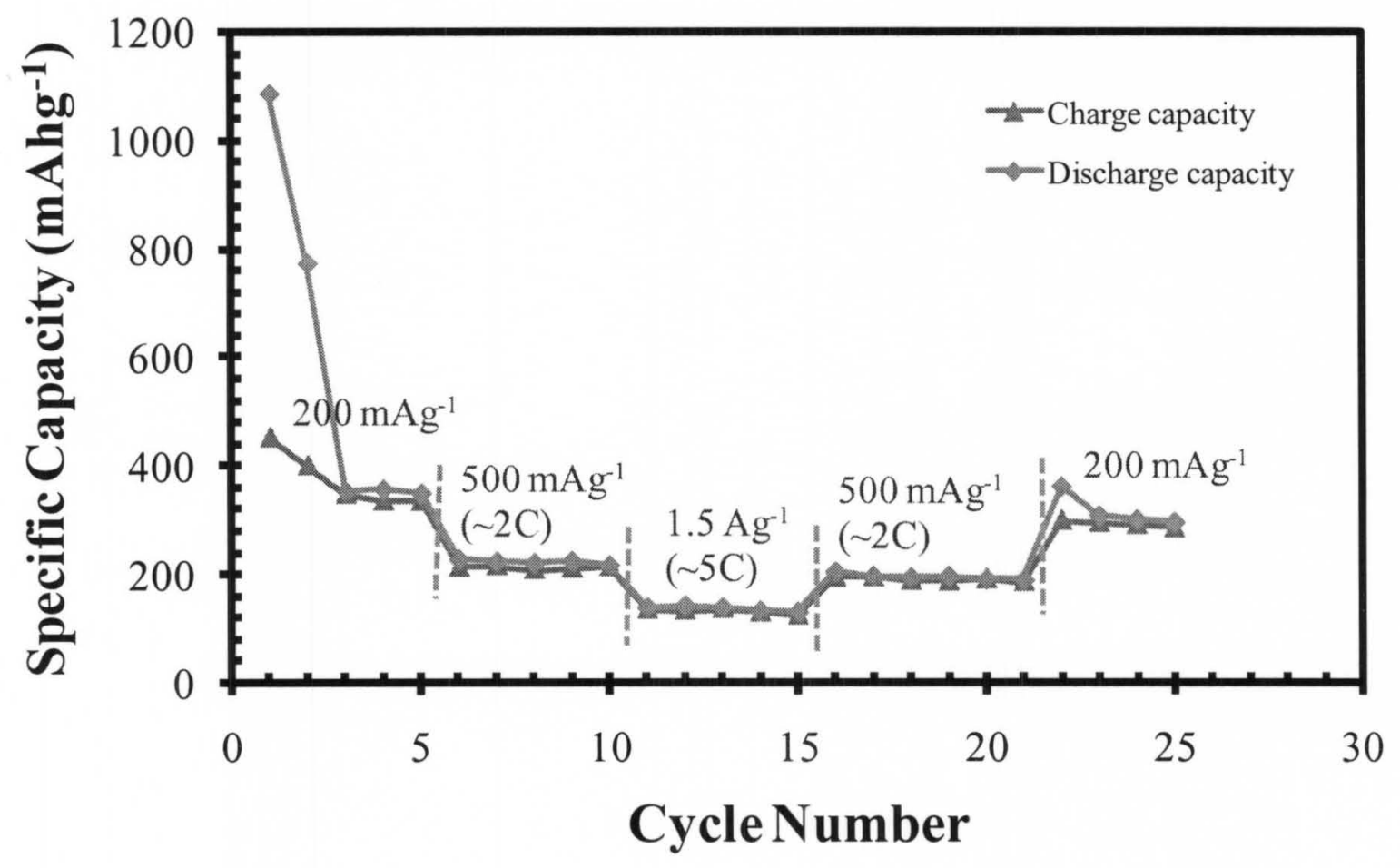

Figure 6.13. CMTs performance at different charge and discharge rates. 
Table 6.1. Rate performance of carbon microtubes compared with other carbonaceous materials.

\begin{tabular}{|c|c|c|c|c|c|c|}
\hline & $\begin{array}{l}\text { Rate } 1 \\
{\left[\mathbf{m A g}^{-1}\right]}\end{array}$ & $\begin{array}{l}\text { Specific } \\
\text { Capacity } \\
{\left[\mathrm{mAhg}^{-1}\right]}\end{array}$ & $\begin{array}{l}\text { Rate } 2 \\
{\left[\mathbf{A g}^{-1}\right]}\end{array}$ & $\begin{array}{l}\text { Specific } \\
\text { Capacity } \\
{\left[\mathrm{mAhg}^{-1}\right]}\end{array}$ & $\begin{array}{l}\text { Large area } \\
\text { synthesis } \\
\text { feasibility }\end{array}$ & Reference \\
\hline $\begin{array}{l}\text { Carbon } \\
\text { microtubes }\end{array}$ & 25 & $\begin{array}{ll}443 & (20 \\
\text { cycles }) & \end{array}$ & 1.5 & $\begin{array}{l}135 \\
\text { cycles })\end{array}$ & $\begin{array}{l}150 \mu \mathrm{m} \text { film } \\
\text { (10 minutes) }\end{array}$ & 160 \\
\hline $\begin{array}{l}\text { Carbon } \\
\text { nanofibers }\end{array}$ & $\sim 37$ & $\begin{array}{l}461 \\
\text { cycles })\end{array}$ & 3.0 & $\begin{array}{l}\sim 170(10 \\
\text { cycles })\end{array}$ & $>1$ hour. & 56 \\
\hline $\begin{array}{l}\text { Porous } \\
\text { carbon } \\
\text { monoliths }\end{array}$ & $\sim 75$ & $\begin{array}{l}<500 \quad(40 \\
\text { cycles })\end{array}$ & 22.0 & $\begin{array}{l}\sim 70(>10 \\
\text { cycles })\end{array}$ & $\begin{array}{l}2 \mathrm{~mm} \text { film } \\
\text { (>6days) }\end{array}$ & 161 \\
\hline $\begin{array}{l}\text { Carbon } \\
\text { nanosprings }\end{array}$ & 50 & $\begin{array}{l}\sim 420 \\
\text { cycles })\end{array}$ & 3.0 & $\begin{array}{l}160(>10 \\
\text { cycles })\end{array}$ & $>12$ hours. & 61 \\
\hline MWCNTs & - & - & 3.0 & 47 & $\begin{array}{l}\text { Commerciall } \\
\text { y available }\end{array}$ & 61 \\
\hline $\begin{array}{l}\text { Ball milled } \\
\text { CNTs }\end{array}$ & 50 & $\begin{array}{l}\sim 595 \text { after } \\
1 \text { cycle }\end{array}$ & - & - & $>10$ hours. & 162 \\
\hline
\end{tabular}

\subsubsection{Lithiation mechanism in CMTs}

\subsubsection{Post-lithiation analysis}

The post-lithiated CMT samples have been characterized to better understand characteristics of lithium intercalation and de-intercalation. The Raman spectra for both the pure and the post-lithiated CMTs are presented in Figure 6.14. It can be seen that the $\mathrm{G}$ band shifts downwards by $25 \mathrm{~cm}^{-1}$ after lithiation. The intercalation of the positive Liions (donor in this case) changes the electron density of the graphite layers in which, the charge is transferred to $\pi$ bonds of graphite resulting in the weakening of C-C intralayer 
bonds, leading to a downward shift of the G band in-plane stretching ${ }^{152}$. Similarly, the broadening of the $\mathrm{D}$ band as well as the blue shift by $20 \mathrm{~cm}^{-1}$ indicates that the graphene layers in the CMTs are further ruptured ${ }^{154}$. The additional peaks between $1100-1400 \mathrm{~cm}^{-1}$ indicate that the energy states at these frequencies become active at finite material sizes, attributed to the random lithium doping inside the material.

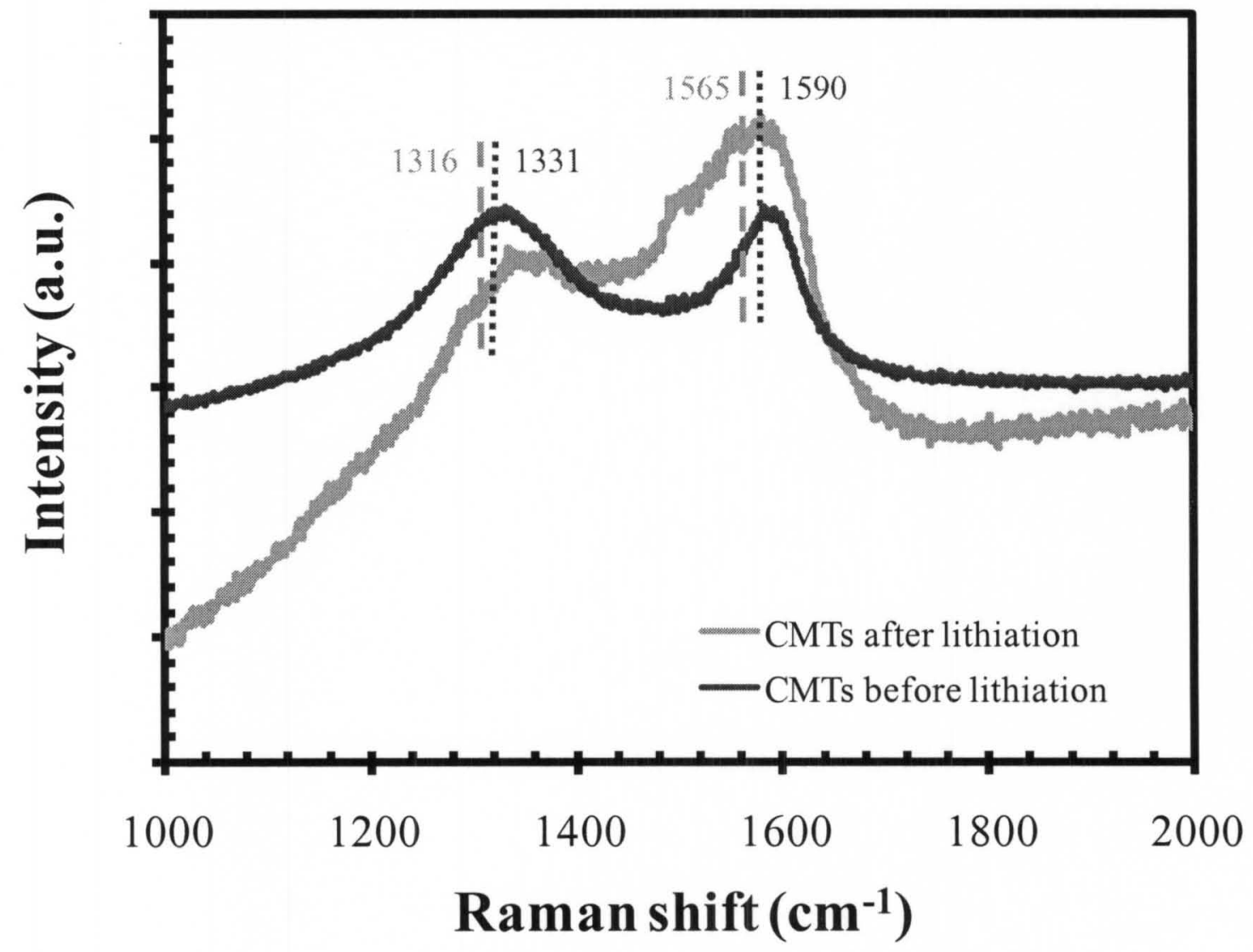

Figure 6.14: Post-lithiation analysis using Raman spectroscopy after 20 charge-discharge cycles for CMTs.

The XRD analyses in Figure 6.15 indicates the disappearance of the other phases of graphite seen in the pure XRD spectra followed by the widening of the (002) peak of 
graphite. Such peak broadening is typical of intercalated carbon compounds ${ }^{163}$. Additionally, a small peak arises pertaining to the graphite (004) orientation.

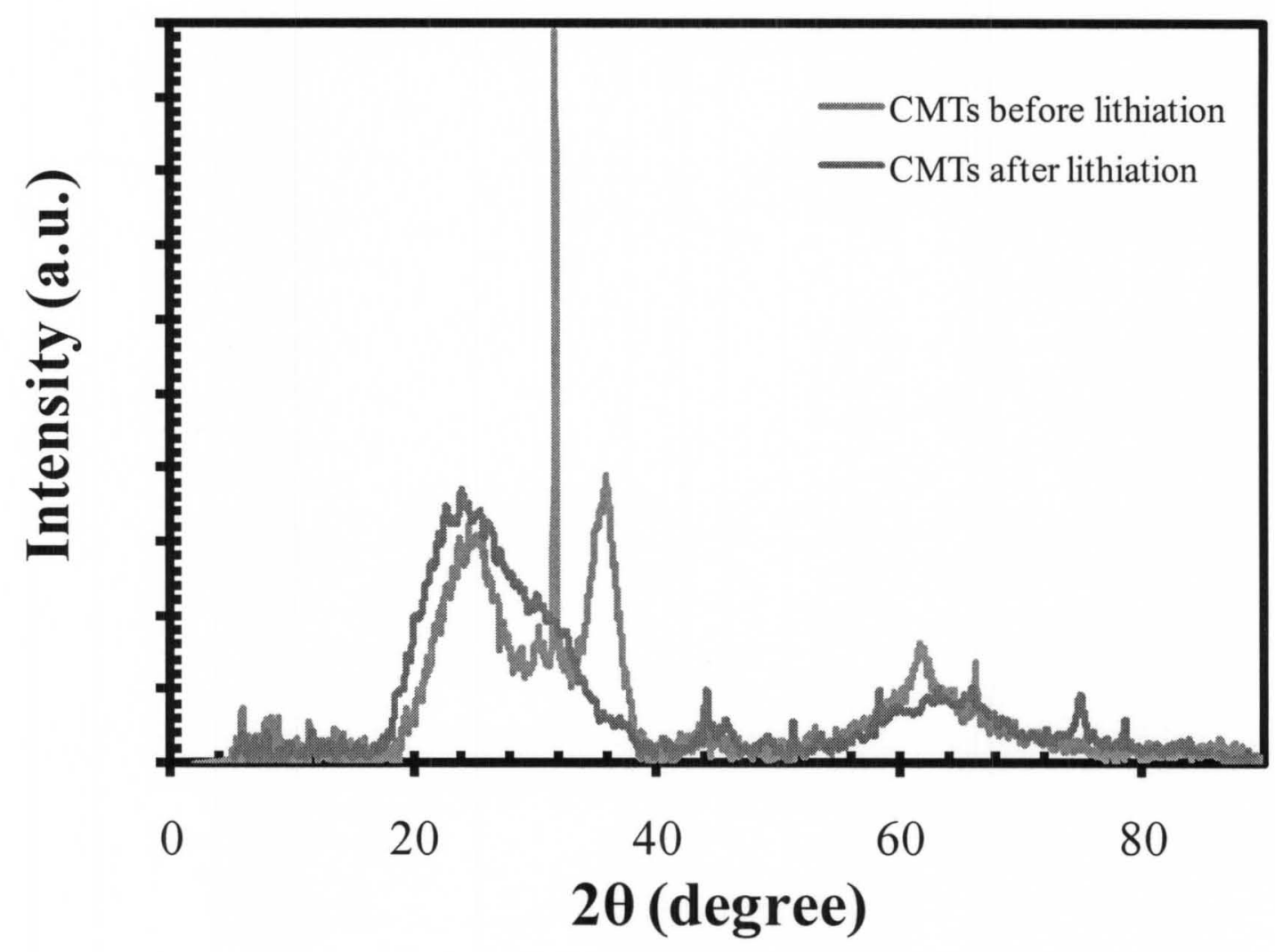

Figure 6.15. Phase and structural changes in the material characterized by XRD after 20 cycles.

The stacking length of the CMTs $\mathrm{L}_{\mathrm{c}}$ can be calculated using the (002) peak of the XRD spectra from the Scherrer formula ${ }^{164}$

$$
L_{c} \equiv \frac{0.89 \lambda}{B_{c} \cos \theta}
$$

where $\lambda$ is the $x$-ray wavelength and $\theta$ is the (002) Bragg peak. The crystallite size $L_{a}$ of the graphite can be calculated using the intensity ratios of D and G bands from the Raman spectrum. It has been proven that the following Knight's relationship holds for graphite 


$$
L_{a} \equiv C\left(\frac{I_{D}}{I_{G}}\right)^{-1}
$$

where $\mathrm{C}=8.3$ is a constant obtained from the Raman spectroscopy laser wavelength $(632.8 \mathrm{~nm})^{165}$ and $\mathrm{L}_{\mathrm{a}}$ has the units of $\mathrm{nm}$. The intensity ratios in this case are the areas under the peak unlike some of the other studies which compare the ratios of peak amplitude. In the case of pure CMTs, the stacking length is $1.92 \mathrm{~nm}$ with a lattice spacing of $3.53 \AA$ which accounts for about 5 to 6 stacking layers of graphite with a crystallite size $\left(\mathrm{L}_{\mathrm{a}}\right)$ of $7.9 \mathrm{~nm}$. In the case of lithiated CMTs, it can be seen that the stacking length has a significant drop to $0.76 \mathrm{~nm}$ with 2 layers of graphite while the crystallite size increased to $23.7 \mathrm{~nm}$ which can be explained by the fact that the CMT walls become more disorderly with Li-ion intercalation and de-intercalation.

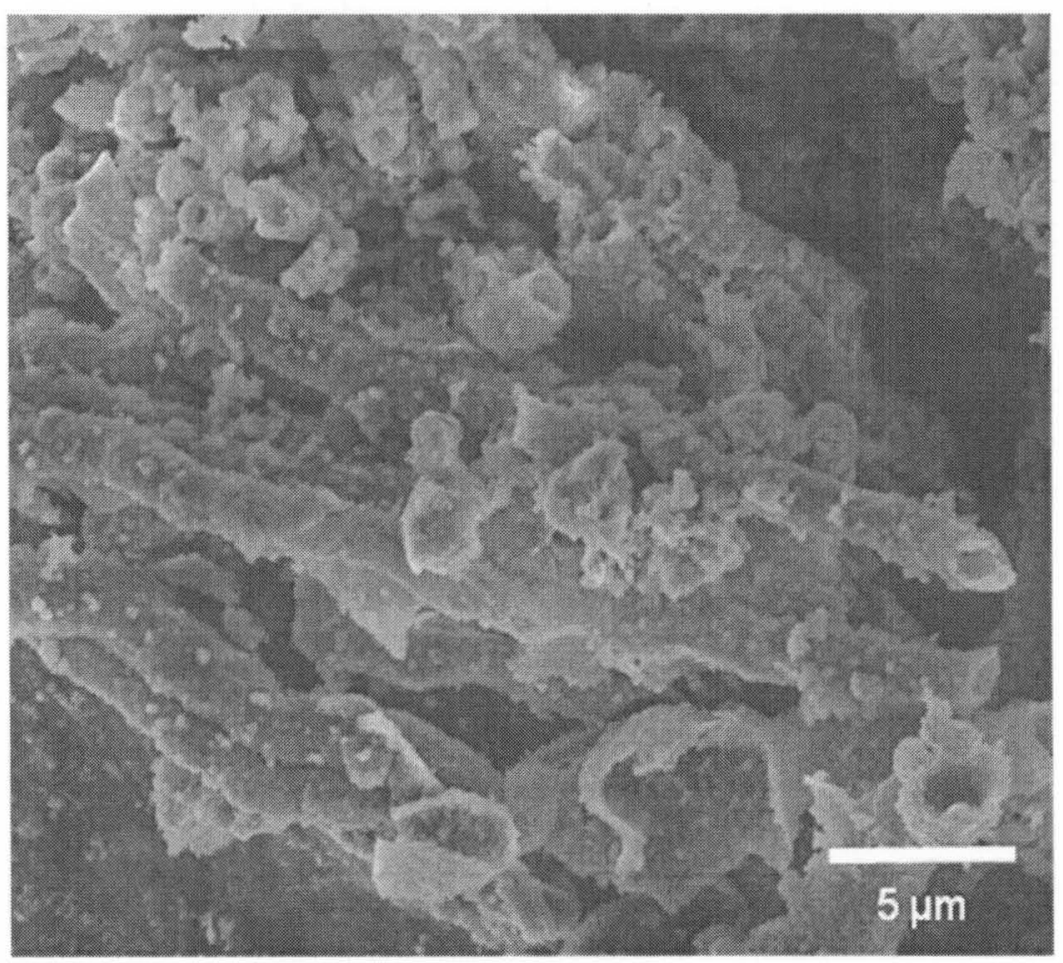

Figure 6.16: SEM image showing the physical structure of the CMTs. 
The SEM image in Figure 6.16 of the post-lithiated sample indicates the stable physical tube structure of the CMTs even after 20 cycles but gives no information about the graphite nanodomain structures. Figure 6.17 shows the TEM images of CMTs before and after lithiation. The high resolution images show the presence of graphite nanodomains even after lithiation. The low-resolution image shows the presence of SEI layer on the surfaces of thin walls of CMTs but is not predominant. Nano-graphite domains of $\sim 8 \mathrm{~nm}$ exist within the thin walls of CMTs which are close to $\sim 50 \mathrm{~nm}$. Post-lithiated images show graphite domains with an increased domain size consistent with the results obtained using the Raman and XRD analysis, but still retain the crystallinity.

In addition, the low resolution image shows the presence of SEI layer but is not predominant. It is also clearly visible in these images that SEI is not severe on the nanodomains probably because the graphite planes are not open ended in this case but are randomly oriented and embedded in the wall of the CMT among amorphous matrix. Moreover, the differential capacity curves indicate the formation of the SEI layer during the first 2 cycles which corresponds well with a significant loss of capacity for the first two cycles. Once the lithium ions pass through the SEI layer on the surface, they intercalate with carbon in the amount of $1.2 \mathrm{Li}$ ions per 6 carbon atoms which leads to high capacity retention.

Post-lithiated SEM and TEM images show a stable structure for CMTs which shows that these materials can serve as ideal base materials to create new hybrid nanoarchitectures which can lead to significant advancements in developing new viable electrode materials in LIBs. 

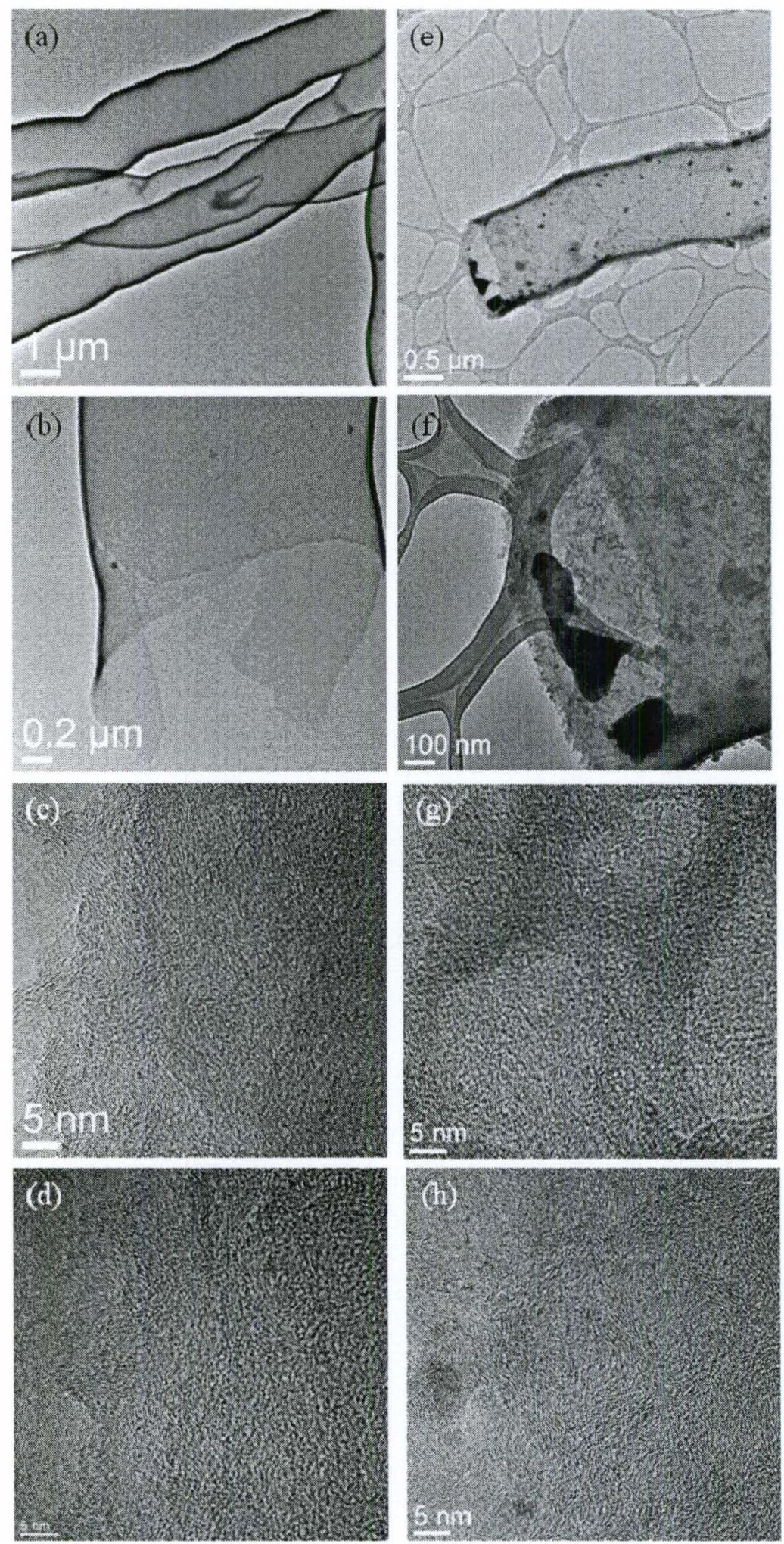

Figure 6.17: TEM images of CMTs (a, b, c, d) Pure CMTs devoid of gallium after acid treatment prior to cycling measurements. $(\mathrm{e}, \mathrm{f}, \mathrm{g}, \mathrm{h}) \mathrm{CMTs}$ after 20 cycles of lithiation 
and de-lithiation showing the presence of SEI layer but the graphite nanodomains are still clearly evident on the surface which are the pathways for Li-ion diffusion.

\subsubsection{Proposed lithiation mechanism}

Based on the results obtained in the previous section, a simple mechanism can be proposed for the Li-ion intercalation into the CMTs. The mechanism of Li-intercalation and de-intercalation can be explained as follows:

- The CMT walls have nanographite domains of graphite which precisely have 5-6 graphene layers with a crystallite size of $7.9 \mathrm{~nm}$.

- The Li-ions enter both the inside as well as the outside walls through the defect sites along with the diffusion from the tube ends into these nanographite domains.

- Repeated Li-ion cycling cracks the defect sites further showing a significant drop in the stacking length of the nanodomains to about 2 graphene layers with an increase in the crystallite size, probably from the volume expansion of the crystallite on lithium intercalation.

- Overall, the disorderliness of the nanodomains increases as a result of the lithiation.

In principle, the Li-ions follow the model proposed by Dahn and coworkers ${ }^{66,166}$ which proposes that $\mathrm{Li}$-ions are adsorbed on both sides of the graphene sheets in disordered structures, in the defect structures comprised of stacking faults ${ }^{167}$ and in the internal interfaces which can explain the excessive storage of lithium, more than the theoretical capacity of $1 \mathrm{Li}$ ion per 6 carbon atoms. 


\subsection{Summary}

MWCNTs have shown low capacity retention of $\sim 250 \mathrm{mAhg}^{-1}$ after 6 cycles. For the first time, CMTs have been synthesized on large area substrates and tested as possible anode candidates for Li-ion batteries. CMTs have shown a capacity of $450 \mathrm{mAhg}^{-1}$ after 20 cycles, $\sim 1.5$ times higher than MWCNTs. The average charge capacity loss was reported to be $1.3 \%$, which is quite low for carbon based materials. CMTs have also shown to be able to withstand high rates with capacity retention of $135 \mathrm{mAhg}^{-1}$ at a current density of $1.5 \mathrm{Ag}^{-1}$ and then reversibly achieving the original capacity at lower rates. It is inferred from the post-lithiation analysis that the nanodomains of graphite in the walls of the CMTs and the open ends of CMTs provide various channels for Li intercalation. The Li-ion diffusion lengths can significantly decrease because of this resulting in excellent performance of the CMTs over a wide range of charge-discharge rates. 


\section{CHAPTER 7}

\section{NANOWIRE ARRAYS AS ANODES AND BASE MATERIALS FOR HYBRID ARCHITECTURES}

\subsection{Introduction}

Chapters 3 and 4 have focused on the synthesis and characterization of nanowires in the powder form as anodes. However, fabrication of anodes using these powders requires polymeric binder and conductive carbon leading to increased weight. However, nanowire arrays grown directly on conducting substrates eliminate additional materials as in the case of powders leading to low fabrication costs and higher specific energy material. Tin oxide based hybrid material is shown to retain high capacity and good rate capability but transition metal oxides like molybdenum oxide $\left(\mathrm{MoO}_{3}\right)$ and tungsten oxide $\left(\mathrm{WO}_{3}\right)$ undergo lower volume expansions with better stability, and form the basis for this chapter.

Carbon based materials do not sustain extreme temperatures, undergo lithium plating at low intercalation potentials and have lower battery voltages which led to study of these metal oxides as potential candidates to overcome these obstacles. However, bulk $\mathrm{MoO}_{3}$ material has shown high initial capacity but significant capacity fading with cycling. $\mathrm{MoO}_{3}$ doped with sodium and tin have also shown high initial capacities but suffer from capacity degradation as well. Recently, Dillon et al. ${ }^{105}$ have shown that $\mathrm{MoO}_{3}$ nanoparticles retain a capacity of $630 \mathrm{mAhg}^{-1}$ for up to 150 cycles when cycled between 
$3.5 \mathrm{~V}$ and $0.005 \mathrm{~V}$. Ball milled $\mathrm{MoO}_{3-\mathrm{x}}$ samples have also been shown to exhibit a high initial discharge capacity of $1100 \mathrm{mAhg}^{-1}$ but the material undergoes capacity degradation with a final capacity retention of $\sim 620 \mathrm{mAhg}^{-1}$ after 35 cycles $^{99}$. A significant drawback in the above mentioned studies is that the capacity is obtained over the entire potential range which limits its application as an anode in practical batteries. In addition, nanoparticles contain grain boundaries limiting the conductivity of the material.

The primary focus of the chapter is to study the performance of 1-dimensional $\mathrm{MoO}_{3-\mathrm{x}}$ and $\mathrm{WO}_{3-\mathrm{x}}$ nanowires arrays as anode materials for LIBs. Particularly, these arrays have been synthesized directly on conducting substrates providing direct electron conduction channels. 1-D nanowires also provide better electronic and ionic conduction pathways than their nanoparticle counterparts. To our knowledge, this is the first study on the use of both $\mathrm{MoO}_{3-\mathrm{x}}$ and $\mathrm{WO}_{3-\mathrm{x}}$ 1-D nanowires as anode materials. These studies are aimed to produce stable, high capacity materials, in a small potential window for their practical application as anode materials.

\subsection{Synthesis of nanowire arrays directly on conducting substrates}

\subsection{1 $\mathrm{WO}_{3-\mathrm{x}}$ nanowire arrays}

$\mathrm{WO}_{3-\mathrm{x}}$ nanowires are synthesized in a hot filament CVD reactor directly onto conducting substrates like stainless steel and platinum. Tungsten filament serves as the metal source which is heated to $2000 \mathrm{~K}$. The feed gases used in this case are air or oxygen and argon. The conditions pertain to lean oxygen conditions for the growth of nanowire arrays. The experiments are carried out for a period of 10 minutes. 


\subsection{2 $\mathrm{MoO}_{3-\mathrm{x}}$ nanowire arrays}

$\mathrm{MoO}_{3-\mathrm{x}}$ nanowires are synthesized in $10 \mathrm{sccm}$ of oxygen on metal substrates in a hot-filament chemical vapor deposition reactor. The deposition source is a molybdenum filament which is resistively heated to emit Mo vapor which reacts with the oxygen to deposit the oxide onto the surface. $\mathrm{MoO}_{3-\mathrm{x}}$ nanowires are grown as arrays on stainless steel and copper. The synthesis is carried out for duration of 30 minutes. Nanowire arrays are characterized for their morphology using SEM, XRD and Raman spectroscopy. The as synthesized nanowires on conducting substrates are used as electrodes for electrochemical measurements.

\subsection{Characterization of $\mathrm{WO}_{3-\mathrm{x}}$ arrays}

\subsubsection{Physical characteristics}

SEM images in Figure 7.1 indicate the array architecture of the $\mathrm{WO}_{3-\mathrm{x}}$ nanowires as seen from the top. Figure 7.1b specifically indicates the array architecture from the side. These nanowires are $<100 \mathrm{~nm}$ in diameter and up to 5 microns in length.
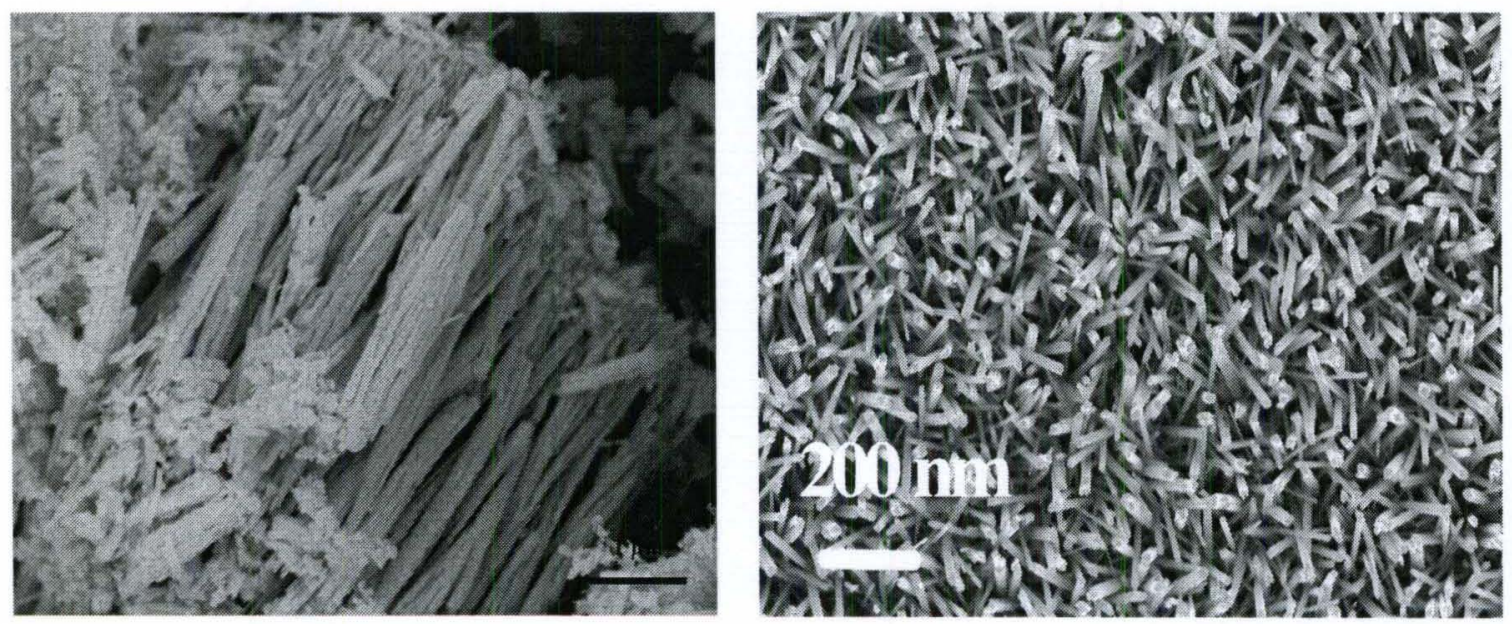

Figure 7.1: SEM images of as synthesized $\mathrm{W}_{18} \mathrm{O}_{49}$ nanowires. (a) Top view (b) Side view 
In some cases, nanowire bundles consisting of $<10$ nanowires are seen which is due to agglomeration caused by high temperature synthesis of the material. These nanowires directly grown on the substrates present interesting electronic conductivity enhancements. In addition, the density of the nanowires on the substrates is significantly high and is $\sim 10^{11} \mathrm{nwcm}^{-2}$. The nanowire diameters can be controlled by deposition conditions and oxygen/air flow. The as synthesized nanowires are deep blue in color. The $\mathrm{XRD}$ spectrum obtained for these nanowires indicates a monoclinic $\mathrm{W}_{18} \mathrm{O}_{49}$ phase which is oxygen deficient and is shown in Figure 7.2.

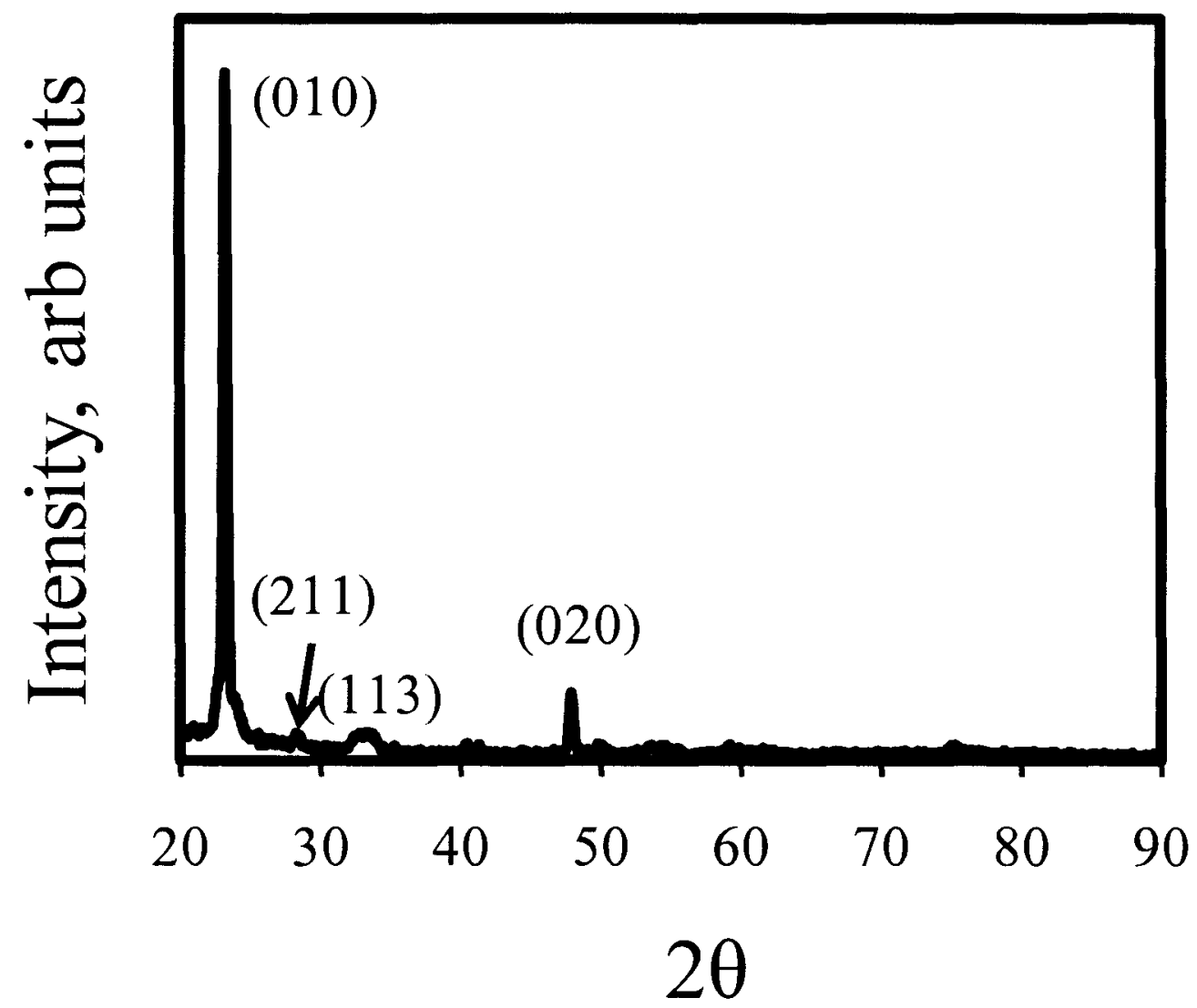

Figure 7.2: $\mathrm{XRD}$ spectrum of $\mathrm{W}_{18} \mathrm{O}_{49}$ nanowire array. 
Raman spectroscopy of these nanowires is presented in Figure 7.3. This spectrum shows four distinct peaks corresponding to $264,322,709$ and $803 \mathrm{~cm}^{-1}$ respectively. The lower wave number bands at 264 and $322 \mathrm{~cm}^{-1}$ correspond to the bending modes of the oxygen-tungsten bonds. Similarly, the higher wave number bands at 709 and $803 \mathrm{~cm}^{-1}$ correspond to the stretching modes of the W-O bonds. All these peaks correspond well with monoclinic $\mathrm{WO}_{3-\mathrm{x}}$ phase.

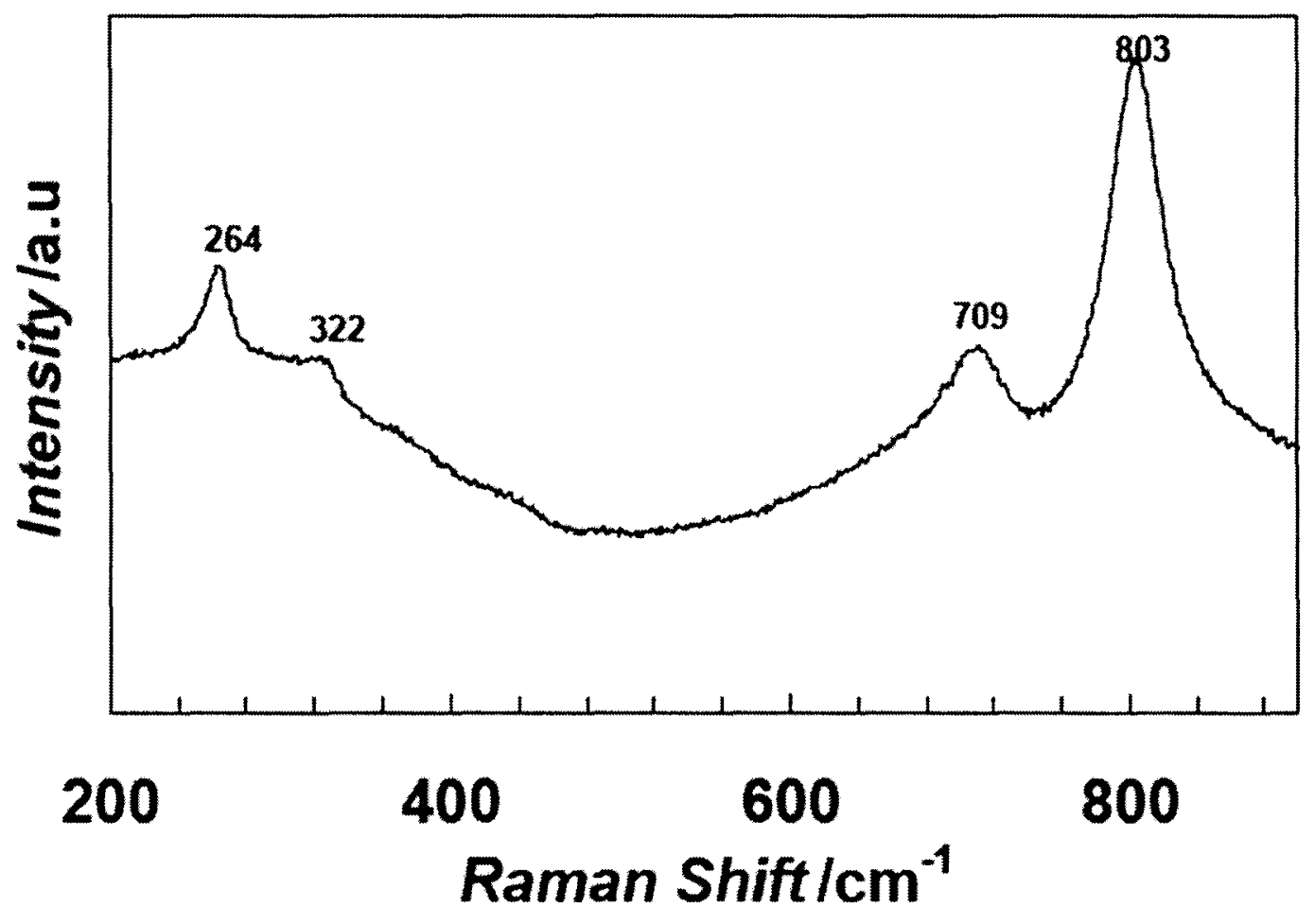

Figure 7.3: Raman spectrum of $\mathrm{W}_{18} \mathrm{O}_{49}$ nanowire array.

High resolution TEM image of the as synthesized $\mathrm{W}_{18} \mathrm{O}_{49}$ nanowires is shown in Figure 7.4. This image indicates a highly crystalline nanowire architecture with $<010>$ growth direction devoid of any amorphous oxide layer on the nanowire surface. 


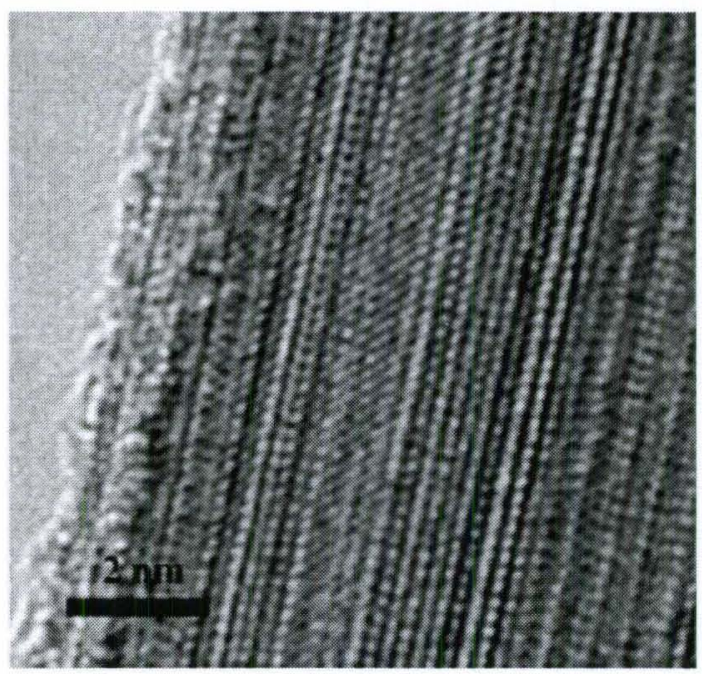

Figure 7.4: HRTEM image of an individual $\mathrm{W}_{18} \mathrm{O}_{49}$ nanowire showing high crystallinity all along the nanowire.

\subsubsection{Electrochemical characteristics}

The as synthesized $\mathrm{W}_{18} \mathrm{O}_{49}$ nanowires are characterized using electrochemical techniques to measure the specific capacity of the material. $\mathrm{W}_{18} \mathrm{O}_{49}$ nanowires were cycled between $40 \mathrm{mV}$ and $3.5 \mathrm{~V}$. These nanowires retain a specific capacity of $\sim 180$ $\mathrm{mAhg}^{-1}$ for up to 20 cycles which is presented in Figure 7.5. First discharge cycle capacity is $327 \mathrm{mAhg}^{-1}$, corresponding to more than $1.5 \mathrm{Li}$ ion per an atom of $\mathrm{W}_{18} \mathrm{O}_{49}$. All the measurements are performed at a current density of $25 \mathrm{mAg}^{-1}$. The columbic efficiency of the first cycle is $\sim 80 \%$ which is significantly high for metal oxide materials. The second and third cycle capacities are 220 and $178 \mathrm{mAhg}^{-1}$, respectively which remains constant until 20 cycles. The electrodes here have been cycled to a cutoff voltage of $40 \mathrm{mV}$ which prevents lithium plating. $\mathrm{Li}$ ions intercalate with $\mathrm{WO}_{3-\mathrm{x}}$ by the following mechanism:

$$
\mathrm{xLi}^{+}+\mathrm{xe}^{-}+\mathrm{WO}_{3} \leftarrow \rightarrow \mathrm{Li}_{\mathrm{x}} \mathrm{WO}_{3}(0 \leq \mathrm{x} \leq 1.5)
$$


$\mathrm{W}_{18} \mathrm{O}_{49}$ nanowires retain the theoretical capacity of $\sim 175 \mathrm{mAhg}^{-1}$ corresponding to $1.5 \mathrm{Li}$ for $\mathrm{W}$ atom. However, nanowire arrays show higher initial capacities because of additional defect sites on the material surface which promote higher amount of Li incorporation.

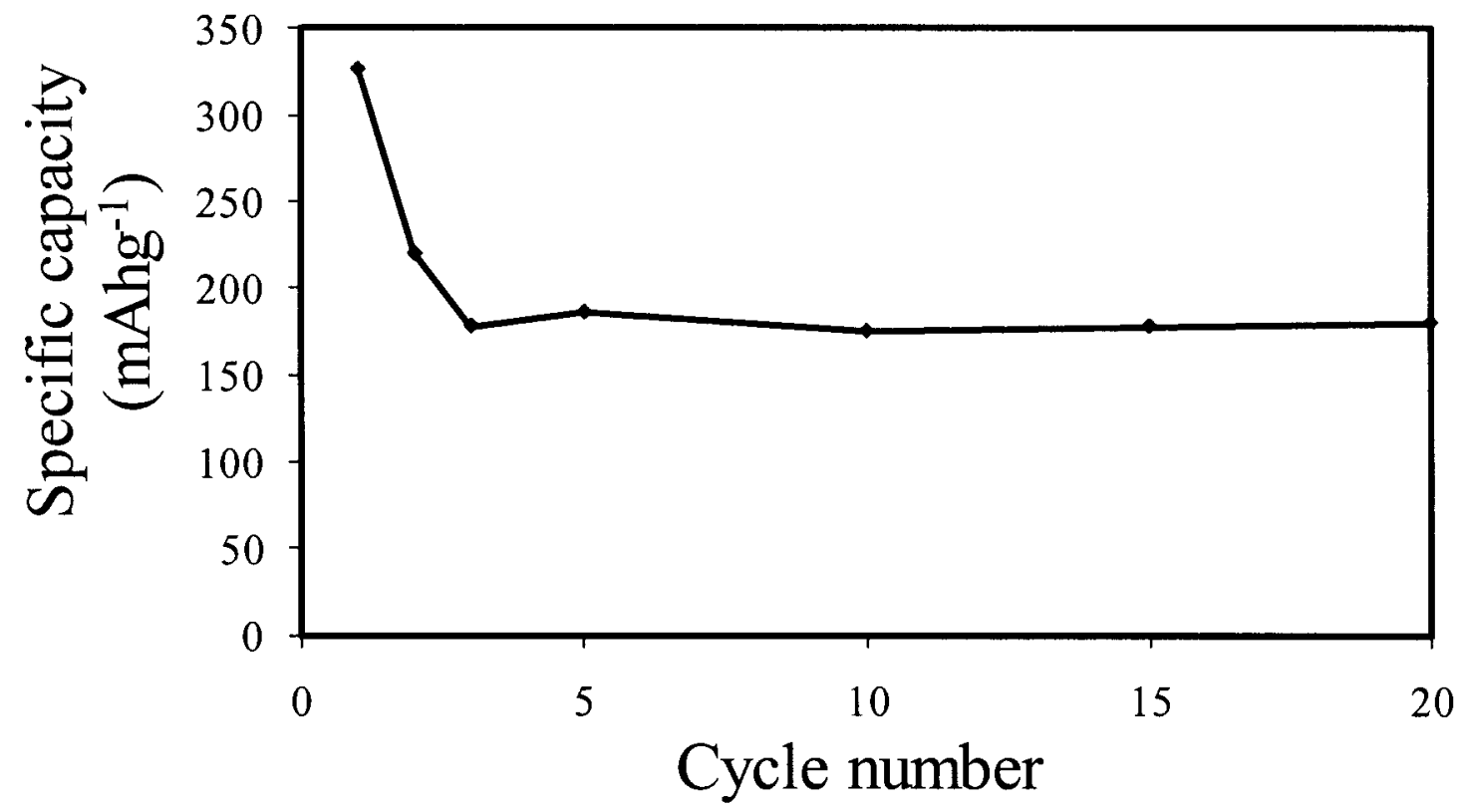

Figure 7.5: Specific capacity of $\mathrm{W}_{18} \mathrm{O}_{49}$ nanowires at a current density of $25 \mathrm{mAg}^{-1}$.

Figure 7.6 presents the first cycle charge and discharge of these nanowire arrays. It is clearly seen that the voltage has a constant plateau at $\sim 3 \mathrm{~V}$ during the first discharge cycle. Data pertaining to cycles $2-5$ is shown in Figure 7.7 . It is evident from this figure that most of the capacity is obtained in a voltage window of $0.7 \mathrm{~V}$ between $2.8-2.1 \mathrm{~V}$. Similarly, it can also be seen that the charge profile is constant in a small window of $\sim 0.1$ V. The loss in the capacity during the first two cycles is attributed to the electrolyte decomposition and the formation of SEI. It has been shown in chapter 6 that the high 
surface area of the nanowire materials can lead to the formation of SEI for the first few cycles leading to a loss in the capacity. Similar phenomenon is observed for these $\mathrm{W}_{18} \mathrm{O}_{49}$ nanowire arrays which undergo capacity loss during the first 3 cycles and can be attributed to SEI formation.

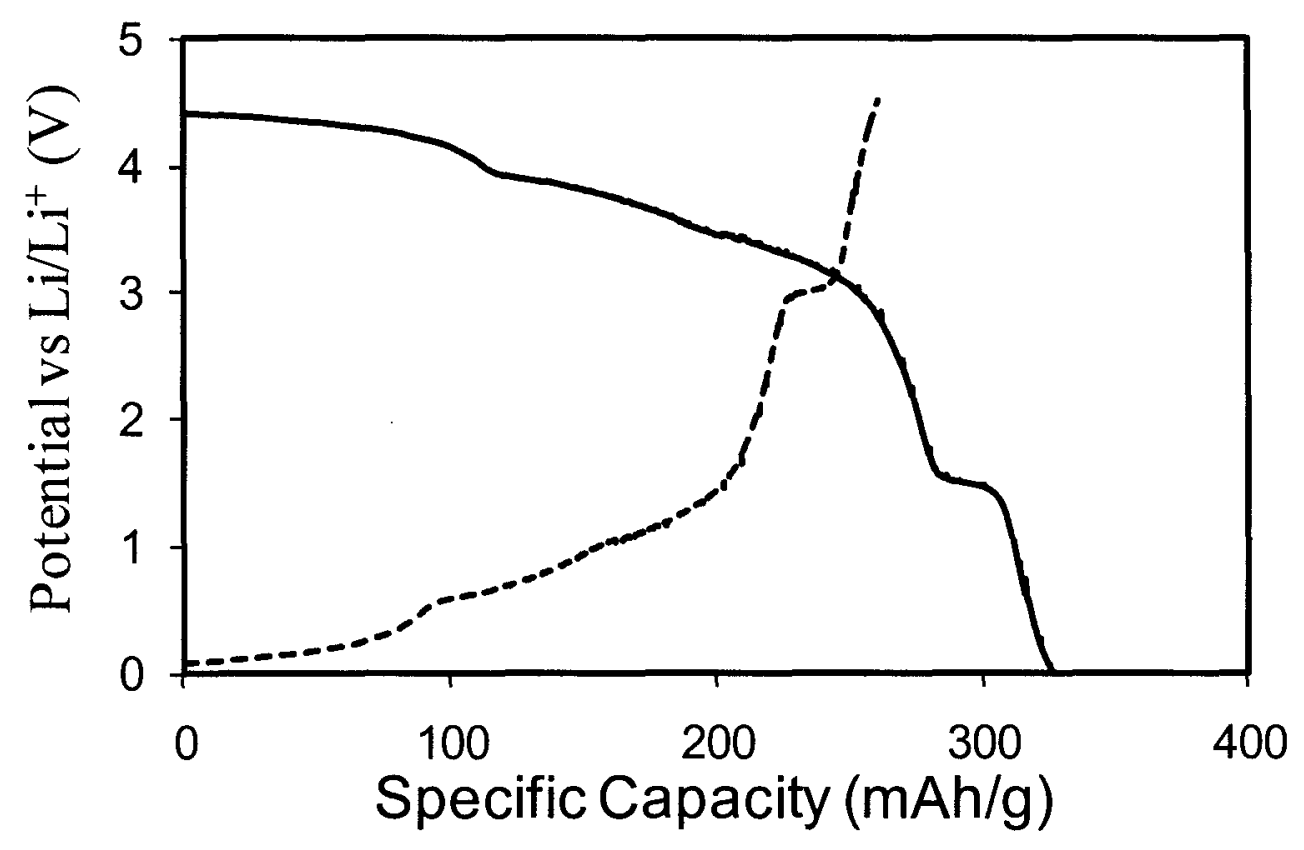

Figure 7.6: First cycle charge-discharge curves of $\mathrm{W}_{18} \mathrm{O}_{49}$ nanowire arrays.

However, it has to be realized that the voltage obtained in an actual battery $(\sim 2 \mathrm{~V})$ using these array anodes is lower than that obtained using the carbon based materials $(\sim 3.7-4 \mathrm{~V})$. But the energy density that can be obtained using these anodes is relatively high (2000 $\mathrm{mAhL}^{-1}$ ) which makes them particularly appealing for high power applications. Moreover, the high potential w.r.t $\mathrm{Li} / \mathrm{Li}^{+}$prevents the lithium plating occurring at low potentials (especially in the case of carbon based materials) paving the 
way for fabricating safer batteries. In addition, the synthesis of these materials using HFCVD is fairly inexpensive.

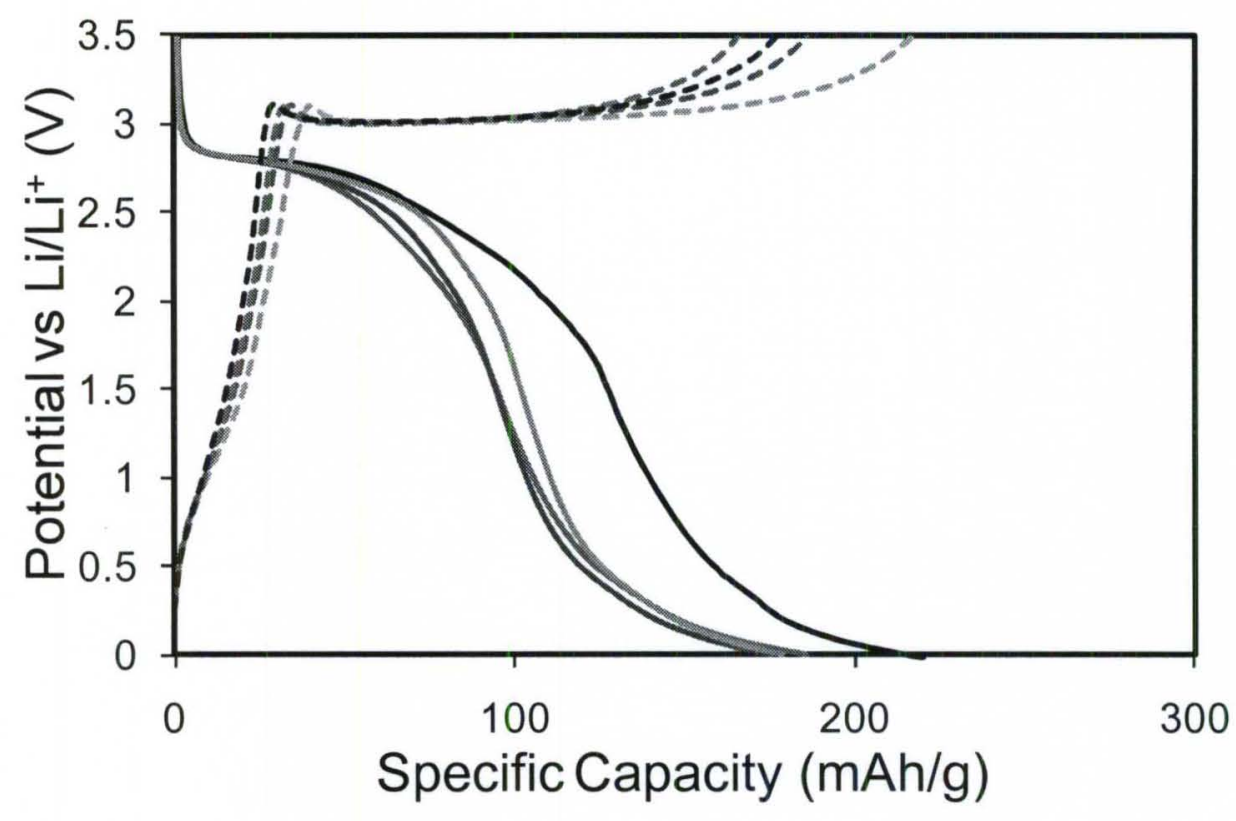

Figure 7.7: Charge-discharge cycles $2-5$ of the $\mathrm{W}_{18} \mathrm{O}_{49}$ nanowires.
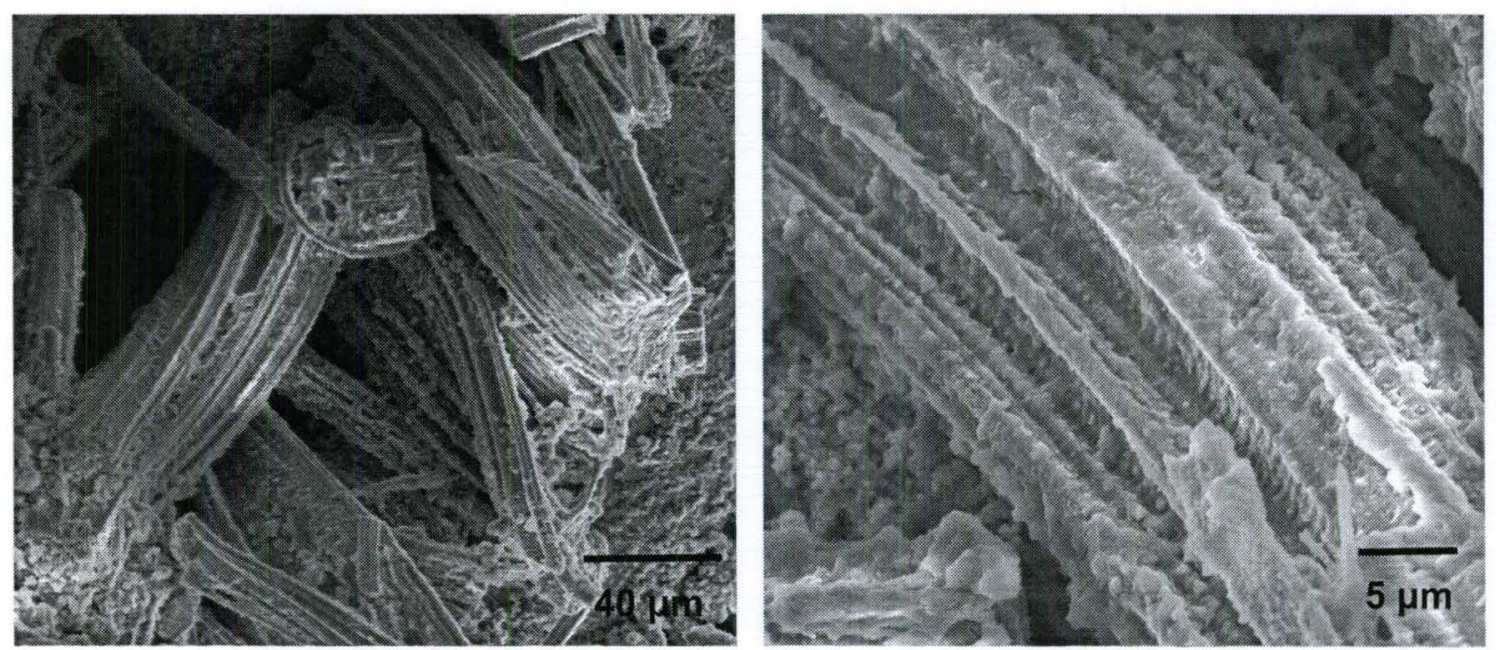

Figure 7.8: Post-lithiated SEM images of nanowire arrays after 20 charge-discharge cycles. 
SEM images in Figure 7.8 clearly show that the nanowire architecture is intact with minimal deformation which leads to constant capacity retention with cycling. Hence, $\mathrm{W}_{18} \mathrm{O}_{49}$ nanowire arrays as demonstrated in this section lead to low capacity high power safe batteries. However, the low capacity is still a major drawback especially for mobile and transport applications which led to the study of other metal oxides as potential anode materials. In this context, $\mathrm{MoO}_{3}$ nanowires have the right balance with high theoretical capacity and energy density. The next section is hence, focused on the electrochemical properties of these nanowire arrays to determine their potential as anode materials.

\subsection{Characterization of $\mathrm{MoO}_{3-\mathrm{x}}$ arrays}

\subsubsection{Physical properties}

The SEM image in Figure 7.9 shows that the average diameters of the nanowires is $\sim 90 \mathrm{~nm}$ and are several microns in length. The array architecture of the nanowires is clearly visible in these images.

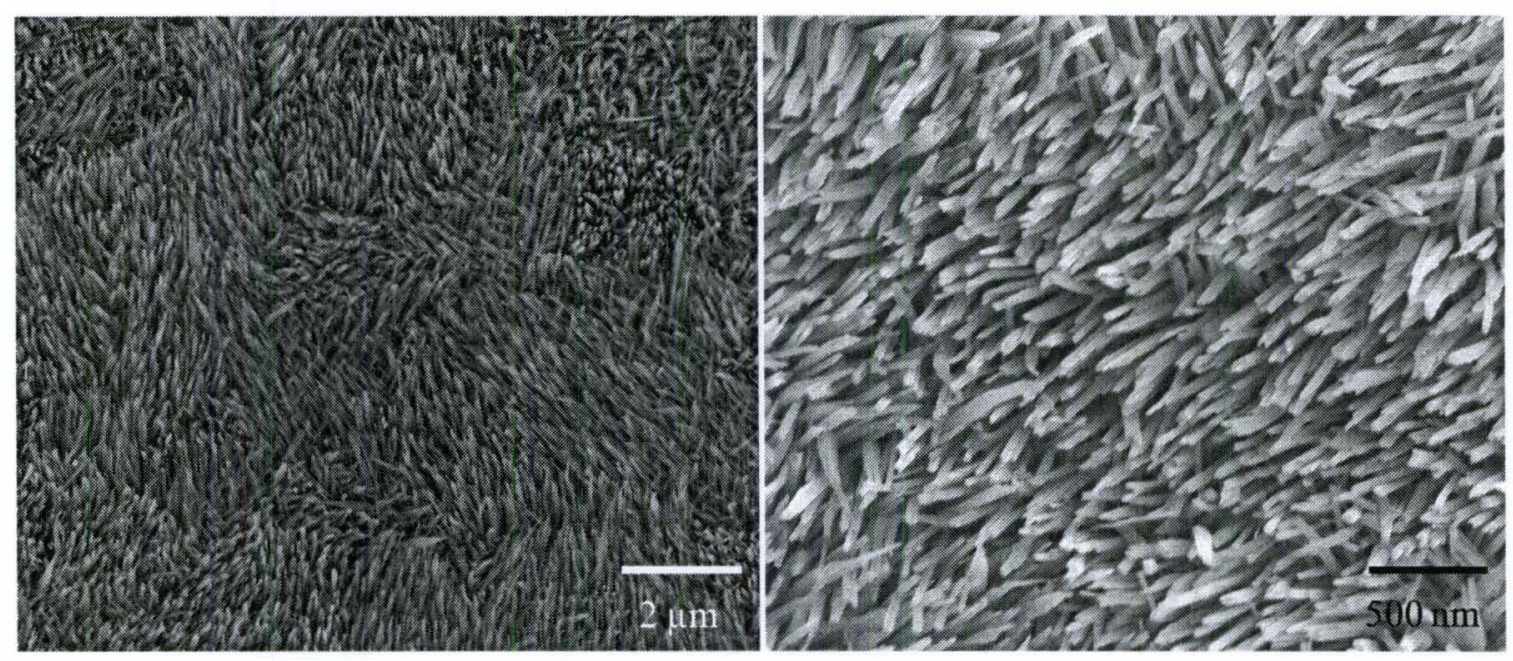

Figure 7.9: $\mathrm{SEM}$ images of as synthesized $\mathrm{Mo}_{17} \mathrm{O}_{47}$ nanowires. 


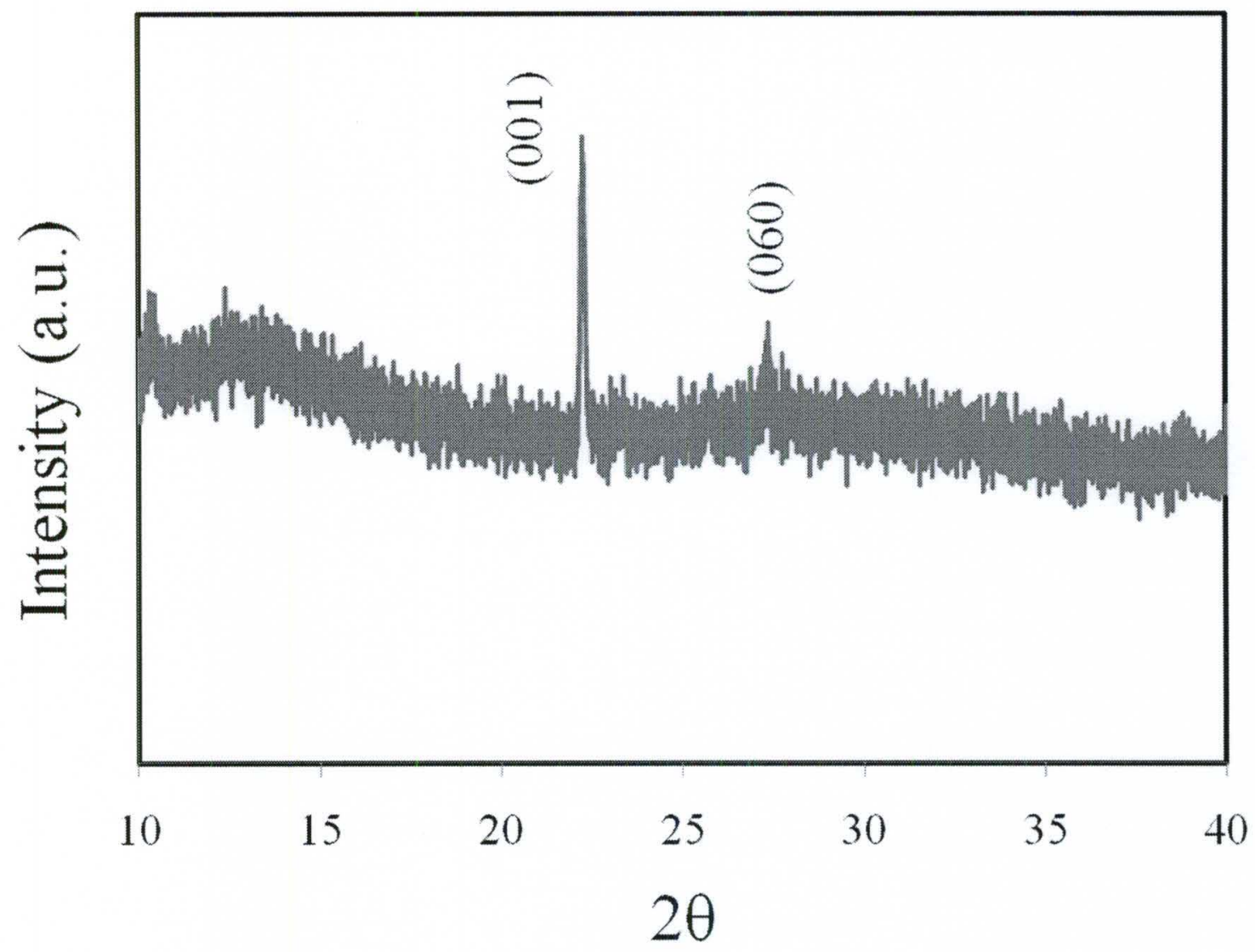

Figure 7.10: $\mathrm{XRD}$ spectrum of $\mathrm{Mo}_{17} \mathrm{O}_{47}$ nanowire array.

The as synthesized nanowire arrays are dark colored. The XRD spectrum presented in figure 7.10 indicates a oxygen deficient $\mathrm{Mo}_{17} \mathrm{O}_{47}$ phase for the nanowire $\operatorname{arrays}^{168-170}$. Oxygen deficiency renders the dark color to the array sample. Raman spectrum for these arrays is shown in Figure 7.11. The peaks at higher wavenumbers (905 and $984 \mathrm{~cm}^{-1}$ ) are as a result of the Mo-O vibrations whereas the peaks at $830 \mathrm{~cm}^{-1}$ is due to the vibrations of $\mathrm{Mo}-\mathrm{O}_{2}$ bonds. In addition, $\mathrm{Mo}-\mathrm{O}_{3}$ vibrations result in the $799 \mathrm{~cm}^{-1}$ peak $^{168-170}$. The presence of multiple peaks indicates complex interactions of Mo an O atoms further iterating the fact that the nanowire arrays are oxygen deficient giving rise to $\mathrm{MoO}_{3-\mathrm{x}}$ nanowire arrays. 


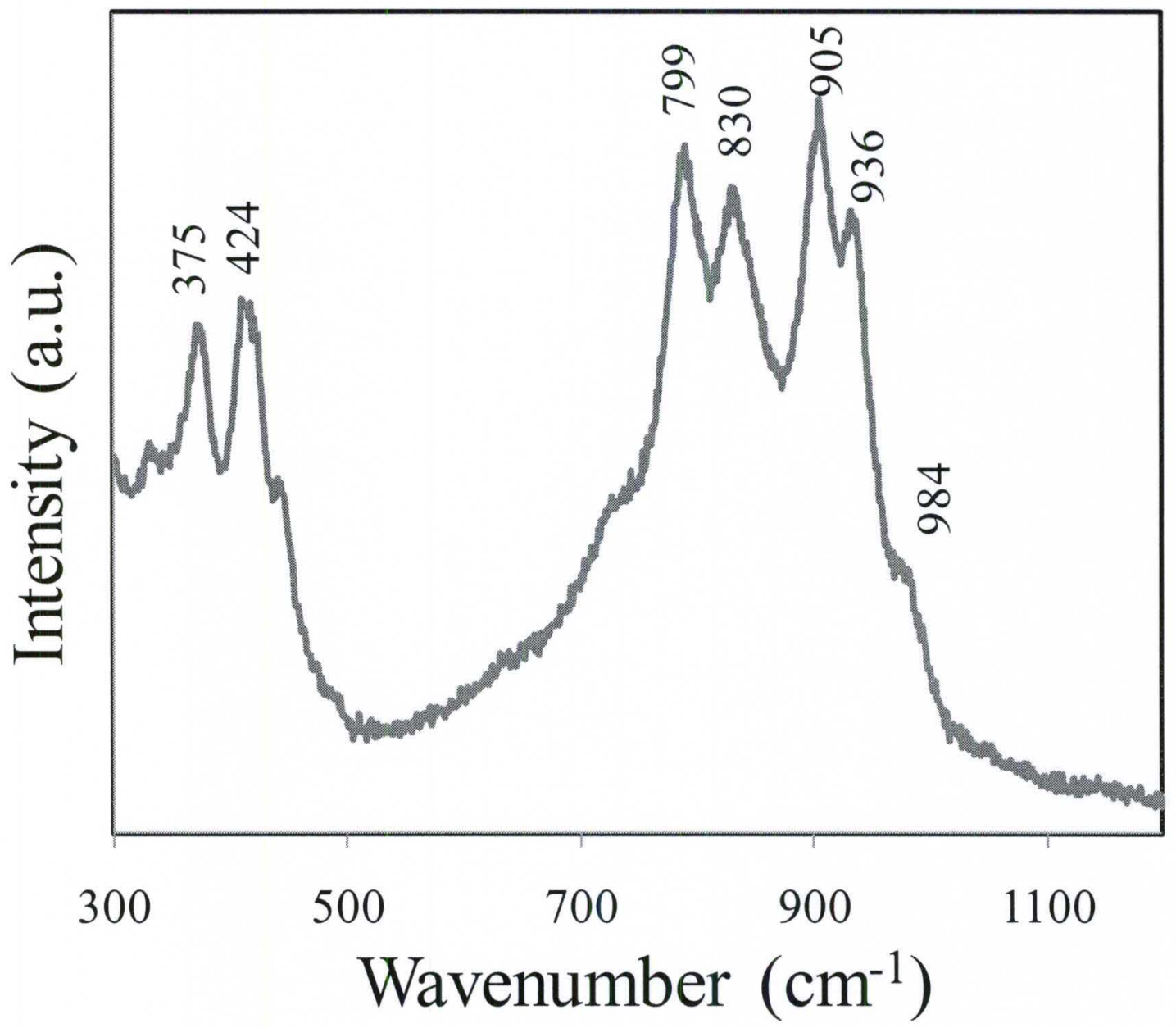

Figure 7.11: Raman spectrum of $\mathrm{Mo}_{17} \mathrm{O}_{47}$ nanowire arrays with multiple peak positions.

\subsubsection{Electrochemical properties}

$\mathrm{MoO}_{3}$ nanowires were cycled between $100 \mathrm{mV}$ and $3.5 \mathrm{~V}$. The capacity retention of the nanowires is $\sim 630 \mathrm{mAhg}^{-1}$ for up to 25 cycles and is presented in Figure 7.12. The initial discharge capacity is $\sim 770 \mathrm{mAhg}^{-1}$ and corresponds to $4.1 \mathrm{Li}^{+}$ions per an atom of Mo. The second discharge capacity is $\sim 635 \mathrm{mAhg}^{-1}$ and remains constant up to 20 cycles at a current density of $50 \mathrm{mAhg}^{-1}$. $\mathrm{MoO}_{3}$ electrodes in the present study have been cycled only down to $100 \mathrm{mV}$, much higher than the usual $10 \mathrm{mV}$. Although the lower voltages 
result in higher capacities, it comes at the expense of lithium plating on to the electrodes. The present study thus prevents the plating even at high rates due to higher voltage operation.

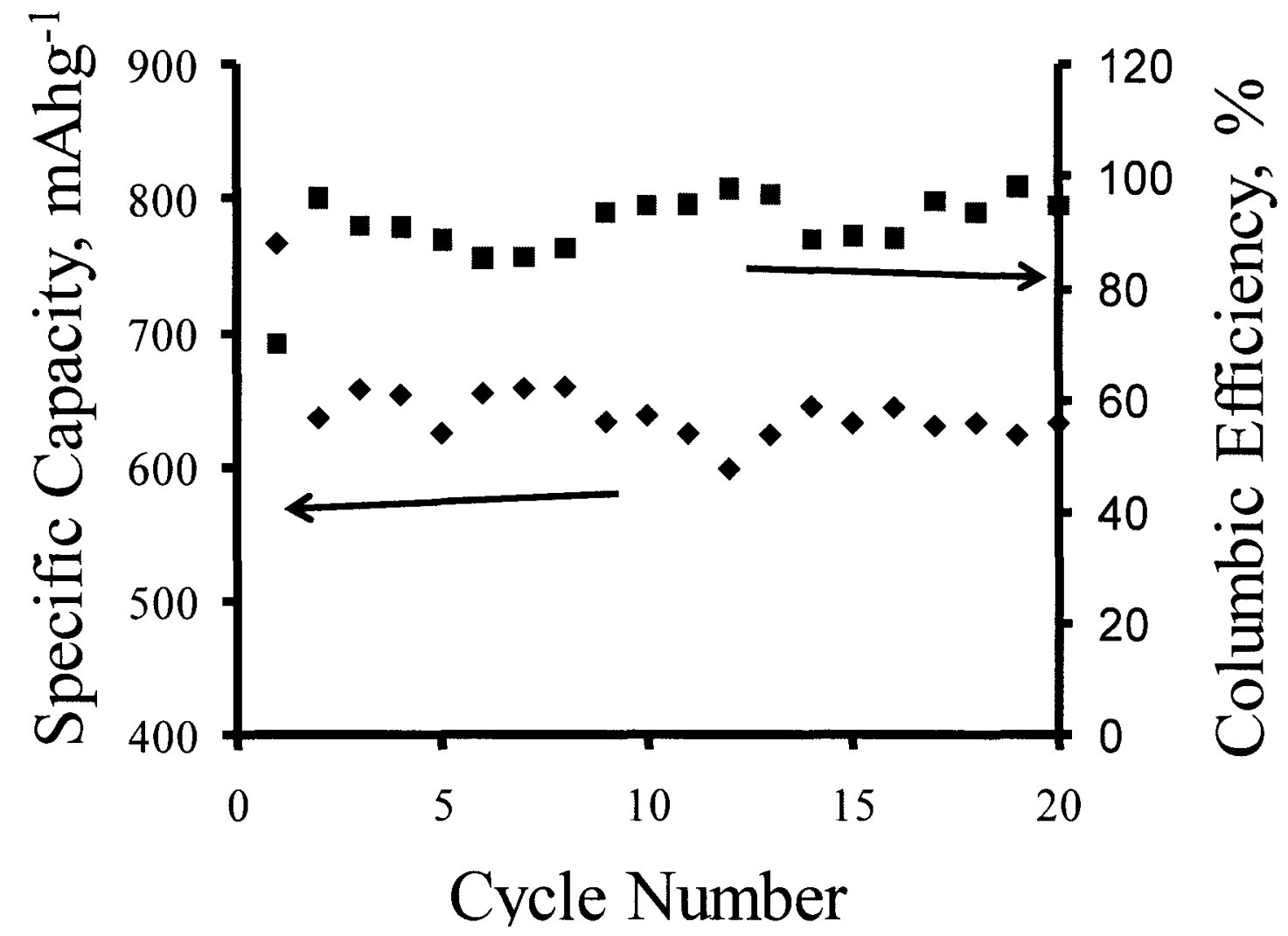

Figure 7.12: Cycling performance of $\mathrm{MO}_{3-\mathrm{x}}$ nanowire arrays. The secondary axis indicates the columbic efficiency.

The first cycle irreversible capacity loss is $176 \mathrm{mAhg}^{-1}$ corresponding to a columbic efficiency of $70 \%$ which is low in transition metal oxide systems. The columbic efficiency data presented on the secondary axis of Figure 7.12 clearly indicates that the efficiency is over $90 \%$ for the subsequent cycles. The lithiation in $\mathrm{MoO}_{3}$ is believed to 
take place in two stages: Stage $\mathrm{I}$ is up to a potential of $1.5 \mathrm{~V}$. During this stage, $\mathrm{Li}$ intercalates with $\mathrm{MoO}_{3}$ as follows ${ }^{171,172}$ :

$$
\mathrm{MoO}_{3}+\mathrm{xLi}^{+}+\mathrm{xe}^{-} \rightarrow \mathrm{Li}_{\mathrm{x}} \mathrm{MoO}_{3}
$$

The lithium content in this solid solution ranges between $1-1.5$ up to a potential of 1.5 V. The high lithium content is accommodated in the interlayer spacing between octahedron Mo-O layers and intralayers. Most of the $\mathrm{Li}$ ions intercalated at these potentials can subsequently be extracted from the material leading to reversibility of the reaction.

Lithium ion intercalation during stage II corresponds to potentials below $0.5 \mathrm{~V}$ and occurs by the following mechanism ${ }^{173,174}$ :

$$
\mathrm{Li}_{\mathrm{x}} \mathrm{MoO}_{3}+\mathrm{yLi}^{+}+\mathrm{ye}^{-} \rightarrow \mathrm{Mo}+3 \mathrm{Li}_{2} \mathrm{O}
$$

In the lower voltage range, lithium reacts with the solid solution to consequently form metal and $\mathrm{Li}_{2} \mathrm{O}$. The metal particles help in reversibly decomposing $\mathrm{Li}_{2} \mathrm{O}$ and form $\mathrm{MoO}_{3-\mathrm{x}}$. Discharge curves presented in Figure 7.13 indicate a curve which is continuous and smooth until $0.7 \mathrm{~V}$ followed by a plateau unlike the bulk material performance devoid of plateaus. $\mathrm{MoO}_{3}$ nanoparticles have been known to show similar two-stage behavior; however, the curves in that case are continuous and have much lower capacity retention below $0.7 \mathrm{~V}$ which makes these nanowire arrays practically applicable. This reversibility of $\mathrm{Li}_{2} \mathrm{O}$ is a primary reason for the material to exhibit good columbic efficiency. During the extraction reaction, the Mo metal is re-oxidized by the decomposition of $\mathrm{Li}_{2} \mathrm{O}$ which is indicated by the charge curve voltage slope shown in Figure 7.14. 


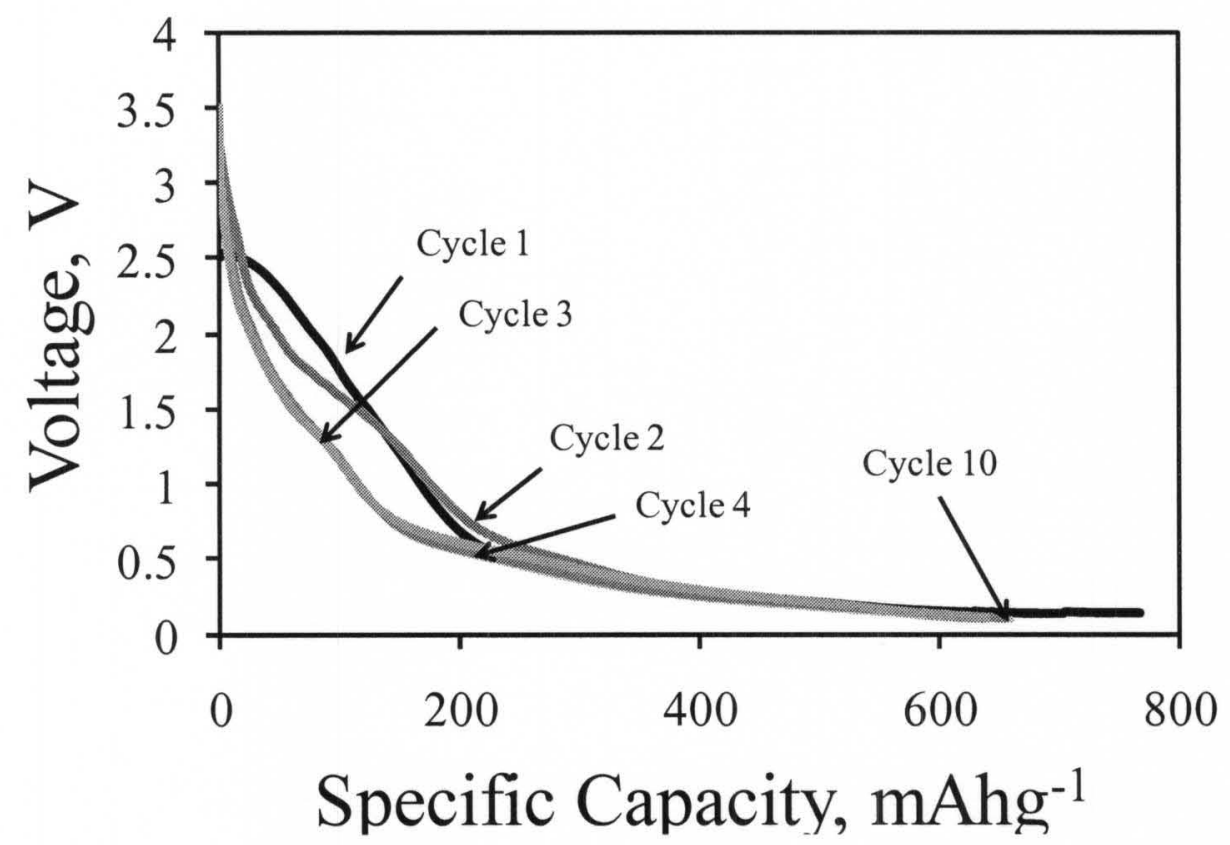

Figure 7.13: Discharge voltage profiles of $\mathrm{MO}_{3-\mathrm{x}}$ arrays for up to 10 cycles. The discharge voltage profiles are nearly same after the $3^{\text {rd }}$ cycle.

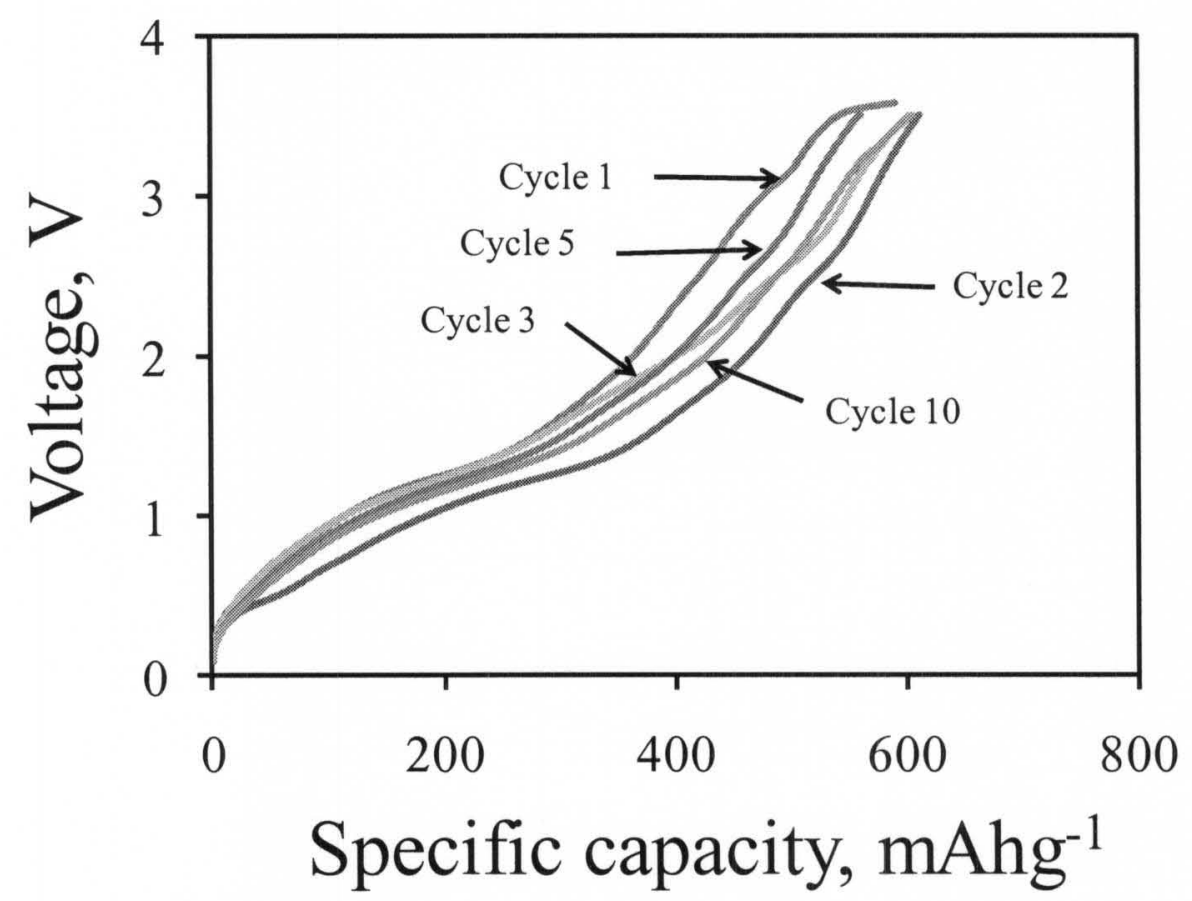

Figure 7.14: Charge voltage profiles of $\mathrm{MO}_{3-\mathrm{x}}$ arrays for up to 10 cycles showing similar profiles for all the cycles. 
Cyclic voltammetry (CV) curves obtained at scan rate of $0.5 \mathrm{mVs}^{-1}$ are presented in Figure 7.15. CV of the first discharge cycle indicates peaks at two potentials, a broad peak at $2.3 \mathrm{~V}$ and a peak at $\sim 1.5 \mathrm{~V}$. These peaks correspond to the formation of solid solution as indicated by Equation 1 with more than $1 \mathrm{Li}$ per $\mathrm{MoO}_{3}$ molecule. The first cycle discharge data indicates capacity of $\sim 200 \mathrm{mAhg}^{-1}$ at potentials over $1.5 \mathrm{~V}$ which corresponds to $1.1 \mathrm{Li}$ per $\mathrm{MoO}_{3}$ atom, consistent with the $\mathrm{CV}$ data for the first discharge cycle. However, the peaks pertaining to low potential intercalation are absent in the CVs presented here. $\mathrm{CV}$ curves until 5 cycles show three distinct peaks at $2.3 \mathrm{~V}, 2.1 \mathrm{~V}$ and 1.7 $\mathrm{V}$ following lithium intercalation into $\mathrm{MoO}_{3}$ as indicated by Equation 7.2. The peaks during the charge cycle are absent probably due to complex reaction kinetics.

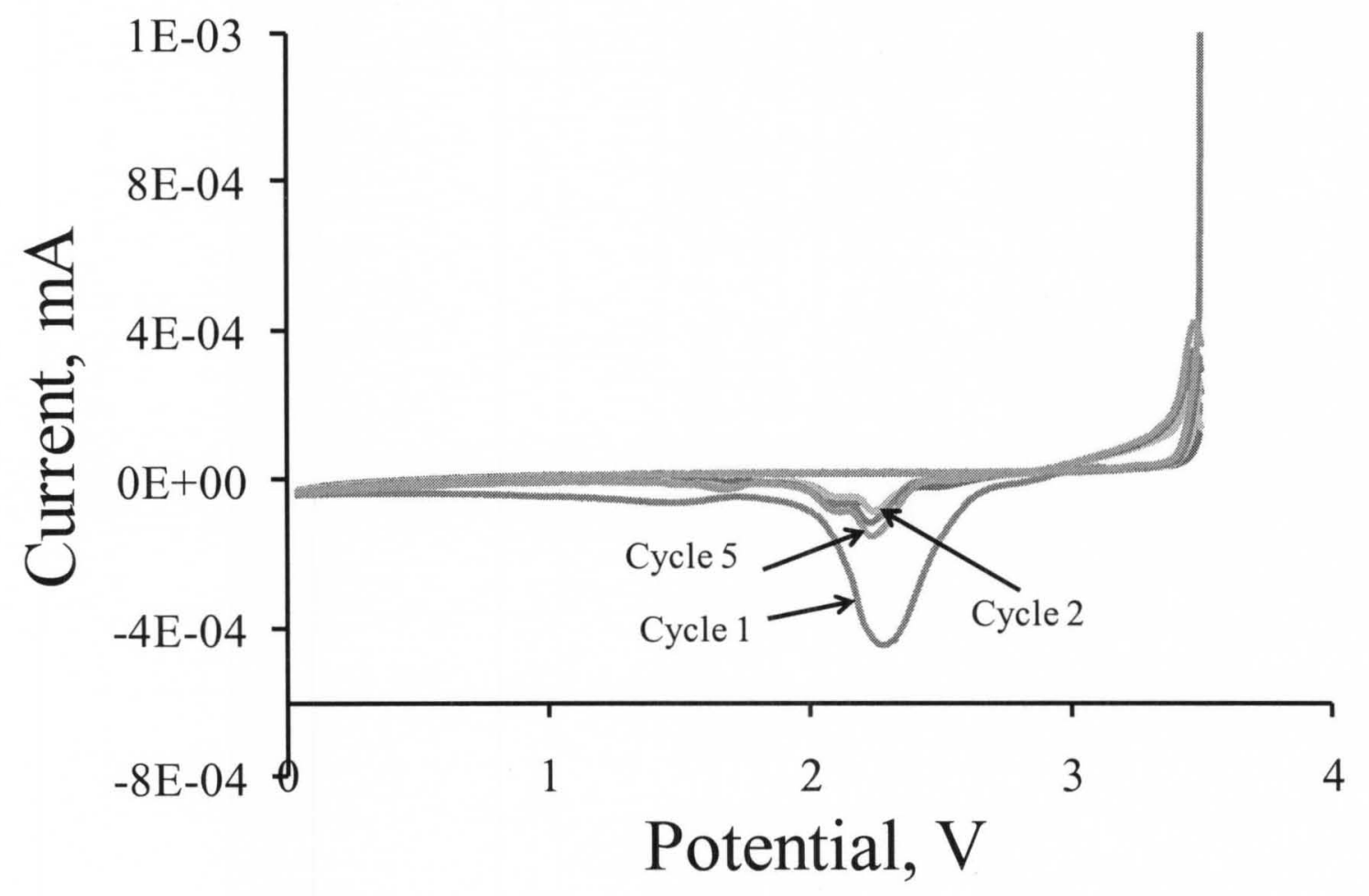

Figure 7.15: CVs of $\mathrm{MO}_{3-\mathrm{x}}$ arrays for up to 5 cycles showing peaks pertaining to $\mathrm{Li}$ penetration. 
The differential capacity curves are shown in Figures 7.16 and 7.17. The first cycle differential capacity (DC) curve shows a small peak in the higher potential region above $1.5 \mathrm{~V}$ and multiple peaks in the low potential region below $0.5 \mathrm{~V}$. These peaks indicate the $\mathrm{Li}$ intercalation by a mechanism shown in Equation 7.3. DC curves for the next few cycles also indicate peaks above $1.5 \mathrm{~V}$ and below $0.5 \mathrm{~V}$, further confirming that lithium intercalates by both mechanisms in Equations 7.2 and 7.3. These peaks appear until the $10^{\text {th }}$ cycle as indicated in Figure 7.16 showing excellent reversibility of the electrode ${ }^{102}$. However, the absence of lower voltage lithiation peaks in CVs indicates that the reaction kinetics might be complex.

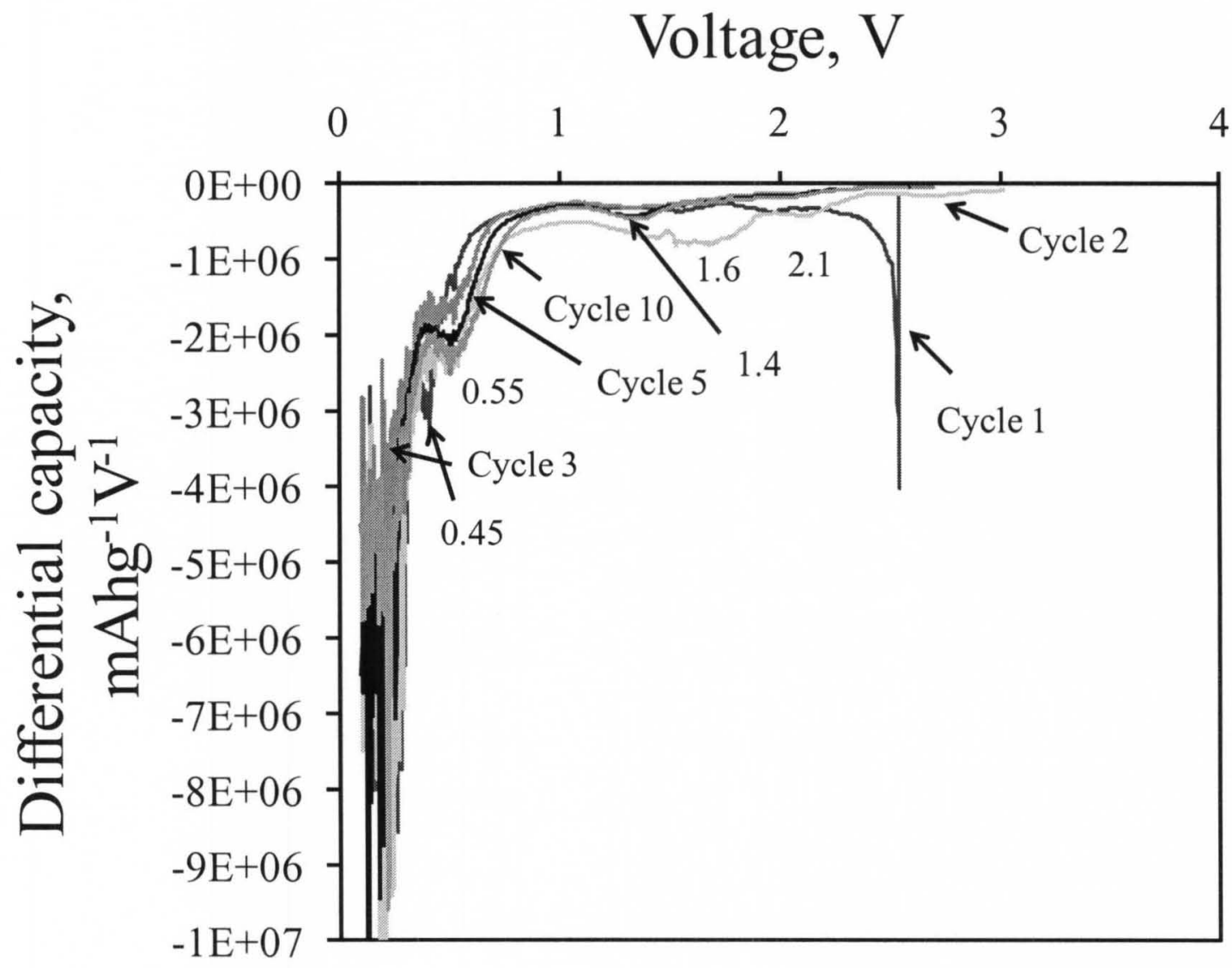

Figure 7.16: DC curves of discharge cycles for the first 10 cycles indicating lithiation in 2 stages. 
Figure 7.17 is the DC curves for the charge cycle of these arrays. The charge cycles clearly indicate peaks around $0.5 \mathrm{~V}, 1.2 \mathrm{~V}, 1.8 \mathrm{~V}$ and $2.6 \mathrm{~V}$ indicating that the delithiation occurs in two stages as illustrated in Equations 7.2 and 7.3. These peaks are clearly evident in all the cycles indicating excellent reversibility of the nanowire arrays.

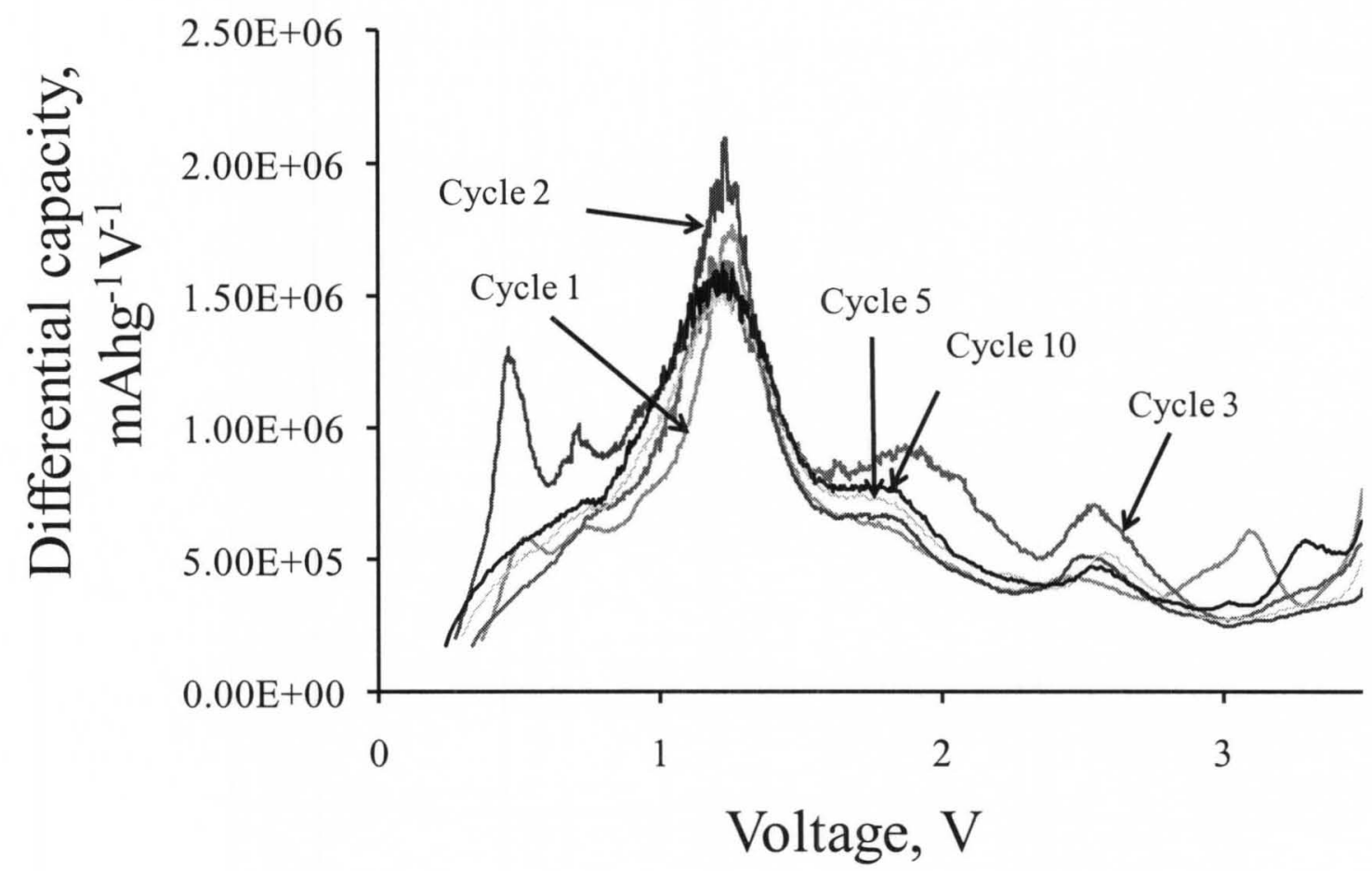

Figure 7.17: DC curves of the charge cycles for the first 10 cycles indicating de-lithiation in 2 stages.

A complete reduction of $\mathrm{MoO}_{3}$ requires $6 \mathrm{Li}^{+}$ions per Mo. The first cycle capacity of $\sim 770 \mathrm{mAhg}^{-1}$ corresponds to $4.1 \mathrm{Li}^{+}$ions per one Mo atom, of which $1.1 \mathrm{Li}^{+}$ ions are intercalated as indicated in Equation 1. The remaining $3 \mathrm{Li}^{+}$ions intercalate with the solid solution by the mechanism indicated in Equation 2. This indicates an incomplete conversion to $\mathrm{Li}_{2} \mathrm{O}+$ Mo. Further cycles indicate capacities close to $\sim 650 \mathrm{mAhg}^{-1}$ corresponding to $3.4 \mathrm{Li}^{+} / \mathrm{Mo}$. However, the data over next few cycles shows interesting 
behavior, i.e., the addition of lithium (Equation 7.2) accounts to less than $100 \mathrm{mAhg}^{-1}$ that corresponds to only $\sim 0.6 \mathrm{Li}^{+} / \mathrm{Mo}$. The alloying reaction (Equation 7.3 ) accounts for $\sim 500 \mathrm{mAhg}^{-1}$ capacity equivalent to $2.8 \mathrm{Li}^{+} / \mathrm{Mo}$, indicating a good reversibility of the alloying reaction. The data pertaining to capacity retention between $0.7 \mathrm{~V}$ and $0.1 \mathrm{~V}$ is presented in Figure 7.18. $\mathrm{MoO}_{3-\mathrm{x}}$ nanowire arrays are believed to have better electron conduction pathways compared to their nanoparticle counterparts and well dispersed metal nanoparticles in the nanowire matrix can lead to better intercalation properties of these materials leading to higher capacity retention in the lower potential range.

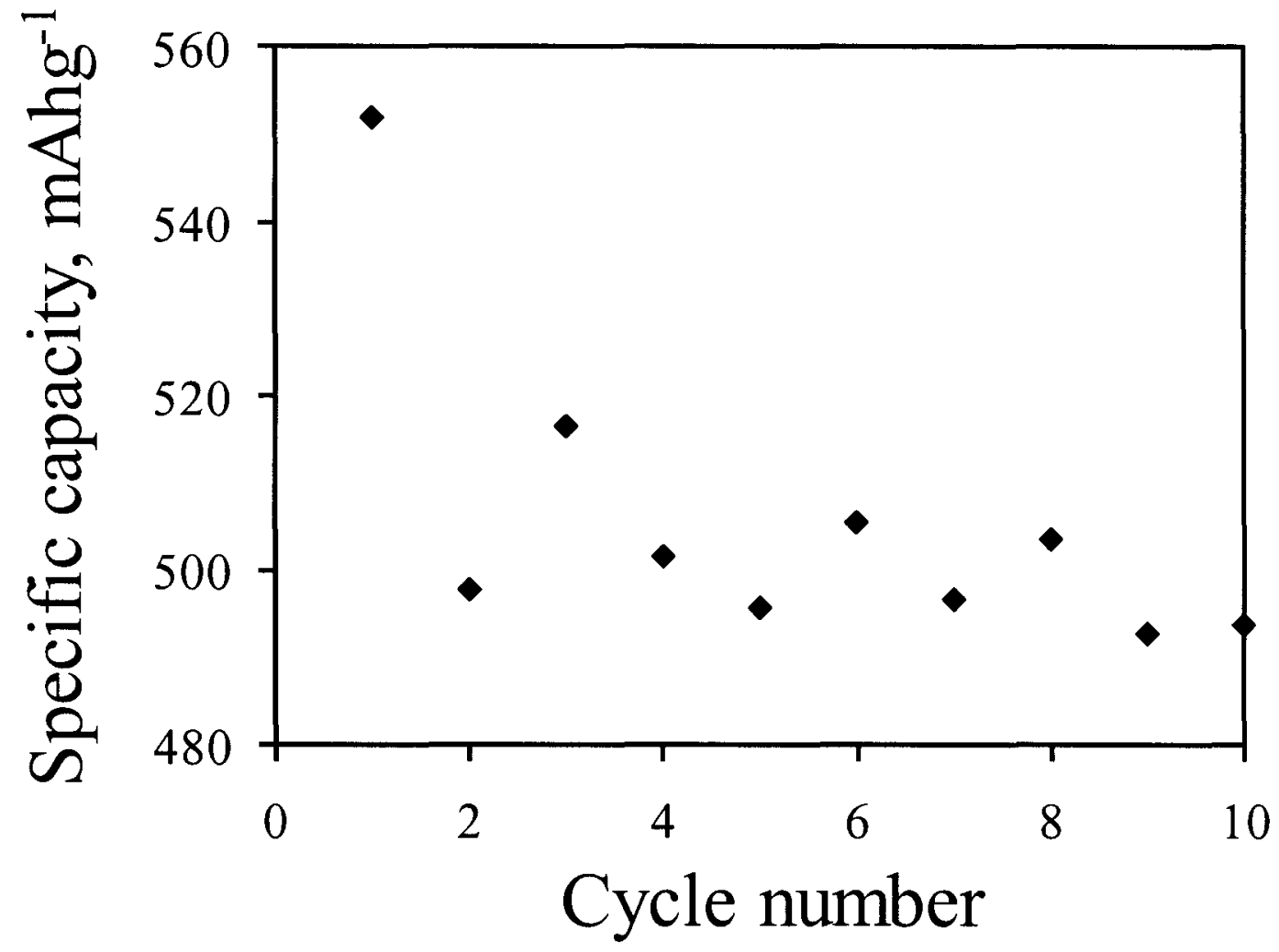

Figure 7.18: Specific capacity retention of $\mathrm{MoO}_{3-\mathrm{x}}$ arrays in the potential range of $0.7-$ $0.1 \mathrm{~V}$. 
The performance of nanowire arrays at various charge and discharge rates has shown capacity retentions of $632 \mathrm{mAhg}^{-1}$ and $590 \mathrm{mAhg}^{-1}$ at current densities of 50 $\mathrm{mAhg}^{-1}$ and $100 \mathrm{mAg}^{-1}$ respectively as shown in Figure 7.19. It is to be noted that a capacity of $685 \mathrm{mAhg}^{-1}$ at $25 \mathrm{mAg}^{-1}$ is obtained on switching from the high rate to low rate. These nanowire arrays can hence, be used as base materials to develop hybrid architecture materials with materials such as $\mathrm{Si}$ which can significantly enhance the capacity retention and stability. $\mathrm{Si}$ is particularly interesting as the intercalation potentials of $\mathrm{Si}$ and $\mathrm{MoO}_{3}$ match well at around $0.7 \mathrm{~V}$ making them ideal hybrid materials.

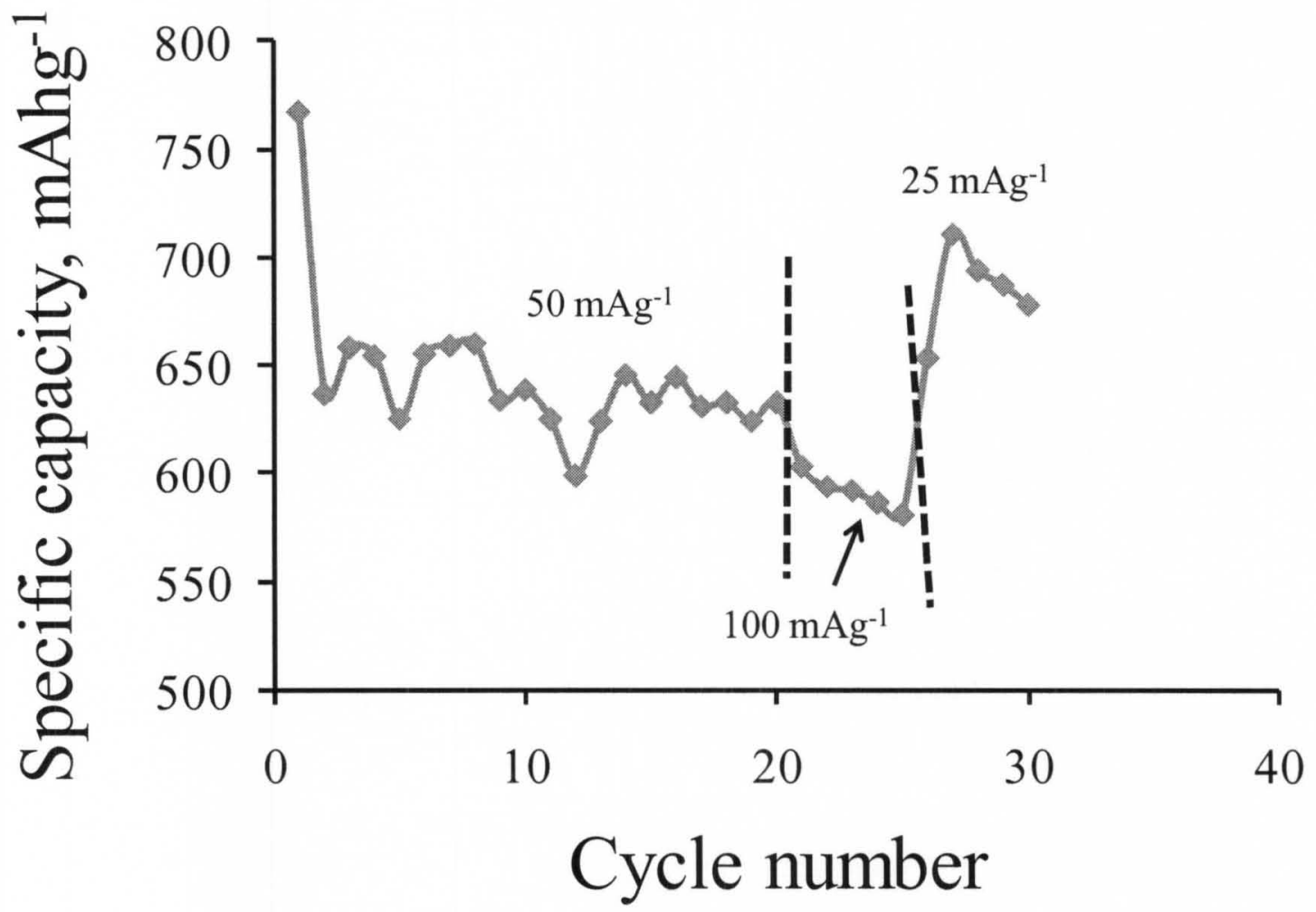

Figure 7.19: Rate performance of $\mathrm{MoO}_{3-\mathrm{x}}$ arrays indicating stable capacity retention. 
In order to determine whether one can improve the capacity retention using silicon coatings, the $\mathrm{MoO}_{3-\mathrm{x}}$ nanowire arrays were coated with thin silicon coating and tested. The TEM image presented in Figure 7.20a shows Si deposition on the surface which has a thickness of $10 \mathrm{~nm}$. However, line scan of the image shows the presence of low oxygen amounts in silicon. In theory, about $10 \%$ silicon is present in these samples (by weight) which correspond to a theoretical capacity for the entire samples is about of $\sim 1370 \mathrm{mAhg}^{-1}$, compared to $1116 \mathrm{mAhg}^{-1}$ for pure $\mathrm{MoO}_{3}$ nanowire array sample. The specific capacity data presented in Figure $7.20 \mathrm{~b}$ shows that the hybrid architecture of $\mathrm{Si} / \mathrm{MoO}_{3-\mathrm{x}}$ nanowires shows initial capacity of $1065 \mathrm{mAhg}^{-1}$ with a capacity retention of $\sim 780 \mathrm{mAhg}^{-1}$ for up to 15 cycles. Most importantly, the cycling data over 15 cycles represent initial results regarding the stability of silicon coated $\mathrm{MoO}_{3}$ nanowire arrays. The capacity of $\mathrm{MoO}_{3}$ nanowire array samples can easily be improved by coating them more silicon. In a typical $\mathrm{MoO}_{3}$ nanowire array sample, there is about $10^{9} / \mathrm{cm} 2$ density of $\mathrm{MoO}_{3-\mathrm{x}}$ nanowires which leaves about $50 \%$ volume for including silicon to obtain a 5050 mixture of $\mathrm{Si}$ and $\mathrm{MoO}_{3-\mathrm{x}}$ hybrid material. The theoretical capacity for such 50:50 $\mathrm{MoO}_{3} \mathrm{NW}$ array and silicon layers is about $2650 \mathrm{mAhg}^{-1}$. The rate performance presented in Figure $7.20 \mathrm{c}$ shows a stable performance of $\sim 580 \mathrm{mAhg}^{-1}$ at $200 \mathrm{mAg}^{-1}$. This study shows that these hybrid architectures are promising materials and further fine tuning is necessary to develop high capacity and high rate capable anode materials based on silicon. 
(a)
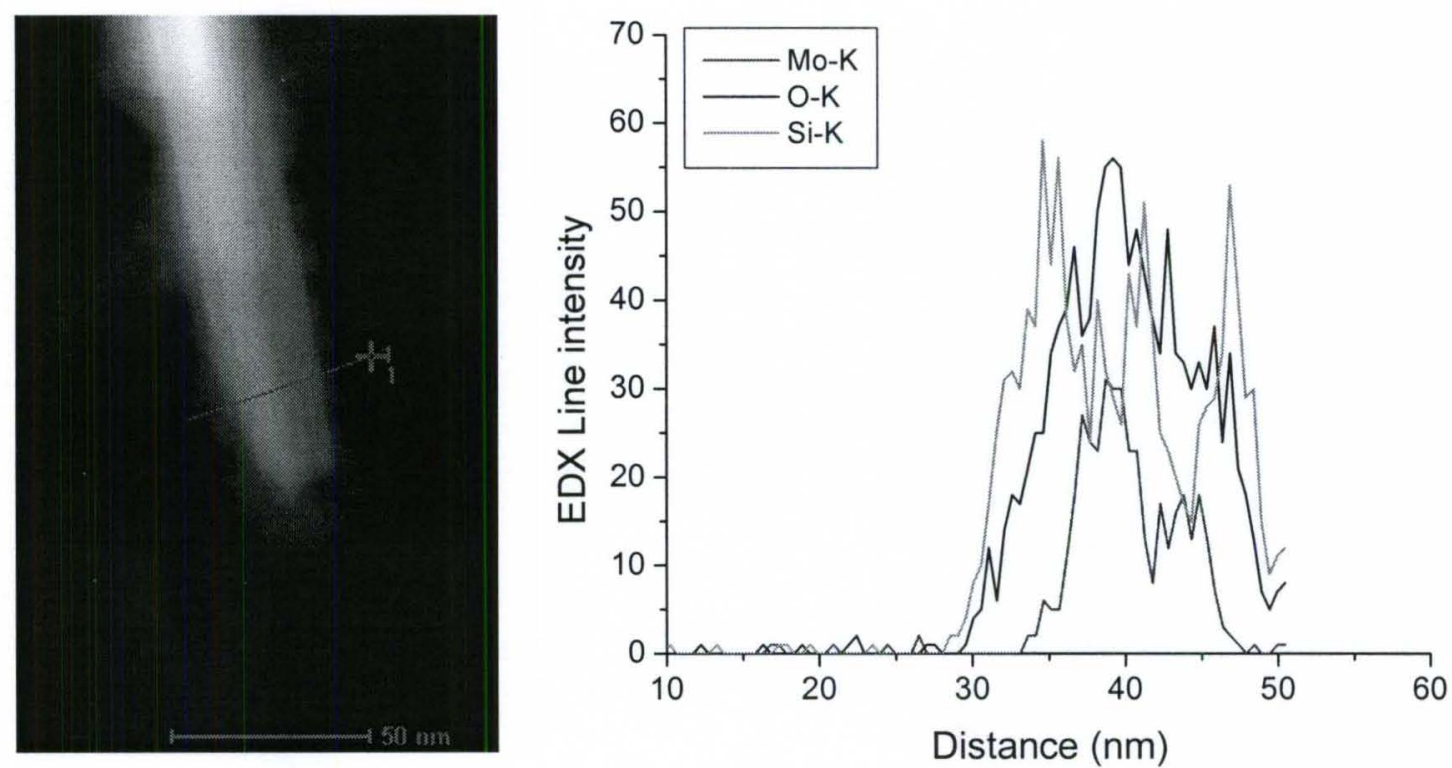

(b)

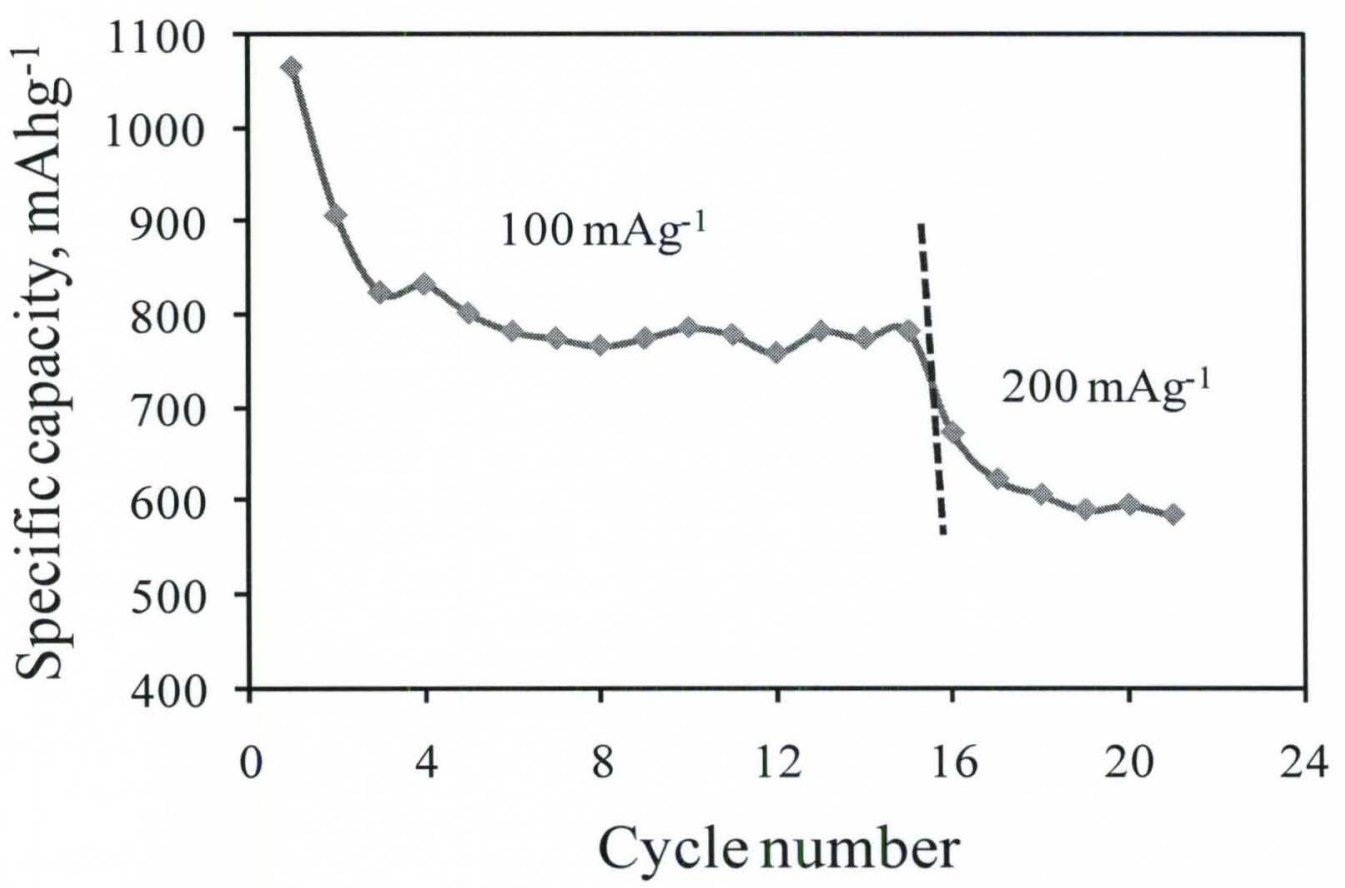

Figure 7.20: $\mathrm{Si} / \mathrm{MoO}_{3-\mathrm{x}}$ hybrid architectures (a) Line scan in a TEM showing $10 \mathrm{~nm}$ silicon coating on $\mathrm{MoO}_{3-\mathrm{x}}$ nanowires. (b) Specific capacity of hybrid materials with capacity retentions of 780 and $\sim 580 \mathrm{mAhg}^{-1}$ at rates of 100 and $200 \mathrm{mAg}^{-1}$, respectively. 


\subsection{Summary}

Metal oxide nanowire arrays synthesized directly on to conducting substrates provide direct conduction pathways between the material and the substrate without the use of binder and conducting additive which can improve efficiency in the fabrication of anodes. In this context, $\mathrm{W}_{18} \mathrm{O}_{49}$ nanowires are tested as potential anode materials with capacity retention of $\sim 175 \mathrm{mAhg}^{-1}$ for up to 20 cycles. The post-lithiated SEM images show a stable nanowire architecture indicating good reversibility of the material. Even though the material has high energy density, the capacity obtained is limited and prompted our interest in other metal oxides, primarily molybdenum oxide. In this study, $\mathrm{MoO}_{3-\mathrm{x}}$ nanowire arrays are shown to retain capacity of $\sim 630 \mathrm{mAhg}^{-1}$ for up to 20 cycles at a current density of $50 \mathrm{mAg}^{-1}$. These nanowire arrays undergo a 2 stage lithiation/delithiation mechanism which happens between $1.5 \mathrm{~V}$ and $0.5 \mathrm{~V}$ leading to a retention of 3.4 Li ions per Mo. $\mathrm{MoO}_{3-\mathrm{x}}$ nanowire arrays show good capacity retention of $\sim 500 \mathrm{mAhg}^{-1}$ below $0.7 \mathrm{~V}$ indicating viable practical applicability of the material. In addition, $\mathrm{Si} / \mathrm{MoO}_{3-\mathrm{x}}$ hybrid nanowires synthesized by the direct deposition of $\mathrm{Si}$ on $\mathrm{MoO}_{3-\mathrm{x}}$ nanowire arrays have shown higher capacity retentions of $\sim 780 \mathrm{mAhg}^{-1}$ paving ways for high capacity stable anode materials. 


\section{CHAPTER 8}

\section{CONCLUSIONS}

This study investigates the nanowire based architectures as anode materials for LIBs. The dissertation is primarily divided into four parts. The first part of the dissertation focuses on the use of pure phase $\mathrm{SnO}_{2}$ nanowires and mixed $\mathrm{Sn}$ clusters with $\mathrm{SnO}_{2}$ nanowires as potential anode materials. The second part is concentrated on the effort to modify the nanowire architectures to form Sn-nanocluster-covered $\mathrm{SnO}_{2}$ nanowires which show enhanced lithiation and de-lithiation properties as suggested by the scientific aspects of the study. In the next part, thin walled carbon microtubes have been shown to sustain high rates with low irreversible capacity and high columbic efficiencies. These materials are particularly attractive as a conducting base in hybrid architectured materials. Finally, metal oxide nanowire arrays grown directly on conducting substrates which eliminate the need for a conducting material and polymeric binder show high capacity retention with minimal loss in capacity with cycling.

\section{1 $\mathrm{SnO}_{2}$ nanowire based electrodes}

Electrodes were made using pure phase $\mathrm{SnO}_{2}$ nanowires and micron sized $\mathrm{Sn}$ clusters mixed with pure phase $\mathrm{SnO}_{2}$ nanowires. The most important conclusions of this study are: 
- Pure phase $\mathrm{SnO}_{2}$ nanowires show a first cycle discharge capacity of $2470 \mathrm{mAhg}^{-1}$ due to the high surface area of the material. However, the second cycle discharge capacity is $\sim 1030 \mathrm{mAhg}^{-1}$ indicating capacity loss, which is attributed to the formation of amorphous $\mathrm{Li}_{2} \mathrm{O}$ and SEI layer. However, the capacity degradation occurs over the next 15 cycles to $\sim 170 \mathrm{mAhg}^{-1}$.

- In order to overcome the capacity degradation problems, a new architecture of mixed $\mathrm{Sn}$ clusters with $\mathrm{SnO}_{2}$ nanowires is proposed. These architectures are believed to disperse the $\mathrm{Sn}$ particulates well which results in higher available surface area for lithiation with cycling.

- Mixed $\mathrm{Sn}$ clusters with $\mathrm{SnO}_{2}$ nanowires show high initial capacity with a capacity retention of $\sim 490 \mathrm{mAhg}^{-1}$ for up to 40 cycles. However, the specific capacity is significantly lower than the theoretical capacity of Sn (991 mAhg $\left.{ }^{-1}\right)$ which led to the design of hybrid architectures.

\subsection{Hybrid architectured materials}

A unique and simple generic design of Sn-nanocluster-covered $\mathrm{SnO}_{2}$ nanowires has been proposed to overcome capacity degradation. The outcomes of this study include:

- Hybrid nanowires show high initial capacity with a reversible capacity of $\sim 810$ $\mathrm{mAhg}^{-1}$ for up to 100 cycles, which is the highest reported to date for $\mathrm{SnO}_{2}$ based materials. In addition, the materials show columbic efficiency of over $98 \%$ indicating practical applicability of the material.

- Excellent stability of the material is attributed to two phenomena. Cyclic voltammetry studies indicate that de-lithiation proceeds by a stage-wise 
mechanism of lithium extraction which in the case of pure $\mathrm{SnO}_{2}$ nanowires proceeds by a single stage mechanism. The stage-wise mechanism leads to maximum lithium extraction from the material.

- Additionally, post lithiation analysis using XRD indicates that $S n$ nanoclusters on the nanowire periphery undergo volume expansion but remain intact. The underlying $\mathrm{SnO}_{2}$ nanowire reversibly forms $\mathrm{SnO}$ phase which gives rise to electronic conductivity.

- De-lithiation characteristics show stage-wise lithium extraction in nanoscale Sn particles whereas the micron scale Sn particles show a single stage extraction. Micron scale Sn particles show a concentration gradient along the spherical particle due to diffusion control. However, the lithium extraction is controlled by the kinetics in nanoscale Sn materials resulting in lithium removal from the particles in a stage-wise manner.

\subsection{Carbon microtube electrodes}

Thin walled CMTs with wall thickness of $\sim 50 \mathrm{~nm}$ were synthesized on large area substrates for the first time. The most important conclusions of this study are:

- CMTs have shown a capacity of $450 \mathrm{mAhg}^{-1}$ after 20 cycles, $\sim 1.5$ times higher than MWCNTs. The average charge capacity loss was reported to be $1.3 \%$, which is quite low for carbon based materials.

- CMTs are able to withstand high rates with capacity retention of $135 \mathrm{mAhg}^{-1}$ at a rate of $5 \mathrm{C}$ and then reversibly achieving the original capacity at lower rates. This high rate performance is on par, with other carbon based materials but the synthesis of CMTs for practical purposes is quite economical. 
- Analysis has shown that graphite nanodomains in the walls and open ends of CMTs provide channels for $\mathrm{Li}$ intercalation and de-intercalation. Post-lithiated samples show that the graphite disorderliness increases with cycling due to the volume expansion but the microtube structure remains firm.

\subsection{Nanowire array electrodes}

Metal oxide nanowire arrays synthesized directly on to conducting substrates provide direct conduction pathways between the material and the substrate. Electrodes fabricated using the arrays have the following advantages:

- $\mathrm{W}_{18} \mathrm{O}_{49}$ nanowires showed capacity retention of $\sim 175 \mathrm{mAhg}^{-1}$ for up to 20 cycles. The post-lithiated SEM images show a stable nanowire architecture indicating good reversibility of the material.

- The capacity of $\mathrm{W}_{18} \mathrm{O}_{49}$ nanowires is limited which prompted us to look at molybdenum oxide. In this study, $\mathrm{MoO}_{3-\mathrm{x}}$ nanowire arrays are shown to retain capacity of $\sim 630 \mathrm{mAhg}^{-1}$ for up to 20 cycles.

- Nanowire arrays show good practical applicability because of high capacity retention below $0.7 \mathrm{~V}$ along with good rate performance. 


\section{CHAPTER 9}

\section{FUTURE RECOMMENDATIONS}

The hybrid architecture has been shown to work with a $\mathrm{Sn} / \mathrm{SnO}_{2}$ material system which showed excellent capacity and stability. This concept can be further extended to growing $\mathrm{Si}$ and $\mathrm{Ge}$ nanowires on the periphery of the $\mathrm{SnO}_{2}$ nanowires. $\mathrm{Si}$ and $\mathrm{Ge}$ with theoretical capacities of $\sim 4200 \mathrm{mAhg}^{-1}$ and $1600 \mathrm{mAhg}^{-1}$ are interesting materials. In addition, the $\mathrm{Li}^{+}$diffusion rates inside $\mathrm{Ge}$ are quite high which makes them attractive for high rate charge/discharge applications. In this proposed design, the base is can be $\mathrm{SnO}_{2}$ nanowires/CMTs $/ \mathrm{MoO}_{3}$ nanowires. The Sn nanoclusters on the surface of the $\mathrm{Sn} / \mathrm{SnO}{ }_{2}$ hybrid architectures can be used as catalysts in the synthesis of Si and Ge nanowires.

Synthesis and testing of one dimensional nanowire materials as cathode materials for battery applications is limited and is quite interesting. Lithium iron phosphate $\left.(\mathrm{LiFePO})_{4}\right)$ nanowires and manganese oxide $\left(\mathrm{MnO}_{2}\right)$ nanowires are two interesting materials that are stable cathodes. In addition to studying the material performance, developing hybrid architecture materials can improve the performance.

In principle, 3-dimensional electrodes are the ideal candidates for $\mathrm{Li}$-ion batteries because the cathode, anode and the electrolyte are all packed in one compact structure eliminating the need for separate compartments and the separators which add extra weight to the batteries. In this proposed design, we have the anode materials i.e. nanowires/hybrid architectures synthesized directly on a stainless steel substrate in an 
array fashion. The array is then coated uniformly with a solid polymer electrolyte (SPE) using liquid phase techniques. The cathode material is then deposited directly on to the SPE. The cathode can also be an array of nanowires which can fit perfectly into the SPE coated anode thus, having high packing density. 3-dimensional architectures thus, increase the density of the overall electrode and eliminate the need to use liquid electrolytes. 


\section{REFERENCES}

1. Government, U. S. Energy Information Administration, U. S. Department of Energy, International Energy Outlook 2010, July 2010.

2. Bruce, P. G.; Scrosati, B.; and Tarascon, J. M., Nanomaterials for Rechargeable Lithium Batteries. Angewandte Chemie International Editon 2008, 47, 2930.

3. Pillot, C., Present and future market situation for batteries, The International Power Supply Conference and Exhibition 2009.

4. Wakihara, M.; Yamamoto, O., Lithium Ion Batteries: Fundamentals and Performance. Wiley-VCH, Tokyo, 1998.

5. J. M. Tarascon, J. M.; Armand, M., Issues and Challenges Facing Rechargeable Lithium Ion Batteries, Nature 2001, 414, 359.

6. Li, N. C.; Patrissi, C. J.; Che, G. L.; and Martin, C. R., Rate Capabilities of Nanostructured $\mathrm{LiMn}_{2} \mathrm{O}_{4}$ Electrodes in Aqueous Electrolyte, Journal of The Electrochemical Society 2000, 147, 2044.

7. Ohzuku, T.; Ueda, A.; Nagayama, N., Electrochemistry and Structural Chemistry of $\mathrm{LiNiO}_{2}$ for 4 Volt Secondary Lithium Cells, Journal of The Electrochemical Society 1993, 140, 1862.

8. Li, X. X.; Cheng, F. Y.; Guo, B.; Chen, J., Template-Synthesized $\mathrm{LiCoO}_{2}, \mathrm{LiMn}_{2} \mathrm{O}_{4}$, and $\mathrm{LiNi}_{0.8} \mathrm{Co}_{0.2} \mathrm{O}_{2}$ Nanotubes as Cathode Materials of Lithium Ion Batteries, Journal of Physical Chemistry B 2005, 109, 14017. 
9. Zhou, Y. K.; Li, H. L., Sol-gel template synthesis and structural properties of a highly ordered $\mathrm{LiNi}_{0.5} \mathrm{Mn}_{0.5} \mathrm{O}_{2}$ nanowire array, Journal of Materials Chemistry 2002, 12,681 .

10. Park, D. H.; Lim, S. T.; Hwang, S. J.; Yoon, C. S.; Sun, Y. K.; and Choy, J. H., LowTemperature Synthesis of $\mathrm{Li}_{\mathrm{x}} \mathrm{Mn}_{0.67} \mathrm{Ni}_{0.33} \mathrm{O}_{2}(0.2<\mathrm{x}<0.33)$ Nanowires with a Hexagonal Layered Structure, Advanced Materials, 2005, 17, 2834.

11. Howell, D., Progress Report for Energy Storage Research and Development, US DOE Office of Vehicle Technologies, 2009.

12. Henriksen, G. L.; Amine, K.; Liu, J.; Nelson, P. A., Materials Cost Evaluation Report for High-Power Li-ion HEV Batteries, Argonne National Laboratory, 2002.

13. Aricó, A. S.; Bruce, B.; Scrosati, B.; Tarascon, J. M.; Schalkwijk, W. V., Nanostructured materials for advanced energy conversion and storage devices. Nature Materials 2005, 4, 366.

14. Dobley, A.; Ngala, K.; Yang, S. F.; Zavalij, P. Y.; and Whittingham, M. S., Manganese Vanadium Oxide Nanotubes: Synthesis, Characterization, and Electrochemistry, Chemistry of Materials 2001, 13, 4382.

15. Nordlinder, S.; Edstrom, K.; Gustafsson, T., The Performance of Vanadium Oxide Nanorolls as Cathode Materials in a Rechargeable Lithium Battery, Electrochemical and Solid-State Letters 2001, 4, A129.

16. Website, Electricity Storage Association, www.electricitystorage.org, Technology comparisons. April 2009.

17. Buchmann, I., Batteries in a Portable World: A Handbook on Rechargeable Batteries for Non-Engineers. Cadex Electronics Inc., British Columbia, Canada, 2001. 
18. Whittingham, M. S.; Jacobson, A. J., Intercalation Chemistry (Materials Science and technology). Academic Press Inc., New York, 1982.

19. Mizushima, K.; Jones, P. C.; Wiseman, P. J.; Goodenough, J. B., $\mathrm{Li}_{\mathrm{x}} \mathrm{CoO}_{2}(0<\mathrm{x}<-1)$ : A new cathode material for batteries of high energy density. Materials Research Bulletin 1980, 15, 783.

20. Thackeray, M. M.; David, W. I. F.; Bruce, P. G.; Goodenough, J. B., Lithium insertion into manganese spinels. Materials Research Bulletin 1983, 18, 461.

21. Besenhard, J. O., Handbook of Battery Materials. Wiley-VCH Verlag GmbH, Weinheim (Federal Republic of Germany), 1999.

22. Tran, T.; Kinoshita, K., Lithium intercalation/deintercalation behavior of basal and edge planes of highly oriented pyrolytic graphite and graphite powder, Journal of Electroanalytical Chemistry 1995, 386, 221.

23. Winter, M.; Besenhard J. O.; Spahr, M. E.; Novák, P., Insertion Electrode Materials for Rechargeable Lithium Batteries Advanced Materials 1998, 10, 725.

24. Billaud, D.; McRae, E.; Hérold, A., Synthesis and Electrical of Lithium-Pyrographite Intercalation Compounds (Stages I, II and III), Materials Research Bulletin 1979, 14, 857.

25. Song, X. Y.; Kinoshita, K.; Tran, T. D., Microstructural Characterization of Lithiated Graphite, Journal of The Electrochemical Society 1996, 143, L120.

26. Rüdorff, W.; Hofmann, U., Intercalation of Nitric and Sulfuric Acids into Graphite, Zeitschrift für anorganische und allgemeine Chemie 1938, 238, 1.

27. Armand, M.; Touzain, P., Graphite intercalation compounds as cathode materials Material Science and Engineering 1977, 31, 319. 
28. Guerard, D.; Herold, A., Intercalation of Lithium into Graphite and Other Carbons, Carbon 1975, 13, 337.

29. Kambe, N.; Dresselhaus, M. S.; Dresselhaus, G.; Basu, S.; McGhie, A. R.; Fischer, J. E., Intercalate ordering in first stage graphite-lithium, Materials Science and Engineering 1979, 40, 1 .

30. Dahn, J. R., The Phase Diagram of $\mathrm{Li}_{\mathrm{x}} \mathrm{C}_{6}$, Physical Review B 1991, 44, 9170.

31. Ohzuku, T.; Iwakoshi, Y.; Sawai, K., Formation of Lithium-Graphite Intercalation Compounds in Nonaqueous Electrolytes and Their Application as a Negative Electrode for a Lithium Ion (Shuttlecock) Cell, Journal of The Electrochemical Society 1993, 140, 2490.

32. Frackowiak, E.; Gautier, S.; Gaucher, H.; Bonnamy, S.; Beguin, F., Electrochemical storage of lithium multiwalled carbon nanotubes, Carbon 1999, 37, 61 .

33. Yang, Z. H.; Wu, H. Q., Electrochemical intercalation of lithium into raw carbon nanotubes, Materials Chemistry and Physics, 2001, 71, 7.

34. Che, G. L.; Lakshmi, B. B.; Fisher, E. R.; Martin, C. R., Carbon nanotubule membranes for electrochemical energy storage and production, Nature, 1998, 393, 346.

35. Yang, Z. H.; Wu, H. Q., Electrochemical intercalation of lithium into carbon nanotubes. Solid State Ionics 2001, 143, 173.

36. Yang, Z.; Feng, Y.; Li, Z.; Sang, S.; Zhou, Y.; Zeng, L., An investigation of lithium intercalation into the carbon nanotubes by a.c. impedance, Journal of Electroanalytical Chemistry 2005, 580, 340. 
37. Claye, A. S.; Fischer, J. E.; Huffman, C. B.; Rinzler, A. G.; Smalley, R. E., SolidState Electrochemistry of the Li Single Wall Carbon Nanotube System, Journal of The Electrochemical Society 2000, 147, 2845.

38. Wang, X. X.; Wang, J. N.; Chang, H.; Zhang, Y. F., Preparation of Short Carbon Nanotubes and Application as an Electrode Material in Li-Ion Batteries, Advanced Functional Materials 2007, 17, 3613.

39. Claye, A. S.; Fischer, J. E.; Huffman, C. B.; A. G. Rinzler, A. G.; Smalley, R. E., Solid-State Electrochemistry of the Li Single Wall Carbon Nanotube System. Journal of The Electrochemical Society 2000, 147, 2845.

40. Mukhopadhyay, I.; Kawasaki, S.; Okino, F.; Govindaraj, A.; Rao, C. N. R.; Touhara, H., Electrochemical Li insertion into single-walled carbon nanotubes prepared by graphite arc-discharge method, Physica B, 2002, 323, 130.

41. Ng, S. H.; Wang, J.; Guo, Z. P.; Chen, J.; Wang, G. X.; Liu, H. K., Single wall carbon nanotube paper as anode for lithium-ion battery, Electrochimica Acta, 2005, 51, 23.

42. Frackowiak, E.; Beguin, F., Electrochemical storage of energy in carbon nanotubes and nanostructured carbons Carbon, 2002, 40, 1775.

43. Gao, B.; Kleinhammes, A.; Tang, X. P.; Bower, C.; Fleming, L.; Wu, Y.; Zhou, O., Electrochemical intercalation of single-walled carbon nanotubes with lithium, Chemical Physics Letters 1999, 307, 153.

44. Shimoda, H.; Gao, B.; Tang, X. P.; Kleinhammes, A.; Fleming, L.; Wu, Y.; Zhou, O., Lithium Intercalation into Opened Single-Wall Carbon Nanotubes: Storage Capacity and Electronic Properties, Physics Review Letters 2002, 88, 015502. 
45. Gao, B.; Bower, C.; Lorentze, J.; Fleming, L.; A. Kleinhammes, A.; Tang, X. P.; McNeil, L. E.; Wu, Y.; Zhou, O., Enhanced saturation lithium composition in ballmilled single-walled carbon nanotubes. Chemical Physics Letters 2000, 327, 69.

46. Sakamoto, J. S.; Dunn, B., Vanadium Oxide-Carbon Nanotube Composite Electrodes for Use in Secondary Lithium Batteries. Journal of The Electrochemical Society 2002, 149, A26.

47. Speck, J. S.; Endo, M.; Dresselhaus, M. S., Structure and intercalation of thin benzene derived carbon fibers. Journal of Crystal Growth 1989, 94, 834.

48. Tibbetts, G. G., Carbon fibers produced by pyrolysis of natural gas in stainless steel tubes. Applied Physics Letters 1983, 42, 666.

49. Koyama, T.; Endo, M., Electrical resistivity of carbon fibers prepared from benzene. Japanese Journal of Applied Physics 1974, 3, 1175.

50. Koyama, T.; Endo, M., Structure and properties of graphitized carbon fiber. Japanese Journal of Applied Physics 1974, 13, 1933.

51. Endo, M.; Kim, Y. A.; Hayashi, T.; Nishimura, K.; Matusita, T.; Miyashita, K.; Dresselhaus, M. S., Vapor-grown carbon fibers (VGCFs) Basic properties and their battery applications. Carbon 2001, 39, 1287.

52. Endo M.; Kim, C.; Nishimura, K.; Fujino, T.; Miyashita, K., Recent development of carbon materials for Li ion batteries. Carbon, 2000, 38, 183.

53. Kim, C.; Yang, K. S.; Kojima, M.; Yoshida, K.; Kim, Y. J.; Kim, Y. A.; Endo, M., Fabrication of Electrospinning-Derived Carbon Nanofiber Webs for the Anode Material of Lithium-Ion Secondary Batteries. Advanced Functional Materials 2006, $16,2393$. 
54. Li, C.; Yin, X.; Chen, L.; Li, Q.; Wang, T., Porous Carbon Nanofibers Derived from Conducting Polymer: Synthesis and Application in Lithium-Ion Batteries with HighRate Capability Journal of Physical Chemistry C 2009, 113, 13438.

55. Adelhelm, P.; Hu, Y. -S.; Antonietti, M.; Maierd, J.; Smarsly, B. M., Hollow Fecontaining carbon fibers with tubular tertiary structure: preparation and Li-storage properties. Journal of Materials Chemistry 2009, 19, 1616.

56. Subramanian, V.; Zhu, H.; Wei, B., High Rate Reversibility Anode Materials of Lithium Batteries from Vapor-Grown Carbon Nanofibers Journal of Physical Chemistry $B$ 2006, $110,7178$.

57. Yoo, E.; Kim, J.; Hosono, E.; Zhou, H.; Kudo, T.; Honma, I., Large Reversible Li Storage of Graphene Nanosheet Families for Use in Rechargeable Lithium Ion Batteries. Nano Letters 2008, 8, 2277.

58. Zou, L.; Kang, F.; Zheng, Y. -P.; Shen, W., Modified natural flake graphite with high cycle performance as anode material in lithium ion batteries. Electrochimica Acta $2009,54,3930$.

59. Wang, H.; Abe, T.; Maruyama, S.; Iriyama, Y.; Ogumi, Z.; Yoshikawa, K., Graphitized Carbon Nanobeads with an Onion Texture as a Lithium-Ion Battery Negative Electrode for High-Rate Use. Advanced Materials 2005, 17, 2857.

60. Liu, Q.; Cui, Z. -M.; Ma, Z.; Bian, S. -W.; Song, W. -G., Carbon Materials with Unusual Morphologies and Their Formation Mechanism. Journal of Physical Chemistry C 2007, 111, 12420. 
61. Wu, X. -L.; Liu, Q.; Guo, Y. -G.; Song, W. -G., Superior storage performance of carbon nanosprings as anode materials for lithium-ion batteries. Electrochemistry Communications 2009, 11, 1468.

62. Franklin, R. E., Proceedings of the Royal Society London Series A 1951, 209, 196.

63. Kinoshita, K., Carbon, Electrochemical and Physicochemical Properties. Wiley, New York, 1987.

64. Yazami, R.; Moreau, M., Negative electrode based on pre-lithiated carbonaceous material for a rechargeable electrochemical lithium generator, US Patent No. $5543021,1996$.

65. Xing, W.; Xue, J. S.; Zheng, T.; Gibaud, A.; Dahn, J. R., Hysteresis during Lithium Insertion in Hydrogen-Containing Carbons, Journal of The Electrochemical Society $1996,143,2137$.

66. Dahn, J. R.; Xing, W.; Gao, Y., The falling cards model for the structure of microporous carbons, Carbon 1997, 35, 825.

67. Renouard, T.; Gherghel, L.; Wachtler, M.; Bonino, F.; Scrosati, B.; Nuffer, R.; Mathis, C.; Müllen, K., Pyrolysis of hexa(phenyl)benzene derivatives: a molecular approach toward carbonaceous materials for Li-ion storage, Journal of Power Sources 2005, 139, 242.

68. Bonino, F.; Brutti, S.; Reale, P.; Scrosati, B.; Gherghel, L.; Wu, J.; Müllen, K., A Disordered Carbon as a Novel Anode Material in Lithium-Ion Cells. Advanced Materials 2005, 17, 743 . 
69. Isaev, I.; Salitra, G.; Soffer, A.; Cohen, Y. S.; Aurbach, D.; Fischer, J., A new approach for the preparation of anodes for Li-ion batteries based on activated hard carbon cloth with pore design. Journal of Power Sources 2003, 119-121, 28.

70. Ryoo, R.; Joo, S. H.; Jun, S., Synthesis of Highly Ordered Carbon Molecular Sieves via Template-Mediated Structural Transformation, Journal of Physical Chemistry B $1999,103,7743$.

71. Zhou, H.; Zhu, S.; Hibino, M.; Honma, I.; Ichihara, M., Lithium Storage in Ordered Mesoporous Carbon (CMK-3) with High Reversible Specific Energy Capacity and Good Cycling Performance. Advanced Materials 2003, 15, 2107.

72. Dey, A. N., Electrochemical Alloying of Lithium in Organic Electrolytes, Journal of The Electrochemical Society 1971, 118, 1547.

73. Idota, Y.; Kubota, T.; Matsufuji, A.; Maekawa, Y.; Miyasaka, T., Tin-Based Amorphous Oxide: A High-Capacity Lithium-Ion-Storage Material, Science 1997, $276,1395$.

74. Ying, Z.; Wan, Q.; Cao, H.; Song, Z. T.; Feng, S. L., Characterization of $\mathrm{SnO}_{2}$ nanowires as an anode material for Li-ion batteries. Applied Physics Letters 2005, 87, 113108.

75. Ying, Z.; Wan, Q.; Song, Z. T.; Feng, S. L., $\mathrm{SnO}_{2}$ nanowhiskers and their ethanol sensing characteristics, Nanotechnology 2004, 15, 1682.

76. Park, M.-S.; Wang, G.-X.; Kang, Y.-M.; Wexler, D.; Dou, S.-X.; Liu, H.-K., Preparation and Electrochemical Properties of $\mathrm{SnO}_{2}$ Nanowires for Application in Lithium-Ion Batteries, Angewandte Chemie International Edition 2007, 46, 750. 
77. Campbell, I. H.; Fauchet, P. M., The effects of microcrystal size and shape on the one phonon Raman spectra of crystalline semiconductors, Solid State Communications $1986,58,739$.

78. Park, M.-S.; Kang, Y.-M.; Wang, G.-X.; Dou, S.-X.; Liu, H.-K., Preparation and Electrochemical Properties of $\mathrm{SnO}_{2}$ Nanowires for Application in Lithium-Ion Batteries. Advanced Functional Materials. 2008, 18, 455.

79. Li, N.; Martin, C. R.; Scrosati, B., A High-Rate, High-Capacity, Nanostructured Tin Oxide Electrode for Lithium-Ion Battery Applications, Electrochemical and Solid State Letters 2000, 3, 316.

80. Kim, D. -W.; Hwang, I. -S.; Kwon, S. J.; Kang, H. -Y.; Park, K. -S.; Choi, Y. -J.; Choi, K. -J.; Park, J. -G., Highly Conductive Coaxial $\mathrm{SnO}_{2}-\mathrm{In}_{2} \mathrm{O}_{3}$ Heterostructured Nanowires for Li Ion Battery Electrodes. Nano Letters 2007, 7, 3041.

81. Wang, Y.; Lee, J. Y., Molten Salt Synthesis of Tin Oxide Nanorods: Morphological and Electrochemical Features. Journal of Physical Chemistry B 2004, 108, 17832.

82. Yuan, L.; Guo, Z. P.; Konstantinov, K.; Liu, H. K.; Dou, S. X., Nano-structured spherical porous $\mathrm{SnO}_{2}$ anodes for lithium-ion batteries. Journal of Power Sources 2006, 159, 345 .

83. Liang, Y.; Fan, J.; Xia, X.; Jia, Z., Synthesis and characterization of $\mathrm{SnO}_{2}$ nano-single crystals as anode materials for lithium-ion batteries. Materials Letters 2007, 43704373.

84. Lou, X. W.; Wang, Y.; Yuan, C.; Lee, J. Y.; Archer, L. A., Template-Free Synthesis of $\mathrm{SnO}_{2}$ Hollow Nanostructures with High Lithium Storage Capacity. Advanced Materials 2006, 18, 2325-2329. 
85. Chang, C. -C.; Liu, S. -J.; Wu, J. -J.; Yang, C. -H., Nano-tin oxide/Tin Particles on a Graphite Surface as an Anode Material for Lithium-Ion Batteries. Journal of Physical Chemistry C 2007, 111, 16423.

86. Wang, Y.; Lee, J. Y.; Zeng, H. C., Polycrystalline $\mathrm{SnO}_{2}$ Nanotubes Prepared via Infiltration Casting of Nanocrystallites and Their Electrochemical Application, Chemistry of Materials 2005, 17, 3899.

87. Wang, Y.; Zeng, H. C.; Lee, J. Y., Highly Reversible Lithium Storage in Porous $\mathrm{SnO}_{2}$ Nanotubes with Coaxially Grown Carbon Nanotube Overlayers. Advanced Materials 2006, 18,645 .

88. Wen, Z.; Wang, Q.; Zhang, Q.; Li, J., In Situ Growth of Mesoporous $\mathrm{SnO}_{2}$ on Multiwalled Carbon Nanotubes: A Novel Composite with Porous-Tube Structure as Anode for Lithium Batteries. Advanced Functional Materials 2007, 17, 2772.

89. Sivashanmugam, A.; Prem Kumar, T.; Renganathan, N. G.; Gopukumar, S.; Wohlfahrt-Mehrens, M.; Garche, J., Journal of Power Sources 2005, 144, 197.

90. Poizot, P.; Laruelle, S.; Grugeon, S.; Dupont, L.; Tarascon, J. M., Nano-sized transition-metal oxides as negative-electrode materials for lithium-ion batteries. Nature 2000, 407, 496.

91. Li, W. -Y.; Xu, L. -N.; Chen, J., $\mathrm{Co}_{3} \mathrm{O}_{4}$ Nanomaterials in Lithium-Ion Batteries and Gas Sensors. Advanced Functional Materials 2005, 15, 851.

92. Liu, Y.; Wang, G.; Xu, C.; Wang, W., Fabrication of $\mathrm{Co}_{3} \mathrm{O}_{4}$ nanorods by calcination of precursor powders prepared in a novel inverse microemulsion, Chemical Communications 2002, 1486. 
93. Du, N.; Zhang, H.; Chen, B.; Wu, J.; Ma, X.; Liu, Z.; Zhang, Y.; Yang, D.; Huang, X.; Tu, J., Porous $\mathrm{Co}_{3} \mathrm{O}_{4}$ Nanotubes Derived From $\mathrm{Co}_{4}(\mathrm{CO})_{12}$ Clusters on Carbon Nanotube Templates: A Highly Efficient Material For Li-Battery Applications. Advanced Materials 2007, 19, 4505.

94. Nam, K. T.; Kim, D. -W.; Yoo, P. J.; Chiang, C. -Y.; Meethong, N.; Hammond, P. T.; Chiang, Y. -T.; Belcher, A. M., Virus-Enabled Synthesis and Assembly of Nanowires for Lithium Ion Battery Electrodes. Nature 2006, 312, 885.

95. Li, Y.; Tan, B.; Wu, Y. J., Freestanding Mesoporous Quasi-Single-Crystalline $\mathrm{Co}_{3} \mathrm{O}_{4}$ Nanowire Arrays, Journal of American Chemical Society 2006, 128, 14258.

96. Li, Y.; Tan, B.; Wu, Y. J., Mesoporous $\mathrm{Co}_{3} \mathrm{O}_{4}$ Nanowire Arrays for Lithium Ion Batteries with High Capacity and Rate Capability. Nano Letters 2008, 8, 265.

97. Li, C. C.; Yin, X. M.; Chen, L. B.; Li, Q. H.; Wang, T. H., High capacity and excellent cycling stability of branched cobalt oxide nanowires as Li-insertion materials Applied Physics Letters 2010, 97, 043501.

98. Światowska-Mrowiecka, J.; de Diesbach, S.; Maurice, V.; Zanna, S.; Klein, L.; Briand, E.; Vickridge, I.; Marcus, P., Li-Ion Intercalation in Thermal Oxide Thin Films of $\mathrm{MoO}_{3}$ as Studied by XPS, RBS, and NRA. Journal of Physical Chemistry C 2008, 112, 11050 .

99. Jung, Y. S.; Lee, S.; Ahn, D.; Dillon, A. C.; Lee, S. -H., Electrochemical reactivity of ball-milled $\mathrm{MoO}_{3-\mathrm{y}}$ as anode materials for lithium-ion batteries. Journal of Power Sources 2009, 188, 286. 
100.Leroux, F.; Goward, G. R.; Power, W. P.; Nazar, L. F., Understanding the Nature of Low-Potential Li Uptake into High Volumetric Capacity Molybdenum Oxides, Electrochemical and Solid-State Letters 1998, 1, 255.

101.Mai, L.; Hu, B.; Chen, W.; Qi, Y.; Lao, C.; Yang, R.; Dai, Y.; Wang, Z. L., Lithiated $\mathrm{MoO}_{3}$ Nanobelts with Greatly Improved Performance for Lithium Batteries. Advanced Materials 2007, 19, 3712.

102. Mariotti, D.; Lindström, H.; Bose, A. C.; Ostrikov, K., Monoclinic $\beta$-MoO3 nanosheets produced by atmospheric microplasma: application to lithium-ion batteries. Nanotechnology 2008, 19, 495302.

103. Komaba S.; Kumagai N.; Kumagai R.; Kumagai N.; Yashiro H., Molybdenum oxides synthesized by hydrothermal treatment of $\mathrm{A}_{2} \mathrm{MoO}_{4}(\mathrm{~A}=\mathrm{Li}, \mathrm{Na}, \mathrm{K})$ and electrochemical lithium intercalation into the oxides, Solid State Ionics 2002, 152$153,319$.

104. Mahan, A. H.; Parilla, P. A.; Jones, K. M.; Dillon, A. C., HWCVD Production of High Density Crystalline $\mathrm{WO}_{3}$ Nanorods, Chemical Physics Letters 2005, 413, 88.

105.Lee, S. -H.; Kim, Y. -H.; Deshpande, R.; Parilla, P. A.; Whitney, E.; Gillaspie, D. T.; Jones, K. M.; Mahan, A. H.; Zhang, S.; Dillon, A. C., Reversible Lithium-Ion Insertion in Molybdenum Oxide Nanoparticles. Advanced Materials 2008, $20,1$.

106. Chen, J.; Xu, L.; Li, W.; Gou, X., $\alpha-\mathrm{Fe}_{2} \mathrm{O}_{3}$ Nanotubes in Gas Sensor and LithiumIon Battery Applications. Advanced Materials 2005, 17, 582.

107.Reddy, M. V.; Yu, T.; Sow, C. -H.; Shen, Z. X.; Lim, C. T.; Rao, G. V. S.; Chowdari, B. V. R., $\alpha-\mathrm{Fe}_{2} \mathrm{O}_{3}$ Nanoflakes as an Anode Material for Li-Ion Batteries. Advanced Functional Materials 2007, 17, 2792. 
108. Taberna, P. L.; Mitra, S.; Poizot, P.; Simon, P.; Tarascon, J. -M., High rate capabilities $\mathrm{Fe}_{3} \mathrm{O}_{4}$-based $\mathrm{Cu}$ nano-architectured electrodes for lithium-ion battery applications. Nature Materials 2006, 5, 567.

109. Lee, D. -H.; Park, J. -G.; Choi, K. J.; Choi, H. -J.; Kim, D. -W., Preparation of Brookite-Type $\mathrm{TiO} 2 /$ Carbon Nanocomposite Electrodes for Application to Li Ion Batteries. European Journal of Inorganic Chemistry 2008, 878.

110. Armstrong, A. R.; Armstrong, G.; Canales, J.; García, R.; Bruce, P. G., Lithium-Ion Intercalation into $\mathrm{TiO}_{2}$-B Nanowires. Advanced Materials 2005, 17, 862.

111. Wang, K. X.; Wei, M. D.; Morris, M. A.; Zhou, H. S.; Holmes, J. D., Mesoporous Titania Nanotubes: Their Preparation and Application as Electrode Materials for Rechargeable Lithium Batteries, Advanced Materials 2007, 19, 3016.

112. Wang, K.; Wei, M.; Morris, M. A..; Zhou, H.; Holmes, J. D., Mesoporous Titania Nanotubes: Their Preparation and Application as Electrode Materials for Rechargeable Lithium Batteries. Advanced Materials 2007, 19, 3016.

113. Yuan, L.; Guo, Z. P.; Konstantinov, K.; Munroe, P.; Liu, H. K., Spherical Clusters of NiO Nanoshafts for Lithium-Ion Battery Anodes. Electrochemical and SolidState Letters 2006, 9, A524.

114. Wu, M. -S.; Chiang, P. -C. J.; Lee, J. -T.; Lin, J. --C., Synthesis of Manganese Oxide Electrodes with Interconnected Nanowire Structure as an Anode Material for Rechargeable Lithium Ion Batteries. Journal of Physical Chemistry B 2005, $109,23279$. 
115. Neiner, D.; Chiu, H. W.; Kauzlarich, S. M., Low-Temperature Solution Route to Macroscopic Amounts of Hydrogen Terminated Silicon Nanoparticles, Journal of American Chemical Society 2006, 128, 11016.

116.Ma, H.; Cheng, F.; Chen, J.; Zhao, J.; Li, C.; Tao, Z.; Liang, J., Nest-like Silicon Nanospheres for High-Capacity Lithium Storage. Advanced Materials 2007, 19, 4067.

117.Park, M. -H.; Kim, M. G.; Joo, J.; Kim, K.; Kim, J.; Ahn, S.; Cui, Y.; Cho, J., Silicon Nanotube Battery Anodes. Nano Letters 2009, 9, 3844.

118. Chan, C. K.; Peng, H.; Liu, G.; McIlwrath, K.; Zhang, X. F.; Huggins, R. A.; Cui, Y., High-performance lithium battery anodes using silicon nanowires. Nature Nanotechnology 2008, 3, 31 .

119. Cui, L. -F.; Ruffo, R.; Chan, C. K.; Peng, H.; Cui, Y., Crystalline-Amorphous Core-Shell Silicon Nanowires for High Capacity and High Current Battery Electrodes. Nano Letters 2009, 9, 491.

120. Chan, C. K.; Zhang, X. F.; Cui, Y., High Capacity Li Ion Battery Anodes Using Ge Nanowires. Nano Letters 2008, 8, 307.

121.Lee, H.; Kim, H.; Doo, S. -G.; Cho, J., Synthesis and Optimization of Nanoparticle Ge Confined in a Carbon Matrix for Lithium Battery Anode Material. Journal of The Electrochemical Society 2007, 154, A343.

122. Lee, H.; Cho, J., $\mathrm{Sn}_{78} \mathrm{Ge}_{22} @$ Carbon Core-Shell Nanowires as Fast and HighCapacity Lithium Storage Media. Nano Letters 2007, 7, 2638. 
123.Hassoun, J.; Panero, S.; Scrosati, B., Electrodeposited Ni-Sn intermetallic electrodes for advanced lithium ion batteries, Journal of Power Sources 2006, 160, 1336.

124. Hassoun, J.; Panero, S.; Simon, P.; Taberna, P. L.; Scrosati, B., High-Rate, LongLife Ni-Sn Nanostructured Electrodes for Lithium-Ion Batteries. Advanced Materials 2007, 19, 1632.

125.Zhou, S.; Liu, X.; Wang, D., Si/TiSi 2 Heteronanostructures as High-Capacity Anode Material for Li Ion Batteries. Nano Letters 2010, 10, 860.

126. Kim, H.; Han, B.; Choo, J.; Cho, J., Three-Dimensional Porous Silicon Particles for Use in High-Performance Lithium Secondary Batteries. Angewandte Chemie 2008, $47,10151$.

127. Notten, P. H. L.; Roozeboom, F.; Niessen, R. A. H.; Baggetto, L., 3-D Integrated All-Solid-State Rechargeable Batteries. Advanced Materials 2007, 19, 4564-4567.

128. Shin, H. -C.; Liu, M., Three-Dimensional Porous Copper-Tin Alloy Electrodes for Rechargeable Lithium Batteries. Advanced Functional Materials 2005, 15, 582.

129.Zheng, S. -F.; Hu, J. -S.; Zhong, L. -S.; Song, W. -G.; Wan, L. -J.; Guo, Y. -G., Introducing Dual Functional CNT Networks into CuO Nanomicrospheres toward Superior Electrode Materials for Lithium-Ion Batteries. Chemistry of Materials 2008, 20, 3617 .

130. Ortiz, G. F.; Hanzu, I.; Lavela, P.; Knauth, P.; Tirado, L. J.; Djenizian, T., Nanoarchitectured $\mathrm{TiO}_{2} / \mathrm{SnO}$ : A Future Negative Electrode for High Power Density Li-Ion Microbatteries, Chemistry of Materials 2010, 22, 1926. 
131.Wang, Q.; Wen, Z.; Li, J., A Hybrid Supercapacitor Fabricated with a Carbon Nanotube Cathode and a $\mathrm{TiO}_{2}-\mathrm{B}$ Nanowire Anode. Advanced Functional Materials 2006, 16, 2141.

132. Yu, Y.; Chen, C. -H.; Shi, Y., A Tin-Based Amorphous Oxide Composite with a Porous, Spherical, Multideck-Cage Morphology as a Highly Reversible Anode Material for Lithium-Ion Batteries. Advanced Materials 2007, 19, 993.

133. Bard, A. J.; Faulkner, L. R., Electrochemical Methods: Fundamentals and Applications. Wiley, New York, 2001.

134. Li, N.; Martin, C. R., A High-Rate, High-Capacity, Nanostructured Sn-Based Anode Prepared Using Sol-Gel Template Synthesis, Journal of The Electrochemical Society 2001, 148, A164.

135. Beaulieu, L. Y.; Beattie, S. D.; Hatchard, T. D.; Dahn, J. R., The Electrochemical Reaction of Lithium with Tin Studied By In Situ AFM, Journal of The Electrochemical Society 2003,150, A419.

136. Beaulieu, L. Y.; Hatchard, T. D.; Bonakdarpour, A.; Fleischauer, M. D.; Dahn, J. R., Reaction of Li with Alloy Thin Films Studied by In Situ AFM, Journal of The Electrochemical Society 2003,150, A1457.

137. Courtney, I. A.; Tse, J. S.; Mao, O.; Hafner, J; Dahn, J. R., Ab initio calculation of the lithium-tin voltage profile, Physical Review B 1998, 58, 15583.

138. Meduri, P.; Pendyala, C.; Kumar, V.; Sumanasekera, G. U.; Sunkara, M. K., Hybrid tin oxide nanowires as stable and high capacity anodes for Li ion batteries, Nano Letters 2009, 9, 612. 
139. Winter, M.; Besenhard, J. O., Electrochemical lithiation of tin and tin-based intermetallics and composites, Electrochimica Acta 1999, 45, 31.

140. Morimoto, H.; Nakai, M.; Tatsumisago, M.; Minami, T., Mechanochemical Synthesis and Anode Properties of SnO-Based Amorphous Materials, Journal of The Electrochemical Soceity 1999, 146, 3970.

141. Li, N.; Martin, C. R., A High-Rate, High-Capacity, Nanostructured Sn-Based Anode Prepared Using Sol-Gel Template Synthesis, Journal of The Electrochemical Society 2001, 148, A164.

142. Sandu, I.; Brousse, T.; Schleich, D. M.; Danot, M., The Chemical Changes Occurring Upon Cycling of a $\mathrm{SnO}_{2}$ Negative Electrode for Lithium Ion Cell: In situ Moessbauer Investigation, Journal of Solid State Chemistry 2006, 179, 476.

143. Courtney, I. A.; Dunlap, R. A.; Dahn, J. R., In-situ ${ }^{119}$ Sn Mossbauer effect studies of the reaction of lithium with $\mathrm{SnO}$ and $\mathrm{SnO}: 0.25 \quad \mathrm{~B}_{2} \mathrm{O}_{3}: 0.25 \quad \mathrm{P}_{2} \mathrm{O}_{5}$ glass, Electrochimica Acta 1999, 45, 51.

144.Carslaw, H. S.; Jaeger, J. C. Conduction of Heat in Solids. Clarendon Press, Oxford, 1959.

145. Deshpande, R.; Qi, Y.; Cheng, Y. -T., Effects of Concentration-Dependent Elastic Modulus on Diffusion-Induced Stresses for Battery Applications. Journal of The Electrochemical Society 2010, 157, A967.

146. Cheng, Y. -T.; Verbrugge, M. W., The influence of surface mechanics on diffusion induced stresses within spherical nanoparticles, Journal of Applied Physics 2008, $104,083521$. 
147.Cheng, Y. -T.; Verbrugge, M. W., Evolution of stress within a spherical insertion electrode particle under potentiostatic and galvanostatic operation. Journal of Power Sources 2009, 190, 453.

148.Hu, J.; Bando, Y.; Xu, F.; Li, Y.; Ahan, J.; Xu, J., Growth and Field-Emission Properties of Crystalline, Thin-Walled Carbon Microtubes, Advanced Materials 2004, 16, 153 .

149. Bhimarasetti, G.; Sunkara, M. K.; Graham, U. M.; Davis, B. H.; Suh, C.; Rajan, K., Morphological Control of Tapered and Multi-junctioned Carbon Tubular Structures, Advanced Materials 2003, 15, 1629.

150. Bhimarasetti, B.; Cowley, J. M.; Sunkara, M. K., Carbon microtubes: tuning internal diameters andconical angles, Nanotechnology 2005, 16, S362.

151.Park, C.; Kim, J.; Yoon, D.; Han, S.; Doh, C.; Yeo, S.; Lee, K. H.; Anderson, T. J., Identification of a Gallium-Containing Carbon Deposit Produced by Decomposition of Trimethyl Gallium, Journal of The Electrochemical Society 2005, 152, C298.

152. Wang, Z.; Huang, X.; Xue, R.; Chen, L., A new possible mechanism of lithium insertion and extraction in low-temperature pyrolytic carbon electrode, Carbon $1999,37,685$.

153. Nemanich, R. J.; Solin, S. A., First- and second-order Raman scattering from finitesize crystals of graphite, Physical Review B 1979, 20, 392.

154. Dillon, R. O.; Woollam, J. A.; Katkanant, V., Use of Raman scattering to investigate dis-order and crystallite formation in as-deposited and annealed carbon films, Physical Review B 1984, 29, 3482. 
155. Welham, N. J.; Williams, J. S., Extended milling of graphite and activated carbon, Carbon 1998, 36, 1309.

156.Zheng, T.; Mckinnon, W. R.; Dahn, J. R., Hysteresis during Lithium Insertion in Hydrogen-Containing Carbons, Journal of The Electrochemical Society 1996, 143, 2137.

157. Shimoda, H.; Gao, B.; Tang, X. P.; Kleinhammes, A.; Fleming, L.; Wu, Y.; Zhou, O., Lithium intercalation into etched single-wall carbon nanotubes, Physica $B$ $2002,323,133$.

158. Shimoda, H.; Gao, B.; Tang, X. P.; Kleinhammes, A.; Fleming, L.; Wu, Y.; Zhou, O., Lithium intercalation into opened single-wall carbon nanotubes: storage capacity and electronic properties, Physical Review Letters 2002, 88, 015502, 1.

159. Disma, F.; Aymard, L.; Dupont, L.; Tarascon, J. M., Effect of Mechanical Grinding on the Lithium Intercalation Process in Graphites and Soft Carbons, Journal of The Electrochemical Society 1996, 143, 3959.

160. Meduri, P.; Kim, J. H.; Benjamin, H. R.; Jasinksi, J.; Sumanasekera, G. U.; Sunkara, M. K., Thin-Walled Carbon Microtubes as High-Capacity and High-Rate Anodes in Lithium-Ion Batteries. Journal of Physical Chemistry C 2010, 114, 10621.

161.Hu, Y. S.; Adelhelm, P.; Smarsly, B. M.; Hore, S.; Antonietti, M.; Maier, J., Synthesis of hierarchically porous carbon monoliths with highly ordered microstru cture and their application in rechargeable lithium batteries with high-rate capability, Advanced Functional Materials 2007, 17, 1873. 
162. Matthews, M. J.; Pimenta, M. A.; Dresselhaus, G.; Dresselhaus, M. S.; Endo, M., Origin of dispersive effects of the Raman D band in carbon materials, Physical Review B 1999, 59, R6585.

163. Prasad, B. L. V.; Sato, H.; Enoki, T.; Hishiyama, Y.; Kaburagi, Y.; Rao, A. M.; Sumanasekera, G. U.; Eklund, P. C., Intercalated nanographite: structure and electronic prop- erties, Physical Review B 2001, 64, 235407, 1.

164.Zheng, T.; Liu, Y.; Fuller, E. W.; Tseng, S.; Sacker, U. V.; Dahn, J. R., Lithium Insertion in High Capacity Carbonaceous Materials, Journal of The Electrochemical Society 1995, 142, 2581.

165. Funabiki, A.; Inaba, M.; Ogumi, Z.; Yuasa, S.; Otsuji, J.; Tasaka, A., Impedance Study on the Electrochemical Lithium Intercalation into Natural Graphite Powder, Journal of The Electrochemical Society 1998, 145, 172.

166.Zheng, T.; Xing, W.; Dahn, J. R., Carbons prepared from coals for anodes of lithium-ion cells, Carbon 1996, 34, 1501.

167. Gao, B.; Bower, C.; Lorentzen, J. D.; Fleming, L.; Kleinhammes, A.; Tang, X. P.; McNeil, L. E.; Wu, Y.; Zhou, O., Enhanced saturation lithium composition in ballmilled single-walled carbon nanotubes, Chemical Physics Letters 2000, 327, 69.

168. Dieterle, M.; Mestl, G., Raman spectroscopy of molybdenum oxides. Physical Chemistry Chemical Physics 2002, 4, 822.

169. Olson, K. A.; Schrader, G. L., in Thin Film Structures and Phase Stability, ed. Clemens B. M.; Johnson, W. L., MRS Symposium Proceedings volume 187, Materials Research Society, Pittsburgh, 1990. 
170. Cross, J. S.; Schrader, G. L., Low pressure chemical vapor deposition of molybdenum oxides from molybdenum hexacarbonyl and oxygen, Thin Solid Films 1995, 259, 5.

171. Besenhard, J. O.; Heydecke, J.; Wudy, E.; Fritz, H. P.; Foag, W., Characteristics of molybdenum oxide and chromium oxide cathodes in primary and secondary organic electrolyte lithium batteries. Part II. Transport properties, Solid State Ionics 1983, 8, 61 .

172. Yu, A.; Kumagai, N.; Liu, Z. L.; Lee, J. Y., Preparation of sodium molybdenum oxides by a solution technique and their electrochemical performance in lithium intercalation, Solid State Ionics 1998, 106, 11.

173. Dickens, P. G.; Reynolds, G. J., Transport and equilibrium properties of some oxide insertion compounds, Solid State Ionics 1981, 5, 331.

174. Spahr, M. E.; Novák, P.; Haas, O.; Nesper, R., Electrochemical insertion of lithium, sodium, and magnesium in molybdenum (VI) oxide, Journal of Power Sources $1995,54,346$. 


\section{CURRICULUM VITAE}

NAME

ADDRESS

DOB

EDUCATION
Praveen Meduri

776 David Fairleigh Court, Apt 6, Louisville, KY - 40217

Madhira, India - August 10, 1982.

Bachelor of Engineering (Hons.), Chemical Engineering, Birla Institute of Technology and Science (BITS), Pilani, India. 1999 - 2003.

GPA: $3.75 / 4$.

Master of Science,

Chemical Engineering, The University of Akron, Akron, Ohio.

$2003-2005$.

GPA: $3.57 / 4$.

Doctor of Philosophy, Chemical Engineering, The University of Louisville, Louisville, Kentucky. $2006-2010$. GPA: $3.98 / 4$.

\section{AWARDS}

Travel grant for poster presentation on "Hybrid, nano architectured tin anodes for Li-Ion batteries" at the Annual ECS meeting 2009.

$1^{\text {st }}$ prize, poster presentation on "Tin based hybrid architectures as high capacity, stable anodes for Li-Ion batteries", Engineering Exposition, University of Louisville 2009.

$3^{\text {rd }}$ prize, poster presentation on "Nanowire Hybrid Architectures as Anodes for Lithium Ion Batteries", KY NANOMAT 2008.

$3^{\text {rd }}$ prize, poster presentation on "Hybrid architectures as high capacity anodes for Li-Ion batteries", Engineering Exposition, University of Louisville 2008.

Among the top 3 students in Chemical Engineering, BITS, Pilani, India for the year 2002, 2003.

Head of the Sponsorship department of BITS, Pilani for the academic year 2001-2002.

Best exhibit award in APOGEE, BITS, Pilani, India, March 2002. 
Secured a best presentation award for a talk on "Mathematical fallacies and their Analysis" at AIMTA conference held at Chennai, 1998.

Rank $23^{\text {rd }}$ (of over 120,000 students) in the senior secondary examinations conducted by APBIE.

Rank 1481 (of over 150,000 students) in the Engineering Agriculture Medicine Common Entrance Test 1999.

\section{PATENTS}

M. K. Sunkara, P. Meduri and G.U. Sumanasekera, "High capacity anode materials for Li Ion batteries", US Provisional Patent Application 61/141,502, December 2008.

\section{PUBLICATIONS}

P. Meduri, E. Clark, G. U. Sumanasekera and M. K. Sunkara, " $\mathrm{MoO}_{3}$ nanowire arrays as high capacity anodes for lithium ion batteries" (manuscript in preparation).

P. Meduri, E. Clark, E. Dayalan, G. U. Sumanasekera and M. K. Sunkara, "Enhancement in Lithiation and de-lithiation Kinetics of Nanoscale Tin Covered Tin Oxide Nanowires Anodes for Lithium Ion Batteries" (submitted manuscript).

Praveen Meduri, Jeong H. Kim, Harry R. Benjamin, Jacek Jasinksi, Gamini U. Sumanasekera, and Mahendra K. Sunkara, "Thin-Walled Carbon Microtubes as HighCapacity and High-Rate Anodes in Lithium-Ion Batteries", J. Phys. Chem. C 114, 10621 (2010).

Praveen Meduri, Chandrashekhar Pendyala, Vivekanand Kumar, Gamini U. Sumanasekera, and Mahendra K. Sunkara "Hybrid Tin Oxide Nanowires as Stable and High Capacity Anodes for Li-ion Batteries”, Nano lett. 9(2), 612 (2009).

P. Meduri, G. U. Sumanasekera, Z. Chen, and M. K. Sunkara "Composition Controlled Synthesis and Raman Analysis of Ge-rich $\mathrm{Si}_{\mathrm{x}} \mathrm{Ge}_{1-\mathrm{x}}$ Nanowires", J. Nanosci. Nanotechno. $8,3153(2008)$.

\section{PRESENTATIONS}

Poster on "Hybrid nano architectured tin based anodes: high capacity and lithiation characteristics" at Symposium on Energy Storage Beyond Lithium Ion: Materials Perspectives, ORNL 2010.

Poster presentation on "High rate carbon materials as anodes for Li-Ion batteries" at Annual ECS meeting, Vienna, Austria, October 2009.

Poster presentation on "Nanowire based anodes for lithium ion batteries" at Ninth international annual AABC conference, Long Beach, CA, June 2009.

Poster presentation on "Hybrid architectures as high capacity anodes for Li-ion batteries" at E-expo, UofL, March 2009.

Oral presentation on "Nanowire hybrid architectures as anodes for lithium ion batteries" at Annual AIChE meeting, Philadelphia, PA, November 2008.

Poster presentation on "Nanowire hybrid architectures as anodes for lithium ion batteries" at KY NANOMAT, Louisville, KY, March 2008. 
Poster presentation on "Hybrid architectures as high capacity anodes for $\mathrm{Li}$-ion batteries" at E-expo, UofL, March 2008.

Oral presentation on "Nanowires as anode materials for Li-ion batteries" at Annual AIChE meeting, Salt Lake City, Utah, November 2007.

Oral presentation on "Nanowires as anode materials for Li-ion batteries" at Annual ECS meeting, Washington DC, October 2007.

Poster presentation on "Composition controlled synthesis of $\mathrm{Si}_{x} \mathrm{Ge}_{1-x}$ alloy nanowires" at E-expo, University of Louisville, March 2007.

Oral presentation on "Synthesis of Silicon-Germanium alloy nanowires using vaporliquid-solid method" at Annual AIChE meeting, San Francisco, CA, November 2006.

Oral presentation on" Electrospun Polyaniline/Nylon-6 and Polyaniline/Poly (vinylidene fluoride) nanowires" at Annual AIChE meeting, Cincinnati, OH, October 2005.

Poster presentation on "Surface energy characterization of nanofibers" at the Coalescence filtration nanomaterials consortium, University of Akron, OH, September 2005.

"Reactive Distillation for simultaneous production of Ethyl and Methyl Acetate", A Professional Oriented Gathering of Educational Experience (APOGEE), BITS, Pilani, India, March 2002.

\section{BOOK CHAPTERS}

S. Dumpala, G. Bhimarasetti, S. Gubbala, P. Meduri, S. Kona, and M. K. Sunkara, "Carbon Microtubes and Conical Carbon Nanotubes", Smart Materials edited by M Schwartz, (2008).

\section{PROFESSIONAL SOCIETIES}

AIChE.

ECS.

AABC.

\section{WORK EXPERIENCE}

Tutored chemical reaction engineering and Tools for Chemical Engineering classes.

Set up the distillation column and a glass reactor for the Undergraduate student lab.

Project trainee at Vasant Chemicals and Organics Ltd., Hyderabad, India working on project "Pilot plant for improvement of yield of Dinitro stilbene disulfonic acid (DNSDA) from Para nitro toluene orthosulfonic acid (PNTSA)" in the R \& D Division for a period of 6 months.

Project trainee at Madras Cements Limited, Jaggayyapet, India working on the project "Blending Control System" for a period of 2 months.

Design Project: "A dynamic study on simultaneous production of ethyl and methyl acetate using reactive distillation" for a semester as a part of undergraduate curriculum. Modeling Project: Calculation of the parameters of a typical multi-component distillation, extraction and absorption column using cubic equations of state for a semester as a part of undergraduate curriculum. 


\section{EQUIPMENT}

Plasma Enhanced Chemical Vapor Deposition, Hot Filament Chemical Vapor Deposition, Physical Vapor Deposition, Pulsed Laser Deposition, Electrochemical Techniques including Cyclic Voltammetry, Chronoamperometry, Raman Spectroscopy, Scanning Electron Microscope, X-ray Diffractometer, Transmission Electron Microscope, Atomic Force Microscope, Goniometry. 